\title{
Study of sexually transmitted infections among urban men in Pakistan: Identifying the bridging population
}

\author{
Ali M. Mir \\ Laura Reichenbach \\ Population Council \\ Abdul Wajid \\ Population Council \\ Mumraiz Khan \\ Population Council
}

Follow this and additional works at: https://knowledgecommons.popcouncil.org/departments_sbsr-rh

Part of the Demography, Population, and Ecology Commons, Family, Life Course, and Society Commons, Gender and Sexuality Commons, International Public Health Commons, Maternal and Child Health Commons, Medicine and Health Commons, and the Women's Health Commons How does access to this work benefit you? Let us know!

\section{Recommended Citation}

Mir, Ali M., Laura Reichenbach, Abdul Wajid, and Mumraiz Khan. 2008. "Study of sexually transmitted infections among urban men in Pakistan: Identifying the bridging population." Islamabad: Population Council. 


\section{Study of Sexually Transmitted Infections} Among Urban Men in Pakistan: Identifying the Bridging Population

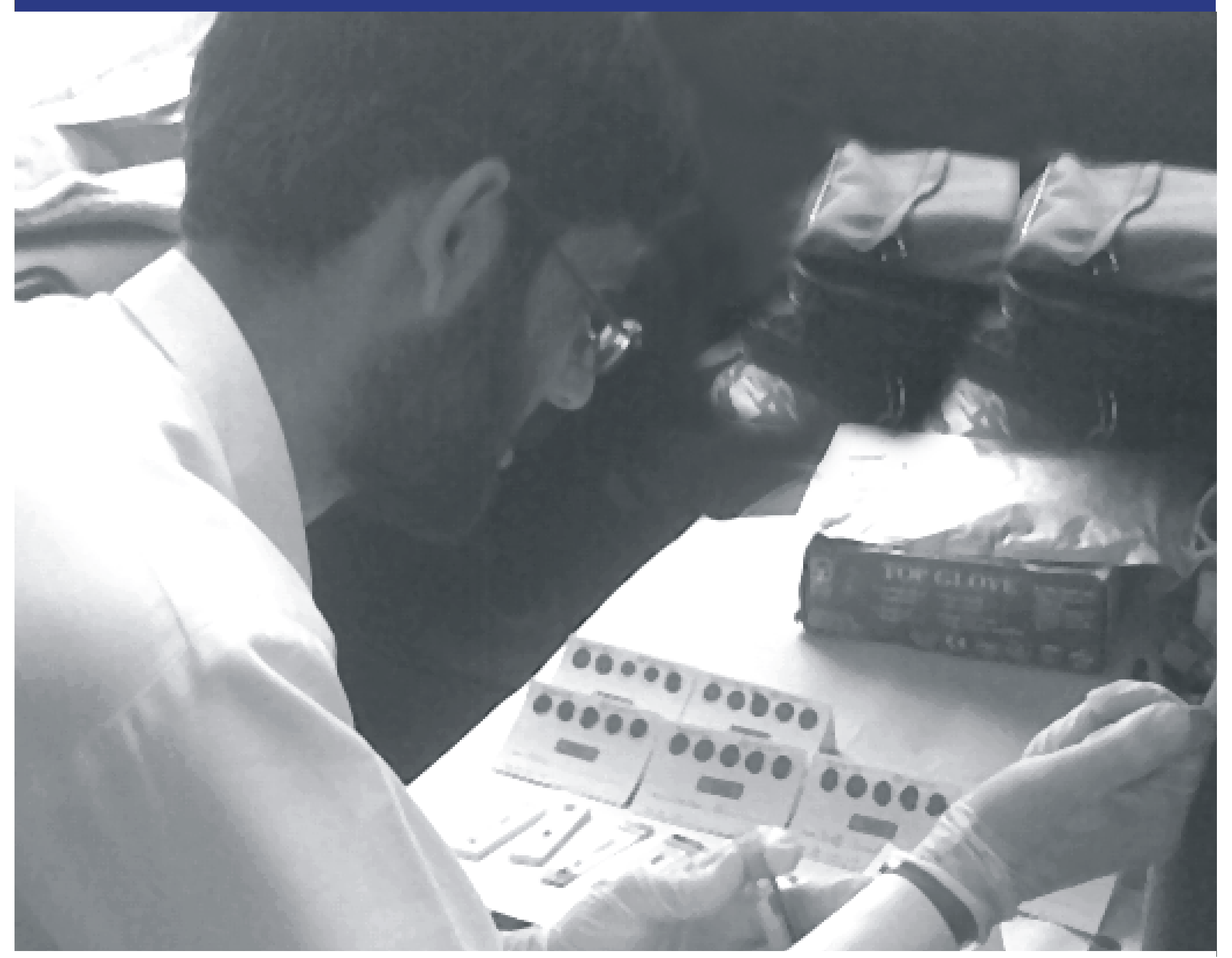
(2) Population Council

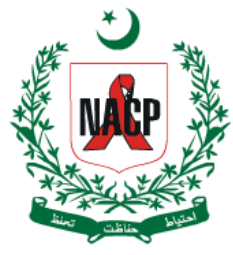





\section{Study of Sexually Transmitted Infections Among Urban Men in Pakistan: Identifying the Bridging Population}

Commissioned by

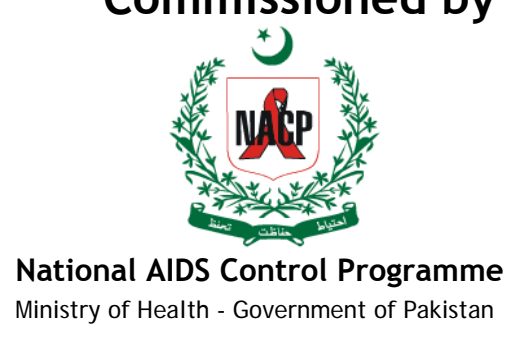

National AIDS Control Programme

Ministry of Health - Government of Pakistan

\author{
Ali M. Mir \\ Laura Reichenbach \\ Abdul Wajid \\ Mumraiz Khan
}

Conducted by

(2) Population Council

Sponsored by

DFID 


\section{(1) Population Council}

The Population Council, an international, nonprofit, nongovernmental organization established in 1952, seeks to improve the well being and reproductive health of current and future generations around the world and to help achieve a humane, equitable, and sustainable balance between people and resources.

The Council analyses population issues and trends; conducts research in the reproductive sciences; develops new contraceptives; works with public and private agencies to improve the quality and outreach of family planning and reproductive health services; helps governments design and implement effective population policies; communicates the results of research in the population field to diverse audiences; and helps strengthen professional resources in developing countries through collaborative research and programs, technical exchanges, awards, and fellowships.

For inquiries, please contact:

Population Council

House \#7, Street 62, F-6/3, Islamabad, Pakistan

Tel: 92512277439

Fax: 92512821401

Email: pcpak@popcouncil.org

Web: http://www.popcouncil.org

Layout \& Design: Ali Ammad

Published: May 2008

The Population Council reserves all rights of ownership of this document. No part of this publication may be reproduced, stored or transmitted in any form by any means electronic, photocopying, recording or otherwise without the permission of the Population Council.

\section{DIFD Disclaimer}

This document presents research funded by the UK Department for International Development (DFID) for the benefit of developing countries. The views expressed are not necessarily those of DFID. 


\section{TABLE OF CONTENTS}

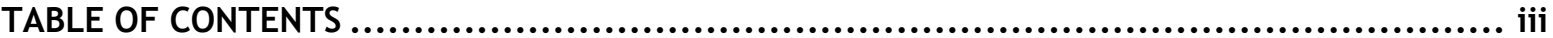

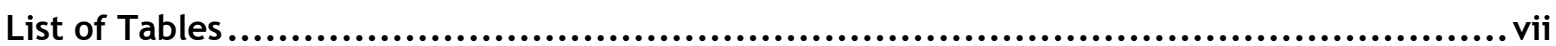

List of Figures.................................................................................

PREFACE Xiii

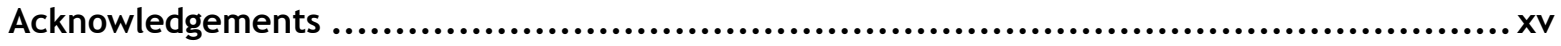

Abbreviations/Acronyms ...................................................................... xix

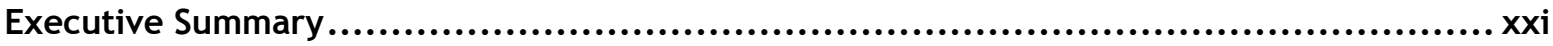

METHODOLOGY.....................................................................................

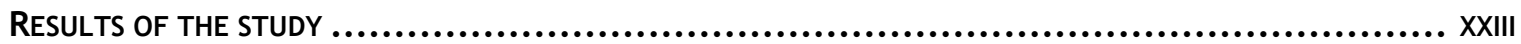

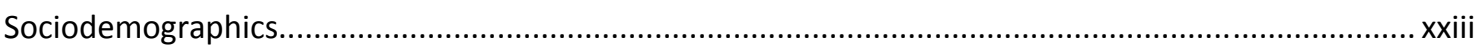

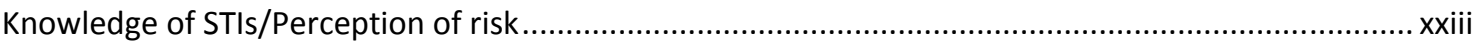

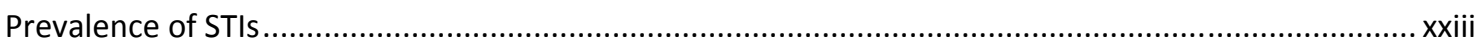

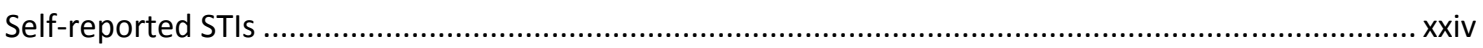

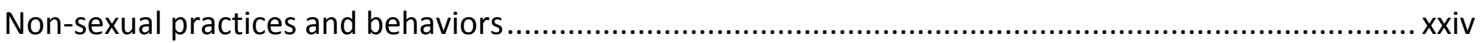

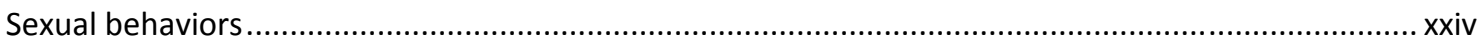

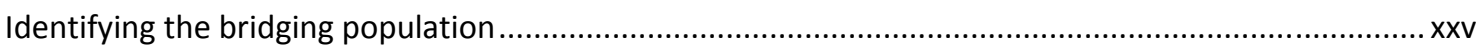

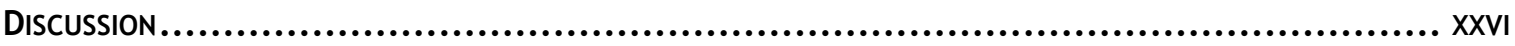

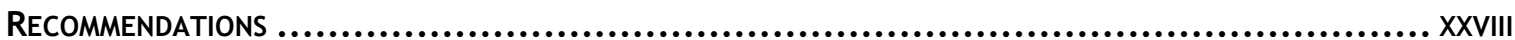

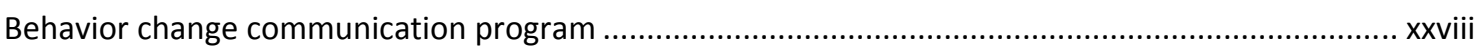

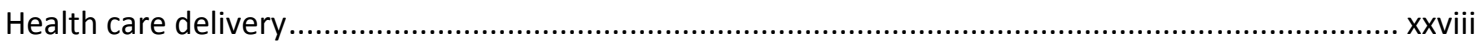

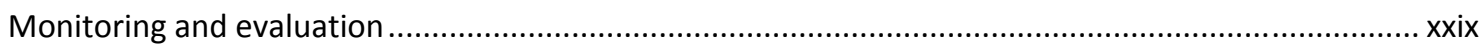

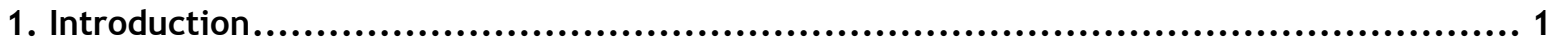

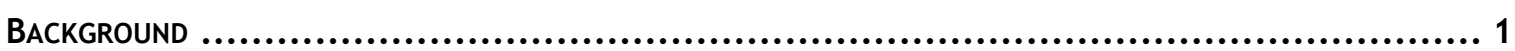

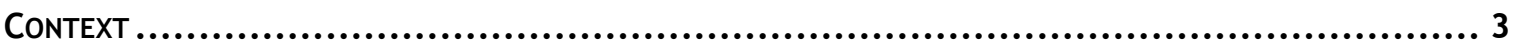

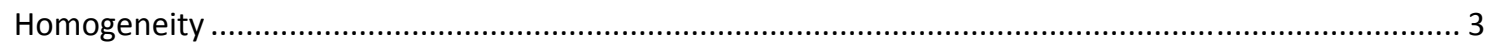

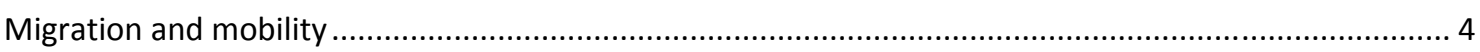

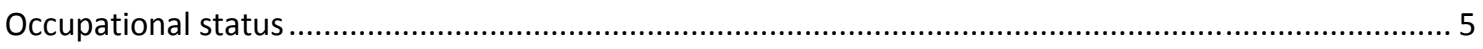

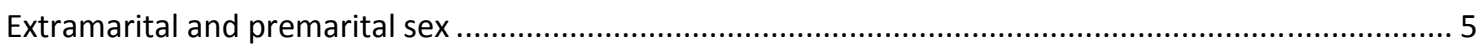

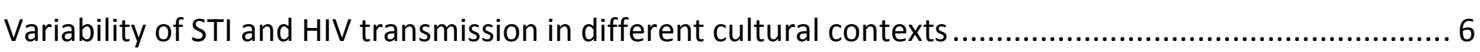

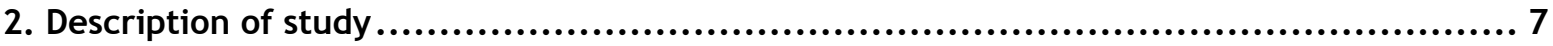

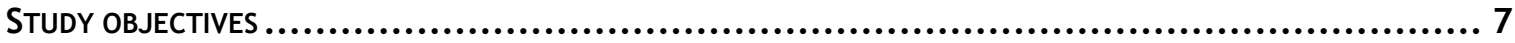

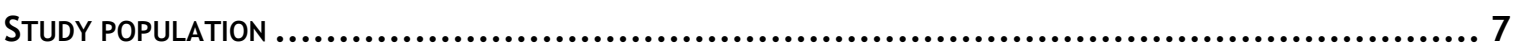

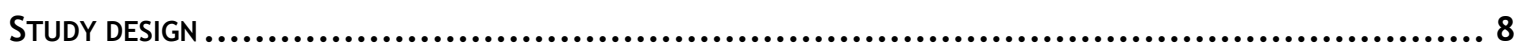

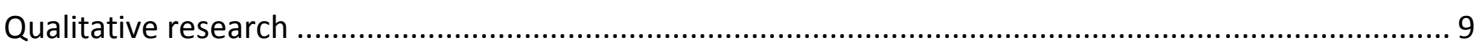

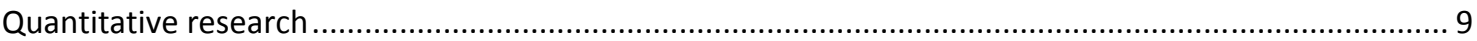

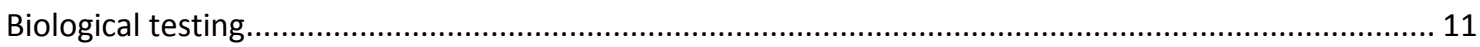




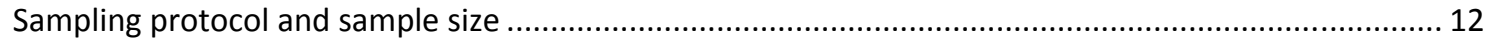

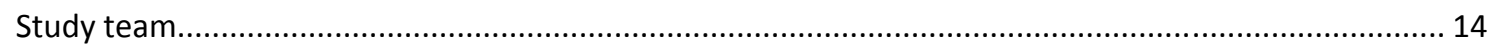

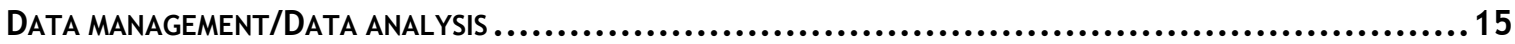

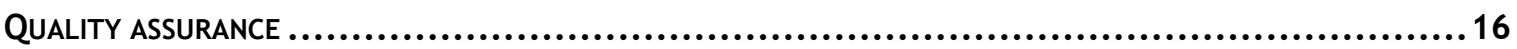

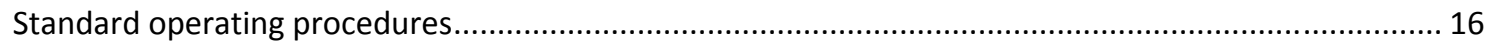

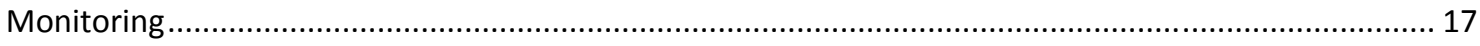

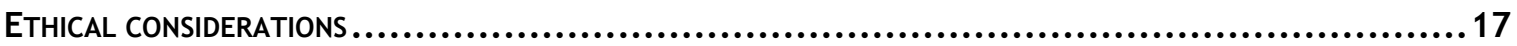

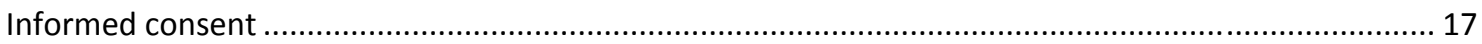

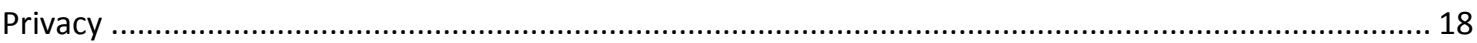

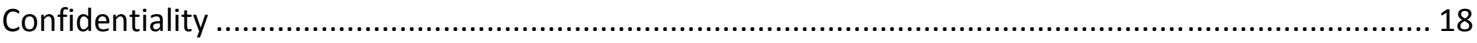

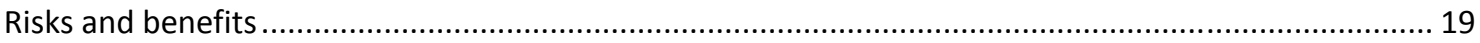

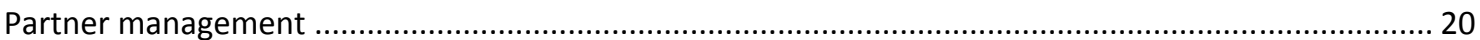

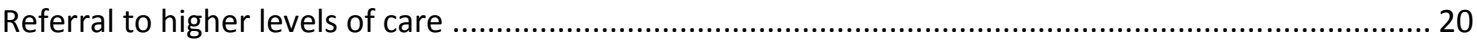

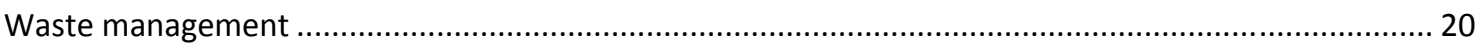

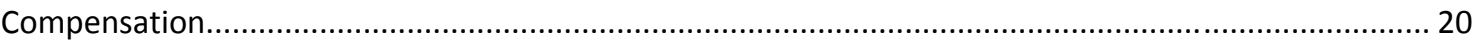

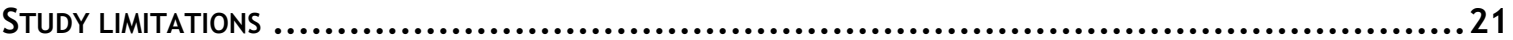

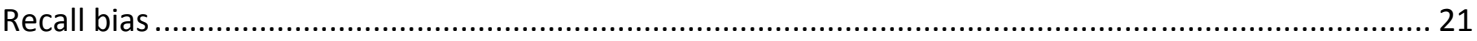

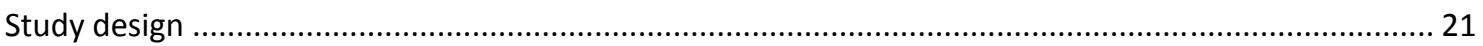

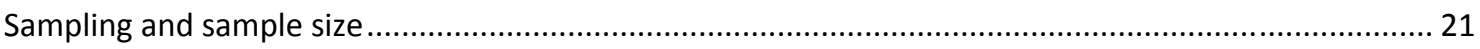

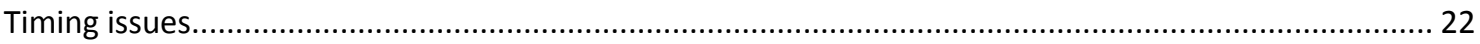

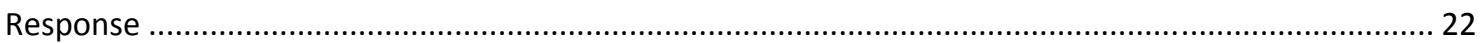

3. Sociodemographic Characteristics of respondents...........................................25

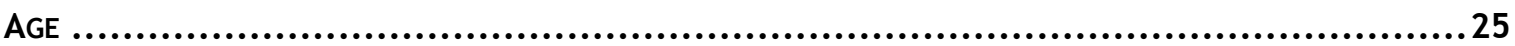

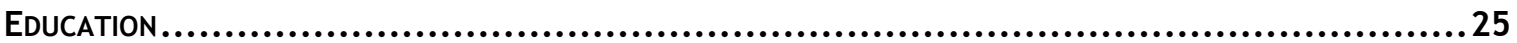

EMPLOYMENT...........................................................................................26

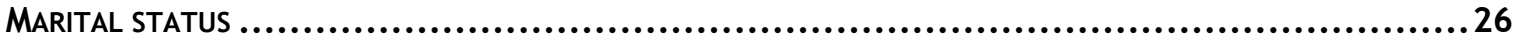

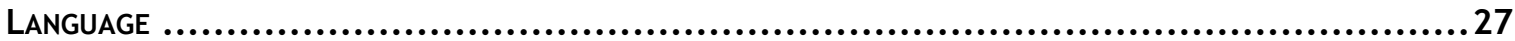

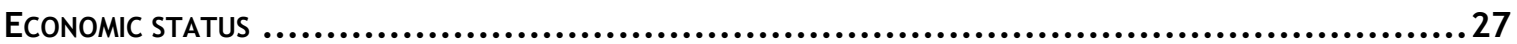

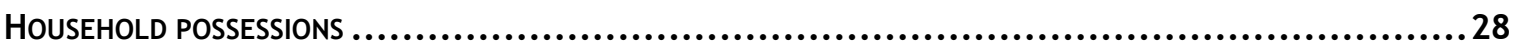

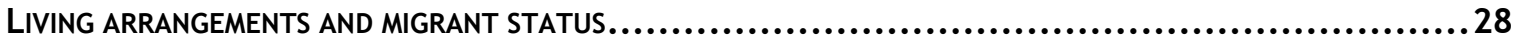

4. Awareness and Knowledge about HIVIAIDS and Other STIs ...............................31

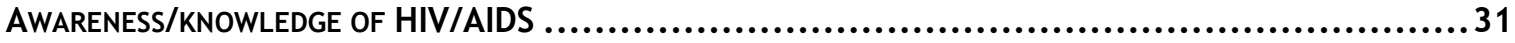

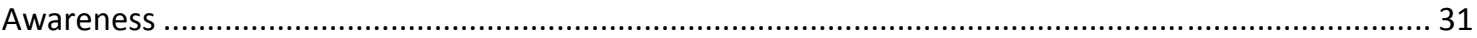

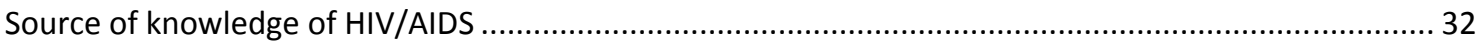

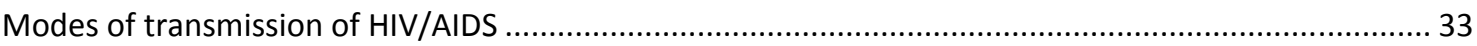

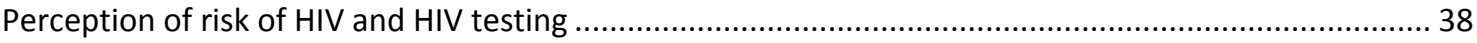

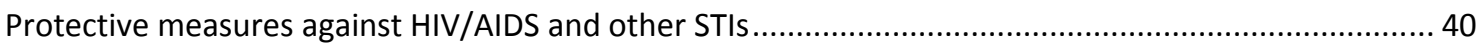

AWARENESS AND KNOWLEDGE OF OTHER STIS (INCLUDING HIVIAIDS) $\ldots \ldots \ldots \ldots \ldots \ldots \ldots \ldots \ldots \ldots \ldots \ldots \ldots \ldots$

iv 
Provider for treatment of STIs

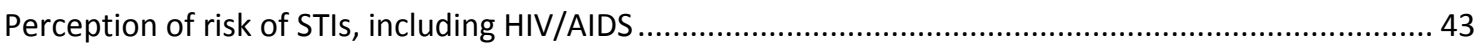

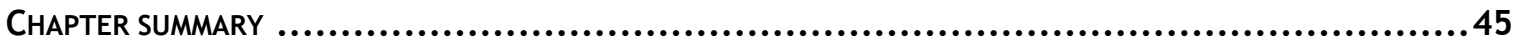

5. STI Prevalence, SIGNS, Symptoms, and TREATMENT SEEKING BEHAVIOR .....................47

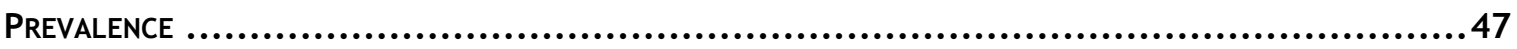

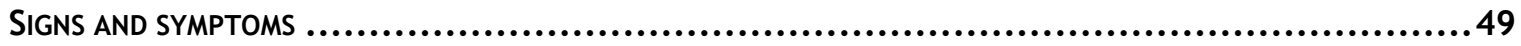

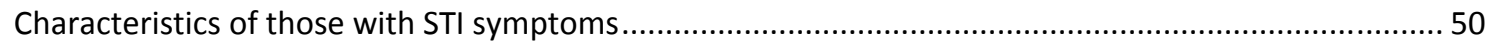

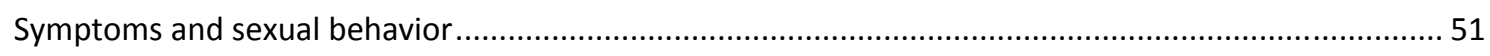

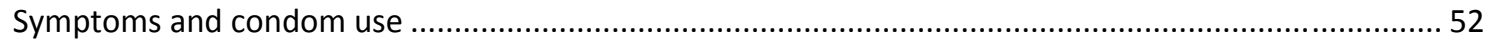

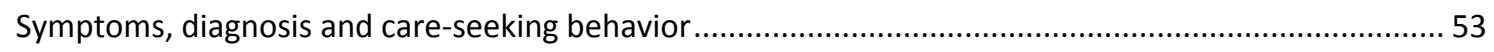

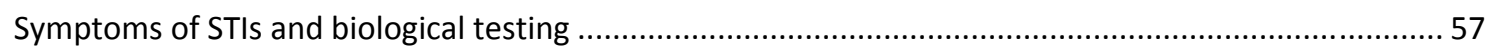

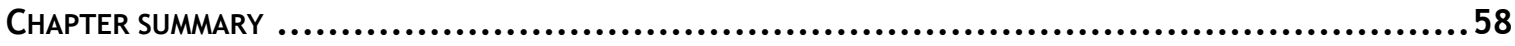

6. Sexual and NonSexual Behaviors Related to STI Prevalence and Transmission................61

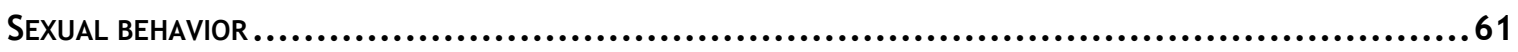

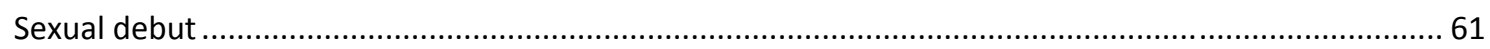

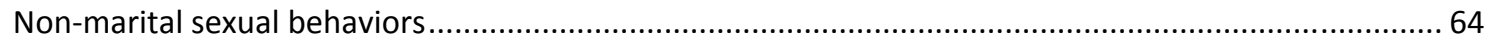

Understanding non-marital sexual behaviors: findings from the qualitative research .........................68 68

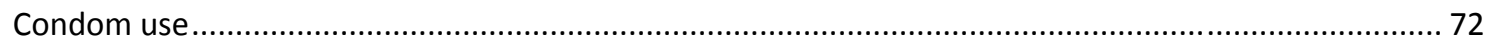

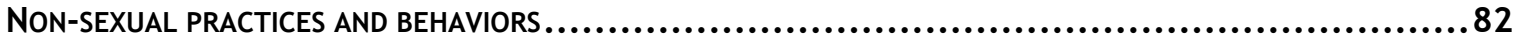

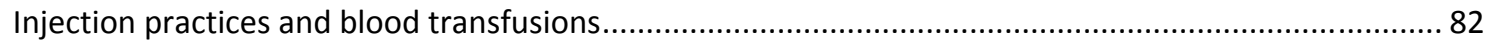

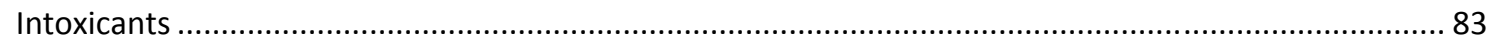

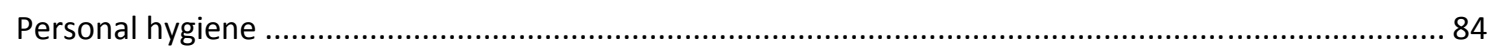

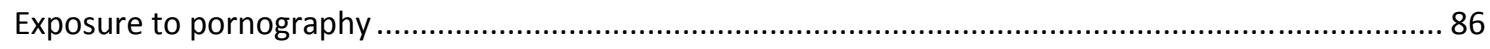

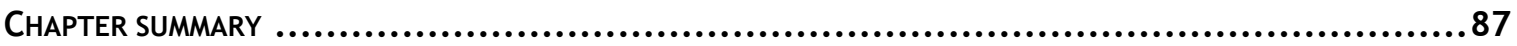

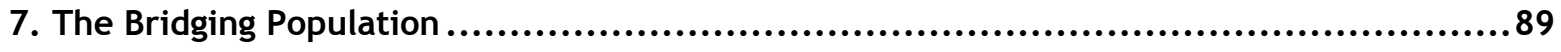

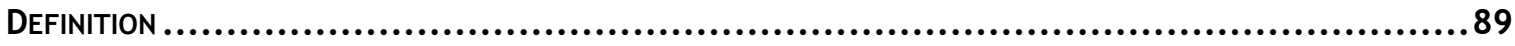

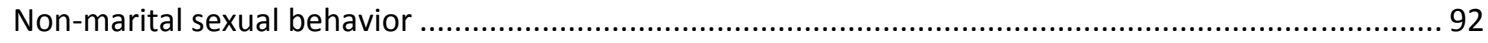

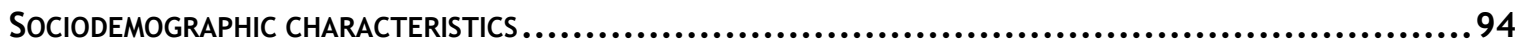

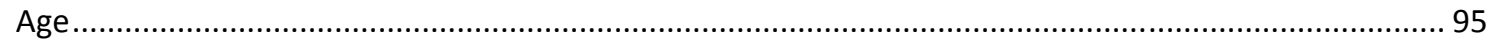

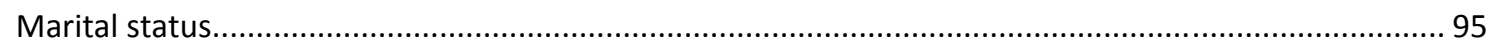

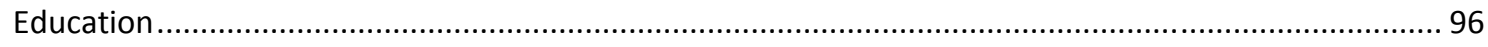

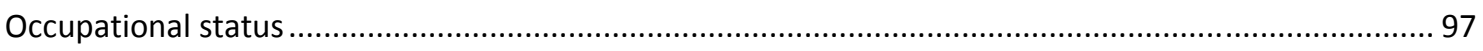

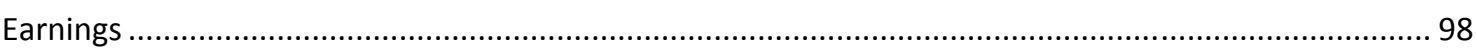

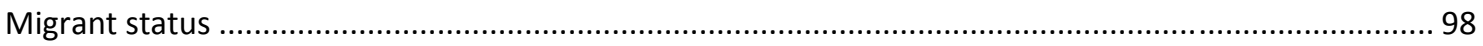

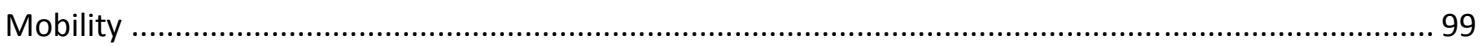

SEXUAL BEHAVIORS AND STI PREVALENCE......................................................100

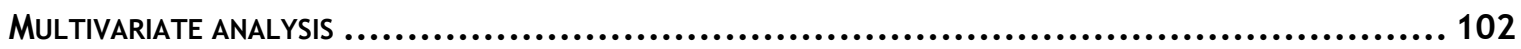

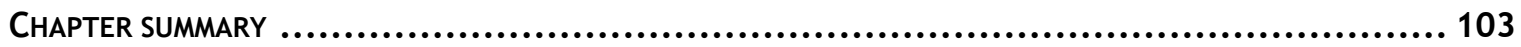

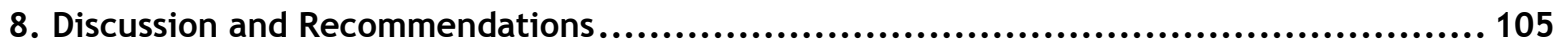




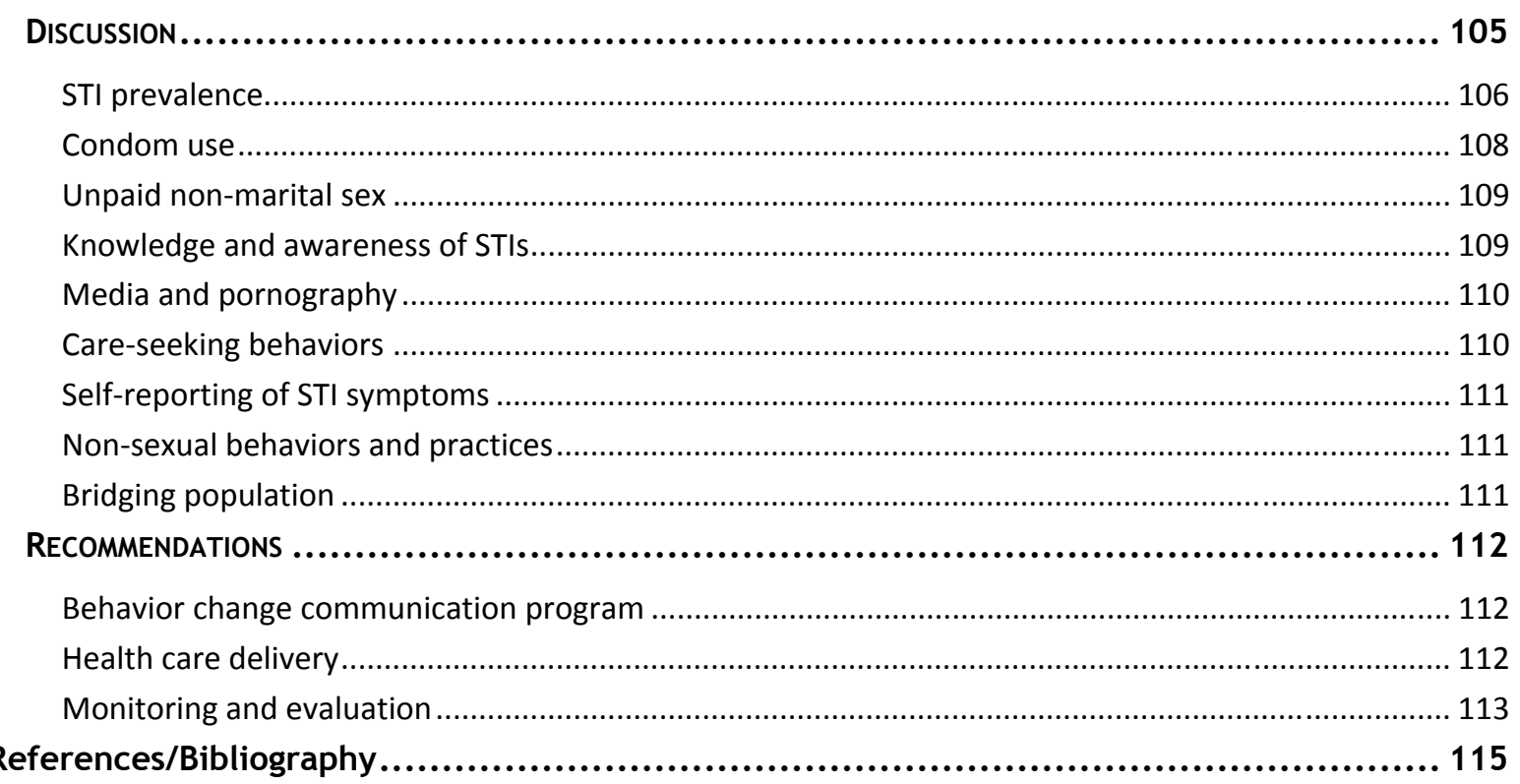

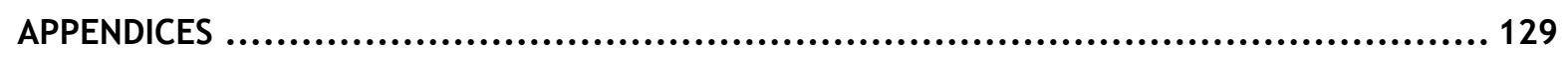

Appendix 1: City Wise Details of Respondents ............................................. 131

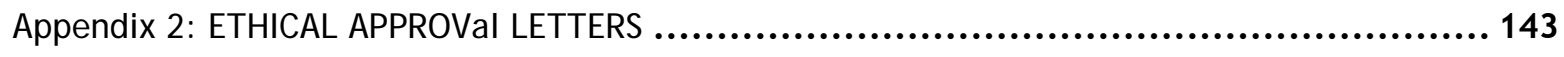

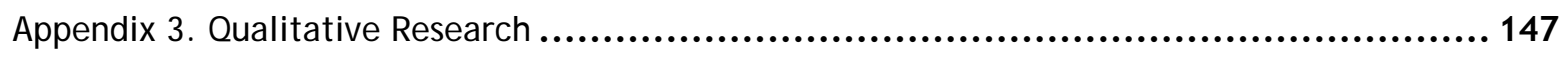

Appendix 4. Team Members .................................................................... 171

Appendix 5. Roles and Responsibilities of Each Team Member .................................. 175

Appendix 6. In-depth Interview (IDI) Guide ............................................. 179

Appendix 7. Focus Group Discussion (FGD) Guide........................................ 185

Appendix 8. Steps in Fieldwork ........................................................ 191

Appendix 9. Miscellaneous: letter and documents.......................................... 197

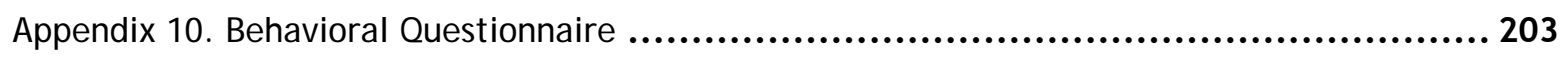




\section{LIST OF TABLES}

Table 2.1: Sequencing of research for survey of the bridging population $\ldots \ldots \ldots \ldots \ldots \ldots \ldots \ldots . \ldots$

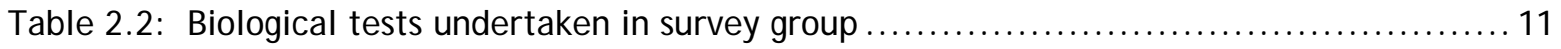

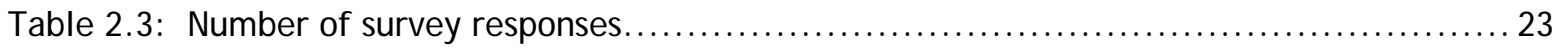

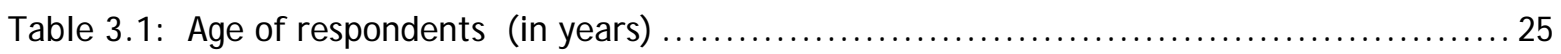

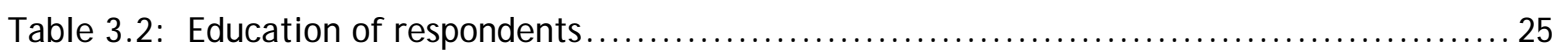

Table 3.3: Employment of respondents................................................. 26

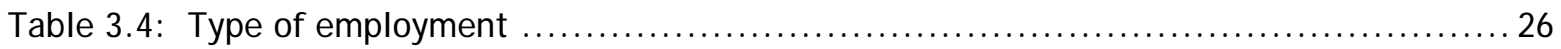

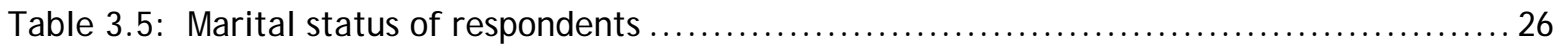

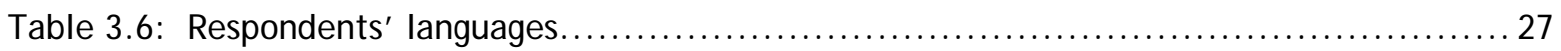

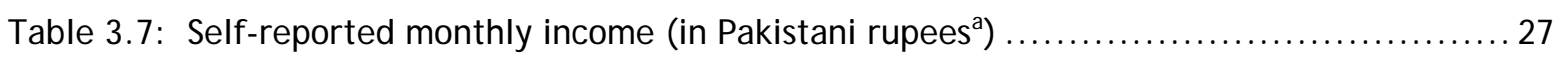

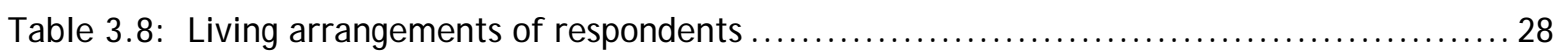

Table 4.1: Percentage distribution of respondents according to source of information regarding

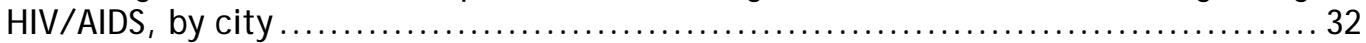

Table 4.2: Percentage distribution of respondents who had heard the term HIV/ AIDS, according to knowledge about common modes of HIV transmission, by city .................. 34

Table 4.3: Percentage distribution of respondents who had heard the term HIV/ AIDS according to knowledge about other modes of HIV/ AIDS transmission, by city ................. 35

Table 4.4: Percentage distribution of respondents who had heard of HIV/ AIDS, according to

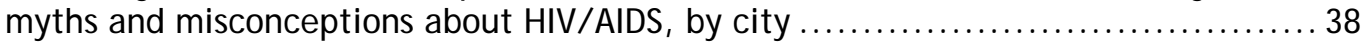

Table 4.5: Percentage distribution of men who had their blood tested for HIV/ AIDS, by city ...... 39

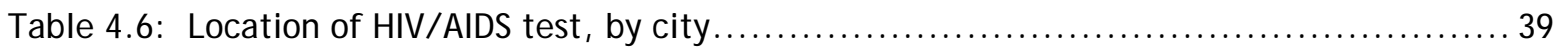

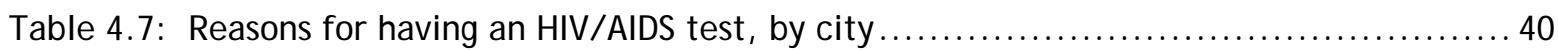

Table 4.8: Percentage distribution of respondents who knew about HIV/ AIDS, according to knowledge of modes of protection against STIs (including HIV/ AIDS), by city .

Table 4.9: Percentage distribution of unprompted knowledge of sexually transmitted infections, by city

Table 4.10: Percentage distribution of prompted knowledge of sexually transmitted infections,

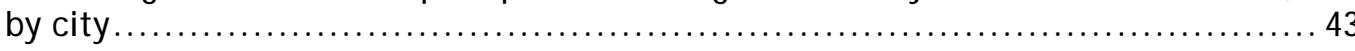

Table 4.11: Percentage distribution of men who think they are at risk of acquiring an STI, by

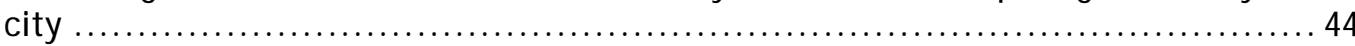

Table 4.12: Percentage distribution of men according to their ranking of the risk of acquiring

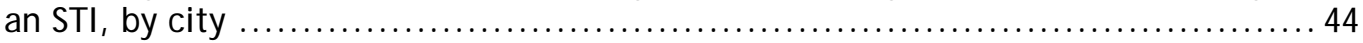

Table 4.13: Percentage of men considering themselves at risk for STIs and reporting non-

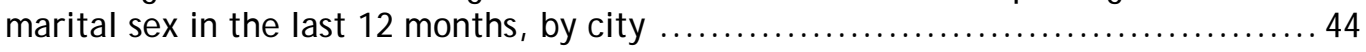

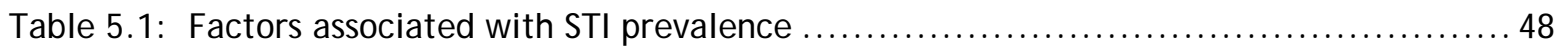


Table 5.2: Characteristics of respondents according to whether they did or did not report STI

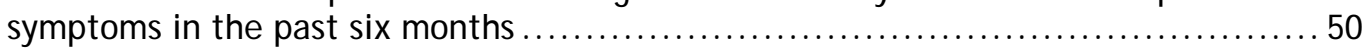

Table 5.3: Use of condom at last non-marital sexual intercourse among those reporting any STI

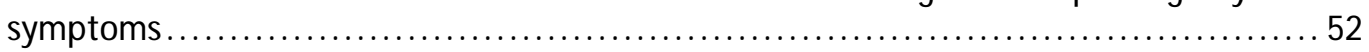

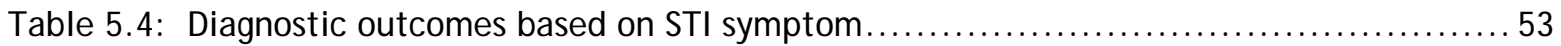

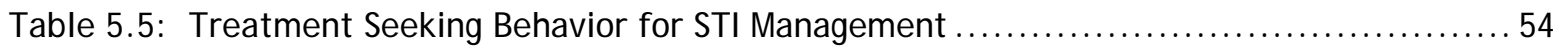

Table 5.6: Cross-tabulation of specific STI symptoms with confirmed STI on biological testing ... 57

Table 5.7: Distribution of respondents who did or did not report any STI symptoms according to laboratory findings of the presence of an STI .................................. 58

Table 6.1: Type of sexual partners of those who had non-marital sex in lifetime, by city ........ 66

Table 6.2: Number of lifetime non-marital sexual partners, by city $\ldots \ldots \ldots \ldots \ldots \ldots \ldots \ldots \ldots \ldots 7$

Table 6.3: Non-marital sexual activity over three time periods, by migrant status ( $>4$ months)

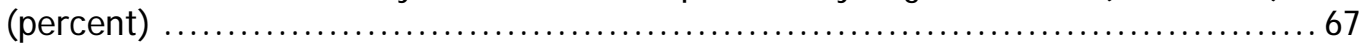

Table 6.4: Non-marital sexual activity in 3 time periods, by traveler status ( $>1$ night out of

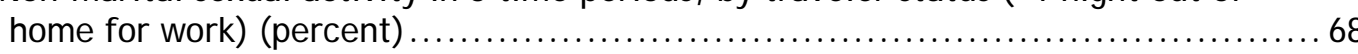

Table 6.5: Percentage distribution of respondents by reason for condom use last time and

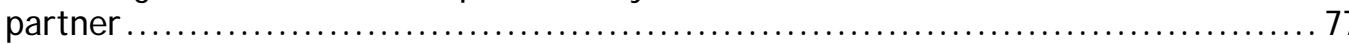

Table 6.6: Percentage distribution of respondents by reasons for not using condom last time

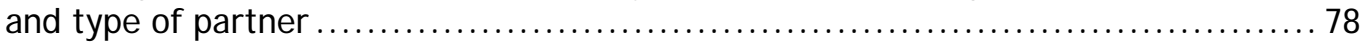

Table 6.7: Percentage of condom use with wife and extramarital partner at last sexual

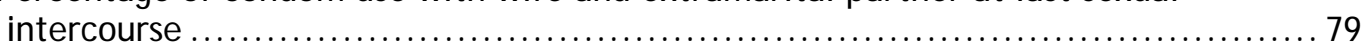

Table 6.8: Frequency of condom use in last 12 months, by type of partner .................. 80

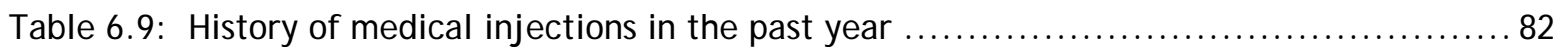

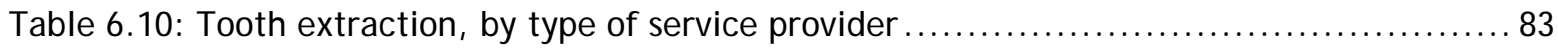

Table 6.11: Percentage distribution of respondents according to personal hygiene practices, by

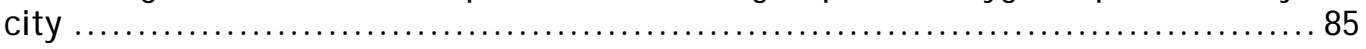

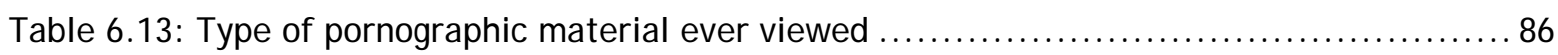

Table 6.12: Respondents who had ever viewed pornographic material $\ldots \ldots \ldots \ldots \ldots \ldots \ldots \ldots \ldots . \ldots 6$

Table 6.14: Percentage who viewed pornographic material, by marital status .................. 86

Table 6.15: Type of pornographic material ever viewed by those who ever had non-marital sex.. 86

Table 7.1: Defining the STI bridging population in Pakistan .............................. 91

Table 7.2: Type of non-marital sexual partner over last 12 months, by city ................... 93

Table 7.3: Respondents according to type of male sexual partner within the last 12 months ..... 93

Table 7.4: STI prevalence within the bridging and non-bridging populations .................. 100

Table 7.5: STI prevalence with reference to lifetime and last 12 month non-marital sexual

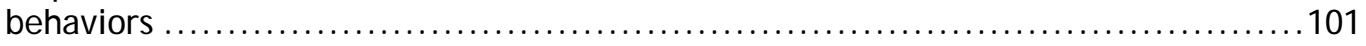

Table 7.6: Percentage of bridging and non-bridging population self-reporting STI symptoms ... 101

Table 7.7: Percentage of bridging population and non-bridging population reporting condom

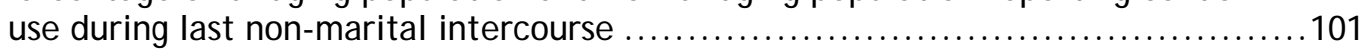


Table 7.8: Sociodemographic factors associated (unadjusted) with bridging and non-bridging

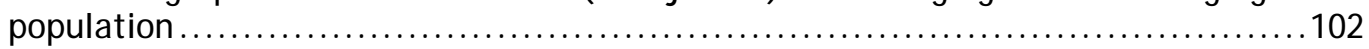

Table 7.9: Sociodemographic factors associated (adjusted) with bridging population ...........103

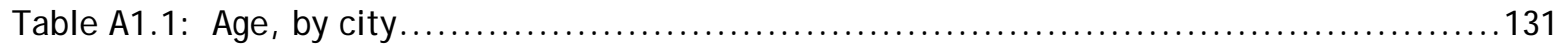

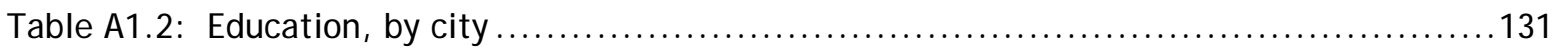

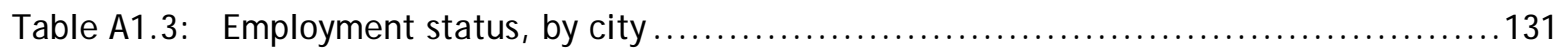

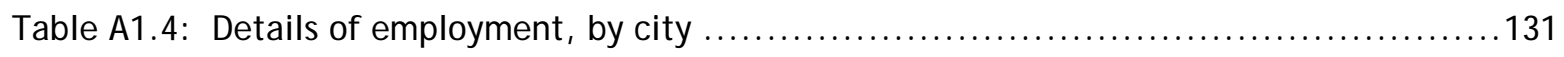

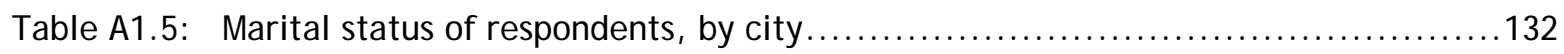

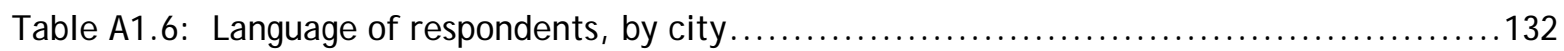

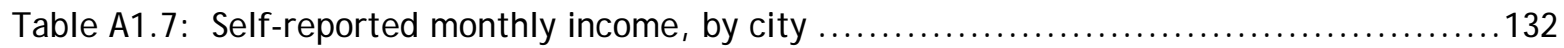

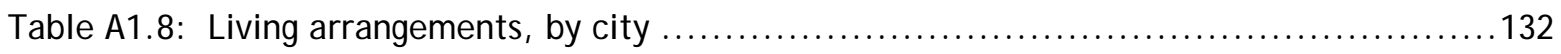

Table A1.9: Personal experience with Ulcer/Sores ................................... 133

Table A1.10: Personal experience with Purulent discharge .............................. 134

Table A1.11: Personal experience with Pain during urination ............................. 135

Table A1.12: Reason for using condom last time according to type of partner, by city $(n=130)$.. 137

Table A1.13: Consistency of condom use in last 12 months according to type of partner, by city

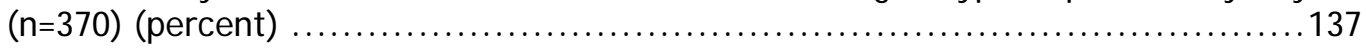

Table A1.14: STI prevalence, by city (by individual infected cases) $\ldots \ldots \ldots \ldots \ldots \ldots \ldots \ldots \ldots \ldots \ldots$

Table A1.15: Sociodemographic characteristics of bridging and non-bridging population ........139

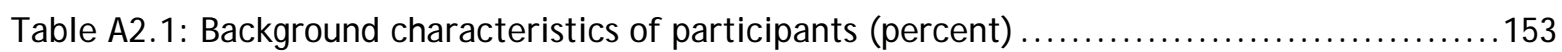




\section{LIST OF FIGURES}

Figure 3.1: Percentage of married men, within age groups............................... 27

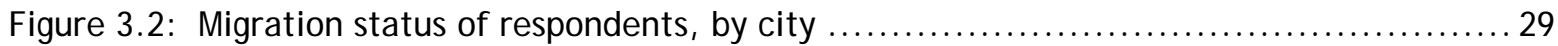

Figure 4.1: Percentage of respondents who had heard the term HIV/ AIDS $(95 \% \mathrm{Cl})$, by city ...... 32

Figure 4.2: Percentage distribution of respondents according to level of knowledge of HIV

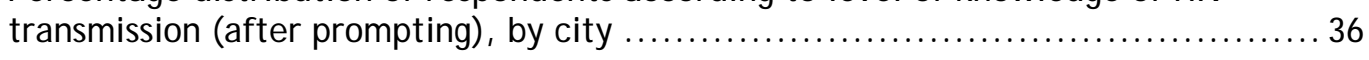

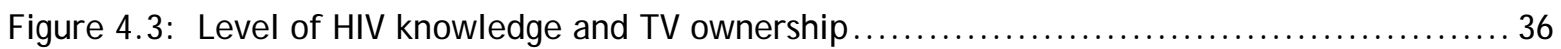

Figure 4.4: Level of HIV knowledge and socioeconomic status ............................ 37

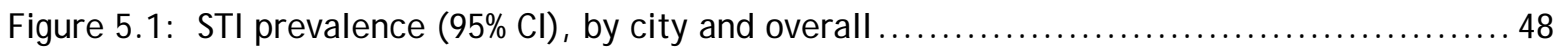

Figure 5.2: STI symptoms among those who had non-marital sex within a specified time prior

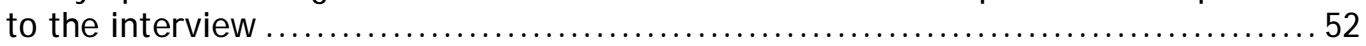

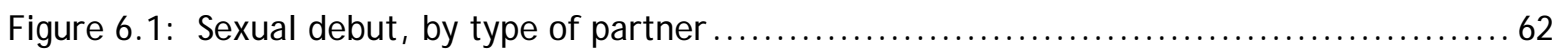

Figure 6.2: Non-marital sexual debut, by type of partner (percent) $\ldots \ldots \ldots \ldots \ldots \ldots \ldots \ldots \ldots \ldots 2$

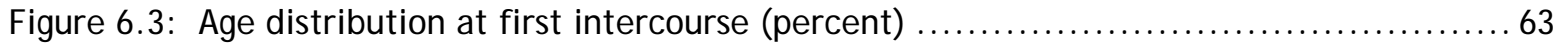

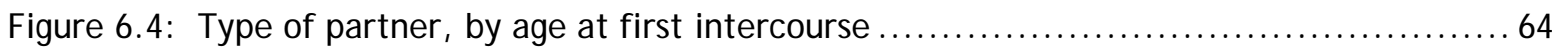

Figure 6.5: Lifetime non-marital sexual behavior of all respondents....................... 64

Figure 6.6: Respondent's non-marital sexual behavior, over three time periods (with $95 \% \mathrm{Cl}$ ) ... 65

Figure 6.7: Type of high-risk sexual partner, by marital status (percent) $\ldots \ldots \ldots \ldots \ldots \ldots \ldots \ldots 67$

Figure 6. 8: Percentage distribution of men who ever used a condom, by city $\ldots \ldots \ldots \ldots \ldots \ldots \ldots 73$

Figure 6.9: Percentage distribution of men who used a condom during their last non-marital

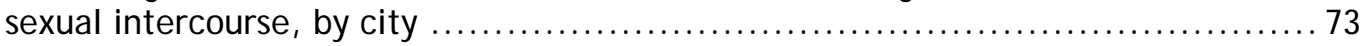

Figure 6.10: Percentage distribution of men who used condom during last non-marital

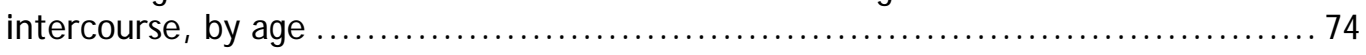

Figure 6.11: Percentage distribution of men who used a condom during last non-marital intercourse, by educational level ........................................... 75

Figure 6.12: Percentage distribution of men who used a condom during last non-marital

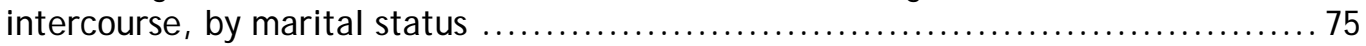

Figure 6.13: Percentage distribution of respondents according to condom use at last nonmarital sexual intercourse, by migrant status................................. 76

Figure 6.14: Frequency of condom use during the last 12 months in non-marital sexual relations. 80

Figure 6.15: Self-reported blood screening before blood transfusion $\ldots \ldots \ldots \ldots \ldots \ldots \ldots \ldots \ldots . \ldots 3$

Figure 6.16: Intoxicant use among those having non-marital sex in the last 12 months .......... 84

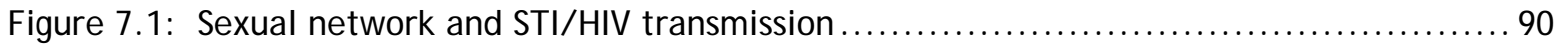

Figure 7.2: Percentage distribution, with $95 \% \mathrm{Cl}$, of respondents according to number of nonmarital partners in last 12 months, by marital status............................ 94 


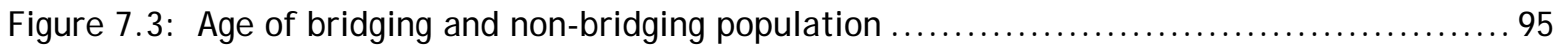

Figure 7.4: Marital status of bridging and non-bridging population ....................... 96

Figure 7.5: Educational status of bridging and non-bridging population ................... 97

Figure 7.6: Occupational status of the bridging and non-bridging population $\ldots \ldots \ldots \ldots \ldots \ldots \ldots 9 . \ldots \ldots$

Figure 7.7: Self-reported monthly earnings of bridging and non-bridging population, in

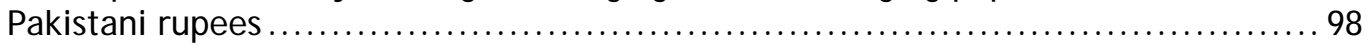

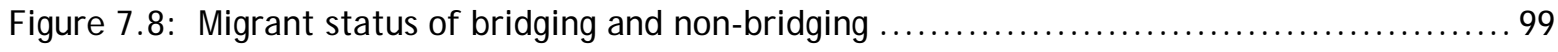

Figure 7.9: Traveler status of bridging and non-bridging population $\ldots \ldots \ldots \ldots \ldots \ldots \ldots \ldots \ldots \ldots$

Figure A1.1: Condom use during last (non-marital) sexual intercourse, by age and city ( $n=697)$. 140

Figure A1.2: Condom use during last (non-marital) sexual intercourse, by educational level and

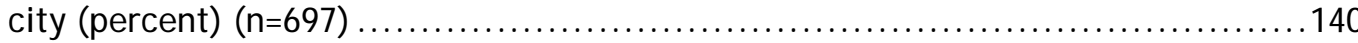

Figure A1.3: Condom use during last non-marital sexual intercourse, by marital status and city $(n=697)$.....

Figure A1.4: Condom use at last non-marital sexual intercourse, by migrant status and city (percent) $(n=697)$ 



\section{PREFACE}

Pakistan is currently classified as having a concentrated epidemic among high risk groups particularly injecting drug users (IDUs). In the recent years the country has witnessed a shift in the epidemiological trends of the disease with a particularly rapid increase in HIV prevalence being noted in the high risk groups particularly the Injecting Drug Users and now in the Male Sex Workers. The Government of Pakistan is fully cognizant of the gravity of the situation and committed to preventing the further spread of the infection from the high risk groups into the general population.

The National Survey of Sexually Transmitted Infections among Urban Men in Six major Cities of Pakistan was commission by the National AIDS Control Program and funded by the Department for International Development, UK. The Population Council has been the implementing partner for the study and has conducted the study at a very pertinent time and provided basis for understanding the risk and HIV status of Urban Men in the country. The study provides city wise prevalence of five common sexually transmitted infections as well as comprehensive information on the existing levels of knowledge regarding modes of STI transmission, condom use, sexual behaviour and risky practices. Above all the study identifies for Pakistan the characteristics of the bridging population who could act as the medium for the transmission of the infection to the low risk population. The wealth of information from the survey is extremely useful from a programmatic and policy development perspective. The study results and recommendations have appropriately highlighted the areas of vulnerability that require programmatic interventions, particularly the need to develop youth focused and gender sensitive programs that contribute to improving levels of knowledge and providing youth specific reproductive health services. The results also demonstrate the disparity across cities in Pakistan in terms of STI epidemiology, warranting city specific interventions that take into consideration the unique socio-demographic characteristics of each city

I would also like to commend and congratulate the team from the Population Council for the well coordinated activity under challenging circumstances and focal people at the National AIDS Control Program for their guidance during the planning, data collection and report development process

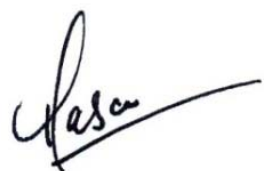

Dr. Hasan Abbas Zaheer

National Program Manager

National AIDS Control Program 



\section{ACKNOWLEDGEMENTS}

The Study of Sexually Transmitted Infections Among Urban Men in Pakistan: Identifying the Bridging Population was commissioned by the National AIDS Control Program, Government of Pakistan, with funding from the Department for International Development (DFID), United Kingdom. We thank Dr. Jane Edmondson and Dr. Raza Ali Zaidi of DFID for their support throughout this project.

We are indeed most thankful to Dr. Asma Bokhari, National Program Manager, National AIDS Control Program and her team, for their unstinted support and wholehearted cooperation during all phases of the implementation of this project. We are also grateful for the support and help of the Provincial AIDS Control Program managers in Punjab, Sindh, NWFP and Balochistan, and the Voluntary Counseling and Testing (VCT) centers in these provinces.

A large number of individuals and institutions were involved in the various stages of project implementation: it is not possible to acknowledge by name everyone who made this study possible. We are very grateful to Dr. Rana Muzaffar, Head of Molecular Biology at Sindh Institute of Urology and Transplantation (SIUT), and her team, Dr. Salma Batool and Mr. Sabz Ali, for their cooperation during the trainings and for meeting the challenge of testing a large number of biological specimens in a short period of time.

At the inception stage and during the final review of the completed report we benefited enormously from the suggestions and insights of international experts, including Dr. Fariyal Fikree, Population Reference Bureau (PRB), USA, and Dr. Shireen Jeejebhoy, Population Council, India. Dr. Johannes Van Dam, Population Council, New York, provided useful comments on the questionnaire.

We are most grateful to faculty members of the departments of community medicine of the University of Karachi, Dow University of Health Sciences, Karachi

; Rawalpindi Medical College, Rawalpindi; Institute of Public Health, Quetta; Institute of Public Health, Lahore; King Edward Medical University, Lahore; Khyber Teaching Hospital, Peshawar; and Punjab Medical College, Faisalabad, who served as study team leaders in the six study sites. We also owe special thanks to the heads of departments of sociology at the University of Karachi, Karachi; University of Punjab, Lahore; Peshawar University, Peshawar; and the Mahec Trust for Community Development, Quetta, 
Balochistan, for identifying the field researchers. Dr. Sikander Sohani, Health Advisor, Aahung, Karachi, played a key role in providing trainings to the medical team members.

We would like to express our deep appreciation to colleagues at the Population Council. Dr. Zeba Sathar, Country Director of the Islamabad office, was the overall supervisor for the study and provided invaluable guidance and support. Dr. Arshad Mehmood, Director, Research, Monitoring and Evaluation, was instrumental in resolving methodological and statistical issues as they came up. Mr. Peter Miller, Deputy Country Director, gave useful advice on issues related to sampling. Mr. Imran Ahmed, Director, Finance and Administration, and his team, which included Mr. Badr-ul-Islam and Mr. Kashif Mehmood, expertly handled all financial matters. Tayyaba Naheed was also a key member of the team. Mr. Mohammad Ashraf helped with drawing up the sample. Mr. Irfan Masood and the data collection team worked very hard and often late hours to complete the data entry and analysis on time. Computer support was made available by Mr. Muhammad Khalil, Manager, Computer Section. Mr. Mansoor Qaisar, project secretary, handled a number of logistical and secretarial assignments in a most efficient and cheerful manner, often beyond the call of duty.

During the data analysis phase we were assisted by eminent experts. Dr. Sarah Javaid helped in the analysis of the qualitative data, and Dr. Stephen Pearson, University of Leeds, United Kingdom, helped to develop the analysis plan and provided valuable feedback throughout the study. We are most grateful to Dr. Syed Abdul Mujeeb, Jinnah Postgraduate Medical Center (JPMC), Karachi, who provided valuable suggestions and key technical inputs throughout the analysis and write-up phases.

The report was finally reviewed by Dr. Naomi Rutenberg, Director, HIV and AIDS Program, Population Council, New York. We are most grateful to her for having so assiduously reviewed the document. We also benefited from very helpful comments on the report from Dr. Anrudh Jain, Vice-President, International Programs Division, Population Council, New York.

We also thank Ms. Rolla Khadduri for her useful suggestions on the report. We are most grateful to Pamela Ledbetter for her meticulous review of the report and her editorial inputs. We appreciate Ali Ammad for his hard work on the layout and design of the report. We are thankful to Dr. Najma Lalji from the LSHTM for facilitating coordination between the school and the Population Council. 
Finally, we want to thank the field team leader/medical doctors, social interviewers, laboratory staff, and supervisors who undertook a very challenging assignment, often in very difficult circumstances. We also owe a debt of gratitude to all of the study respondents who willingly gave their time to participate in the study. 



\section{ABBREVIATIONS/ACRONYMS}

\begin{tabular}{|c|c|}
\hline AIDS & Acquired Immune Deficiency Syndrome \\
\hline AKU & Agha Khan University \\
\hline $\mathrm{CBO}$ & Community Based Organization \\
\hline CI & Confidence Interval \\
\hline DFID & Department for International Development [United Kingdom] \\
\hline DBS & Dried Blood Spot \\
\hline ELISA & Enzyme-Linked Immunosorbent Assay \\
\hline FOSW & Female Other Than Sex Worker \\
\hline FSW & Female Sex Worker \\
\hline FBS & Federal Bureau of Statistics \\
\hline HIV & Human Immunodeficiency Virus \\
\hline HSV-2 & Herpes Simplex Virus-2 \\
\hline IEC & Information, Education, and Communication \\
\hline IDI & In-depth Interview \\
\hline IDU & Injecting Drug User \\
\hline IRB & Institutional Review Board \\
\hline LSHTM & London School of Hygiene and Tropical Medicine \\
\hline $\mathrm{MOH}$ & Ministry of Health \\
\hline MSM & Men who have Sex with Men \\
\hline MSW & Male Sex Worker \\
\hline MOSW & Male Other Than Sex Worker \\
\hline $\mathrm{N}$ & Number \\
\hline NACP & National AIDS Control Program \\
\hline NGO & Non Governmental Organization \\
\hline NWFP & North West Frontier Province \\
\hline PCO & Pakistan Census Organization \\
\hline PCR & Polymerase Chain Reaction \\
\hline PM & Partner Management \\
\hline PIN & Personal Identification Number \\
\hline PI & Principal Investigator \\
\hline
\end{tabular}




$\begin{array}{ll}\text { PMRC } & \text { Pakistan Medical Research Council } \\ \text { PSU } & \text { Primary Sampling Unit } \\ \text { RPR } & \text { Rapid Plasma Reagent } \\ \text { RTI } & \text { Reproductive Tract Infections } \\ \text { SIUT } & \text { Sindh Institute of Urology and Transplantation } \\ \text { SOP } & \text { Standard Operating Procedure } \\ \text { STI } & \text { Sexually Transmitted Infection } \\ \text { TPHA } & \text { Treponema Pallidum Hemagglutination Assay } \\ \text { VCT } & \text { Voluntary Counseling and Testing } \\ \text { WHO } & \text { World Health Organization }\end{array}$




\section{EXECUTIVE SUMMARY}

Pakistan currently has very low levels of human immunodeficiency virus (HIV) infection; the overall HIV prevalence rate is less than 0.1 percent in the general population. However, the country is considered to be at high potential risk for an HIV epidemic for a number of reasons. In particular, in the major cities there are large concentrations of individuals engaging in behaviors that make them vulnerable to the rapid spread of HIV and of classical sexually transmitted infections (STIs). The infection can spread to the general population through the bridging population, who are individuals who have contact both with high-risk groups and the general population. The bridging population can be defined by prevalence data of STIs, including HIV, if known, or by the identification of risk behaviors. This study of urban men commissioned by the National AIDS Control Program, with funding from Department for International Development (DFID) (United Kingdom), is one of the three studies originally designed to measure reproductive tract infection (RTI) and STI prevalence and sexual behaviors in the high-risk groups, in men comprising the intermediate-risk group, and in women representing the low-risk groups.

The following were the objectives identified for the study of urban men:

- Measure the prevalence of selected STIs (gonorrhea, chlamydia, syphilis, genital herpes [HSV-2], HIV);

- Measure the behaviors that modify the risk of STI, with an emphasis on sexual behavior and condom use;

- Assess the self-reported STI symptoms and associated treatment-seeking behavior;

- Measure knowledge of STIs, including HIV, and means of avoiding infection;

- Measure perceived risk of STI/HIV infection; and

- Develop a detailed profile and in-depth characteristics and behavioral patterns of the bridging population. 


\section{Methodology}

Six "major urban" cities were purposively selected to include the four provincial capitals -- Karachi, Lahore, Quetta, and Peshawar -- and two of the largest of the remaining cities -- Rawalpindi and Faisalabad. The research involved three distinct research approaches: qualitative, quantitative and biological.

Sixty-four qualitative focus group discussions (FGDs) were held first in the four provinces in the cities of Faisalabad, Karachi, Quetta, and Peshawar during April and May 2007. The FGDs were conducted to establish appropriate terminology for the questionnaire, and to determine the most sensitive approach to the study given the delicate nature of the subject matter of the survey. The second phase of qualitative research consisted of in-depth interviews (IDIs) to obtain detailed information about the sexual attitudes and behaviors of those respondents reporting non-marital sex within the last three months. Researchers conducted a total of 242 IDIs across the six cities, all in the local language.

In the quantitative part of the study, which followed the formative research and was carried out between 1 July and 10 August 2007, 2,400 men were interviewed using a structured questionnaire. Men were the focus of this study as they are thought to be more likely to constitute the bridging population in Pakistan. Ten primary sampling units (PSUs) were chosen in each of the six cities as proportionate to socioeconomic status and high sex ratio. From within each PSU, 40 eligible men were randomly selected from blocks. A total of 400 men were selected through systematic sampling with a random start in each of the six cities. Concurrently, researchers carried out quantitative interviews and the biological component of the study, which tested for the prevalence of five common STIs (namely, syphilis, gonohorrea, chlamydia, HSV-2 and HIV).

A number of measures were instituted to ensure the maintenance of the highest quality standards in both data collection and data analysis. These included: development of standard operating procedures; clear identification of roles and responsibilities for the study team; extensive trainings; careful monitoring, faultfinding and institution of immediate remedial measures; and adherence to ethical principles. 


\section{Results of the study}

\section{Sociodemographics}

The mean age of the respondents in all six cities was 29.1 years. Overall, 15 percent of the respondents had not received any education, and 48 percent were unmarried. The most common language was Punjabi (44 percent), followed by Pashto ( 24 percent) and Urdu (14 percent). Thirty-nine percent of all respondents were within the lowest monthly earning income group of Rs. 1-5,000, while 50 percent reported earning Rs. 515,000 per month. Eleven percent of respondents reported monthly earnings of Rs. 15,000 and above. Within the total sample, 43 percent of the respondents were residing with their parents; the overall population of migrant men (defined as living away from marital or natal home for greater than four months) in the six cities was 9 percent.

\section{Knowledge of STIs/Perception of risk}

Out of the total number of respondents in all six cities $(n=2,400)$, almost all (90 percent) had heard of the term 'HIV/AIDS.' Examining the level of HIV knowledge by educational status suggests a statistically significant difference. Of the respondents who had not heard of the word HIV/AIDS ( $n=240,10$ percent), almost two-thirds (61 percent) had not received any education. Misconceptions about how HIV is spread exist; nearly one-quarter of the respondents mentioned that HIV infection could be spread through the sharing of food (25 percent), clothing (24 percent), bedding (25 percent), and toilets (24 percent) with an infected person. 16 percent mentioned that shaking hands with an infected person could lead to transmission. Overall, only 9 percent of the respondents had been tested for HIV $(n=199)$, and 23 percent considered themselves to be at risk of acquiring a sexually transmitted infection.

\section{Prevalence of STIs}

Out of the total sample of 2,400 respondents, 2,396 agreed to provide blood samples. Out of these 106 (4.4 percent) tested positive for at least one of the five STIs. The prevalence of the individual organisms was as follows: syphilis - 1.3 percent ( 30 cases); HIV - 0.1 percent (3 cases); HSV-2 - 3.4 percent ( 83 cases); gonorrhea - 0.8 percent (2 cases); and chlamydia - (0 cases). The individual collective prevalence of all infections by all five organisms was 4.4 percent, with the highest prevalence in Karachi (8.5 percent) followed by Lahore (5.3 percent), Faisalabad (4.0 percent) Quetta (4.3 percent), Rawalpindi (2.5 percent), and Peshawar (2 percent). 
The total number of STI diganosed was 118 in 106 individuals implying that 12 individuals in the study were suffering from more than one STI. (The collective prevalence of all microoganisms was 5 percent)

\section{Self-reported STIs}

Of the 163 men reporting STI symptoms in the last six months, 91 (56 percent) reported pain during urination. Forty-eight respondents (30 percent) said they experienced ulcer/sores in the genital area and purulent discharge, and 15 percent reported experiencing more than one symptom at the same time. The majority of the respondents sought treatment from medical doctors followed by traditional practitioners.

\section{Non-sexual practices and behaviors}

Overall, about one-third of respondents (31 percent, $n=736$ ) reported ever using any intoxicant. Sixty percent of the total respondents admitted ever having seen pornographic material in their lifetime. Of these, 67 percent mentioned having seen pornography on videos or CDs; among those who reported non-marital sex in the last 12 months, 81 percent had seen pornographic videos or CDs.

\section{Sexual behaviors}

Overall, a little less than half of the men (44 percent) had their first intercourse between the ages of 16 and 20 years. Mean and median age at first intercourse was 22 years. The mean and median age at sexual debut for unmarried men was 18.6 and 18 years respectively. Nearly 29 percent (about one out of every four men) reported having had non-marital sex in their lifetimes. Of these men, 16 percent had premarital sex, while 11 percent reported engaging in both pre- and extramarital sex. Only 2 percent reported exclusive extramarital sex. Regardless of marital status, all respondents who reported ever having sex were asked about condom use during their lifetime. Sixty-eight percent of all respondents $(n=1,643)$ had ever had sexual intercourse. Out of those who had had sexual intercourse, 37 percent $(n=604)$ reported having used a condom at least once in their lifetime.

Fifteen percent of the respondents reported having had non-marital sex in the last 12 months, while nearly one out of every eight respondents reported non-marital sexual activity in the last three months. Non-marital sex was most commonly reported with 
females who were not sex workers ('female friends'); in all six cities the highest proportion of respondents reported having had sex with a female friend. The next most frequently reported category of non-marital partner was female sex workers. Sex with eunuch sex workers (hijra) was less than 5 percent in all six cities.

The analysis across cities shows that 48 percent of the respondents had only one nonmarital sexual partner, 20 percent had two; and 32 percent reported more than three partners in the last 12 months. Out of the total men who reported non-marital sexual intercourse during the last 12 months $(n=370)$, more than two-thirds (68 percent) had 'never' used a condom irrespective of their sexual partner.

\section{Identifying the bridging population}

We applied the common definition of the bridging population as being those individuals who have sexual contact with both high-risk groups and the general population (Aral and Van Dam 1999). In Pakistan, this included married men who reported extramarital sex with a partner from a high-risk group (i.e., male or female sex worker, male other than sex worker, and/or hijra) in the last 12 months and unmarried men who reported sex with two or more partners in the last 12 months with one partner being from a high-risk group. The total bridging population in our sample is 206 .

Across all cities, 141 men in the bridging population reported sexual intercourse with a female sex worker during the last 12 months. The mean age of the bridging population was 25 years and the median age was 23 years; 50 percent had a middle-school education. Fewer married men fell into the category of the bridging population -- 74 percent were unmarried. The results of the multivariate model, which controlled for possible type of partner and marital status, suggest that the bridging population was more likely to include men below the age of 27 years, with less education (having up to ten years of schooling), and who lived with extended family as opposed to joint or nuclear family situations.

Analysis of the entire study population showed that migrant men (those living away from marital or natal home for more than four months) were engaging in non-marital sex at higher rates than non-migrants (37 percent versus 28 percent). However, migrant status was not a significant factor associated with being included in the bridging population, which suggests that migrant men are having non-marital sex with partners other than sex workers. 


\section{Discussion}

This study is unique in terms of its subject matter (sexual behaviors and attitudes); its study population (urban men from the general population); and also its methodology (biological testing for STIs in conjunction with a detailed behavioral questionnaire and in-depth qualitative research). It identifies the potential bridging population within the existing population of urban men. Furthermore, it helps to assess men's perceptions, knowledge, and sexual behaviors as they relate to men's risk for STI transmission.

Importantly, the behavioral and qualitative components of the study identify risky sexual behaviors that may have important implications for the future trajectory of the epidemic. The first has to do with rates of premarital and extramarital sex reported in the study. Twenty-nine percent of men reported that they had engaged in either premarital or extramarital sex in their lifetime. This contradicts the widely held notion that religious and sociocultural norms are sufficient for containing the HIV epidemic in Pakistan. This study shows that non-marital sexual activity is taking place and that a smaller proportion of men engage only in extramarital sex. While premarital sex is not necessarily risky behavior, the fact that most men who engage in extramarital sex also reported premarital sexual behavior suggests the potential for men to begin risky concurrent sexual relationships prior to marriage. The reasons for this remain unclear, but it does suggest that further research should be conducted to investigate this difference, and that interventions should target unmarried men as their sexual risk behaviors begin before they are married.

Furthermore, this study shows that premarital sexual activity among urban men in Pakistan begins at young ages. With the increasing age of marriage, the period of sexual opportunity is widening, allowing more opportunities for risky sexual behavior. The study results suggest that as men grow older and have greater financial resources at their disposal they are more likely to access sex workers. The qualitative data suggest that within the younger age groups, peer pressure and exposure to the media greatly influence sexual behaviors. Some of the risky sexual behaviors that youth currently engage in are reported in the qualitative findings; these are learned through exposure to pornography in videos/CDs and/or the Internet.

Another phenomenon identified by the qualitative study component, and which needs more elaboration, is related to having sexual liaisons with so-called 'friends' who are identified through mobile telephones. Such relationships are often described as 
monogamous; however, it remains ambiguous if these 'friends' have other partners as well.

The study also points out the variation in STI prevalence across cities that is linked to the demographic characteristics obtaining in each city. Karachi had a high STI prevalence of 8.5 percent. The prevalence of HSV2 infection of 6 percent was also the highest in Karachi.

The study shows that while most men have heard of HIV/AIDS and some of the modes of its transmission, this knowledge has not been internalized sufficiently, as men still do not consider themselves to be at risk even while engaging in risky behavior. Misconceptions regarding HIV transmission also exist. Condom use was reported to be generally low in both marital and non-marital unions, stressing the need for further analysis of the reasons for this, especially factors related to condom availability and quality.

The qualitative research findings show that many STI patients still feel more comfortable visiting traditional practitioners for their care, owing to issues related to privacy and confidentiality. The quantitative data also show that in cities, such as Lahore and Karachi, traditional practitioners were favored more when respondents were seeking care for conditions such as purulent discharge and pain during urination. Access to services within the public sector can be improved through the provision of high quality STI care throughout all levels of the health care system, including basic health units and rural health centers. A positive finding of the study has been the almost universal awareness regarding the use of safe blood and the need for prior testing of blood before it is transfused. Our study also found very low drug use among the study population. 


\section{Recommendations}

The recommendations that emerge from this study suggest a three-pronged holistic approach that addresses behavior change through information-educationcommunication (IEC) strategies; provision of health services (health care delivery); and monitoring of progress (monitoring and evaluation).

\section{Behavior change communication program}

An awareness raising and sensitization strategy on protection against STIs that adopts a targeted approach that reaches different segments of society, especially the youth, needs to be introduced. Precise information of all factors that determine the risk of STI/HIV transmission needs to be elaborated, while also mitigating the stigma attached to it. This can be done by utilizing several approaches, such as introducing life skills education programs in schools, health-education campaigns in colleges, universities, and in the workplace. Behavior change can only take place if credible information is made available to the people and is periodically reinforced. The focus of all IEC campaigns needs to be directed at risky behaviors, such as reducing the number of sexual partners, emphasizing the importance of monogamous relationships, increasing condom use and delaying sexual debut.

\section{Health care delivery}

Access to integrated reproductive health services needs to be enhanced by ensuring the availability of comprehensive and integrated reproductive health services within all tiers of the health system. It requires a number of initiatives, that begin with change in the medical curriculum that emphasizes a holistic and integrated approach in identifying and meeting the reproductive health needs of all age groups and in improving the quality of existing STI care being offered within both public and private-sector facilities, through in-service refresher trainings that focus on counseling, diagnosis and management of STIs, as well as the importance of maintaining privacy and confidentiality. With additional trainings, most of the existing health facilities can manage STI patients, and also provide youth-friendly services. In addition, there is also a need for increasing the number of voluntary counseling and testing services within large urban communities and informing the public about their location and the services that are offered. 


\section{Monitoring and evaluation}

Ensuring that reliable information related to STIs is provided in a timely manner is an important step in reducing the likelihood that an STI epidemic reaches the general population. It is necessary to put in place mechanisms that can provide reliable and up-to-date information on sexual behaviors and STI prevalence, such as institutionalizing the second-generation surveillance system and expanding it to reach a wider number of cities of the country. Establishing sentinel surveillance mechanisms within the major urban canters can also help to inform the public health system of the incidence of STIs in a timely manner, enabling a prompt response. In addition, surveys on the bridging population need to be replicated periodically to gauge trends over time. Using selected indicators, such as the use of condoms and the extent of concurrent partnerships, these surveys can measure the impact of specific programmatic interventions. 



\section{Background}

Pakistan currently has very low levels of human immunodeficiency virus (HIV) infection; the overall HIV prevalence rate is less than 0.1 percent in the general population (World Bank 2006). However, the country is considered to be at high potential risk for an HIV epidemic for a number of reasons. In particular, in the major cities there are large concentrations of individuals with behaviors that make them extremely vulnerable to the rapid spread of HIV and of classical sexually transmitted infections (STIs), such as chlamydia, syphilis and gonorrhea (Akhtar et al. 2000; Ahmed et al. 2003; Baqi et al. 1999). These individuals include male and female sex workers (MSWs and FSWs); men who have (unprotected) sex with men, transsexuals (hijras) or eunuchs; injecting drug users (IDUs); and highly mobile occupational groups such as truckers (Agha et al. 2003; Agha 2002; Ahmed et al. 2003; Baqi et al. 1999).

Recent evidence suggests that Pakistan is facing a concentrated epidemic in these highrisk groups with HIV prevalence among some groups already surpassing five percent (World Bank 2006). The infection can spread to the general population through individuals who have contact with both the high-risk groups and the general population. In epidemiological parlance, individuals who spread the infection from concentrated high-risk groups to the general heterosexual population are termed the bridging group (or bridging population) (Morris et al. 1996; Gorbach et al. 2000; Lowndes et al. 2002). Typically, these are married or unmarried men who are clients of sex workers, or who are bisexuals, or who share needles with injecting drug users. Little is known about the sexual conduct of men and women in Pakistan, but the available evidence suggests that both premarital and extramarital sexual contacts either homosexual or heterosexual - may be sufficiently common to create and sustain a generalized HIV epidemic (Faisel and Cleland 2006; Agha 2002; Khan and Hyder 1998). There is less understanding about female sexual behaviors in Pakistan, which limits the ability to fully understand the potential for a generalized HIV epidemic in the country. 
The potential for bridging populations to transmit HIV and other STIs through their sexual behavior is thus a significant concern for the Ministry of Health and the National AIDS Control Program (NACP). Identifying the bridging population and bridging behaviors among urban men and understanding their levels of knowledge and behaviors related to STIs and HIV is critical for slowing the potentially rapid spread of HIV in Pakistan.

A consortium of organizations including the Ministry of Health, the DirectorateGeneral Health, NACP, the London School of Hygiene and Tropical Medicine (LSHTM), the Population Council, and the Aga Khan University (AKU) designed the National Study of Reproductive Tract Infections and Sexually Transmitted Infections in Pakistan (LSHTM, Population Council, and AKU 2002). The work was done with the financial support of the Department for International Development (United Kingdom) (DFID). The proposed national study included three separate studies for measuring prevalence of selected STIs and assessing sexual behaviors within the highrisk groups, the bridging population, and the general population. This report describes the findings from the survey of the bridging population, which was conducted by the Population Council in six cities of Pakistan.

The bridging population can be defined by prevalence data of sexually transmitted infections, including HIV, if known, or by the identification of risk behaviors. There has been limited research on the bridging population in Pakistan and there is no definitive evidence (either prevalence data or behavioral risk factors) to define the bridging population as a single category or a sub-population (e.g., men who buy sex or truck drivers). Most of the HIV and STI prevalence data in Pakistan comes from studies in high-risk groups or in single cities, which are not useful for generalizations. For example, studies in Pakistan have been carried out among migrant men (Faisel and Cleland 2006), blood donors, injecting drug users (Ahmed et al. 2003; Emmanuel et al. 2004), long distance truck drivers (Agha 2000), prison inmates (Akhtar et al. 2000), university students, hijras or eunuchs (Baqi et al. 1999), and female sex workers (Nanan et al. 2000).

A study identifying the characteristics of the bridging populations in major urban centers of the country and investigating their bridging behaviors was considered to be an important contribution to the design of appropriate STI and HIV interventions in Pakistan. The results from this study address the existing gaps in knowledge and 
understanding related to STIs and HIV among the bridging population of urban men in Pakistan.

\section{Context}

Before commencing the study, an extensive review of international and national literature was carried out to inform and further refine the study design. This study was designed to allow for a broader definition of the bridging population that captured men already thought to be the bridging population (e.g., migrant men and 'men on the move'), but also allowed for better understanding of the potential bridging population among urban men. Some of the main findings of the literature review relevant to the study of the bridging population are summarized below.

\section{Homogeneity}

The literature related to the bridging population is suggestive of the wide range of risk behaviors and the important role of contextual factors in determining how the bridging population is defined. Current understanding of risk factors in Pakistan does not definitively point to a specific population as being the bridging population. Several studies from Pakistan suggest a crossover of risky behaviors that may blur the definition of the bridging population. One such example is men who have sex with men (MSM) but who are also married or engage in sexual activity with a female other than a sex worker (Khan and Khan 2006; Khan and Hyder 1998). A study that explored the sociocultural dimensions surrounding sex between men of South Asian descent in the United Kingdom and the United States reported a high number of male sexual partners among male respondents of all ages. The majority of these male respondents were married. The lack of accessibility to women and strong cultural taboos against extramarital heterosexual activity were identified as cultural factors contributing to men who have sex with both men and women (Khan and Hyder 1998). A study conducted in India (Dandona et al. 2005), found that 42 percent of MSM sampled from highly visible locations of MSM activity in Andhra Pradesh were currently married. These MSM could therefore be considered to be a part of the bridging population. 


\section{Migration and mobility}

The migrant population is defined as those men or women who spend significant periods of time away from their marital or natal families. Typically, these migrants are moving from rural to urban areas, most often for employment opportunities (Choi et al. 2004; Coast 2006). Migration and social movement and their impact on the spread of HIV have long been acknowledged. The migrant population is seen as a group that may engage in risky behaviors, such as drug use or sexual activity, in response to the loneliness and stress of being in a new environment where they may be unsupervised and unsupported (Mann and Tarantola 1996; Parker et al. 2000).

Several studies of migrant men suggest there is the potential for a group or social norm effect that may push migrant men to partake in risky behavior that they might not otherwise choose. For example, Hawkes and Santhya (2001) found that men often go to sex workers in groups; a study in Cambodia (Sopheab et al. 2006) found that military men were more likely to go in groups to purchase sex from sex workers than they were to go individually.

Faisel and Cleland's (2006) behavioral and biological study assessed sexual risk behavior and the prevalence of treatable STIs in migrant men in Lahore. (They defined migrants as men living 50 percent of the past six months away from their natal or marital home.) They carried out behavioral interviews with 590 migrant men aged 2049 years and collected biological samples of a sub-sample of 190 men to test for chlamydia, gonorrhea, and syphilis. Their behavioral study findings suggest high rates of premarital sex among the migrant men: 53 percent of respondents were single and of these men, 55 percent reported having had sexual experience. Forty-eight percent of the single men reported sexual activity with a female friend/relative and 22 percent reported sex with a female CSW. Only 10 percent of migrant men in the study used condoms with sex workers; with male partners condom use was rare. The biological study finding was a 3.2 percent prevalence of STI infection (i.e., chlamydia, gonorrhea, and syphilis) among the 190 migrant men sampled.

The literature also suggests it is not just the migrant population but also mobile men who are a potential bridging population (Hawkes and Hart 1993). It is thought that those who travel may be more prone to engage in risky behaviors while traveling and that mobility increases the potential for casual contact with multiple sexual partners or interactions with CSWs. Sopheab et al. (2006) found that mobility (defined as being away from home for more than one month in the past year) significantly increased the 
likelihood (to about two times) of casual sex among men. Furthermore, men who are mobile may serve as a bridging population for HIV and STI transmission not only between two genders but also between two or more geographic areas.

\section{Occupational status}

Occupational status is an important factor to consider when assessing risk behaviors and the bridging population. Both men who are mobile and migrants are typically identified by occupational characteristics, such as those working in the transport industry (i.e., taxi drivers in Philippines [Morisky et al. 2005] and long distance truck drivers [Chen et al. 2006]), or middle-class and wealthy businessmen in China (Lau and Thomas 2001), or construction or factory workers (Puri and Cleland 2006).

A well-documented example of occupational status as a risk factor for the transmission of HIV is long-distance truck drivers (Agha 2002; Chandrasekkaran et al. 2006). Several of these studies suggest truckers are more likely to be clients of female sex workers than the general population; 24-34 percent of truck drivers in India reported sex with a CSW in the previous year (Chandrasekkaran et al. 2006). Studies have also found unpaid sexual activity between truck drivers and their male helpers (Family Health International 2001; Chandrasekkaran et al. 2006). Sohail Agha's study (2002) in Pakistan examined self-reported sexual behavior and condom use among truck drivers and their helpers at truck stands in Lahore. His study found that it was common for long-distance truck drivers to engage in high-risk sexual practices: 60 percent of truckers had ever paid for sex with a man or a woman. This study also found that while 11 percent of men reported having had sex with a male sex worker (MSW), 49 percent of men reported having had sex with a man who was not a sex worker.

Other results from the literature suggest variations in risk behavior according to occupation. In the Hawke et al. (1999) study in Matlab, Bangladesh, women married to casual workers had an increased risk of STI acquisition. Faisel and Cleland's (2006) study of migrant men in Lahore found variation in reporting of non-marital sex by occupation: skilled workers, house servants/drivers, business or salesman were more likely to report non-marital sex.

\section{Extramarital and premarital sex}

As noted by Schensul (2006b), "men's extramarital sexual behavior is the most important factor for introducing HIV/STI into marital life" (page 615). Studies in the 
South Asian context have found high rates of both extramarital and premarital sexual activity. Collumbien's (2000) study of urban and rural men in India found that 25 percent had sex before marriage and with a variety of premarital sex partners (e.g., sex workers, friends, relatives, and future spouses). Hawkes and Santhya (2000) review of the literature on HIV in India reports that studies in the general population found premarital sexual activity among men to be between 7 and 48 percent. As mentioned before, 55 percent of the unmarried men in the study of migrant men in Lahore reported sexual experience (Faisel and Cleland 2006).

It is important to note that not all extramarital sex is with sex workers. Examples from the literature include the 48 percent of men in the Faisel and Cleland (2006) study who reported sex with a female friend or relative; the married truckers who have sex with men but do not pay for it in Sohail Agha's study (2002); and the increased importance of unpaid sexual activity in HIV transmission in Cambodia (Gorbach et al. 2000).

\section{Variability of STI and HIV transmission in different cultural contexts}

There is discussion in the literature as to whether certain countries will face similar HIV/AIDS epidemics as countries in Sub-Saharan Africa have (Caldwell 2006; Gray 2004; Godwin et al. 2006). While there is no definitive evidence, some have argued that culturally proscribed behaviors (i.e., male circumcision, prohibition against homosexuality and extramarital sex, prohibition of alcohol consumption, and proscription of female sexual behaviors) may provide protection against HIV transmission in some countries (Gray 2004; Caldwell 2006). There is increasingly strong evidence that male circumcision is protective against the transmission of HIV (Auvert et al. 2001a and 2001b; Auvert et al. 2005). John Caldwell (2006) suggests that the lack of male circumcision in the majority of the population of India explains its higher HIV levels as compared to Pakistan and Bangladesh. Peter Gray's (2004) study of a sample of Sub-Saharan African countries found that a higher percentage of Muslims in a country was a negative predictor of HIV prevalence. Peter Godwin et al. (2006) states that Asia will not experience generalized HIV epidemics because of the tight social and cultural controls on female's sexual behavior in many Asian countries. However, the literature cautions that other risk behaviors may offset the protective effects of male circumcision (Gray 2004). In-depth studies of the risk factors and behaviors related to HIV transmission in countries where circumcision is commonly practiced is needed. 


\section{DESCRIPTION OF STUDY}

\section{Study objectives}

The following is a list of the objectives identified for the study.

- Objective 1. Measure the prevalence of selected STIs (gonorrhea, chlamydia, syphilis, HSV-2, HIV).

- Objective 2. Measure the behaviors that modify the risk of STI with an emphasis on sexual behavior and condom use.

- Objective 3. Assess the self-reported STI symptoms and associated treatmentseeking behavior.

- Objective 4. Measure knowledge of STIs, including HIV, and means of avoiding infection.

- Objective 5. Measure perceived risk of STI/HIV infection.

- Objective 6. Examine variation in the above across major strata of this population.

- Objective 7. Develop a detailed profile and in-depth characteristics and behavioral patterns of the bridging population.

\section{Study population}

As described earlier the study was commissioned to look at STI prevalence and behaviors in urban men who could act as a bridge for spreading STIs from the high to the low-risk groups. The study population was urban men aged 16-45 years residing in the study area who had been resident in the selected household on the night prior to enumeration. The age band took into account the near-universal finding that men's risk behavior declines from about age 30 and reaches very low levels by age 50 (Carael et al. 1995). The study population included both married and unmarried men from different occupations (including those working in the informal sector) and those who 
were students or unemployed. It represented most linguistic groups since migration to cities is likely to originate from almost all parts of the country.

Six major urban cities ${ }^{1}$ were purposively selected to include the four provincial capitals (Karachi, Lahore, Quetta and Peshawar) and the other largest cities of the country (Rawalpindi and Faisalabad). These study sites provide information about men from all across Pakistan. For example in Karachi, men were found who belonged originally to North West Frontier Province (NWFP), Azad Jammu Kashmir (AJK), Balochistan, Punjab, as well as interior Sindh. Altogether, the population of these six cities represents 85 percent of the population of the ten largest cities in Pakistan (Pakistan Census Organization 1998).

\section{Study design}

The research involved three distinct research approaches: qualitative; quantitative; and biological. Each approach addressed the same topic from a different perspective and study methodology. Triangulation of the results during final analysis allowed for a more comprehensive understanding of the subject matter. The sequencing and timing of the three research approaches is depicted in Table 2.1.

Table 2.1: Sequencing of research for survey of the bridging population

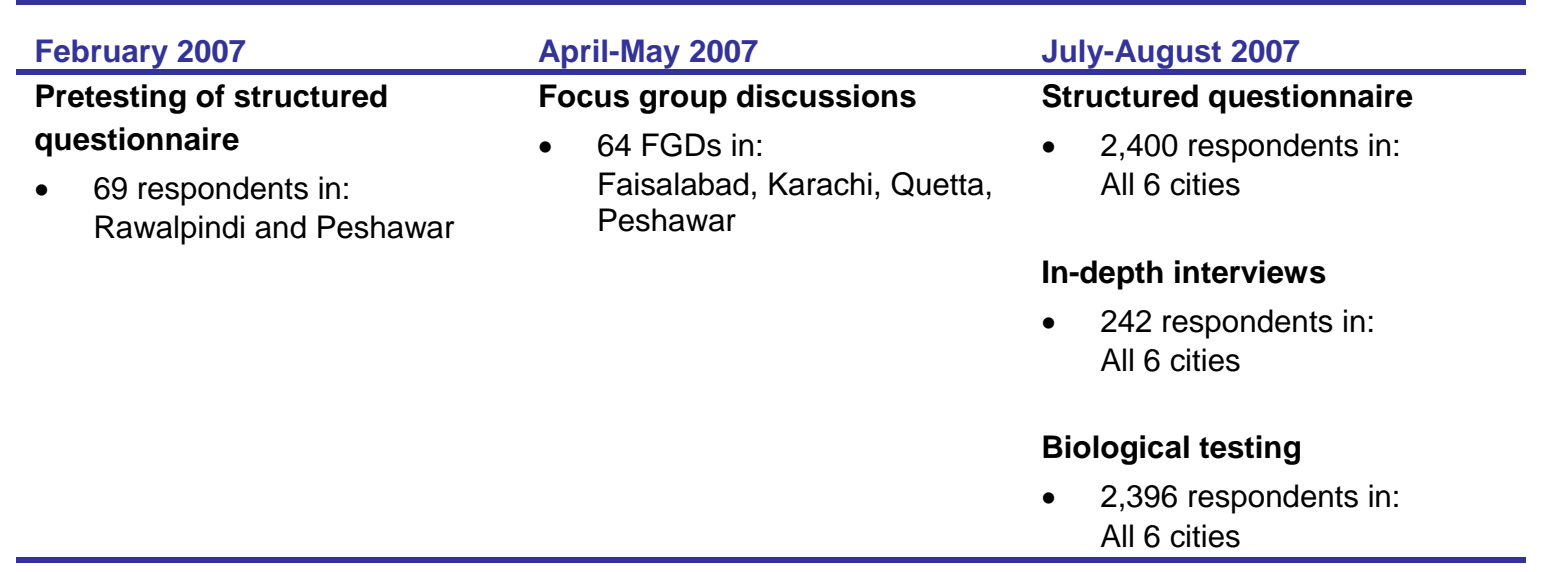

\footnotetext{
${ }^{1}$ Following the definition used by the Federal Bureau of Statistics.
} 


\section{Qualitative research}

Focus group discussions. Qualitative focus group discussions (FGDs) were held first in the four provinces of Faisalabad, Karachi, Quetta, and Peshawar. The Population Council contacted key informants (such as influential community leaders identified by the medical colleges in each of the four cities), who introduced the researchers to the communities and helped with the selection of FGD participants. Each FGD was conducted by two trained social scientists (a moderator and a note-taker) and included six to eight participants. Informed consent was obtained from all participants. The social scientists used a topic guide for the FGDs to ensure they covered all of the relevant topics. FGDs took an average of one-and-a-half hours; each was conducted in the local language and tape-recorded. Researchers conducted a total of 64 FGDs during the months of April and May 2007. The purpose of the FGDs was to establish the most appropriate terminology for the behavioral questionnaire, determining the most sensitive approach to the study, given the delicate nature of the subject matter. (See appendix 7 for further details on the methodology of the FGDs and the focus group discussion guide.)

In-depth interviews. The second phase of qualitative research consisted of in-depth interviews (IDIs) to obtain detailed information about the sexual attitudes and behaviors of respondents. These IDIs were not part of the formative research but were carried out with those respondents who, on the behavioral questionnaire (quantitative component of the study), reported having non-marital sex in the last three months. A total of 242 IDIs were conducted across the six cities, all in the local language. Separate informed consent was obtained from the respondents. Each IDI took approximately 40 minutes and followed a basic set of topics, with probes when appropriate. (See appendix 6 for the IDI guide.) The IDIs were carried out either immediately following the behavioral questionnaire or at a mutually convenient time scheduled a day or so after the behavioral questionnaire. The focus group guidelines and in-depth interview checklist were finalized after a rigorous consultative process.

\section{Quantitative research}

Behavioral questionnaire. The quantitative portion of the study, which followed the formative research (carried out between 1 July and 10 August 2007), reached 2,400 men who were interviewed using a structured questionnaire (see appendix 10 for the 
questionnaire). Ten primary sampling units ${ }^{2}$ (PSUs) were chosen in each of the six cities as proportionate to socioeconomic status and high sex ratio of more than 120 . The proxy indicator for socioeconomic status was female literacy, which has been used in other studies and is considered a good indicator of socioeconomic status (Filmer and Pritchett 1998; Rutstein and Johnson 2004). From within each PSU, 40 men were selected systematically with a random start. A total of 400 men were randomly selected in each of the six cities, resulting in a total sample size of 2,400 men.

Block demarcation was based on geographical mapping provided by the Pakistan Census Organization (PCO). A team of enumerators visited each of the selected blocks a few days prior to data collection. According to the sampling frame provided to them, they marked each selected structure (house, mosque, shop, school, hostel, or any other place where a person might live) in the selected PSU and completed a form. From each structure, information about the number of persons living in the structure (both male and female members) and men living away from their marital and natal home was collected. Structures were marked/numbered so that none of the structures were missed or duplicated and so that selected structures could be easily identified during the data collection phase. Information about locked structures was obtained from neighbors. In the case of hostels and hotels, each room was treated as a structure and persons living there were listed per room. During this listing process, sites for establishing a temporary clinic and laboratory were also identified. For this purpose local area leaders (nazims and councilors) were contracted, and their help proved highly useful.

Male interviewers specifically trained in social sciences gathered social, demographic, and behavioral information during structured interviews with eligible men using the structured questionnaire. The questionnaire was developed based on comparable international survey tools (Family Health International 2000). Comments on the questionnaire were also solicited from both national and international experts having extensive experience in the field. The questionnaire was subsequently translated into Urdu and retranslated into English to ensure consistency. The final version was in both English and Urdu. The questionnaire was pilot tested and consequently adapted on several occasions. Researchers pretested the structured questionnaire on 69 respondents - 40 in Rawalpindi and 29 in Peshawar.

\footnotetext{
${ }^{2}$ The PSUs were obtained from the Population Census Organization.
} 
Researchers conducted a total of 2,400 structured questionnaires. Each questionnaire took, on average, 40 to 45 minutes to conduct. Each interviewer collected information from three to five men in a day. The structured questionnaire was administered between 3 p.m. and 9 p.m.: the hours thought to be the most likely to find eligible men at home.

\section{Biological testing}

Concurrent with the quantitative interviews, the researchers carried out the biological component of the study on the 2,396 men. Four respondents refused providing their blood samples after participating in the behavioral interview. An important aim of the study was to assess the prevalence of STIs among the study population. In order to do this, detection of STIs through blood and urine tests was carried out. Arrangements for privacy and confidentiality were ensured, as detailed in the ethical considerations section of this report. Researchers tested for syphilis, gonorrhea, chlamydia, HIV, and herpes simplex virus two (HSV-2). The collected specimens were marked with a bar code having a personal identification number (PIN). A copy of the PIN was handed to the study subject for retrieval of his test results at a later date.

All participants were informed about the study team doctor and were given the option to consult him for their reproductive health, or even general health, concerns. The doctor provided treatment for STIs based on WHO syndromic management guidelines, including information on partner notification and follow-up. Based on the rapid results for syphilis testing carried out on the spot by the lab technician, the doctor also provided treatment for syphilis, as necessary. In addition, the doctor provided general information on voluntary counseling and testing centers (VCTs) in the area, and the range of services provided by these centers.

Table 2.2: Biological tests undertaken in survey group

\begin{tabular}{lcr}
\hline Microorganisms & Sample required & Tests performed \\
\hline Chlamydia & Urine & PCR (Roche Amplicor CT) \\
Gonorrhea & Urine & PCR (Roche Amplicor NG) \\
Syphilis & Blood & RPR/TPHA \\
HSV-2 & Blood & ELISA \\
HIV & Blood & ELISA \\
\hline
\end{tabular}

The study design incorporated testing for the five STIs listed in table 2.2 for several reasons. Syphilis is one of the most common STIs globally, but estimates of syphilis 
prevalence in the general population in Pakistan are not available. Despite being easy and inexpensive to detect and treat, it remains an important contributor to the overall disease burden in developing countries. Being an ulcerative condition, it also increases the risk of transmission of HIV (Cameron et al. 1989). Syphilis testing through blood was carried out in the field using the Determine rapid test (immunochromatography). It was confirmed in the laboratory through the rapid plasma reagent (RPR) and Treponema pallidum hemagglutination assay (TPHA) confirmatory tests. The TPHA confirmatory test measures both past and active syphilis.

Chlamydia and gonorrhea are considered to be fairly common STIs. Gonorrhea has been reported as the leading cause of consultation in a STI clinic in the NWFP (Khan et al. 1995). However, as the presence of both chlamydia and gonorrhea can be detected in urine only within three months from the time of infection, it was recommended that only those respondents reporting non-marital sex in the last three months be included in the biological testing for chlamydia and gonorrhea. Samples were pooled (ten sera were pooled) and tested for chlamydia and gonorrhea using polymerase chain reaction (PCR) testing (a technique already commonly used in Pakistan). A number of international studies, including a meta-analysis, have indicated that previous genital HSV-2 infection is a risk factor for the spread of HIV (Stamm 1988; Wald 2002). There are few studies from South Asia that determine HSV-2 prevalence, hence its inclusion in this study. HSV-2 was tested from blood samples. The protocol for the biological sampling of urine and blood is found in appendix 8 .

Out of the total 2,396 blood samples send to SIUT for testing results for 2383 samples were received, 274 urine samples were sent and 256 were received

\section{Sampling protocol and sample size}

The sample size calculations were based on a conservative expected 7 percent prevalence of any tested STI (chlamydia, gonorrhea, or syphilis). Expected compliance for the biological data collection was 70 percent, based on the pretest results. The design effect (which takes into account the increase in standard errors due to clustering) was set at 2.0. This value reflected the expected geographic heterogeneity of infection and behavior in urban areas, and was based on experience with standard errors estimated for urban strata in demographic surveys (Verma et al. 1980).

The proposed sample size of 2,400 would have the power to detect a decrease in STI prevalence from 7.0 percent to 4.4 percent if the study was repeated in five years, for 
instance. For an estimate of 3 percent (e.g., for a specific STI), the confidence interval would be 2.1 percent - 3.9 percent. The proposed sample size can also be justified in terms of its power to detect behavioral change with statistical confidence.

Sample surveys of men inevitably record lower participation than surveys of women, because men are harder to trace at home and more likely to refuse to participate in the study (Mishra et al. 2006). For example, the 1991 Pakistan Demographic and Health Survey had a 77 percent response rate for males. As the STI study expected limited participation from men, interviewers were instructed to work in the evening and to make at least three return visits to each dwelling.

The sampling protocol was a stratified, multistage systematic sample of households. Keeping in view the heterogeneity of these cities, the sampling protocol aimed to capture the full range of the socioeconomic conditions of the city population. For this purpose, the literacy rate and the sex ratio, obtained from the 1998 Population Census, were used as the indicators of the socioeconomic status for each of the census enumeration circles. The "enumeration circle" is the smallest unit available in the 1998 Population District Census as demarcated by the population census organizations for these cities.

In the first stage, ten enumeration circles were selected with probability proportional to size. The maps of these circles were obtained from the Population Census Organization. In these circles, the areas are already divided into blocks of approximately 250-300 households depending on the number of households in each circle.

The second stage was to randomly select one block with probability proportional to size from the list of total blocks from each of the ten circles. The enumeration teams updated the listing of each block before selecting the sample of households from each block. The third stage involved the selection of 40 households from each PSU using the systematic random technique.

At the fourth stage, one eligible respondent (male resident aged 16-45 years; resident in household the previous night) was selected randomly from the household roster and was asked to participate in the study. The selected respondent was given the option of being interviewed at his home or at a preselected private location in the same block. 
In order to measure STI prevalence, a sample size of 2,400 was required and therefore a systematic replacement strategy was developed. Replacement was done systematically at both the household level and the individual level. In the first instance, selected households were replaced with adjacent households if:

- The selected household was locked and it was confirmed that the household member was not available during the time the study team was to be in the area;

- The selected household did not have any eligible male member; or

- The respondent was not available after three visits during the study team's stay.

The selected individual was replaced with another eligible male from the same household if:

- The selected respondent was not available during the study team's stay in the block; or

- The selected respondent was sick or handicapped.

If the selected respondent refused to participate in either the structured questionnaire or the biological component of the study, the entire household was replaced.

\section{Study team}

The Population Council was responsible for study team selection. Sindh Institute of Urology and Transplantation (SIUT) facilitated in the training of the medical doctors, phlebotomists and lab technicians. Each member of the study team had clearly defined roles and responsibilities, as detailed below.

Medical doctor. The study team medical doctor headed the field team and was based at a central location of the PSU, such as the union council office, or a room in the local college. He was responsible for overall supervision, quality assurance (including storage and transport of the biological samples), and all field activities undertaken by the study team. He interpreted the rapid syphilis tests during the biological research component, provided general and syndromic management of presumptive STI cases, provided counseling and advice on partner management and, if needed, referrals to the nearest VCT services. The medical doctors were hired from the premier academic institutions of the country. 
Phlebotomist. The phlebotomist was responsible for the collection of blood and urine samples. He collected the samples according to the protocol developed by SIUT. He handed over the samples to the lab technician. He also affixed the personal identification number (PINs) to the containers and gave a laminated identification card with the PIN number to the respondent, which was later used for the identification of laboratory results. Both the phlebotomist and the lab technician were fully qualified and were selected from the private and public sectors.

Lab technician. The lab technician was responsible for the maintenance of quality standards and adherence to standard operating procedures. He also maintained the $\log$ entries in a special register. He carried out the RPR rapid test for syphilis and prepared the dried blood stain (DBS) cards. He also prepared the specimens for transportation.

Social science interviewer. The social science interviewers conducted the FGDs, IDIs, and structured questionnaires; they also provided information on the nearest VCT centers and general information on HIV/AIDS. Almost all of the social science interviewers had a master's degree in either sociology or population sciences. Four social science interviewers were medical doctors. Almost all social science interviewers were young; none of them were above the upper age limit of the respondent.

\section{Data management/Data analysis}

The team leaders sent the completed quantitative questionnaires to the Population Council office in Islamabad after completing each block. Prior to commencing data entry, the questionnaires were edited. The data were double-entered, and range skip and filter checks were applied to minimize errors and omissions. The data were then coded and entered into the computer using CSPro (version 3.2). SPSS (version 14) was used for quantitative data analysis. All study data and forms were stored in locked cabinets at the Population Council office in Islamabad. Qualitative data were analysed manually. After a thorough content analysis, codes were given and a matrix was developed and themes identified. Data were sorted based on themes and sub-themes and analysed manually. 


\section{Quality assurance}

A number of measures were instituted to ensure the maintenance of the highest quality standards in both data collection and data analysis. These measures are described below.

\section{Standard operating procedures}

The SIUT developed standard operating procedures for carrying out all the laboratory processes. These procedures ensured that specimens were collected under aseptic conditions and with minimal discomfort to the patient, and that proper storage and the cold chain was maintained during transportation.

Determination of roles and responsibilities. Roles and responsibilities of each team member were clearly identified. Each member was provided with a written set of responsibilities that they were required to follow.

Training. The social science interviewers received seven days training conducted by the Population Council. The training included sessions on the research protocol, ethics, obtaining informed consent, maintaining privacy during the interview process, sampling methodology, and interviewing techniques. Numerous role-play sessions were conducted as part of the questionnaire training. The training stressed the importance of respecting respondents and adopting a nonjudgmental attitude throughout the interview. One day of practical training in the field was included and allowed for individual feedback for each interviewer. The trainees were informed about zero tolerance for any moral or ethical dishonesty and the importance of maintaining confidentiality of the information they obtained.

The medical doctors received four days of training, conducted by Dr. Sikandar Sohani, Health Advisor in Aahung and a renowned specialist in STIs. Dr. Sohani provided a refresher course on the syndromic management of STIs, partner management, obtaining a sexual history, and providing client-centered services. Finally, the lab technicians and phlebotomist received two days of training conducted by SIUT. The training focused on how to adhere to the standard operating procedures, as well as familiarizing the participants to the study. 


\section{Monitoring}

During the fieldwork the principal investigator, study coordinator, and deputy study coordinator (all from the Population Council) regularly visited each study site. They randomly selected ten percent of the respondents to ensure that the sampling procedures and all standard operating protocols were being followed. In particular, the monitors checked whether the respondents had received printed and verbal information on HIV/AIDS, their personal identification numbers, and information on VCT centers. During these visits, the monitors also randomly selected completed questionnaires to check for completeness and accuracy. The final component of the field monitoring was to observe laboratory procedures and to provide on-the-spot guidance as needed. The monitors filled in monitoring forms (a sample of which can be seen in appendix 9), and prepared a report of each visit.

Fault finding and remedial measures. Through the process of ten percent validation of all respondents, the monitoring teams identified some instances of sample violations and immediately addressed them. For example, the monitors discovered that in Lahore the social science interviewers were not adhering to random sampling techniques. The entire team of researchers was terminated and the data discarded. New researchers then repeated the fieldwork in the four PSUs under the direct supervision of the monitors.

\section{Ethical considerations}

Ethical approval was obtained from three institutional review boards (IRBs): HOPE, a local organization in Pakistan which has a recognized ethical review board; the Population Council's headquarters in New York; and the London School of Hygiene and Tropical Medicine in London (copies of approval can be seen in appendix 2). The steps taken to ensure that the study met all ethical considerations are described below.

\section{Informed consent}

Informed consent was obtained from all study participants after describing to them in detail the issues related to the study. Informed consent was obtained from parents and guardians of respondents aged 16 and 17 years. Social science interviewers obtained separate informed consent for the qualitative components of the study (the FGDs and the IDIs). For the structured questionnaire, the social science interviewers described the scope and purpose of the questionnaire and its approximate length, and they 
stressed that participation was entirely voluntary. The respondents were further informed that participation in the interview did not necessitate participation in the biological data collection.

Next, the social science interviewers gave a more detailed description of the biomedical component. Social science interviewers described the specimen collection procedures and their purpose as well as the opportunities for treatment by the doctor on-site in case of syphilis detection, and secondary treatment when the laboratory results for other STIs became available. The confidentiality of all medical results was stressed. Participants were given four options to confirm their informed consent: consent by signature; consent by fingerprint; oral consent with a witness; or oral consent recorded by audiotape.

\section{Privacy}

The structured questionnaires were conducted in private and out of the hearing of other people. When these conditions could not be obtained, social science interviewers offered participants an alternative venue or time to complete the questionnaire. At the field centers in each PSU, adequate auditory and visual privacy was ensured, particularly for syndromic management and counseling.

\section{Confidentiality}

All data collected in each phase of the study was kept confidential and anonymous. The structured questionnaires and in-depth interviews were identified by personal identification numbers rather than participant names. If the respondent was also participating in the biological study, the same PIN was attached to the urine sample, the blood sample, and the dried blood stain (DBS) card. Finally, the same PIN was attached to a plastic card that was given to the respondent to be used for identification purposes when picking up his results. The medical doctor also assured all participants of his adherence to the medical code of patient confidentiality when discussing the results of the rapid syphilis test. HIV test results were anonymous and unlinked because providing both pre- and post-test counselling to all study respondents was not possible in such an epidemiological survey. All respondents were, however, given referrals to the closest VCT centers. 


\section{Risks and benefits}

The benefits to the research participant included access to STI testing and to appropriate medical treatment based on the WHO syndromic management and treatment guidelines. Syndromic management has been shown to be effective in the management of people with genital ulcers (Moodley et al. 2003; Cheluget et al. 2004) and has been shown to work well in the management of men with urethritis in some settings (Wang et al. 2004). The study design also included an on-site rapid syphilis diagnosis, supplemented with confirmatory quantitative RPR and TPHA tests for those respondents who tested positive on the rapid tests. ${ }^{3}$ The advantage of this approach was that participants received immediate results and treatment, thereby minimizing the possibilities for missed treatment opportunities. The research participant also benefited from immediate access to the team doctor to discuss any other health problems. The respondent was also provided referral to qualified medical professionals and to the nearest VCT center.

The physical risks to the research participant included the physical discomfort of having blood taken. The study used the latest technology (vacutainer) to minimize the physical discomfort of having blood drawn. Careful steps to ensure infection prevention during sample taking were followed to minimize the physical risks.

It is central to the principles of good clinical care to ensure that participants received appropriate care for the conditions diagnosed in the study. In some cases, following the syndromic management protocol, men received the appropriate treatment at the time of participation; for others, the need for treatment only became known when the laboratory results were available. Therefore, each participant was asked how he could be contacted following the confirmation of biological results. In order to ensure that even those who participated at the end of the survey received their lab results, the doctors were employed for one month after data collection was completed. This ensured that all follow-up activities were completed.

All laboratory results were sent by SIUT to the Population Council office in Islamabad; they were then sent to the study team doctors in each city. Individual results were provided in a sealed envelope along with instructions for accessing the nearest VCT center in case the respondent wanted to know more about his test results or his HIV

\footnotetext{
${ }^{3}$ Such an approach was recommended by Dr. Rosanna Peeling in her review of the original design (in 2002) and was confirmed through recent (July 2005) email correspondence with Dr. Peeling.
} 
status. Upon production of his PIN number, the individual envelope containing the results was provided to the respondent by the study team doctor.

\section{Partner management}

Partner management (PM) is a cornerstone of good STI prevention and care policies; however, it is often overlooked or ineffectively implemented. There are few surveys evaluating what works in terms of partner management in a variety of geographical settings, and most trials of PM have taken place in the United States or Europe. However, our experience in South Asia had highlighted a number of approaches to partner management that were used in this study. The first was that clients prefer to take away additional drugs to give to their partners rather than refer their partners for care (unpublished results). Therefore, whenever feasible, the doctor on hand provided appropriate advice for partner management.

\section{Referral to higher levels of care}

Since we were working closely with medical colleges in all of the cities, we ensured that those who were judged to be in need of immediate care were 'fast-tracked' to the appropriate level of treatment. To the extent possible, we tried to ensure that participants were seen on the day of their visit and within a reasonable waiting time.

\section{Waste management}

All research staff members were trained in methods for proper handling and disposal of waste generated by the study. These included needle disposal through incineration, and all other materials associated with the biological testing (pipette tips, etc.) were cleansed in ten percent household bleach and thrown away. All other nonhazardous materials related to the study were carried off the premises and disposed of safely in hospitals.

\section{Compensation}

The study participants were not financially compensated for participating in the study. However, all participants had the option to discuss any general health or STI-related health problem with the study team doctor. Those participants diagnosed with an STI, based on self-reporting or through syndromic management guidelines, were treated free-of-charge by the study team doctor according to the WHO syndromic approach. 


\section{Study limitations}

Despite the careful measures employed to maintain the quality of the data several issues beyond the control of the study design came up that may be potential limitations of the study. These are described below.

\section{Recall bias}

It is well known that self-reported information, particularly related to sexual behavior, can be subject to recall bias (Cleland et al. 2004). Accurate recall is less likely when reporting on events that have occurred over a long period of time and for behaviors such as condom use, number of sexual partners, and frequency of sex. Therefore, this study focused on sexual behaviors in the past 12 months to minimize recall bias. The study also addressed this concern to some degree through rigorous training of the interviewers to remain nonjudgmental and objective. The fact that respondents had to undergo the biological testing also served as a crosscheck on self-reported behavior.

\section{Study design}

The study design was descriptive, analytical, and cross-sectional and therefore does not allow for determination of causality related to risk behaviors and exposure to specific interventions or to identify trends.

\section{Sampling and sample size}

Due to several administrative issues the commencement of the study was delayed many times. When fieldwork finally started, the timings for data collection were curtailed owing to Ramadan (the holy month of fasting). From the beginning, the researchers knew that it would be difficult to find men in their homes, especially those who were self-employed and did not follow any fixed work hours. From the pretest we learned that the most appropriate time to reach men would be in the evenings. The interviewers remained in the field for more than eight hours in order to reach men as they returned from work.

However, because of the delayed start to the study, the fieldwork had to be completed in only one month; as a result, the study teams were not able to spend more than one week in each PSU. Because of this limited time period and despite three visits to homes, some of the sampled respondents were not available. To minimize any 
potential sampling bias on replacement, we employed a systematic random replacement strategy.

\section{Timing issues}

Because of the delayed start, the study went into the field during the summer months when many of the students who would have been present in hostels were on leave as schools were closed. In addition there happened to be significant political unrest going on in the capital city, Islamabad. This affected the fieldwork in the neighboring city of Rawalpindi in terms of the availability of respondents.

\section{Response}

The target sample size for the study was 400 men in each of the six cities for a total sample size of 2,400. We anticipated relatively high rates of nonresponse due to the sensitive nature of the subject matter of the questionnaire, the biological testing requirement, and the known difficulty in locating men in urban settings (Mishra et al. 2006). In order to achieve our desired sample size, we employed a systematic replacement strategy of approaching the next house in the case of refusal, a locked house, or if a male respondent remained absent after three visits.

Out of the original 2,400 randomly sampled households, 1,338 (56 percent) were interviewed on primary visit and 1,062 (44 percent) were replaced because respondents were either ineligible, the dwelling premises were locked, respondents were absent after three follow-up visits, or the respondent refused.

The 1,062 interviews done through replacement were not unexpected. We were required to carry out several rounds of the replacement strategy in order to complete the sample size. Table 2.3 shows the number of replacement rounds required to achieve full replacement of the original sample size and the breakdown of households visited. Based on the original sampling and the systematic replacements, we touched a total of 5,995 households. 
Table 2.3: Number of survey responses

\begin{tabular}{lrrrr}
\hline Round & Dwellings visited & $\begin{array}{r}\text { Interviews completed } \\
\text { successfully }\end{array}$ & $\begin{array}{r}\text { Refusals } \\
\text { Religible or not found }\end{array}$ \\
\hline 1 & 2,400 & 1,338 & 254 & 808 \\
2 & 1,062 & 309 & 270 & 483 \\
3 & 753 & 186 & 262 & 305 \\
4 & 567 & 134 & 160 & 273 \\
5 & 433 & 129 & 140 & 164 \\
6 & 304 & 65 & 174 & 65 \\
7 & 239 & 108 & 119 & 12 \\
8 & 131 & 71 & 28 & 32 \\
9 & 60 & 25 & 28 & 7 \\
10 & 35 & 25 & 6 & 4 \\
11 & 10 & 9 & 1 & 0 \\
12 & 1 & 1 & 0 & 0 \\
Total & $\mathbf{5 , 9 9 5}$ & $\mathbf{2 , 4 0 0}$ & $\mathbf{1 , 4 4 2}$ & $\mathbf{2 , 1 5 3}$ \\
\hline
\end{tabular}

After employing our replacement strategy, we completed 2,400 full behavioral interviews. Out of the 2,400 who gave interviews, 2,396 (99.8 percent) participated in the biological testing.

To verify whether there was any significant difference between the original sampled households and the replacement households, statistical analysis comparing the prevalence of STIs between the two groups was carried out. This analysis showed no statistical difference in the STI prevalence within the two groups.

The refusal rate for our study was 37 percent $(1,442 / 3,856)$ which is well within the range of similar studies (Mishra et al. 2006; PDHS 1991). The main reason for refusal was due to issues related to the timing of the study. The majority of the respondents who were absent were in Rawalpindi, where during the survey period serious political disturbances were taking place in the neighboring city of Islamabad. In addition, the study was conducted during the summer months when some families had temporarily shifted to spend summer vacations with relatives.

Because of concerns that there might be something unique about the sociodemographic characteristics of the men who were absent, we revisited a random sample of those reported as absent in Rawalpindi after completion of the study during October and November 2007. During these follow-up visits, only 23 percent (123 out of 541) respondents were found to be present at home, with the remainder still absent 
after three visits, confirming our earlier results that these men were difficult to locate. The study team was able to collect basic sociodemographic information on the absent respondents from other family members in the household. These results suggest that those found absent were not substantially different from those who had been successfully replaced. 


\section{SOCIODEMOGRAPHIC CHARACTERISTICS OF RESPONDENTS}

This chapter describes individual socio demographic characteristics of those who participated in this study.

\section{Age}

The mean age of the respondents in all six cities was 29.1 years; the median age was 27.0 years (table 3.1). The largest age group was ages 21-25 years; the smallest group was 3135 years. More than 60 percent were ages 30 and under. The mean age for Faisalabad was 29.6 years; for Karachi - 27.0 years; Lahore 30.0 years; Peshawar - 28.0 years; Quetta 30.0 years; and for Rawalpindi it was 28.0 years. (For age of respondents by city, see appendix 1.)

\section{Education}

Overall 15 percent of the respondents had not received any education (table 3.2). Fifty-three percent had completed up to secondary schooling, which also includes religious education. Approximately 32 percent of the respondents had received education beyond matriculation.

The highest number of uneducated respondents was in Faisalabad (18 percent); the lowest was in Rawalpindi (7 percent). The highest proportion of master's degree
Table 3.1: Age of respondents (in years)

\begin{tabular}{lr}
\hline Age & Percent \\
\hline $16-20$ & 19.3 \\
$21-25$ & 23.8 \\
$26-30$ & 17.8 \\
$31-35$ & 11.8 \\
$36-40$ & 12.8 \\
$41-45$ & 14.5 \\
Total & $\mathbf{1 0 0 . 0}$ \\
\hline Mean age in years & 29.1 \\
Median age in years & 27.0 \\
\hline (N) & $\mathbf{( 2 , 4 0 0 )}$ \\
\hline
\end{tabular}

Table 3.2: Education of respondents

\begin{tabular}{lr}
\hline Education & Percent \\
\hline No education & 14.9 \\
Up to secondary & 53.2 \\
Above secondary & 31.9 \\
Total & 100.0 \\
(N) & $(\mathbf{2 , 4 0 0 )}$ \\
\hline
\end{tabular}


holders was in Rawalpindi. This corresponds to national statistics that show Rawalpindi to have the highest male literacy ratio compared to Faisalabad (81 and 60 percent, respectively) (PSLM 2004-2005). (For education of respondents by city, see appendix 1.)

\section{Employment}

Among all of the respondents 17 percent were enrolled as students and 3 percent were unemployed (table 3.3). The remaining 80 percent reported being employed.

Most respondents were employed in the private sector (39 percent) followed by selfemployed (32 percent) and government service (19 percent) (table 3.4). By city, the highest number of men employed in the private sector was in Faisalabad followed by Karachi (50 and 44 percent, respectively). The highest number self-employed was in Lahore (38 percent); Peshawar had the highest number of government employees (23 percent). (For employment status and details of respondents by city, see appendix 1 ).
Table 3.3: Employment of respondents

\begin{tabular}{lr}
\hline Employment & Percent \\
\hline Unemployed & 3.0 \\
Student & 16.8 \\
Employed & 80.3 \\
Total & 100.0 \\
(N) & $(\mathbf{2 , 4 0 0 )}$ \\
\hline
\end{tabular}

Table 3.4: Type of employment

\begin{tabular}{lr}
\hline Category & Percent \\
\hline Government service & 19.3 \\
Private service & 39.3 \\
Self-employed & 32.4 \\
Other & 8.9 \\
Total & $\mathbf{1 0 0 . 0}$ \\
(N) & $(\mathbf{1 , 9 2 3 )}$ \\
\hline
\end{tabular}

\section{Marital status}

Overall, 48 percent of the respondents were unmarried and 52 percent were married; less than 1 percent reported being formerly married (table 3.5).

As figure 3.1 shows, the fewest married men (3 percent) were in the age group 16-20 years.
Table 3.5: Marital status of respondents

\begin{tabular}{lr}
\hline Marital status & Percent \\
\hline Unmarried & 47.6 \\
Married & 51.8 \\
Formerly married & 0.7 \\
Total & $\mathbf{1 0 0 . 0}$ \\
(N) & $(\mathbf{2 , 4 0 0 )}$ \\
\hline
\end{tabular}
Twenty percent of married men were in the age range of 21-25 years, while 58 percent of respondents in the age range of 26-30 years were married. Almost all men in the category of $41-45$ years were married. The results of marital status by age show that the 
number of married men increases correspondingly with age. The mean age of married men was 35 years. (For marital status of respondents by city, see appendix 1.)

Figure 3.1: Percentage of married men, within age groups

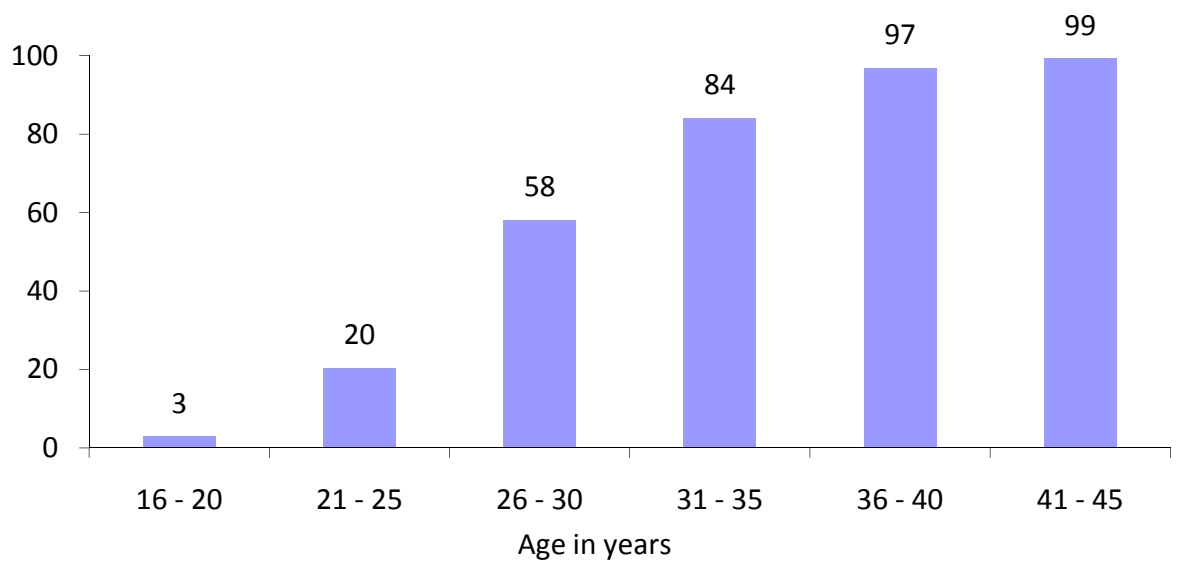

\section{Language}

The most common language was Punjabi (44 Table 3.6: Respondents' languages percent), followed by Pashto (24 percent) and Language Percent Urdu (14 percent) (table 3.6). Karachi was the Punjabi 43.8 most multi-ethnic city where all major Pakistani Pashto 23.8 languages were reported. One-third of respondents living in Karachi reported Urdu as Other $^{\mathrm{a}}$ their mother tongue. Punjabi was the only $\frac{(\mathbf{N})}{(2,400)}$ language to be spoken in all six cities of the Brahvi, Hindko, and Farsi. country. The highest number of Punjabi speakers was reported in Faisalabad (90 percent), followed by Lahore (78 percent) and Rawalpindi (59 percent). (For language of respondents, see appendix 1).

\section{Economic status}

Thirty-nine percent of all respondents were within the lowest monthly earning income group of Rs. 15,000; 50 percent reported earning Rs. 5-15,000 (table 3.7). Eleven percent of respondents reported monthly earnings of over Rs. 15,000. (For economic status of respondents by city, see appendix 1.)
Table 3.7: Self-reported monthly income (in Pakistani rupees ${ }^{a}$ )

Self-reported monthly

\begin{tabular}{lr} 
income & Percent \\
\hline $1,000-5,000$ & 39.2 \\
$5,001-15,000$ & 50.1 \\
15,001 and above & 10.7 \\
Total & $\mathbf{1 0 0 . 0}$ \\
(N) & $\mathbf{( 2 , 4 0 0 )}$ \\
\hline a &
\end{tabular}

${ }^{a}$ At time of study: US\$1 = 60 Pakistani rupees. 


\section{Household possessions}

In all six cities, 98 percent of the respondents had electricity connections in their households, 82 percent owned a television set, and 71 percent had a refrigerator. Eighteen percent of the respondents had a car while 37 percent reported owning a motorcycle. The highest television set ownership was reported in Rawalpindi and Lahore (89 percent each). The lowest television ownership was in Karachi (70 percent) where there was a reportedly high proportion of migrant men living alone or with relatives.

\section{Living arrangements and migrant status}

Within the total sample, 43 percent of the respondents were residing with their parents; almost all of these men were unmarried. Twenty-two percent were residing with their parents and wives and 23 percent were living with their wives. Twelve percent were either living alone or with friends or other relatives. Out of those living
Table 3.8: Living arrangements of respondents

\begin{tabular}{lr}
\hline Person(s) living with & Percent \\
\hline Parents & 43.1 \\
Parents and wife & 21.8 \\
Wife & 22.8 \\
Others & 12.3 \\
Total & $\mathbf{1 0 0 . 0}$ \\
(N) & $\mathbf{( 2 , 4 0 0 )}$ \\
\hline
\end{tabular}

alone, 67 percent were unmarried. (For living arrangements and migrant status of respondents by city, see appendix 1.)

We determined migrant status by asking the respondent if he had currently been living away from his marital or natal home for more than four months. Based on this definition, the overall population of migrants in the six cities was less than 9 percent (figure 3.2). The highest numbers of migrants were reported in Karachi and Rawalpindi (24 percent and 23 percent, respectively). Karachi is a well-known cosmopolitan port city attracting migrants from all over the country. Rawalpindi, being adjacent to the federal capital, attracts workers from other cities to work in Islamabad. Quetta (4 percent), Faisalabad (4 percent), and Lahore (3 percent) had the lowest numbers of migrants.

We also asked respondents about the number of overnight stays outside their home per month for occupational reasons. If a respondent reported spending more than one night away from home for occupational reasons we classified him as a 'traveler.' In the 
study population, 16 percent of the respondents were travelers. As figure 3.2 shows, in all cities, except Rawalpindi, there were consistently more travelers than migrants. The categories of 'migrant' and 'traveler' were not mutually exclusive.

Figure 3.2: Migration status of respondents, by city

Migration status $(95 \% \mathrm{Cl})$

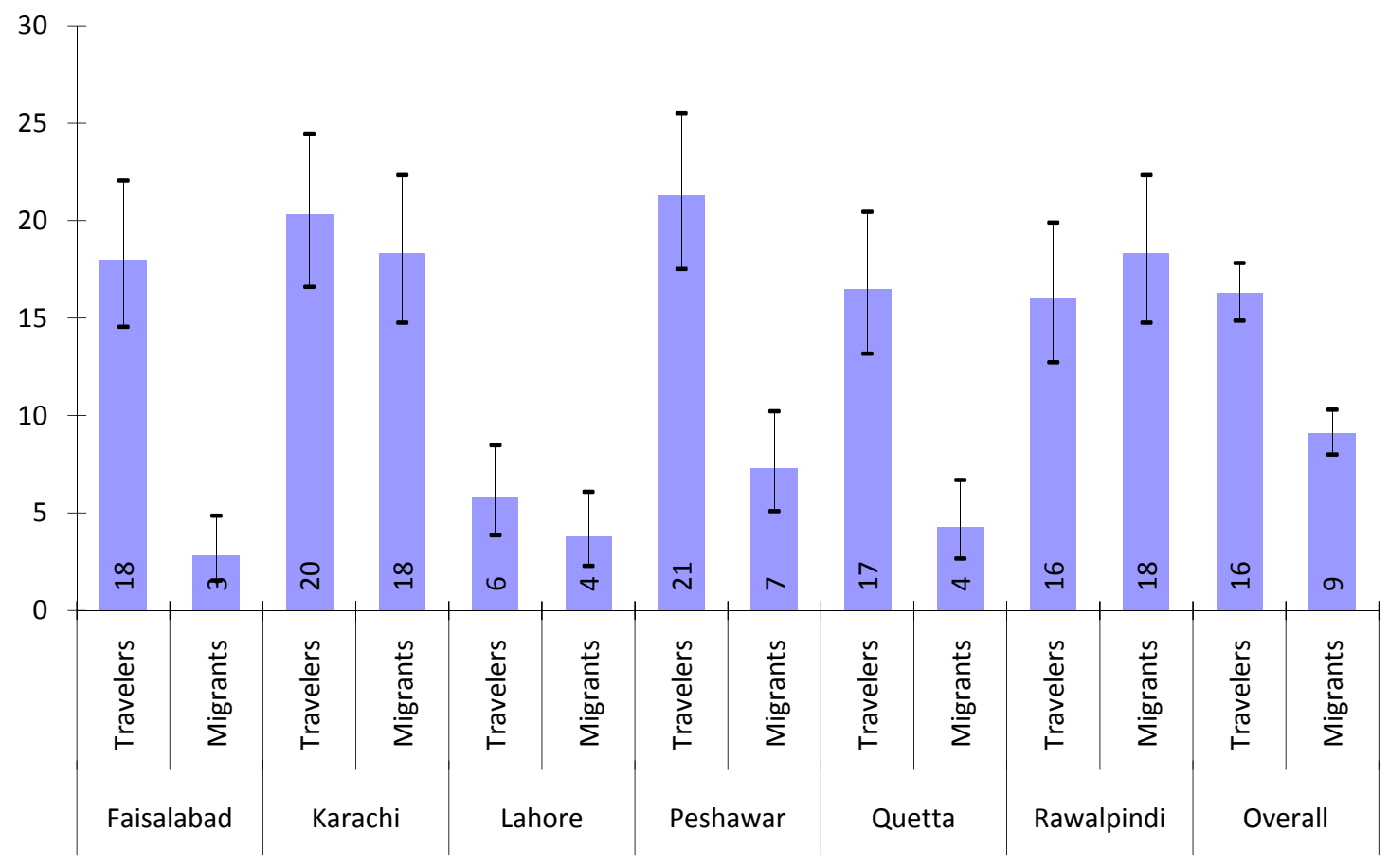





\section{AWARENESS AND KNOWLEDGE ABOUT HIV/AIDS AND OTHER STIS}

International research evidence clearly demonstrates that any attempt to modify behaviors requires first determining existing levels of knowledge and attitudes (Amon et al. 2000). To evaluate or measure the impact of any STI-related information, education and communication (IEC) activity, evidence about people's knowledge of the modes of transmission of sexually transmitted infections (including HIV/AIDS), and their beliefs and misconceptions about protective behaviors, is required. Examining differences in knowledge and beliefs by socioeconomic group, geographical area, and educational level is also important for the design of IEC activities. This section presents the respondents' self-reported knowledge of sexually transmitted infections (STIs) including HIV/AIDS; their understandings of protection against STIs; and their perceived risk of acquiring an STI.

\section{Awareness/knowledge of HIV/AIDS}

\section{Awareness}

Out of the total number of respondents, almost all (90 percent) had heard of the term "HIV/AIDS" (figure 4.1). The highest number of respondents who had heard of the term HIV/AIDS was in Rawalpindi (98 percent), followed by Quetta (97 percent), Peshawar (95 percent), Faisalabad (93 percent), and Lahore (93 percent). In Karachi, less than three-quarters of the men had heard of the term HIV/AIDS (70 percent). The 95 percent confidence intervals (CI) are also shown in figure 4.1. 
Figure 4.1: Percentage of respondents who had heard the term HIV/AIDS (95\% CI), by city

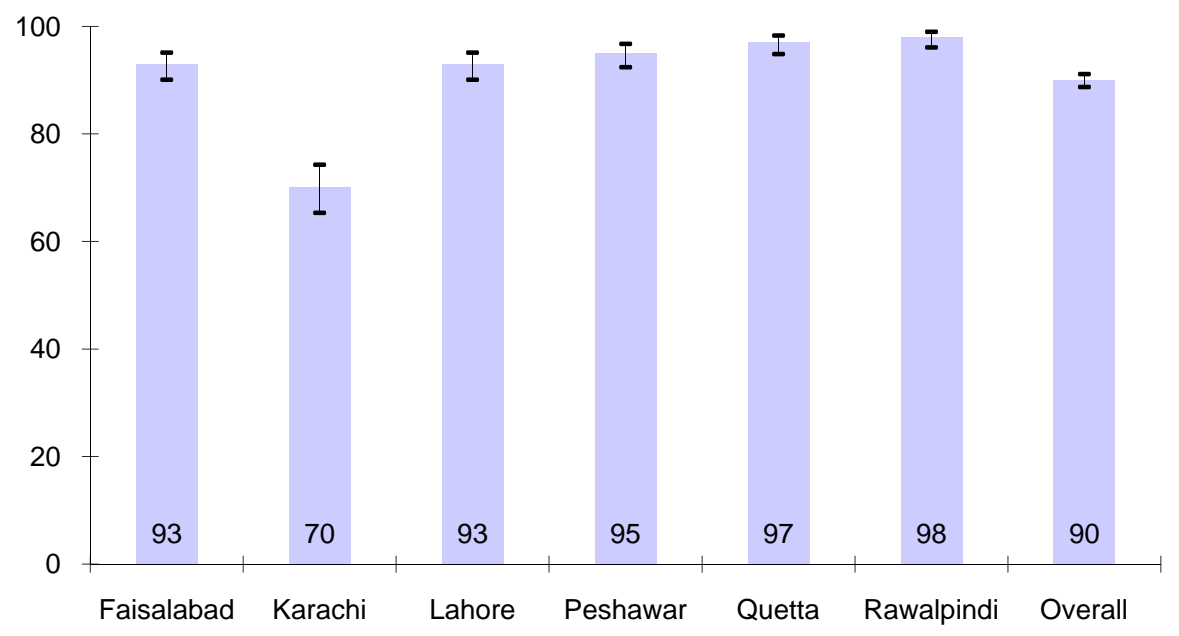

\section{Source of knowledge of HIV/AIDS}

As table 4.1 shows, television was the main source of knowledge about HIV/AIDS with 84 percent reporting it as a source. Other sources were friend/family member (30 percent); books/newspapers (24 percent); health providers (19 percent); and radio (15 percent).

Table 4.1: Percentage distribution of respondents according to source of information regarding HIV/AIDS, by city

\begin{tabular}{lrrrrrrr}
\hline Source & Faisalabad & Karachi & Lahore & Peshawar & Quetta & Rawalpindi & Overall \\
\hline Health provider & 16.8 & 28.8 & 8.9 & 14.7 & 21.4 & 23.5 & $\mathbf{1 8 . 5}$ \\
TV & 85.9 & 69.1 & 85.0 & 84.5 & 81.7 & 91.4 & $\mathbf{8 3 . 9}$ \\
Radio & 6.1 & 9.1 & 14.4 & 14.2 & 17.6 & 28.1 & $\mathbf{1 5 . 4}$ \\
Book/newspaper & 29.6 & 19.3 & 11.3 & 35.2 & 25.1 & 23.8 & $\mathbf{2 4 . 3}$ \\
Friend/family member & 38.9 & 30.0 & 21.5 & 38.8 & 28.2 & 21.3 & $\mathbf{2 9 . 7}$ \\
Other & 0.8 & 2.9 & 1.3 & 2.4 & 2.3 & 1.0 & $\mathbf{1 . 7}$ \\
Cannot recall & 0.8 & 0.8 & 0.8 & 0.8 & 0.0 & 0.5 & $\mathbf{0 . 6}$ \\
(N) & $\mathbf{( 3 7 5 )}$ & $\mathbf{( 2 4 3 )}$ & $\mathbf{( 3 8 2 )}$ & $\mathbf{( 3 8 4 )}$ & $\mathbf{( 3 8 9 )}$ & $\mathbf{( 3 9 6 )}$ & $\mathbf{( 2 , 1 6 9 )}$ \\
\hline
\end{tabular}

Note: Multiple responses were possible; columns do not total 100 percent.

The focus group discussions also support this finding, as the majority of the FGD respondents mentioned television and newspaper advertisements as their main sources of information about STIs and HIV/AIDS. In the FGDs, some respondents also mentioned banners/posters displayed by the government and campaigns launched by NGOs. 
Examining the level of HIV knowledge by educational status suggests a statistically significant difference. Of the 240 respondents who had not heard of the term "HIV/AIDS" (10 percent), almost two-thirds (61 percent) had not received any education. The difference between educated and uneducated men with regards to $\mathrm{HIV} / \mathrm{AIDS}$ awareness in all cities was statistically significant ( $\mathrm{p}$-value $<0.05$ ).

In response to the question whether a healthy looking man can be infected with HIV, the highest number of respondents who answered correctly ('yes') were in Rawalpindi (77 percent), followed by Faisalabad (73 percent), Peshawar (72 percent), and Lahore (69 percent). The lowest proportion of respondents (40 percent) who provided the correct answer was in Karachi. The poor response in Karachi could be attributed to the large migrant population there. Owing to the temporary nature of their stay, migrants do not have access to basic amenities or television, which was reported to be a major source of information.

\section{Modes of transmission of HIV/AIDS}

Those respondents who reported having heard of the word HIV/AIDS $(n=2,169)$ were asked about the modes of HIV transmission and the methods a person can adopt to protect himself against acquiring the infection. Multiple responses were allowed for each question. Respondents were first given an opportunity to provide unprompted responses; they were asked subsequently whether or not they agreed with a list of prepared responses. The list of responses included five routes of HIV transmission: (1) unprotected sex; (2) injections; (3) infected blood; (4) needles and shaving objects; and (5) breastfeeding.

Unprompted spontaneous answers are generally considered a better measure of a respondent's knowledge as compared to prompted responses (Family Health International 2000). Prompted responses can be influenced by the respondent's desire to conform to what he considers to be the right response. The following tables present both prompted and unprompted responses.

Tables 4.2 and 4.3 present knowledge levels about modes of HIV transmission overall and by city. Overall, there was knowledge regarding three common modes of transmission (sexual route, infected blood, and unclean injection needles). Eighty-five percent of respondents mentioned the sexual transmission route without prompting; 49 percent mentioned unclean syringes. Few men knew that needles or sharp objects could transmit HIV/AIDS (34 percent across all cities; 24 percent in Quetta; 22 percent 
in Karachi). This increased to 57 percent with prompting, but the unprompted results are a concern given that this mode of transmission is very relevant in the Pakistani context. Less than 5 percent of respondents in each city knew that HIV/AIDS could be transmitted from a mother to her infant through breastfeeding. This percentage increased to 55 percent with prompting. So, despite the high rate of recognition of the term HIV/AIDS, the data show that levels of knowledge about specific modes of transmission are low.

Table 4.2: Percentage distribution of respondents who had heard the term HIV/AIDS, according to knowledge about common modes of HIV transmission, by city

\begin{tabular}{lrrrrrrr}
\hline $\begin{array}{l}\text { Mode of transmission } \\
\text { and knowledge }\end{array}$ & Faisalabad & Karachi & Lahore & Peshawar & Quetta & Rawalpindi & Overall \\
\hline Sexual contact & & & & & & & \\
Yes unprompted & 80.5 & 75.7 & 80.6 & 89.1 & 88.9 & 89.9 & $\mathbf{8 4 . 7}$ \\
Yes prompted & 18.7 & 19.8 & 17.3 & 9.4 & 8.5 & 9.3 & $\mathbf{1 3 . 4}$ \\
No & 0.3 & 0.4 & 0.0 & 0.5 & 0.3 & 0.0 & $\mathbf{0 . 2}$ \\
Don't know & 0.5 & 4.1 & 2.1 & 1.0 & 2.3 & 0.8 & $\mathbf{1 . 7}$ \\
Needle or sharp objects & & & & & & & \\
Yes unprompted & 36.0 & 21.8 & 37.2 & 41.1 & 23.9 & 40.2 & $\mathbf{3 4 . 1}$ \\
Yes prompted & 59.7 & 54.7 & 55.5 & 56.5 & 61.4 & 54.0 & $\mathbf{5 7 . 1}$ \\
No & 1.6 & 5.3 & 3.4 & 1.0 & 6.2 & 1.8 & $\mathbf{3 . 1}$ \\
Don't know & 2.7 & 18.1 & 3.9 & 1.3 & 8.5 & 4.0 & $\mathbf{5 . 7}$ \\
Unclean injection needle/syringe & & & & & & & \\
Yes unprompted & 40.3 & 41.2 & 46.9 & 49.5 & 52.7 & 62.1 & $\mathbf{4 9 . 4}$ \\
Yes prompted & 58.7 & 52.7 & 50.3 & 49.0 & 45.0 & 36.4 & $\mathbf{4 8 . 3}$ \\
No & 0.3 & 1.2 & 0.8 & 0.5 & 0.3 & 0.0 & $\mathbf{0 . 5}$ \\
Don't know & 0.8 & 4.9 & 2.1 & 1.0 & 2.1 & 1.5 & $\mathbf{1 . 9}$ \\
Total & $\mathbf{1 0 0 . 0}$ & $\mathbf{1 0 0 . 0}$ & $\mathbf{1 0 0 . 0}$ & $\mathbf{1 0 0 . 0}$ & $\mathbf{1 0 0 . 0}$ & $\mathbf{1 0 0 . 0}$ & $\mathbf{1 0 0 . 0}$ \\
(N) & $\mathbf{( 3 7 5 )}$ & $\mathbf{( 2 4 3 )}$ & $\mathbf{( 3 8 2 )}$ & $\mathbf{( 3 8 4 )}$ & $(\mathbf{3 8 9}$ & $\mathbf{( 3 9 6 )}$ & $\mathbf{( 2 , 1 6 9 )}$ \\
\hline
\end{tabular}


Table 4.3: Percentage distribution of respondents who had heard the term HIV/AIDS according to knowledge about other modes of HIV/AIDS transmission, by city

Mode of transmission

and knowledge Faisalabad Karachi Lahore Peshawar Quetta Rawalpindi Overall

Transfusion of infected blood

\begin{tabular}{lrrrrrrr} 
Yes unprompted & 34.4 & 30.0 & 50.0 & 40.9 & 49.9 & 70.7 & $\mathbf{4 7 . 2}$ \\
Yes prompted & 63.5 & 53.9 & 47.1 & 57.6 & 46.5 & 28.0 & $\mathbf{4 9 . 0}$ \\
No & 0.5 & 1.2 & 0.8 & 0.0 & 0.3 & 0.0 & $\mathbf{0 . 4}$ \\
Don't know & 1.6 & 14.8 & 2.1 & 1.6 & 3.3 & 1.3 & $\mathbf{3 . 4}$ \\
Mother to infant through breastfeeding & & & & & & \\
Yes unprompted & 2.4 & 4.5 & 3.1 & 4.2 & 2.8 & 4.8 & $\mathbf{3 . 6}$ \\
Yes prompted & 57.9 & 48.6 & 58.1 & 61.2 & 39.6 & 62.4 & $\mathbf{5 5 . 0}$ \\
No & 10.7 & 11.9 & 16.2 & 14.8 & 26.5 & 19.7 & $\mathbf{1 7 . 0}$ \\
Don't know & 29.1 & 35.0 & 22.5 & 19.8 & 31.1 & 13.1 & $\mathbf{2 4 . 4}$ \\
Total & $\mathbf{1 0 0 . 0}$ & $\mathbf{1 0 0 . 0}$ & $\mathbf{1 0 0 . 0}$ & $\mathbf{1 0 0 . 0}$ & $\mathbf{1 0 0 . 0}$ & $\mathbf{1 0 0 . 0}$ & $\mathbf{1 0 0 . 0}$ \\
(N) & $\mathbf{( 3 7 5 )}$ & $\mathbf{( 2 4 3 )}$ & $\mathbf{( 3 8 2 )}$ & $\mathbf{( 3 8 4 )}$ & $\mathbf{( 3 8 9 )}$ & $\mathbf{( 3 9 6 )}$ & $\mathbf{( 2 , 1 6 9 )}$ \\
\hline
\end{tabular}

As mentioned earlier, respondents were asked to name transmission routes for HIV/AIDS. To determine their level of knowledge, the five possible responses obtained after prompting were converted into a three-category Likert scale. Respondents who provided no response even after prompting were categorized as having 'no' knowledge; those who provided one to three correct responses were categorized as having 'medium' knowledge; and those who provided more than three correct responses were classified as having 'high' knowledge. Note that these categories are used in figures 4.2-4.4. Rawalpindi had the highest level of knowledge (95 percent), followed by Peshawar (92 percent), Quetta (84 percent), Faisalabad (90 percent), and Lahore (89 percent). Karachi had the lowest level of knowledge, with a sizeable proportion of respondents (41 percent), providing no response, even after prompting. (This information is shown in figure 4.2.)

Figure 4.3 shows that those respondents who owned a television had higher levels of knowledge about HIV. Figure 4.4 shows that levels of HIV knowledge are also associated with socioeconomic status. The assumption is that those earning a higher salary probably have greater exposure to the electronic and print media, which carry health education messages. The high level of knowledge of the unemployed respondents is likely explained by the inclusion of students in this category. 
Figure 4.2: Percentage distribution of respondents according to level of knowledge of HIV transmission (after prompting), by city

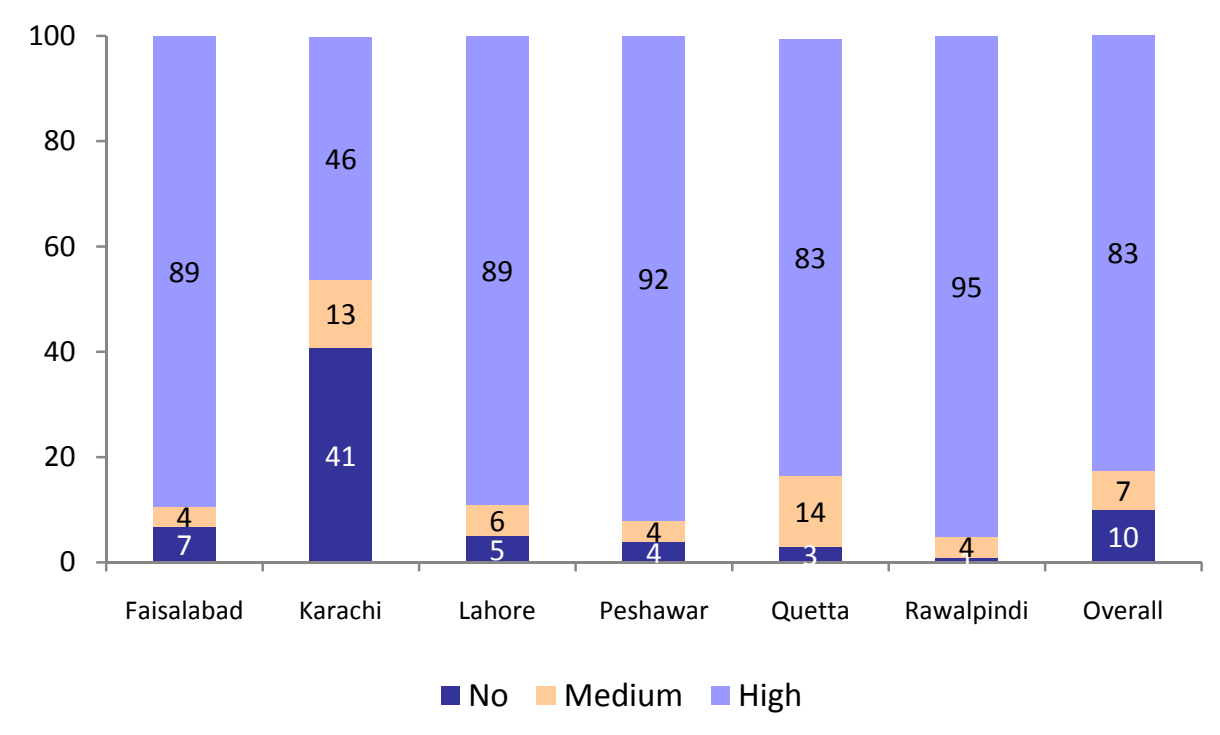

Figure 4.3: Level of HIV knowledge and TV ownership

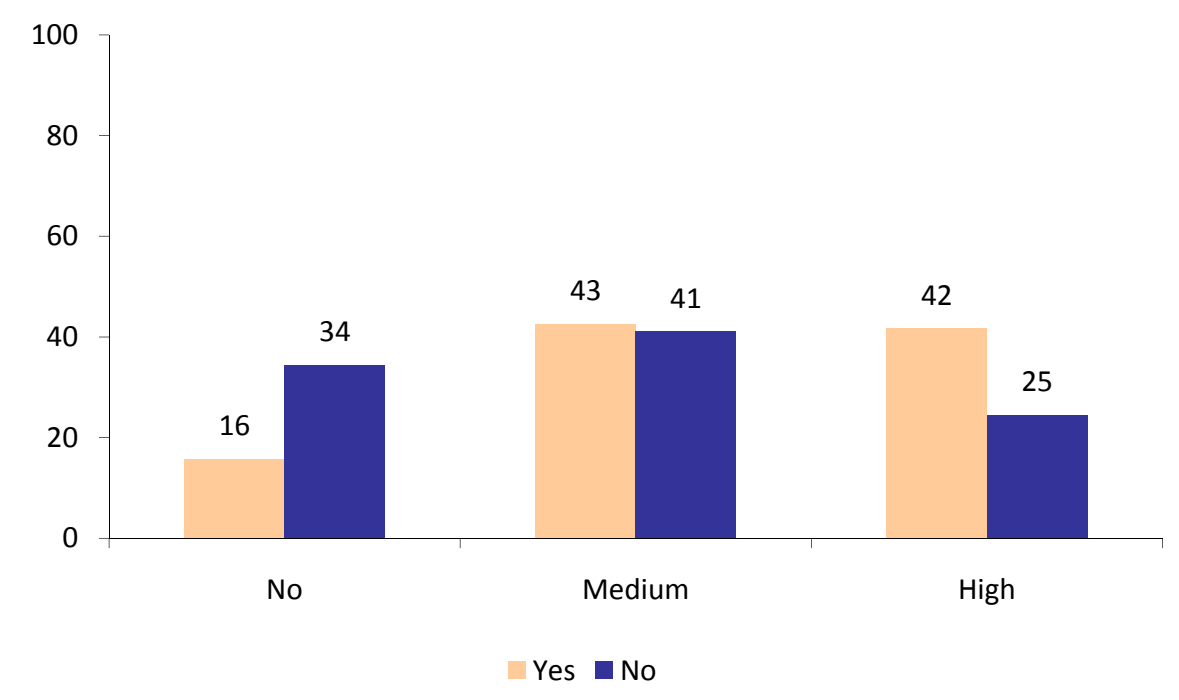


Figure 4.4: Level of HIV knowledge and socioeconomic status

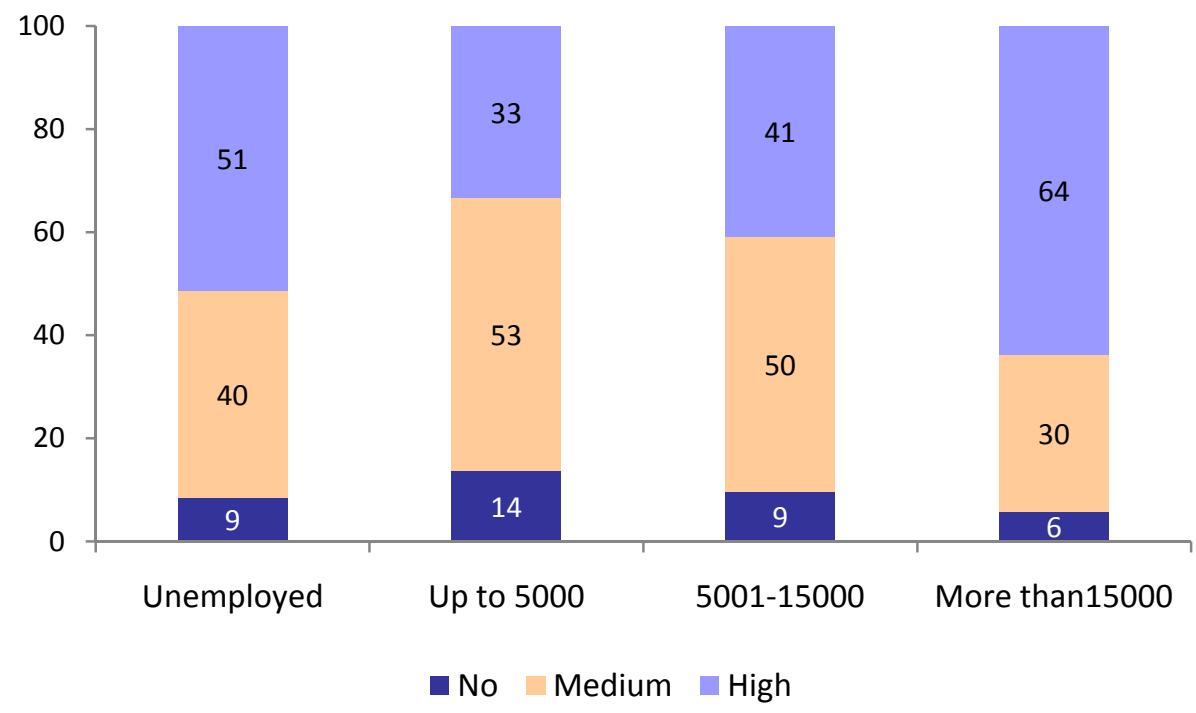

Myths and misconceptions regarding transmission. Table 4.4 describes some of the myths and misconceptions about HIV/AIDS transmission among the study respondents. Nearly half of all respondents had misconceptions regarding the spread of HIV infection; nearly one-quarter of the respondents mentioned that HIV infection could be spread through the sharing of food (26 percent), clothing ( 24 percent), bedding ( 26 percent), and toilets ( 24 percent) with an infected person. Sixteen percent mentioned that shaking hands with an infected person could lead to transmission. If we combine the incorrect and don't know responses, nearly 50 percent of the respondents had misconceptions regarding the mode of transmission. Respondents in Peshawar, Quetta, and Rawalpindi seemed to report more accurately about transmission modes.

The focus group discussions also showed that men's perceptions about sexually transmitted infections are somewhat vague and unclear (see appendix 3 for presentation of the qualitative data results). 
Table 4.4: Percentage distribution of respondents who had heard of HIV/AIDS, according to myths and misconceptions about HIV/AIDS, by city

\begin{tabular}{lrrrrrrr}
\hline Mode of HIV transmission & Faisalabad & Karachi & Lahore & Peshawar & Quetta & Rawalpindi & Overall \\
\hline Sharing food with an infected person & & & & & & \\
Yes & 39.5 & 30.9 & 24.1 & 24.2 & 16.7 & 20.2 & $\mathbf{2 5 . 5}$ \\
No & 35.7 & 33.7 & 47.1 & 62.2 & 65.6 & 67.7 & $\mathbf{5 3 . 4}$ \\
Don't know & 24.8 & 35.4 & 28.8 & 13.5 & 17.7 & 12.1 & $\mathbf{2 1 . 1}$ \\
Sharing clothes with an infected person & & & & & & \\
Yes & 37.9 & 29.6 & 22.0 & 24.7 & 15.7 & 17.7 & $\mathbf{2 4 . 2}$ \\
No & 36.5 & 35.4 & 47.6 & 59.9 & 63.8 & 65.7 & $\mathbf{5 2 . 7}$ \\
Don't know & 25.6 & 35.0 & 30.4 & 15.4 & 20.6 & 16.7 & $\mathbf{2 3 . 1}$ \\
Sharing bedding with an infected person & & & & & & \\
Yes & 37.6 & 30.5 & 24.1 & 25.5 & 18.0 & 21.0 & $\mathbf{2 5 . 7}$ \\
No & 36.3 & 32.5 & 45.8 & 60.7 & 62.5 & 63.6 & $\mathbf{5 1 . 5}$ \\
Don't know & 26.1 & 37.0 & 30.1 & 13.8 & 19.5 & 15.4 & $\mathbf{2 2 . 7}$ \\
Mosquito/insect bite & & & & & & & \\
Yes & 44.3 & 27.2 & 34.0 & 50.3 & 21.9 & 38.6 & $\mathbf{3 6 . 6}$ \\
No & 19.5 & 32.1 & 34.6 & 28.1 & 47.6 & 39.1 & $\mathbf{3 3 . 7}$ \\
Don't know & 36.3 & 40.7 & 31.4 & 21.6 & 30.6 & 22.2 & $\mathbf{2 9 . 7}$ \\
Shaking hands with an infected person & & & & & & \\
Yes & 21.9 & 18.5 & 17.8 & 15.1 & 8.5 & 15.9 & $\mathbf{1 6 . 1}$ \\
No & 50.7 & 46.1 & 52.1 & 73.7 & 76.3 & 71.5 & $\mathbf{6 2 . 9}$ \\
Don't know & 27.5 & 35.4 & 30.1 & 11.2 & 15.2 & 12.6 & $\mathbf{2 1 . 0}$ \\
Sharing toilet with an infected person & & & & & & \\
Yes & 30.9 & 26.3 & 21.2 & 24.2 & 24.4 & 18.4 & $\mathbf{2 4 . 1}$ \\
No & 39.5 & 35.0 & 44.8 & 52.6 & 48.3 & 61.1 & $\mathbf{4 7 . 8}$ \\
Don't know & 29.6 & 38.7 & 34.0 & 23.2 & 27.2 & 20.5 & $\mathbf{2 8 . 2}$ \\
Total & $\mathbf{1 0 0 . 0}$ & $\mathbf{1 0 0 . 0}$ & $\mathbf{1 0 0 . 0}$ & $\mathbf{1 0 0 . 0}$ & $\mathbf{1 0 0 . 0}$ & $\mathbf{1 0 0 . 0}$ & $\mathbf{1 0 0 . 0}$ \\
(N) & $\mathbf{3 7 5 )}$ & $\mathbf{( 2 4 3 )}$ & $\mathbf{( 3 8 2 )}$ & $\mathbf{( 3 8 4 )}$ & $\mathbf{( 3 8 9 )}$ & $\mathbf{( 3 9 6 )}$ & $\mathbf{( 2 , 1 6 9 )}$ \\
\hline Note "Yes"
\end{tabular}

Note: "Yes" includes both prompted and unprompted responses.

\section{Perception of risk of HIV and HIV testing}

One measure of the perception of risk of HIV is whether or not the respondent has had his blood tested for HIV (Stein and Nyamathi 2000). Overall, only 9 percent of the respondents had been tested for HIV $(n=199)$ (table 4.5). The highest percentage who had their blood tested for HIV was in Quetta (18 percent), followed by Peshawar (13 percent), Rawalpindi (7 percent), Faisalabad (8 percent), Karachi (5 percent), and Lahore (5 percent). 
Table 4.5: Percentage distribution of men who had their blood tested for HIV/AIDS, by city

\begin{tabular}{lrrrrrrr}
\hline Variable & Faisalabad & Karachi & Lahore & Peshawar & Quetta & Rawalpindi & Overall \\
\hline Tested & 7.8 & 4.5 & 5.0 & 12.8 & 18.0 & 7.0 & $\mathbf{9 . 2}$ \\
Not tested & 92.3 & 95.5 & 95.0 & 87.3 & 82.0 & 93.0 & $\mathbf{9 0 . 8}$ \\
(N) & $\mathbf{( 4 0 0 )}$ & $\mathbf{( 4 0 0 )}$ & $\mathbf{( 4 0 0 )}$ & $\mathbf{( 4 0 0 )}$ & $\mathbf{( 4 0 0 )}$ & $\mathbf{( 4 0 0 )}$ & $\mathbf{( 2 , 4 0 0 )}$ \\
\hline
\end{tabular}

Overall, 41 percent of the men who had their blood tested for HIV/AIDS had it tested in government facilities (both government clinics and blood banks) and 48 percent in private health facilities (both private health clinics and blood banks) (table 4.6). When analyzed by city, more men in Peshawar, Faisalabad, Karachi, and Rawalpindi were tested at private health facilities as compared to government health facilities. In Lahore and Quetta more men were tested in a government health facility.

Although voluntary counseling and testing for HIV can be an important strategy to reduce risky behaviors and improve health-seeking behaviors, this approach is not common in Pakistan as there are only a handful of VCT centers in the country. Only two respondents in Karachi and one in Quetta specifically reported having had their blood tested for HIV at a VCT center.

Across all cities the main reasons for having an HIV/AIDS test was as part of the blood donation process (32 percent) or as a job requirement (27 percent), as well as self testing (22 percent) (table 4.7).

Table 4.6: Location of HIV/AIDS test, by city

\begin{tabular}{|c|c|c|c|c|c|c|c|c|c|c|c|c|c|c|}
\hline \multirow[b]{2}{*}{ Location } & \multicolumn{2}{|c|}{ Faisalabad } & \multicolumn{2}{|c|}{ Karachi } & \multicolumn{2}{|c|}{ Lahore } & \multicolumn{2}{|c|}{ Peshawar } & \multicolumn{2}{|c|}{ Quetta } & \multicolumn{2}{|c|}{ Rawalpindi } & \multicolumn{2}{|c|}{ Overall } \\
\hline & $\%$ & $\mathbf{N}$ & $\%$ & $\mathbf{N}$ & $\%$ & $\mathbf{N}$ & $\%$ & $\mathbf{N}$ & $\%$ & $\mathbf{N}$ & $\%$ & $\mathbf{N}$ & $\%$ & $\mathbf{N}$ \\
\hline $\begin{array}{l}\text { Government } \\
\text { facility }\end{array}$ & 32.2 & 10 & 38.9 & 7 & 40.0 & 8 & 33.4 & 17 & 52.8 & 38 & 35.7 & 10 & 40.9 & 90 \\
\hline Private facility & 48.4 & 15 & 50.0 & 9 & 25.0 & 5 & 58.8 & 30 & 45.9 & 33 & 50.0 & 14 & 48.2 & 106 \\
\hline VCT center & 0.0 & 0 & 11.1 & 2 & 0.0 & 0 & 0.0 & 0 & 1.4 & 1 & 0.0 & 0 & 1.4 & 3 \\
\hline No information & 19.4 & 6 & 0.0 & 0 & 35.0 & 7 & 7.8 & 4 & 0.0 & 0 & 14.3 & 4 & 9.5 & 21 \\
\hline Total & 100.0 & 31 & 100.0 & 18 & 100.0 & 20 & 100.0 & 51 & 100.0 & 72 & 100.0 & 28 & 100.0 & 220 \\
\hline
\end{tabular}


Table 4.7: Reasons for having an HIV/AIDS test, by city

\begin{tabular}{|c|c|c|c|c|c|c|c|c|c|c|c|c|c|c|}
\hline \multirow[b]{2}{*}{ Reason } & \multicolumn{2}{|c|}{ Faisalabad } & \multicolumn{2}{|c|}{ Karachi } & \multicolumn{4}{|c|}{ Lahore Peshawar } & \multicolumn{4}{|c|}{ Quetta Rawalpindi } & \multicolumn{2}{|c|}{ Overall } \\
\hline & $\%$ & $\mathbf{N}$ & $\%$ & $\mathbf{N}$ & $\%$ & $\mathbf{N}$ & $\%$ & $\mathbf{N}$ & $\%$ & $\mathbf{N}$ & $\%$ & $\mathbf{N}$ & $\%$ & $\mathbf{N}$ \\
\hline Recommended by doctor & 12.9 & 4 & 5.6 & 1 & 15.0 & 3 & 35.3 & 18 & 6.9 & 5 & 25.0 & 7 & 17.3 & 38 \\
\hline Self tested & 25.8 & 8 & 27.8 & 5 & 15.0 & 3 & 25.5 & 13 & 18.1 & 13 & 21.4 & 6 & 21.8 & 48 \\
\hline Job requirement & 25.8 & 8 & 33.3 & 6 & 50.0 & 10 & 19.6 & 10 & 20.8 & 15 & 39.3 & 11 & 27.3 & 60 \\
\hline Insurance & 0.0 & 0 & 16.7 & 3 & 0.0 & 0 & 0.0 & 0 & 1.4 & 1 & 0.0 & 0 & 1.8 & 4 \\
\hline For blood donation & 35.5 & 11 & 16.7 & 3 & 20.0 & 4 & 19.6 & 10 & 52.8 & 38 & 14.3 & 4 & 31.8 & 70 \\
\hline Total & 100.0 & 31 & 100.0 & 18 & 100.0 & 20 & 100.0 & 51 & 100.0 & 72 & 100.0 & & 100.0 & 220 \\
\hline
\end{tabular}

The respondents who reported having had an HIV/AIDS test were analyzed according to whether they had traveled to a foreign country. Of those who did report testing, 75 percent $(n=145)$ had not traveled abroad. The exception was Quetta, where 28 percent $(n=27)$ had traveled abroad.

\section{Protective measures against HIV/AIDS and other STls}

Respondents were asked to mention, unprompted, ways to prevent acquiring HIV/AIDS. Subsequently, prepared statements were read out and respondents were asked whether they agreed or disagreed with these statements, or did not know the answer. Overall, 18 percent of respondents knew, without being prompted, that a person can protect himself from getting HIV/AIDS by using a condom (table 4.8). When prompted, a further 47 percent said that using a condom offers protection, leaving 35 percent (more than one-third of the men) who responded either that condoms offer no protection against HIV/ AIDS or that they did not know the answer. The highest number of spontaneous correct responses related to condom use as a method of protection against HIV/AIDS was in Rawalpindi (29 percent) and Lahore (18 percent). In Karachi, 17 percent of the respondents spontaneously mentioned condom use as a protective measure; this increased to 48 percent with prompting.

The majority of respondents (84 percent) mentioned, without prompting, that a person can protect himself against acquiring an STI through sexual abstinence. The next highest unprompted response was using new needles (42 percent), followed closely by avoiding sex with sex workers (37 percent), and being monogamous by restricting sexual activity to one partner (27 percent). 
Table 4.8: Percentage distribution of respondents who knew about HIV/AIDS, according to knowledge of modes of protection against STIs (including HIV/AIDS), by city

\begin{tabular}{|c|c|c|c|c|c|c|c|}
\hline Mode of protection & Faisalabad & Karachi & Lahore & Peshawar & Quetta & Rawalpindi & Overall \\
\hline \multicolumn{8}{|l|}{ Abstaining from sex } \\
\hline Yes unprompted & 83.1 & 75.3 & 81.9 & 84.6 & 86.8 & 88.5 & 83.9 \\
\hline Yes prompted & 16.1 & 20.6 & 16.0 & 13.8 & 11.6 & 10.4 & 14.3 \\
\hline No & 0.3 & 0.8 & 0.5 & 1.3 & 1.0 & 0.5 & 0.7 \\
\hline Don't know & 0.5 & 3.3 & 1.6 & 0.3 & 0.5 & 0.5 & 1.0 \\
\hline (N) & (373) & (243) & (382) & $(383)$ & (387) & (393) & $(2,161)$ \\
\hline \multicolumn{8}{|l|}{ Using condom } \\
\hline Yes unprompted & 14.7 & 16.9 & 17.8 & 11.5 & 15.7 & 28.9 & 17.7 \\
\hline Yes prompted & 46.7 & 47.5 & 56.5 & 44.2 & 40.5 & 46.8 & 47.0 \\
\hline No & 16.5 & 11.6 & 15.4 & 24.9 & 25.3 & 10.2 & 17.7 \\
\hline Don't know & 22.1 & 24.0 & 10.2 & 19.4 & 18.6 & 14.1 & 17.6 \\
\hline (N) & $(375)$ & (242) & (382) & $(382)$ & (388) & (391) & $(2,160)$ \\
\hline \multicolumn{8}{|c|}{ Having only one sex partner } \\
\hline Yes unprompted & 19.0 & 10.4 & 38.5 & 25.2 & 14.5 & 45.7 & 26.7 \\
\hline Yes prompted & 58.7 & 65.1 & 53.4 & 68.8 & 75.3 & 48.0 & 61.3 \\
\hline No & 17.7 & 7.5 & 4.7 & 3.9 & 7.3 & 3.8 & 7.4 \\
\hline Don't know & 4.6 & 17.0 & 3.4 & 2.1 & 2.9 & 2.5 & 4.6 \\
\hline (N) & (373) & (241) & (382) & $(381)$ & (385) & (394) & $(2,156)$ \\
\hline \multicolumn{8}{|c|}{ Avoiding sex workers } \\
\hline Yes unprompted & 41.4 & 18.5 & 44.1 & 41.3 & 16.9 & 52.4 & 36.9 \\
\hline Yes prompted & 56.7 & 71.6 & 53.0 & 56.3 & 80.3 & 45.3 & 59.8 \\
\hline No & 1.1 & 2.5 & 1.6 & 1.6 & 2.1 & 1.8 & 1.7 \\
\hline Don't know & 0.8 & 7.4 & 1.3 & 0.8 & 0.8 & 0.5 & 1.6 \\
\hline (N) & (372) & (243) & (379) & $(380)$ & (385) & (395) & $(2,154)$ \\
\hline \multicolumn{8}{|l|}{ Using new needles } \\
\hline Yes unprompted & 52.3 & 18.6 & 42.9 & 50.5 & 33.6 & 45.1 & 41.9 \\
\hline Yes prompted & 46.7 & 68.6 & 54.7 & 48.7 & 63.3 & 52.9 & 55.0 \\
\hline No & 0.5 & 2.1 & 0.8 & & 0.5 & 0.8 & 0.7 \\
\hline Don't know & 0.5 & 10.7 & 1.6 & 0.8 & 2.6 & 1.3 & 2.4 \\
\hline (N) & $(375)$ & $(242)$ & (382) & $(382)$ & (387) & (395) & $(2,163)$ \\
\hline \multicolumn{8}{|c|}{ Abstaining from sex with STI/HIV patients } \\
\hline Yes unprompted & 5.9 & 2.1 & 3.1 & 5.2 & 4.4 & 6.1 & 4.6 \\
\hline Yes prompted & 66.9 & 75.6 & 71.2 & 73.1 & 61.2 & 69.3 & 69.2 \\
\hline No & 15.5 & 2.9 & 13.6 & 16.4 & 26.1 & 13.8 & 15.5 \\
\hline Don't know & 11.7 & 19.4 & 12.0 & 5.2 & 8.3 & 10.7 & 10.7 \\
\hline (N) & (375) & (242) & (382) & (383) & (387) & (391) & $(2,160)$ \\
\hline
\end{tabular}




\section{Awareness and knowledge of other STls (including HIV/AIDS)}

Respondents were asked to list the names of any diseases they had heard of that are transmitted through sexual activity. The results suggest very low levels of knowledge of sexually transmitted diseases other than HIV/AIDS (table 4.9). Without prompting, only two diseases, HIV and hepatitis, were mentioned by the majority of the respondents (72 percent and 44 percent, respectively). The proportion of those mentioning HIV as an STI without prompting was lower than the proportion of those who had heard of the term HIV/ AIDS.

Comparing across cities, the largest proportion of respondents who could not name a single sexually transmitted disease was in Karachi (50 percent), followed by Peshawar and Faisalabad (about 28 percent each). In Rawalpindi, 95 percent of the respondents were able to name at least one sexually transmitted infection. Sozaak (the vernacular name for gonorrhea) was mentioned without prompting as a sexually transmitted infection by 27 percent of the respondents from Quetta, in the other five cities, less than 10 percent of respondents mentioned it by name. Aatshak (the vernacular name for syphilis) was mentioned in all six cities but by less than 10 percent of the respondents in each city. Within the 'other' category, respondents mentioned non-sexually transmitted conditions such as cancer, diabetes, tuberculosis and impotence.

Table 4.9: Percentage distribution of unprompted knowledge of sexually transmitted infections, by city

\begin{tabular}{lrrrrrrr}
\hline STI & Faisalabad & Karachi & Lahore & Peshawar & Quetta & Rawalpindi & Overall \\
\hline None & 28.4 & 50.0 & 15.9 & 28.5 & 13.3 & 5.1 & $\mathbf{2 3 . 6}$ \\
Gonorrhea & 1.3 & 1.5 & 0.0 & 1.3 & 0.8 & 1.5 & $\mathbf{1 . 0}$ \\
Sozaak & 8.0 & 5.0 & 5.3 & 8.5 & 27.3 & 3.3 & $\mathbf{9 . 6}$ \\
Syphilis & 0.8 & 1.3 & 1.0 & 1.8 & 0.8 & 1.3 & $\mathbf{1 . 1}$ \\
Aatshak & 3.5 & 1.0 & 1.8 & 2.3 & 6.5 & 1.3 & $\mathbf{2 . 7}$ \\
Chlamydia & 0.3 & 0.8 & 0.3 & 1.5 & 0.8 & 0.5 & $\mathbf{0 . 7}$ \\
HIV/AIDS & 68.3 & 45.2 & 78.0 & 67.5 & 81.3 & 90.3 & $\mathbf{7 1 . 7}$ \\
Hepatitis & 43.0 & 30.2 & 40.0 & 33.0 & 50.0 & 68.2 & $\mathbf{4 4 . 0}$ \\
Other & 3.3 & 1.5 & 3.3 & 6.0 & 7.3 & 5.9 & $\mathbf{4 . 5}$ \\
(N) & $\mathbf{( 4 0 0 )}$ & $\mathbf{( 4 0 0 )}$ & $\mathbf{( 4 0 0 )}$ & $\mathbf{( 4 0 0 )}$ & $\mathbf{( 4 0 0 )}$ & $\mathbf{( 4 0 0 )}$ & $\mathbf{( 2 , 4 0 0 )}$ \\
\hline
\end{tabular}

After prompting, the diseases that had the highest degree of recognition were HIV/AIDS (89 percent) and hepatitis (87 percent) (table 4.10). About one-third of all of the men recognized the disease sozaak (gonorrhea) (34 percent). Karachi had the 
lowest rates of disease recognition compared to the other cities. When respondents were specifically asked if they had heard the name of the disease sozaak, the highest number of affirmative responses was in Quetta (65 percent) and Peshawar (44 percent), followed by Faisalabad (36 percent), and Lahore (27 percent). The lowest number of responses was in Karachi where only 11 percent of the respondents had heard of the condition. These data support the fact that IEC materials in Pakistan have traditionally been developed to create awareness of HIV/AIDS, and they do not include other sexually transmitted infections.

Table 4.10: Percentage distribution of prompted knowledge of sexually transmitted infections, by city

\begin{tabular}{lrrrrrrr}
\hline STI & Faisalabad & Karachi & Lahore & Peshawar & Quetta & Rawalpindi & Overall \\
\hline Gonorrhea & 5.3 & 2.8 & 6.5 & 3.8 & 3.3 & 7.0 & $\mathbf{4 . 8}$ \\
Sozaak & 36.0 & 10.5 & 26.5 & 44.0 & 64.5 & 20.8 & $\mathbf{3 3 . 7}$ \\
Syphilis & 4.3 & 2.3 & 4.3 & 5.0 & 2.3 & 4.0 & $\mathbf{3 . 7}$ \\
Aatshak & 19.8 & 3.5 & 11.5 & 14.5 & 15.8 & 8.0 & $\mathbf{1 2 . 2}$ \\
Chlamydia & 2.8 & 2.0 & 3.8 & 3.0 & 2.5 & 4.5 & $\mathbf{3 . 1}$ \\
HIV/AIDS & 93.3 & 61.3 & 94.5 & 94.8 & 94.8 & 98.0 & $\mathbf{8 9 . 4}$ \\
Hepatitis & 95.8 & 57.8 & 91.8 & 92.0 & 90.8 & 94.5 & $\mathbf{8 7 . 1}$ \\
(N) & $\mathbf{( 4 0 0 )}$ & $\mathbf{( 4 0 0 )}$ & $\mathbf{( 4 0 0 )}$ & $\mathbf{( 4 0 0 )}$ & $\mathbf{( 4 0 0 )}$ & $\mathbf{( 4 0 0 )}$ & $\mathbf{( 2 , 4 0 0 )}$ \\
\hline
\end{tabular}

Note: The Urdu terminology for diseases was asked separately from the English names (e.g., sozaak and syphilis), and are, therefore, presented separately. Multiple responses were possible.

\section{Provider for treatment of STls}

Respondents were asked where someone would go for treatment for a sexually transmitted infection. Within the category of traditional practice we have grouped faith healers, pirs/sanyasi, and local non-qualified practitioners. Hakeems, practitioners of Unani or Islamic medicine, are a separate category. Except for Faisalabad, respondents in all cities were of the view that men requiring treatment for an STI would most likely visit medical doctors, followed by hakeems. The primary reason given for preferring a medical doctor was that he was adequately qualified to treat STIrelated conditions. This perception of hakeems as being less qualified also emerged from the focus group discussions.

\section{Perception of risk of STIs, including HIV/AIDS}

Respondents were asked if they considered themselves at risk of acquiring a sexually transmitted infection including HIV/AIDS (table 4.11). The highest proportion of respondents who considered themselves at risk was in Faisalabad (29 percent), followed by Peshawar (28 percent), Karachi (26 percent), Quetta (22 percent), and 
Rawalpindi (22 percent). In Lahore, 10 percent of respondents considered themselves to be at risk. Those who considered themselves to be at risk were asked to classify their risk as low, intermediate, or high. Most men classified themselves as low risk (72 percent), as shown in table 4.12 .

Table 4.11: Percentage distribution of men who think they are at risk of acquiring an STI, by city

\begin{tabular}{|c|c|c|c|c|c|c|c|c|c|c|c|c|c|c|}
\hline \multirow[b]{2}{*}{ Response } & \multicolumn{2}{|c|}{ Faisalabad } & \multicolumn{2}{|c|}{ Karachi } & \multicolumn{2}{|c|}{ Lahore } & \multicolumn{2}{|c|}{ Peshawar } & \multicolumn{2}{|c|}{ Quetta } & \multicolumn{2}{|c|}{ Rawalpindi } & \multicolumn{2}{|c|}{ Overall } \\
\hline & $\%$ & $\mathbf{N}$ & $\%$ & $\mathbf{N}$ & $\%$ & $\mathbf{N}$ & $\%$ & $\mathbf{N}$ & $\%$ & $\mathbf{N}$ & $\%$ & $\mathbf{N}$ & $\%$ & $\mathbf{N}$ \\
\hline At risk & 28.8 & 115 & 26.0 & 99 & 10.3 & 41 & 27.6 & 110 & 21.6 & 86 & 21.7 & 86 & 22.6 & 537 \\
\hline
\end{tabular}

Table 4.12: Percentage distribution of men according to their ranking of the risk of acquiring an STI, by city

\begin{tabular}{lrrrrrrr}
\hline Ranking & Faisalabad & Karachi & Lahore & Peshawar & Quetta Rawalpindi & Overall \\
\hline Low & 84.2 & 76.5 & 76.3 & 70.0 & 42.9 & 77.7 & $\mathbf{7 1 . 6}$ \\
Medium & 9.7 & 16.3 & 23.7 & 25.4 & 45.2 & 18.8 & $\mathbf{2 2 . 3}$ \\
High & 6.1 & 7.1 & 0.0 & 4.6 & 11.9 & 3.5 & $\mathbf{6 . 1}$ \\
Total & $\mathbf{1 0 0 . 0}$ & $\mathbf{1 0 0 . 0}$ & $\mathbf{1 0 0 . 0}$ & $\mathbf{1 0 0 . 0}$ & $\mathbf{1 0 0 . 0}$ & $\mathbf{1 0 0 . 0}$ & $\mathbf{1 0 0 . 0}$ \\
(N) & $\mathbf{( 1 1 5 )}$ & $\mathbf{( 9 9 )}$ & $\mathbf{( 4 1 )}$ & $\mathbf{( 1 1 0 )}$ & $\mathbf{( 8 6 )}$ & $\mathbf{( 8 6 )}$ & $\mathbf{( 5 3 7 )}$ \\
\hline
\end{tabular}

Bivariate analysis was conducted to determine whether respondents who considered themselves to be at risk for acquiring STIs were actually involved in any risky sexual behaviors. We took engaging in non-marital sex in the last 12 months as the measure for risky sexual behavior. Fifty-one percent of men who considered themselves to be at risk of STI infection also reported engaging in non-marital sex in the last 12 months (table 4.13).

Table 4.13: Percentage of men considering themselves at risk for STIs and reporting non-marital sex in the last 12 months, by city

\begin{tabular}{lrrrrrrr}
\hline Response & Faisalabad & Karachi & Lahore & Peshawar & Quetta & Rawalpindi & Overall \\
\hline At risk and non- & & & & & & & \\
marital sex & 60.2 & 63.5 & 27.0 & 56.0 & 45.0 & 41.1 & $\mathbf{5 1 . 0}$ \\
(N) & $\mathbf{( 9 8 )}$ & $\mathbf{( 7 4 )}$ & $\mathbf{( 4 5 )}$ & $\mathbf{( 3 4 )}$ & $\mathbf{( 5 8 )}$ & $\mathbf{( 5 6 )}$ & $\mathbf{( 3 6 5 )}$ \\
\hline
\end{tabular}

In Karachi 64 percent of the respondents who reported having non-marital sex in the last 12 months considered themselves to be at risk for acquiring STIs; in Lahore, 27 percent did. The results suggest that while half of the respondents engaging in nonmarital sex considered themselves at risk of acquiring a sexually transmitted infection, they classified this risk as low and hence may continue to engage in risky sexual activities. This suggests that although knowledge is a necessary precondition for 
changing behaviors, it is not the only means of doing so and additional factors may be involved.

\section{Chapter summary}

The data related to men's knowledge and attitudes related to HIV/AIDS and STIs suggest high levels of awareness of HIV/AIDS (90 percent had heard of HIV/AIDS). The main source of knowledge about HIV came from television (84 percent). Those with no education were significantly less likely to have heard of HIV/AIDS (p-value $<0.05)$. This suggests that messages should be provided through media other than television and targeted to those with little or no education.

Despite the high levels of awareness of HIV/AIDS, the study findings suggest poor knowledge about the specific modes of HIV transmission and a persistence of myths and misconceptions about the disease. While there was greater knowledge about sexual route of transmission (85 percent unprompted), knowledge about other modes was low (4 percent unprompted for breastfeeding and 34 percent for needles/sharp objects). The city wise results show variation in levels of knowledge. Karachi had the lowest levels (41 percent could provide no response even after prompting as to transmission modes of HIV/AIDS). Rawalpindi had the highest levels of knowledge, which may reflect the relationship between increased education and socioeconomic status and increased knowledge.

Knowledge about methods of protection against HIV transmission was also low. Eighteen percent of respondents said, without prompting, that condom use was a mode of protection against HIV/AIDS. This low response likely reflects both the perception of condoms as a family planning method and, also, the general lack of knowledge about STI prevention. This lack of knowledge about STI prevention is reflected in the low levels of knowledge of other methods of prevention: 27 percent said unprompted 'having only one sex partner' and 37 percent said unprompted 'avoiding sex workers' were methods of protection against HIV transmission. Nearly a quarter of the respondents had misconceptions regarding the mode of transmission of HIV/ AIDS, mentioning sharing of food, cloths, bedding and toilet as possible modes of transmission.

Knowledge about STIs other than HIV and hepatitis was low. Twenty-four percent of respondents could not, unprompted, name any sexually transmitted infection. 



\section{STI PREVALENCE, SIGNS, SYMPTOMS, AND TREATMENT SEEKING BEHAVIOR}

\section{Prevalence}

Of the 2,396 men who were tested in our study, results for 2,383 specimens were received. Four percent of the individual respondents (106) were diagnosed with an STI on biological testing. These STIs included: syphilis - 1.3 percent (30 cases); HIV - 0.1 percent (3 cases); HSV-2 - 3.4 percent (83 cases). Gonorrhoea and chlamydia testing was done on those respondents who reported having non-marital sex in the last three months. The total number of men tested for gonorrhoea and chlamydia was 256. The prevalence of gonorrhea in these 256 men was less than 1 percent ( 2 cases), while none tested positive for chlamydia.

Figure 5.1 shows the overall and city wise collective STI prevalence, with 95\% CI, in all infected individuals. The total number of STIs diagnosed was 118 in 106 individuals, meaning that 12 individuals in the study were suffering from more than one STIs. (the collective prevalence of microorganisms was 5 percent).

The individual collective prevalence of all infections by all five organisms was 4.4 percent with the highest prevalence in Karachi (8.5 percent), followed by Lahore (5.1 percent), Faisalabad (4.0 percent), Quetta (4.3 percent), Rawalpindi (2.5 percent), and Peshawar (2.0 percent). 
Figure 5.1: STI prevalence $(95 \% \mathrm{Cl})$, by city and overall

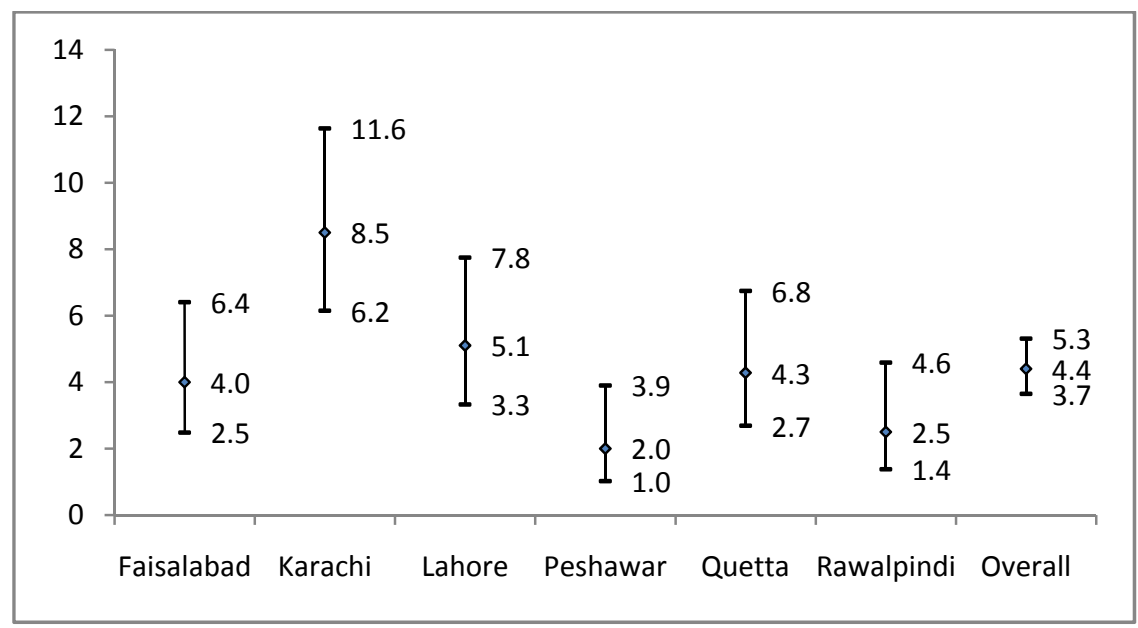

We examined the association between STI presence and demographic characteristics. The results in table 5.1 show significant association between age (defined as less than or greater than 27 years) and STI occurrence (2.8 OR, 95\%CI 1.8-4.4), ${ }^{4}$ with men 27 years or older more likely to be infected. Similarly, there was an association with education; those with ten or fewer years of schooling were more likely to have an STI $(2.7 \mathrm{OR}, 95 \% \mathrm{CI} 1.6$ 4.5). The combination of being married (2.6 OR, 95\%CI 1.7-4.0) and having had non-marital sex in the last three months (1.9 OR, 95\% CI 1.2-3.3.) was also associated with an increased prevalence of an STI. Those respondents reporting four or more non-marital sexual partners were 4.3 (95\% CI 2.3-8.2) Table 5.1: Factors associated with STI prevalence
Unadjusted

\begin{tabular}{lrr} 
Variable & $\begin{array}{r}\text { OR } \\
(95 \% \mathrm{Cl})\end{array}$ & $\begin{array}{r}\text { Adjusted OR } \\
(95 \% \mathrm{Cl})\end{array}$ \\
\hline $\begin{array}{l}\text { Age } \\
>27 \text { years }\end{array}$ & $2.8(1.8-4.4)$ & $2.4(1.3-4.3)$ \\
$<27$ years & 1.0 & 1.0 \\
\hline $\begin{array}{l}\text { Education } \\
\text { Up to 10 years }\end{array}$ & $2.7(1.6-4.5)$ & $2.2(1.3-3.7)$ \\
$>10$ years & 1.0 & 1.0 \\
\hline $\begin{array}{l}\text { Marital status } \\
\text { Currently married }\end{array}$ & $2.6(1.7-4.0)$ & $1.7(0.9-3.1)$ \\
Currently unmarried & 1.0 & 1.0 \\
\hline $\begin{array}{l}\text { Sex in last 3 months } \\
\text { Yes }\end{array}$ & $1.9(1.2-3.3)$ & $0.6(0.2-1.5)$ \\
No & 1.0 & 1.0 \\
\hline $\begin{array}{l}\text { Number of partners } \\
\text { in last 12 months }\end{array}$ & & \\
4 or more & $4.3(2.3-8.2)$ & $11(3.6-33.3)$ \\
3 & $1.9(0.4-8.2)$ & $4.3(0.8-21.9)$ \\
2 & $1.4(0.5-4.1)$ & $3.2(0.9-11.9)$ \\
1 & $1.7(0.9-3.2)$ & $3.3(1.5-7.3)$ \\
None & 1.0 & 1.0 \\
\hline
\end{tabular}
times more likely to have a STI. The reference category in this case was having no partners. After adjustment at multivariate model building, almost all variables lost some strength of their association with STI occurrence, with the exception of 'one' and ' 4 or more' partners which had increased its association as compared to the association

\footnotetext{
${ }^{4} \mathrm{OR}=$ odds ratio
} 
at univariate level. Sex in the last three months was quite significant at univariate level but lost all of its association after adjustment.

\section{STI infection among respondents who report never having sex}

Overall, 9.4 percent $(n=10)$ of all STI positive individuals reported that they had never had penetrative sex. Six of these ten respondents were under the age of 25 . Nine of the ten respondents were positive for HSV-2. Plausible explanations for this could be that respondents were hiding their sexual behavior because of social taboo or shame or it could reflect perinatal transmission as has been shown elsewhere in the literature (Brown, Wald, Morrow et al. 2003; Kasubi, Nilsen, Marsden et al. 2006).

\section{Signs and symptoms}

Respondents were asked about their personal experience of having an STI, including their experience with specific symptoms. These symptoms include pain during urination, purulent discharge from the penis, and ulcer/sores in the genital area. These symptoms can present as one symptom or as a combination of two or more symptoms based on the microorganisms present and the severity of the diseases. Ulcers play an important role in the epidemiology of HIV/AIDS as they can increase transmission of the disease (Gray et al. 2001).

Survey respondents were asked about their experience with three STI symptoms (pain during urination, purulent discharge from the penis, and ulcer/sores in the genital area) over the last six months. They were also asked about their treatment-seeking practices for these symptoms. Among all the respondents, 163 (7 percent) reported experiencing at least one STI symptom during the past six months. Among the cities, Karachi had the highest reporting of any of the three symptoms $(n=40$, or 10 percent of men surveyed in Karachi), while Faisalabad, Rawalpindi, and Peshawar had almost similar proportions of respondents reporting symptoms in the last six months (about 7 percent for each). Lahore had the lowest symptom-reporting rate, with 20 respondents (5 percent) saying they had experienced any of the three STI symptoms in the last six months. 
Of the 163 men reporting STI symptoms, 91 (56 percent) reported pain during urination; the number reporting ulcers/sores in the genital area and purulent discharge was the same (48 men, 29 percent); and 15 percent reported experiencing more than one symptom at the same time during the last six months.

\section{Characteristics of those with STI symptoms}

To further explore the background characteristics of respondents who experienced at least one STI symptom over the last six months, analysis was carried out to define respondents by age, education, marital status, monthly salary earnings, and migration status. These results are shown in table 5.2.

Table 5.2: Characteristics of respondents according to whether they did or did not report STI symptoms in the past six months

\begin{tabular}{lrrr}
\hline & Did report symptom(s) & Did not report symptom(s) & Total \\
\cline { 2 - 4 } Characteristic & Percent & Percent & N \\
\hline Age (in years) & & & $\mathbf{4 6 0}$ \\
$16-20$ & 9.3 & 90.7 & $\mathbf{5 7 3}$ \\
$21-25$ & 8.0 & 92.0 & $\mathbf{4 2 2}$ \\
$26-30$ & 6.6 & 93.4 & $\mathbf{2 8 1}$ \\
$31-35$ & 4.3 & 95.7 & $\mathbf{3 1 3}$ \\
$36-40$ & 6.1 & 93.9 & $\mathbf{3 5 1}$ \\
$41-45$ & 4.3 & 95.7 & $\mathbf{2 , 4 0 0}$ \\
Total & $\mathbf{6 . 8}$ & $\mathbf{9 3 . 2}$ & \\
Educational status & & & $\mathbf{4 3 8}$ \\
No education & 8.2 & 91.8 & $\mathbf{2 5 9}$ \\
Primary & 7.7 & 92.3 & $\mathbf{9 3 6}$ \\
Secondary & 7.5 & 92.5 & $\mathbf{3 3 1}$ \\
High secondary & 4.5 & 95.5 & $\mathbf{4 3 6}$ \\
Bachelor and above & 5.0 & 95.0 & $\mathbf{2 , 4 0 0}$ \\
Total & $\mathbf{6 . 8}$ & $\mathbf{9 3 . 2}$ & \\
\hline Marital status & & & $\mathbf{1 , 2 6 1}$ \\
Married & 5.4 & 94.6 & $\mathbf{1 , 1 3 9}$ \\
Unmarried & 8.3 & 91.7 & $\mathbf{2 , 4 0 0}$ \\
Total & $\mathbf{6 . 8}$ & $\mathbf{9 3 . 2}$ & \\
\hline Income (in Pak Rs.) & & & $\mathbf{1 0 0 . 0}$ \\
Student/unemployed & 7.2 & 92.8 & $\mathbf{1 0 0 . 0}$ \\
Up to 5,000 & 8.4 & 91.6 & $\mathbf{1 0 0 . 0}$ \\
5,001 - 15,000 & 6.2 & 93.8 & $\mathbf{1 0 0 . 0}$ \\
More than 15,000 & 2.6 & 97.4 & $\mathbf{9 3 . 2}$ \\
Total & $\mathbf{6 . 8}$ & & \\
\hline
\end{tabular}




\begin{tabular}{|c|c|c|c|}
\hline & Did report symptom(s) & Did not report symptom(s) & Total \\
\hline Characteristic & $\begin{array}{r}\text { Percent } \\
\end{array}$ & $\begin{array}{r}\text { Percent } \\
\end{array}$ & $\mathbf{N}$ \\
\hline \multicolumn{4}{|c|}{ Migration status } \\
\hline Traveler & 9.0 & 91.0 & 100.0 \\
\hline Migrant & 10.6 & 89.4 & 100.0 \\
\hline Non migrant & 5.9 & 94.1 & 100.0 \\
\hline Total & 6.8 & 93.2 & 100.0 \\
\hline
\end{tabular}

In general, younger respondents reported experiencing STI symptoms the most: 9 percent of those 16-20 years reported symptoms and 8 percent of those 21-25 years of age did so. Those with higher education, bachelor and above, were least likely to report experiencing STI symptoms (5 percent), as opposed to those with no education or up to primary or secondary education (8 percent, respectively). Overall, unmarried men (8 percent) reported STI symptoms more frequently than married men ( 5 percent).

Across all cities, those who earned more than Rs. 15,000 had the lowest proportions of STI symptoms (3 percent). In Faisalabad, Peshawar, and Quetta, STI symptoms were reported more frequently by those earning Rs. 5,000 per month, while in Karachi and Lahore symptoms were more frequent among those with monthly salary between Rs. 5,001 - 15,000. Overall, students and unemployed men were in the highest proportion experiencing STI symptoms in Rawalpindi. (For tables on characteristics of respondents, see appendix 1.)

Migrants (defined as those living away from their marital or natal home for longer than 4 months) reported experiencing STI symptoms more than non-migrants (11 percent versus 6 percent). Nine percent of travelers (those men who spend at least one night out of the home for business) also reported experiencing STI symptoms.

\section{Symptoms and sexual behavior}

Figure 5.2 provides information on the presence of STI symptoms in the last six months among respondents who had non-marital sexual intercourse in the 3 months and 12 months preceding the interview. 
Figure 5.2: STI symptoms among those who had non-marital sex within a specified time prior to the interview

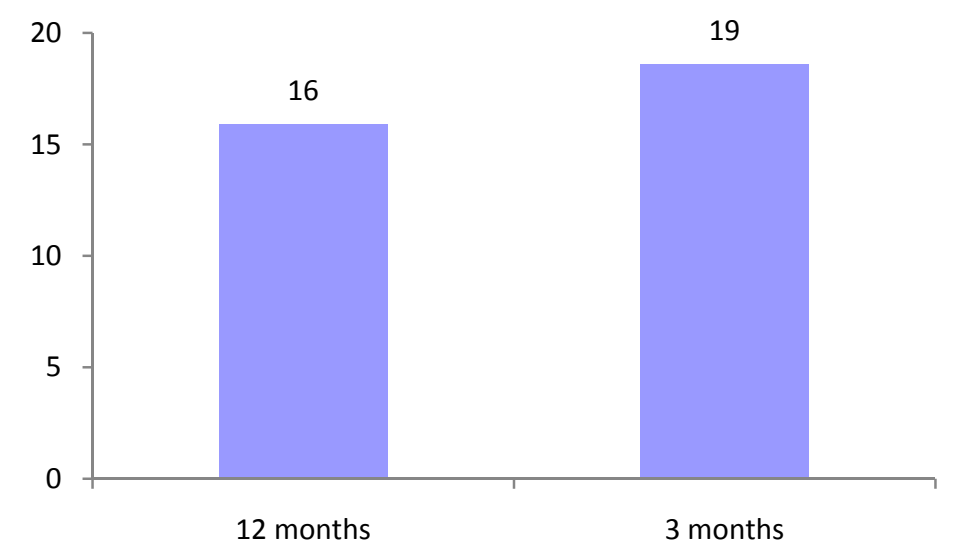

As figure 5.2 shows, self-reported prevalence of STI symptoms increased as the time of sexual intercourse was closer to the time of conducting the questionnaire. STI symptoms were reported in 19 percent of those who had non-marital sexual intercourse in the last three months, as compared to 16 percent among those who reported non-marital sexual intercourse in the last 12 months.

\section{Symptoms and condom use}

To look for the frequency of condom use among those respondents who reported STI symptoms, cross tabulation of STI symptoms by condom use at last nonmarital sexual intercourse ${ }^{5}$ was carried out.

Table 5.3: Use of condom at last nonmarital sexual intercourse among those reporting any STI symptoms

\begin{tabular}{lrr}
\hline & \multicolumn{2}{c}{ Reported STI symptom(s) } \\
\cline { 2 - 3 } Condom use & $\%$ & N \\
\hline Used condom & 16.6 & 27 \\
Did not use condom & 83.4 & 136 \\
Total & $\mathbf{1 0 0 . 0}$ & $\mathbf{1 6 3}$ \\
\hline
\end{tabular}

Table 5.3 depicts the frequency of condom use at last non-marital sexual intercourse among those respondents who reported experiencing STI symptoms. Out of a total of 163 respondents who had reported STI symptoms, 27 (17 percent) had used condoms at last sexual intercourse. A substantially higher proportion of men who reported experiencing STI symptoms had not used condoms at last sexual intercourse (83 percent).

\footnotetext{
${ }^{5}$ We took last intercourse because the question regarding STI symptoms was also asked for the period of the last six months.
} 


\section{Symptoms, diagnosis and care-seeking behavior}

This section describes the respondents' personal experiences with the three STI symptoms (pain during urination, purulent discharge, and genital ulcer/sores) and their care-seeking behaviors.

Table 5.4 describes whether or not a particular STI symptom was diagnosed; and if so, how it was diagnosed. In the majority of all three symptoms, the problem was not diagnosed. The most common diagnosis was gonorrhea (sozaak) based on the symptoms of purulent discharge and pain during urination. In most cases, the problem was diagnosed based on personal history/signs and symptoms. This is especially true in the case of ulcers/sores, where 88 percent of the cases were diagnosed on the basis of symptoms. Laboratory tests were used for diagnosis in 15 percent of the purulent discharge cases and 37 percent in the case of pain during urination. A discussion of laboratory results appears at the end of this section.

Table 5.4: Diagnostic outcomes based on STI symptom

\begin{tabular}{|c|c|c|c|c|c|c|}
\hline \multicolumn{7}{|c|}{ STI Symptom } \\
\hline & \multicolumn{2}{|c|}{ Ulcer/Sores } & \multicolumn{2}{|c|}{ Purulent discharge } & \multicolumn{2}{|c|}{ Pain during urination } \\
\hline & $\%$ & $\mathrm{~N}$ & $\%$ & $\mathrm{~N}$ & $\%$ & $\mathrm{~N}$ \\
\hline \multicolumn{7}{|l|}{ No diagnosis } \\
\hline Problem not diagnosed & 22.2 & 8 & 36.4 & 12 & 25.0 & 14 \\
\hline Was not told anything & 8.3 & 3 & 9.1 & 3 & 1.8 & 1 \\
\hline Self-medication & 2.8 & 1 & 3.0 & 1 & 3.6 & 2 \\
\hline \multicolumn{7}{|l|}{ Problem diagnosed as: } \\
\hline Gonorrhea (Sozaak) & 2.8 & 1 & 18.2 & 6 & 17.9 & 10 \\
\hline Syphilis (Aatshak) & 5.6 & 2 & 6.1 & 2 & 3.6 & 2 \\
\hline Chlamydia & 2.8 & 1 & 3.0 & 1 & 1.8 & 1 \\
\hline Hepatitis & 2.8 & 1 & 6.1 & 2 & 1.8 & 1 \\
\hline Allergy & 8.3 & 3 & 3.0 & 1 & 1.8 & 1 \\
\hline Stone & 5.6 & 2 & 3.0 & 1 & 3.6 & 2 \\
\hline Burning in bladder & 5.6 & 2 & 3.0 & 1 & 10.7 & 6 \\
\hline Jaryan" & 5.6 & 2 & 3.0 & 1 & 8.9 & 1 \\
\hline Wound in urethra & 5.6 & 2 & 3.0 & 1 & 7.1 & 4 \\
\hline Number of respondents & 100 & 36 & 100 & 33 & 100 & 56 \\
\hline \multicolumn{7}{|c|}{ How problem was diagnosed } \\
\hline History/sign symptoms & 75.0 & 27 & 54.5 & 18 & 37.5 & 21 \\
\hline Lab test & 5.6 & 2 & 12.1 & 4 & 28.6 & 16 \\
\hline Self estimated & 0.0 & 0 & 6.1 & 2 & 3.6 & 2 \\
\hline Service provider & 2.8 & 1 & 0.0 & 0 & 0.0 & 0 \\
\hline No information & 16.7 & 6 & 27.3 & 9 & 30.4 & 17 \\
\hline Number of respondents & 100 & 36 & 100 & 33 & 100 & 56 \\
\hline
\end{tabular}


*Jaryan is the emission of seminal fluid.

Multiple response questions so total may go beyond $100 \%$.

Treatment-seeking behavior, below, is presented according to the STI symptoms of pain during urination, purulent discharge from the penis and ulcer/sores in the genital area.

Ulcers/sores in genital area. There were a total of 48 respondents ( 2 percent) in all cities who reported experiencing ulcers/sores in their genital area in the last six months. Peshawar and Faisalabad each had 3 percent of the respondents who reported this symptom - the highest among all cities - while Quetta had the lowest percentage (less than 1 percent).

In Lahore, all respondents with ulcers/sores sought treatment ( $\mathrm{n}=5,100$ percent), while the majority of respondents in Faisalabad ( $n=11,85$ percent), Karachi $(n=8,73$ percent), and Peshawar ( $n=9,69$ percent) also reported seeking treatment. As with other STI symptoms, most respondents sought treatment from a doctor or consulted a traditional practitioner. Those who did not seek treatment said they were shy or were not aware of treatment.

Fewer respondents reporting genital ulcer sores than the other two symptoms said that lab tests had been used to make a diagnosis (5.6 percent). However, when compared to the other two symptoms, more respondents with genital ulcers said their partners were aware of the disease (39 percent). This may be due to the obvious physical manifestation of this symptom. Still, more than half of the respondents (52 percent) did nothing to prevent their partner from getting the disease, while almost one-fifth (19 percent) said they avoided sex. Only one respondent said he used a condom to prevent his partner from getting the disease.

Table 5.5: Treatment Seeking Behavior for STI Management

\begin{tabular}{lrrrrrr}
\hline & \multicolumn{2}{c}{ Ulcer/Sores } & \multicolumn{2}{c}{ Purulent discharge } & \multicolumn{2}{c}{ Pain during urination } \\
\cline { 2 - 7 } & \multicolumn{1}{c}{$\%$} & $\mathrm{~N}$ & $\%$ & $\mathrm{~N}$ & $\%$ & $\mathrm{~N}$ \\
\hline Did seek treatment & & & & & & \\
Yes & 75.0 & 36 & 68.8 & 33 & 61.5 & 56 \\
No & 25.0 & 12 & 31.3 & 15 & 38.5 & 35 \\
Number of respondents & 100.0 & $\mathbf{4 8}$ & $\mathbf{1 0 0 . 0}$ & $\mathbf{4 8}$ & $\mathbf{1 0 0 . 0}$ & $\mathbf{9 1}$ \\
\hline Where sought treatment $^{*}$ & & & & & & \\
Doctor & 63.9 & 23 & 56.3 & 18 & 62.3 & 33 \\
Dispenser/Paramedic & 5.6 & 2 & 3.1 & 1 & 1.9 & 1 \\
Traditional Practitioner & 30.6 & 11 & 65.6 & 21 & 41.5 & 22
\end{tabular}




\begin{tabular}{|c|c|c|c|c|c|c|}
\hline & \multicolumn{2}{|c|}{ Ulcer/Sores } & \multicolumn{2}{|c|}{ Purulent discharge } & \multicolumn{2}{|c|}{ Pain during urination } \\
\hline & $\%$ & $\mathrm{~N}$ & $\%$ & $\mathrm{~N}$ & $\%$ & $\mathrm{~N}$ \\
\hline Self Medication & 11.1 & 4 & 3.1 & 1 & 15.1 & 8 \\
\hline No information & 0.0 & 0 & 3.1 & 1 & 5.3 & 3 \\
\hline Number of respondents & & 36 & & 33 & & 56 \\
\hline \multicolumn{7}{|l|}{ Why did not seek treatment ${ }^{\star}$} \\
\hline Not aware & 25.0 & 3 & 6.7 & 1 & 20.0 & 7 \\
\hline Facility not available & 16.7 & 2 & 6.7 & 1 & 5.7 & 2 \\
\hline Financial barriers & 8.3 & 1 & 13.3 & 2 & 20.0 & 7 \\
\hline Shyness & 25.0 & 3 & 26.7 & 4 & 17.1 & 6 \\
\hline Didn't think it necessary/Didn't have time & 16.7 & 2 & 26.7 & 4 & 34.3 & 12 \\
\hline Side effects of medicine & 33.3 & 4 & 20.0 & 3 & 20.0 & 7 \\
\hline No information & 8.3 & 1 & 6.7 & 1 & 5.7 & 2 \\
\hline Number of respondents & & 12 & & 15 & & 35 \\
\hline \multicolumn{7}{|c|}{ Preventive measures used to prevent partner from getting the disease } \\
\hline Nothing & 52.1 & 25 & 60.4 & 29 & 53.8 & 49 \\
\hline Avoid intercourse & 18.8 & 9 & 8.3 & 4 & 5.5 & 5 \\
\hline Used condom & 2.1 & 1 & 6.3 & 3 & 5.5 & 5 \\
\hline Never had sex & 8.3 & 4 & 6.3 & 3 & 7.7 & 7 \\
\hline Partner's treatment & 0.0 & 0 & 2.1 & 1 & 1.1 & 1 \\
\hline No information & 18.8 & 9 & 16.7 & 8 & 30.8 & 28 \\
\hline Number of respondents & 100.0 & 48 & 100.0 & 48 & 100.0 & 91 \\
\hline \multicolumn{7}{|l|}{ Partner was aware about the disease } \\
\hline Yes & 38.9 & 14 & 15.2 & 5 & 30.4 & 17 \\
\hline No & 36.1 & 13 & 60.6 & 20 & 39.3 & 22 \\
\hline No information & 25.0 & 9 & 24.2 & 8 & 30.4 & 17 \\
\hline Number of respondents & 100.0 & 36 & 100.0 & 33 & 100.0 & 56 \\
\hline
\end{tabular}

*Multiple responses were allowed.

Purulent discharge. Out of the study respondents, 48 (2 percent) reported experiencing the symptom of purulent discharge in the last six months. Karachi had the relatively highest proportion of respondents ( 5 percent) reporting this symptom, followed by Rawalpindi ( 2 percent), Quetta ( 2 percent), Lahore ( 2 percent), Faisalabad (1 percent) and Peshawar (1 percent).

Among those who experienced purulent discharge, 69 percent sought treatment for the problem (table 5.5). In Quetta, Lahore, and Rawalpindi, higher proportions sought treatment for this problem (86 percent, 83 percent, and 63 percent, respectively). These proportions were higher than those seeking treatment for pain during urination, suggesting that perhaps respondents considered this symptom as more serious. 
Respondents mostly sought care from doctors and/or traditional practitioners. In Rawalpindi and Lahore, the preferred health care providers were traditional practitioners.

All respondents in Lahore reported financial barriers as the main reason for not seeking care. In Peshawar, all respondents who did not seek care cited shyness as the reason. In Quetta, unavailability of facility and shyness were the main factors. In Rawalpindi, shyness was cited by half of the respondents, and in Karachi a similar proportion of respondents (14 percent) cited unavailability of facility, financial barriers, and shyness as restricting factors. Tables presenting city-wise analysis are found in appendix 1.

In Peshawar, none of the respondents who had experienced purulent discharge reported that any diagnosis had been made. In Karachi, 47 percent of the respondents reported that no diagnosis was made. In both Faisalabad and Quetta, half of the respondents were diagnosed with gonorrhea. In Rawalpindi, gonorrhea was diagnosed in 20 percent of the respondents. The majority of diagnoses (88 percent) were made without a lab test (table 5.4). Most men suffering from purulent discharge did not tell their partner (61 percent), and 60 percent did nothing to prevent transmission to their partner (table 5.5).

Pain during urination. A total of 91 respondents (3.8 percent) reported experiencing pain during urination in the last six months. Not all of the respondents with pain during urination sought treatment for this symptom, and there was a range across cities, from 77 percent in Quetta to 44 percent in Rawalpindi. In most cities, a medical doctor was the preferred health care provider for managing pain during urination, except in Lahore where respondents preferred traditional practitioners. In Quetta, 83 percent of the respondents preferred a medical doctor; in the other cities, more than two-thirds (67 percent) reported visiting the doctor. Except for Lahore, traditional practitioners were not visited by a significant proportion of respondents. Dispenser/paramedics were the least consulted care providers for pain during urination (table 5.5). Reasons for not seeking treatment for pain during urination included financial barriers and shyness both with similar proportion (11 percent).

In Karachi and Lahore, half of the respondents reported that their condition was not diagnosed. In 18 percent, the disease was diagnosed as gonorrhea (sozaak), and in a few cases the diagnosis of hepatitis was made. Across all cities, 39 percent of partners 
were unaware of the diagnosis, and 54 percent of the respondents did nothing to prevent transmission to their partner (table 5.5).

\section{Symptoms of STls and biological testing}

Those who reported STI symptoms were analyzed based on the results of the biological laboratory testing done on the blood and urine specimens received from the respondents. This analysis, by type of symptoms, shows that among those who complained of purulent discharge $(n=48)$, four were positive for any micro-organism $(8$ percent); this percentage was the highest of the three STI symptoms. Four percent of those who reported ulcers or sores in the genital area were found positive for an STI, and 3 percent of those who reported pain during urination tested positive for an STI. This suggests that purulent discharge is the most specific of the three STI symptoms. Ninety-one of the total 163 men who reported symptoms complained of pain during urination, making this the least specific symptom.

Table 5.6: Cross-tabulation of specific STI symptoms with confirmed STI on biological testing

\begin{tabular}{lrr}
\hline Symptom & $\begin{array}{r}\text { Number of confirmed STI } \\
\text { cases by lab results }\end{array}$ & \begin{tabular}{c} 
Number reporting symptoms \\
\hline Ulcers/sores in the genital area
\end{tabular} \\
Purulent discharge from the penis & 2 & 48 \\
Pain during urination & 3 & 48 \\
(N) & $\mathbf{9}$ & 91 \\
\hline
\end{tabular}

${ }^{a} \mathrm{~N}=163$. This is the number of respondents, not the number of symptoms; men could report more than one symptom.

One hundred sixty-three respondents reported having at least one of three STI symptoms (either pain during urination, purulent discharge, or genital ulcer). As stated earlier, 106 (4 percent) of all respondents were found positive for at least one STI on biological testing (table 5.7). We found that among these, only nine (9 percent) were found positive on biological testing. We calculated the sensitivity of using selfreported STI symptoms as a marker for STI infection and found it to be 9 percent (nine out of 106 with laboratory confirmed STI infections). The specificity of self-reported STI symptoms was 93 percent. There are several explanations for this finding. First, we inquired about the occurrence of symptoms in the last six months and 70 percent of the respondents reported having received treatment, which would influence the biological results. 
Table 5.7: Distribution of respondents who did or did not report any STI symptoms according to laboratory findings of the presence of an STI

\begin{tabular}{lrrrrrr}
\hline & \multicolumn{3}{c}{$\begin{array}{l}\text { Men who reported any } \\
\text { symptom(s) }\end{array}$} & $\begin{array}{r}\text { Men who reported no } \\
\text { symptom(s) }\end{array}$ & Total \\
\cline { 2 - 7 } Laboratory diagnosis & $\%$ & $\mathbf{N}$ & $\%$ & $\mathbf{N}$ & $\%$ & $\mathbf{N}$ \\
\cline { 2 - 8 } STI present & 8.5 & 9 & 91.5 & 97 & 4.4 & 106 \\
STI not present & 6.7 & 154 & 93.3 & 2,140 & 95.6 & 2,294 \\
Total & $\mathbf{6 . 8}$ & $\mathbf{1 6 3}$ & $\mathbf{9 3 . 2}$ & $\mathbf{2 , 2 3 7}$ & $\mathbf{1 0 0 . 0}$ & $\mathbf{2 , 4 0 0}$ \\
\hline
\end{tabular}

Despite the explanations for the low sensitivity, these results have important implications for the use of self-reported STI symptoms in the diagnosis and treatment of STIs. Ninety-two percent of men who had STIs did not report experiencing any symptoms. These findings suggest that without laboratory testing, these men would have gone undiagnosed and perhaps never been aware they had an STI. This clearly has important implications for STI transmission as those men who are asymptomatic and unaware of their disease status will not take measures to prevent transmitting disease to their partner. This also suggests limitations of the STI syndromic management approach, which is entirely based on self-reporting of symptoms.

\section{Chapter summary}

The STI prevalence results presented in this section are within the predicted range and are similar to other studies of STI prevalence in South Asia (Hawkes et al. 1999; Faisel and Cleland 2006). One hundred and six men in the study population had at least one STI on biological testing (4 percent prevalence rate). Fourteen of these men tested positive for more than one STI, which gives a 5 percent prevalence rate based on all infections. The most common STI was HSV-2 (3 percent prevalence) with syphilis (1 percent) and HIV (0.1 percent) the next most common. It is possible that these results are affected by the marker used in the study (infection versus exposure) and the fact that 70 percent of men who experienced STI symptoms in the past six months had sought some form of treatment for them. The HIV prevalence rate of 0.01 percent reported in this study is similar to what has been reported in the general population (World Bank 2006).

Based on unadjusted odds ratios, the factors associated with testing positive for an STI include being over the age of 27, which increases the odds of having an STI by 2.8 times; having ten years or less of education (2.7 unadjusted OR); having non-marital sex in the last three months (1.9 unadjusted OR), having more than four partners in the last 12 months (4.3 unadjusted OR) and being married (2.6 unadjusted OR). 
The data show that 163 men (7 percent) experienced at least one STI symptom in the past six months. The most common symptom was pain during urination (56 percent), followed by genital ulcers/sores ( 29 percent) and purulent discharge ( 29 percent). Fifteen percent had more than one symptom. Despite these symptoms, a problem was not diagnosed in roughly one-third of the men with symptoms. Of these men, most were diagnosed based on symptoms and medical history rather than laboratory testing. Treatment-seeking behavior was different depending on the STI symptom. Seventy-five percent of those men with genital ulcers/sores sought treatment; 70 percent with purulent discharge sought treatment; and 60 percent complaining of pain during urination sought treatment. Those who did not seek treatment said that shyness and lack of awareness were important factors in preventing them from seeking treatment.

The majority of men who experienced STI symptoms did nothing to prevent their partner from getting the disease. In about one-half of those men reporting STI symptoms, partners remained unaware that he had an STI symptom. Ninety percent of men with symptoms did not use a condom at their last sexual intercourse.

The analysis suggests that STI symptoms have low diagnostic sensitivity; 9 percent of those experiencing symptoms were confirmed as having an STI based on laboratory result. Out of the three STI symptoms, purulent discharge, was the most sensitive (8 percent), followed by genital ulcers/sores (4 percent), and pain during urination (3 percent). 



\section{SEXUAL AND NONSEXUAL BEHAVIORS RELATED TO STI PREVALENCE AND TRANSMISSION}

\section{Sexual behavior}

In this section we look at three areas to better understand the general context of sexual behavior: 1) sexual debut; 2) non-marital sexual behaviors, looking at behavior in the respondent's lifetime, in the last 12 months and the last 3 months; and 3) frequency of condom use. We then present descriptive statistics with 95 percent confidence intervals and multivariate analyses to identify the specific characteristics of those engaging in particular non-marital sexual behaviors in the last 12 months. This will allow us to identify the specific characteristics of the bridging population in the next chapter. Also included in this chapter is information from the qualitative research. This is included here because it provides further insight into the attitudes of men regarding their nonmarital sexual behaviors.

\section{Sexual debut}

This section describes the sexual debut ${ }^{6}$ of the respondents and analyzes variations in their sexual partners and age groups. Figure 6.1 shows the results for sexual debut. Among the total sample of 2,400 men, 31 percent reported never experiencing marital or non-marital sexual intercourse (i.e., were virgins), while 42 percent reported having had their first sexual encounter with their wives. Twenty-seven percent $(n=653)$ reported having premarital sex.

\footnotetext{
${ }^{6}$ The term "sexual debut" refers to the first experience with any penetrative sex.
} 
Figure 6.1: Sexual debut, by type of partner

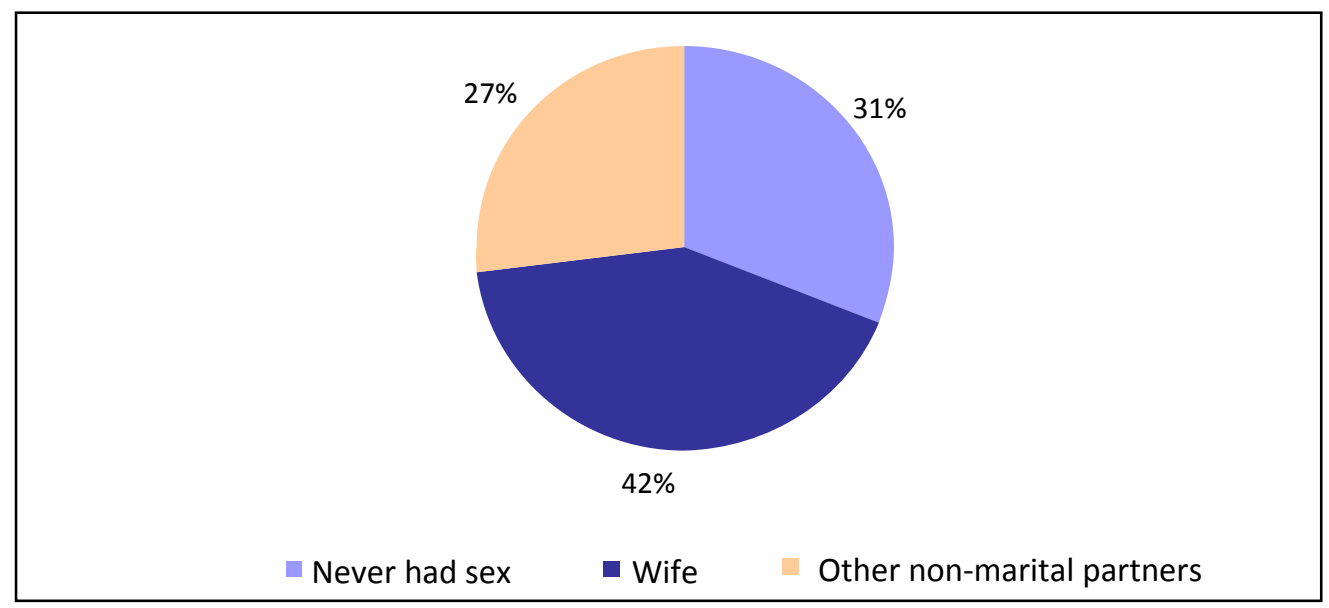

Figure 6.2 shows non-marital sexual debut by type of partner. Of the 653 respondents (27 percent) who reported having a premarital sexual encounter as their first sexual experience, 53 percent did so with a female other than a sex worker (FOSW),28 percent did so with a female sex worker, 3 percent with a male sex worker, and 17 percent with a male other than sex worker and/or hijra (only 3 respondents, 0.5 percent admitted having had sex for the first time with a hijra).

Figure 6.2: Non-marital sexual debut, by type of partner (percent)

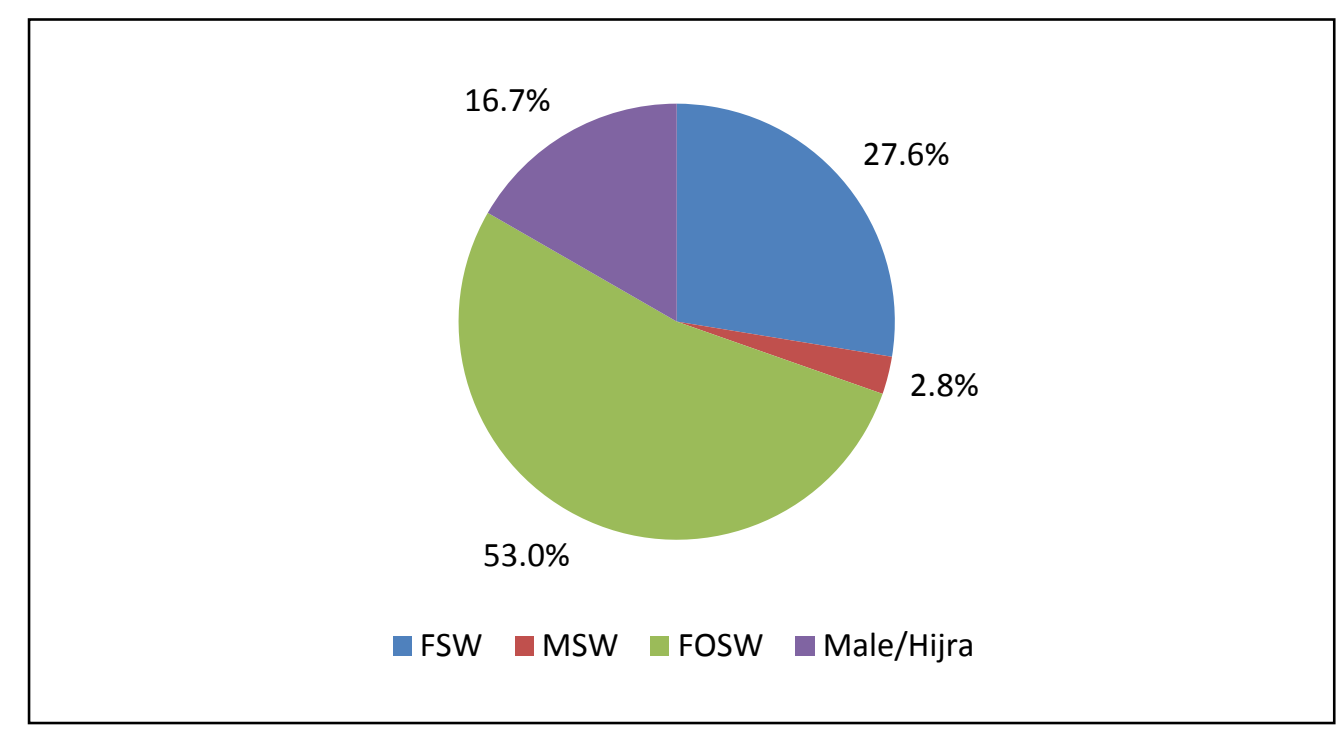


Age distribution at first intercourse. Figure 6.3 shows the age distribution at first intercourse. The mean and median age at the time of marital sexual debut was 22 years. The mean and median ages at the time of sexual debut for pre-marital sex were 19 and 18 years, respectively.

Overall, the majority of men (44 percent) had their first intercourse between the ages of 16-20 years. Seventy-four percent of men had their first sexual experience by the age of 26. At older age bands the percentages decrease. Seven percent reported first sexual experience at the ages of 31-35 years; less than 1 percent had their first intercourse between the ages of 36-45 years.

Figure 6.3: Age distribution at first intercourse (percent)

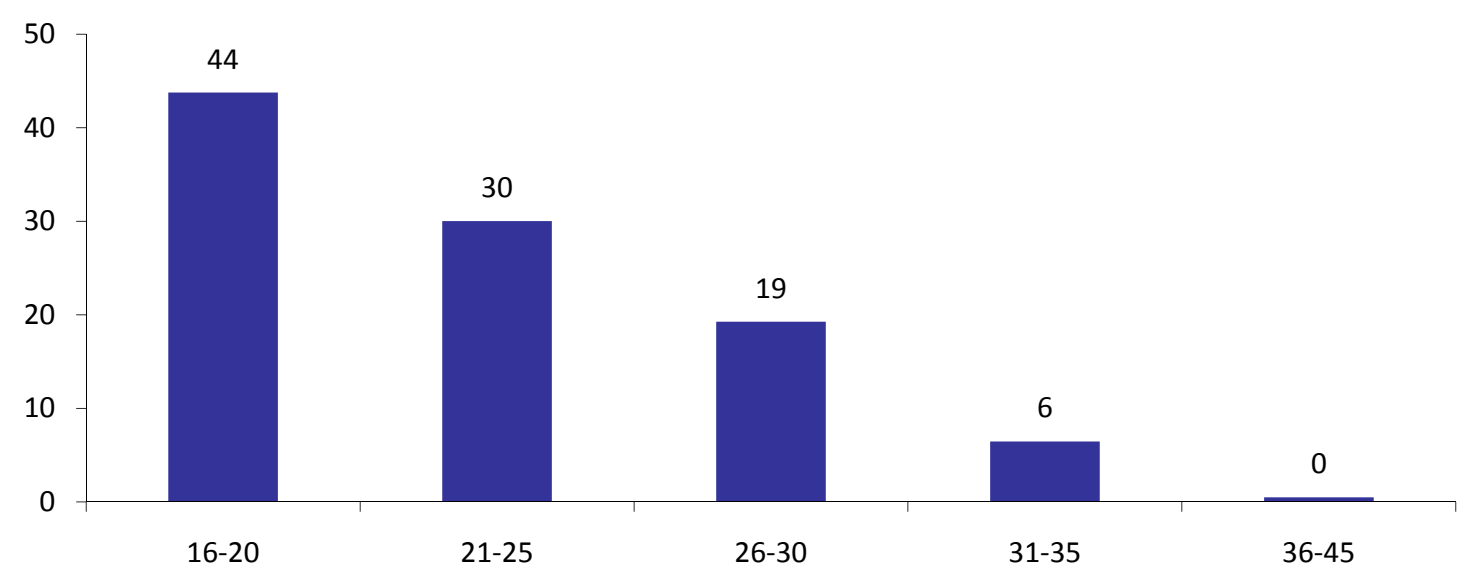

Figure 6.4 shows the variation in type of sexual debut partners (other than wife) among different age groups. Sex with males/hijras is more likely to occur at younger ages (16-20 years) and declines with age. At younger ages men often begin sexual experimentation, and their choice of sexual partner at this age is often based on availability and affordability. This may explain why male sexual partners are reported comparatively higher in the lower age groups than in the older ones. 
Figure 6.4: Type of partner, by age at first intercourse

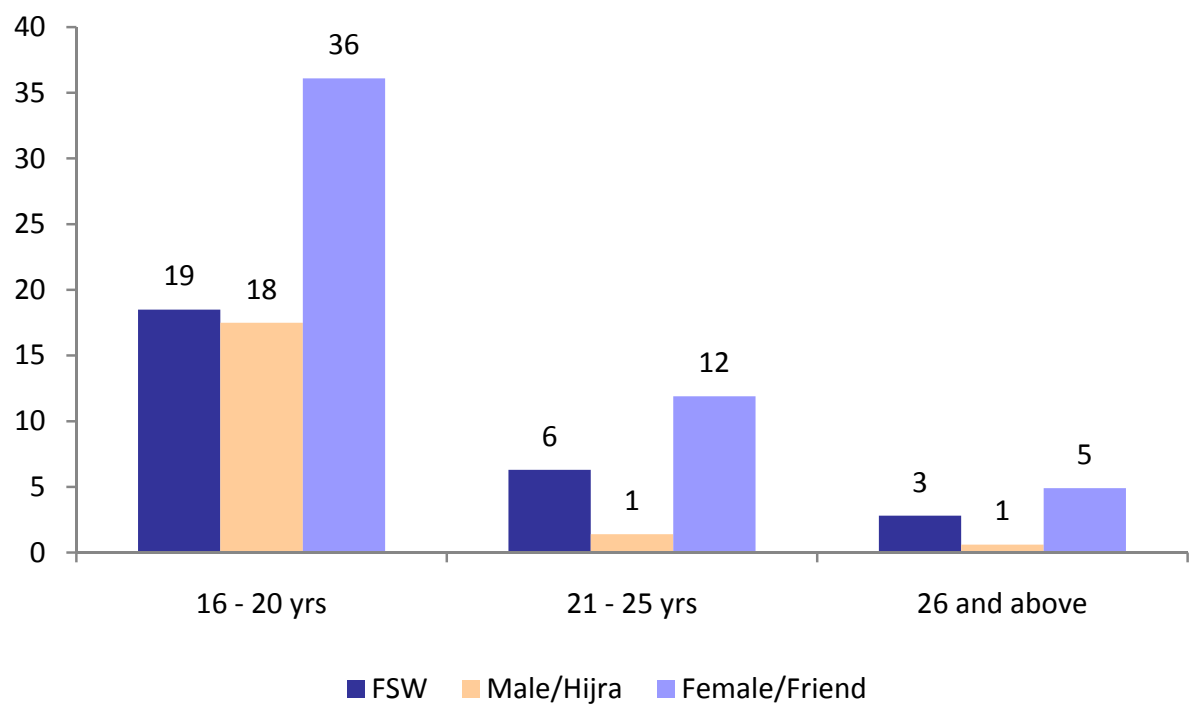

\section{Non-marital sexual behaviors}

In order to determine the extent to which the study population engages in premarital or extramarital sexual activity, all respondents were asked to recall their non-marital sexual experiences over their lifetime, within the last twelve and three months. The results, which include all men in the study, are shown in figure 6.5. Lifetime number of sexual partners provides insight into the overall exposure of a person to sexually transmitted infections; however, this information can be influenced by issues of recall due to age and exposure period.

Figure 6.5: Lifetime non-marital sexual behavior of all respondents

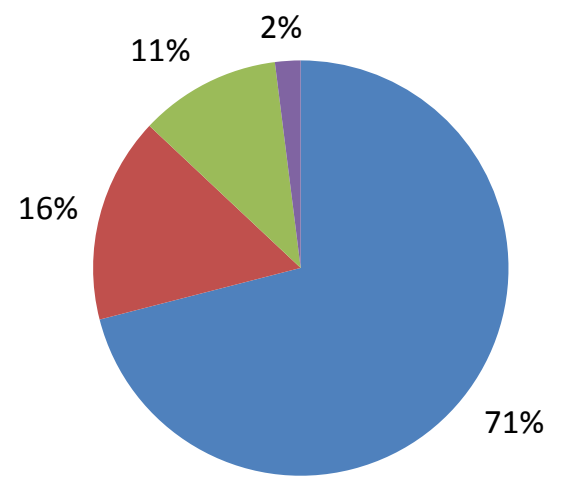

Never had non-marital sex $\quad$ Premarital sex(unmarried)

$\square$ Pre \& extra marital sex (married) $\square$ Extramarital sex only 
Nearly one third (29 percent) reported having had non-marital sex in their lifetime. Of these men, 16 percent reported premarital sex, while 11 percent reported engaging in both pre- and extramarital sex. Only two percent reported exclusive extramarital sex. In total, 13 percent of men reported engaging in extramarital sexual activity. These results are supported by other studies in the South Asian context that found relatively high rates of both extramarital and premarital sexual activity. Collumbien's (2000) study among urban and rural men in India found that 25 percent had sex before marriage, and did so with a variety of partners (e.g., sex workers, friends, relatives, and future spouses). Faisel and Cleland (2006) found that 55 percent of the unmarried men in their study of migrant men in Lahore reported being sexually experienced.

Figure 6.6 shows non-marital sexual behavior over different time periods (lifetime, 12 months, and 3 months), with 95\% confidence intervals. Eleven percent of respondents reported non-marital sex in the last three months, fifteen percent in the last 12 months and 29 percent reported non-marital sex ever in their lifetime.

Figure 6.6: Respondent's non-marital sexual behavior, over three time periods (with 95\% Cl)

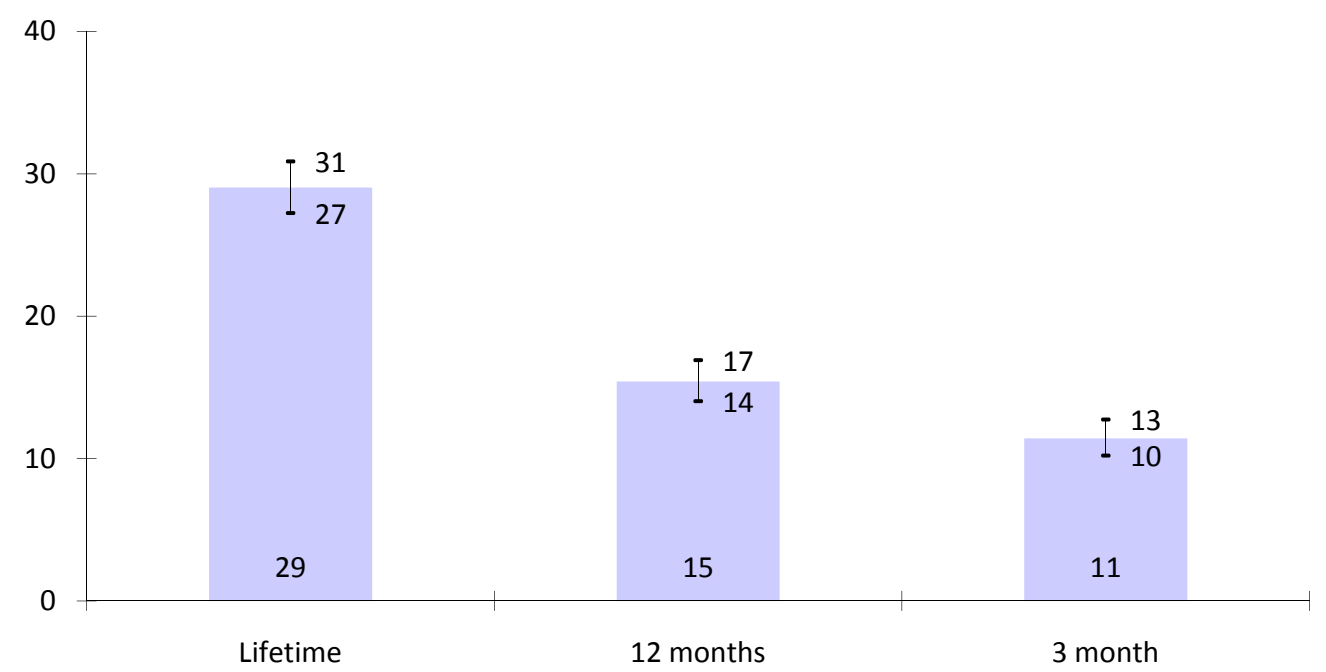

Table 6.1 presents the lifetime distribution of sexual partners by type. It shows that among 697 respondents who had ever had non-marital sex in their lifetimes, 65 percent reported having sex with a female partner who was not a sex worker and that no financial transaction took place in exchange for sex. These partners in most instances were labeled by respondents as 'friends.' These sexual partners have also been reported in the earlier study by Faisel and Cleland (2006). The concept of unpaid sex with these friends needs further elaboration. Our qualitative research shows that 
although these relationships may not be purely financially mediated there is some degree of transaction 'in kind' -- which could range from providing gifts or doing favors. The next most frequent category was female sex workers (41 percent overall). This was reported the most in Quetta (52 percent) and Lahore (45 percent). The lowest proportion of respondents reporting sex with a female sex worker was in Rawalpindi (35 percent). The third most frequent non-marital sexual liaison was reported with males who were not sex workers. The highest proportion reported in this category was in Rawalpindi (32 percent), followed by Peshawar (28 percent); the lowest was in Lahore (10 percent). The highest proportion reporting sex with a male sex worker overall was 8 percent; the highest was reported in Karachi (16 percent) and the lowest was in Lahore where only two respondents reported such a liaison.

Sex with eunuch sex worker (hijra) was about 6 percent in all six cities. The highest proportion reporting sex with hijras was in Faisalabad (7 percent), followed by Karachi (6 percent). The lowest numbers were in Lahore and Rawalpindi, where only three respondents in each city reported such a liaison. It is possible that there is underreporting of sex with hijras due to the stigma against sex with these individuals (Faisel and Cleland 2006).

Table 6.1: Type of sexual partners of those who had non-marital sex in lifetime, by city

\begin{tabular}{lrrrrrrr}
\hline Partner & Faisalabad & Karachi & Lahore & Peshawar & Quetta & Rawalpindi & Overall \\
\hline FOSW & 71.5 & 58.3 & 71.7 & 62.1 & 53.5 & 70.4 & 64.8 \\
FSW & 34.8 & 42.4 & 45.3 & 37.9 & 51.5 & 34.7 & 40.7 \\
MSW & 5.1 & 15.8 & 1.9 & 6.3 & 10.9 & 8.2 & 8.2 \\
NMSW & 25.3 & 20.9 & 10.4 & 28.4 & 23.8 & 31.6 & 23.2 \\
Hijra & 7.0 & 6.5 & 2.8 & 8.4 & 5.9 & 3.1 & 5.7 \\
(N) & $\mathbf{( 1 5 8 )}$ & $\mathbf{( 1 3 9 )}$ & $\mathbf{( 1 0 6 )}$ & $\mathbf{( 9 5 )}$ & $\mathbf{( 1 0 1 )}$ & $\mathbf{( 9 8 )}$ & $\mathbf{( 6 9 7 )}$ \\
\hline
\end{tabular}

Looking at the distribution of high-risk partners within the married and unmarried categories, the results suggest that unmarried men had higher rates of sexual activity with male sex workers, males other than sex workers, and hijras as compared to married men (figure 6.7). Most unmarried men were in the younger age category, when, as suggested earlier, sexual experimentation is quite common irrespective of the type of partner. 
Figure 6.7: Type of high-risk sexual partner, by marital status (percent)

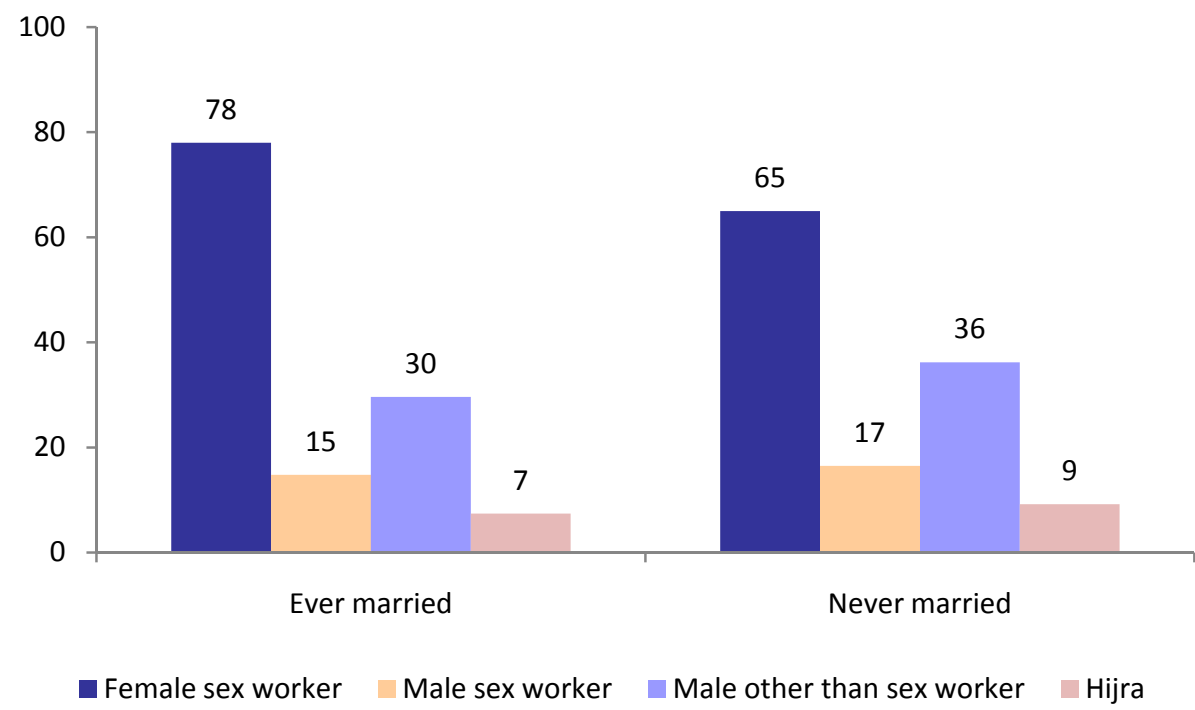

Table 6.2 shows that overall 44 percent of those who reported non-marital sex said they had three or more non-marital partners in their lives, while 39 percent reported having one partner only. Having multiple sexual partners enhances the risk of acquiring STIs and should therefore be a focus of future Information, Education and Communication (IEC) campaigns.

Table 6.2: Number of lifetime non-marital sexual partners, by city

\begin{tabular}{|c|c|c|c|c|c|c|c|}
\hline $\begin{array}{l}\text { Number of } \\
\text { partners }\end{array}$ & Faisalabad & Karachi & Lahore & Peshawar & Quetta & Rawalpindi & Overall \\
\hline & & & & & & & \\
\hline One & 34.8 & 41.0 & 37.7 & 41.1 & 41.6 & 38.8 & 38.9 \\
\hline Two & 13.3 & 13.7 & 18.9 & 11.6 & 13.9 & 15.3 & 14.3 \\
\hline Three or more & 48.1 & 43.9 & 39.6 & 44.2 & 41.6 & 44.9 & 44.0 \\
\hline Can't recall & 3.8 & 1.4 & 3.8 & 3.2 & 3.0 & 1.0 & 2.7 \\
\hline (N) & (158) & (139) & (106) & (95) & (101) & (98) & (697) \\
\hline
\end{tabular}

A particular area of interest of this study was the sexual behaviors of migrant men to determine whether they are at increased risk for transmission of STIs. Table 6.3 presents non-marital sexual activity among migrants (defined as those who have lived away from their
Table 6.3: Non-marital sexual activity over three time periods, by migrant status ( $>4$ months) (percent)

\begin{tabular}{lrrr}
\hline & \multicolumn{3}{c}{ Migrant status } \\
\cline { 2 - 4 } Time period & Migrant & Non-migrant & p-value \\
\hline Lifetime & 37.2 & 28.2 & 0.004 \\
Last 12 months & 27.1 & 14.3 & $<.000$ \\
Last 3 months & 22.0 & 10.4 & $<.000$ \\
(N) & $(\mathbf{2 1 8 )}$ & $\mathbf{( 2 , 1 8 2 )}$ & $\mathbf{( 2 , 4 0 0 )}$ \\
\hline
\end{tabular}


marital or natal home for more than four months). Migrants did report more sexual activity in their lifetime and in the last 12 and three months than non-migrant men. These differences are statistically significant ( $p$-value <.005).

For all time periods -- lifetime, in the last 12 months and in the last three months -- travelers reported more non-marital sexual activity than non-travelers. The difference between travelers and nontravelers is statistically significant ,as shown in table 6.4 .

Table 6.4: Non-marital sexual activity in 3 time periods, by traveler status ( $>1$ night out of home for work) (percent)

\begin{tabular}{lrrr}
\hline & \multicolumn{3}{c}{ Traveler status } \\
\cline { 2 - 4 } Time period & Traveler & Non-traveler & p-value \\
\hline Lifetime & 39.4 & 27.0 & 0.000 \\
Last 12 months & 20.2 & 14.5 & 0.006 \\
Last 3 months & 15.3 & 10.7 & 0.009 \\
(N) & $\mathbf{( 3 9 1 )}$ & $\mathbf{( 2 , 0 0 9 )}$ & $\mathbf{( 2 , 4 0 0 )}$ \\
\hline
\end{tabular}

These men who spend the night outside of their home for business are more likely to be engaged in non-marital sexual activity. These findings are supported by the literature that argues that mobility due to occupation is a risk factor for STI transmission (Lau et al. 2001). It is possible that these men, because they travel, have greater opportunities to engage in non-marital sex.

\section{Understanding non-marital sexual behaviors: findings from the qualitative research}

In order to develop a better understanding of the sexual behaviors of men we conducted in-depth structured interviews with men who reported having non-marital sex in the last three months. We present here selected general observations from this research that support understandings of non-marital sexual behaviors, particularly as they relate to unpaid sex. Findings from the focus group discussions are presented when appropriate. A more detailed description of the qualitative research can be found in appendix 3.

The qualitative research suggests that non-marital sexual behavior does occur among males 16-45 years and may be more common than previously anticipated. The study findings also reveal that men access all types of sexual partners including other men (both sex workers and men other than sex workers), hijras, and females (both sex workers and females other than sex workers); therefore, a combination of sexual partners are being used. The data suggest that the majority of the respondents had a partner other than a sex worker. 
According to most of the respondents, including those whose last non-marital sex was with a male, their first preference for a sexual partner was a female, but they used a male because of easier availability and greater affordability.

Men felt that sex was a natural desire, which they could not control and were willing, if required, to have sex with whoever was available. But many men were of the opinion that females had been created for having sex.

Unpaid sex. A sizeable majority of the respondents reported having unpaid sex the last time they had intercourse. In some cases sex workers were hired, but most men reported sex with known sexual partners either in the neighborhood or somewhere close by.

"She and I have been neighbors since childhood, but we developed a sexual partnership two years back. I don't pay her anything and she does not expect it either, because she loves me."

IDI, Quetta

To further understand the concept of unpaid sex we draw on the findings of the focus group discussions to provide more clarification.

"An acquaintance of mine is a police officer. He gave me a girl's number. She was a doctor's daughter. He told me that if you keep feeding her you can have sex with her. So it not just poor women but even the well-off girls who do it for fun."

FGD, unmarried 23-year-old, primary educated, Peshawar

Another explanation of unpaid sex was provided by a FGD participant in Peshawar:

\footnotetext{
"Unpaid sex is because girls get fooled by false hopes of marriage."

FGD, married 31-year-old, postgraduate, Peshawar

"Sex on the basis of friendship is common in students."

FGD, unmarried 22-year-old, intermediate pass, Karachi
}

Another way of having unpaid sex with girls was by first enticing them and then blackmailing them (with the threat of disclosure).

"...if you manage to trap a girl you can have sex with her."

FGD, married 42-year-old, intermediate pass, Faisalabad 
Within even steady relationships there is some degree of transaction, which is usually in the form of giving presents or granting favors.

$$
\begin{aligned}
& \text { "In case of unpaid sex (men) facilitate their sexual partners by giving } \\
& \text { gifts like clothes, TV or even a motorcycle." } \\
& \text { FGD, married 35-year-old, matriculate, Faisalabad }
\end{aligned}
$$

Participants were never too sure whether relationships between so-called friends were monogamous or not.

"If a girl has relations with fifty men when you meet her she says that she does not have relations with anyone else."

Married 41-year-old, secondary educated, Karachi

Although the frequency of unpaid sexual activity was not that high, the relationship remained for years and whenever the opportunity arose, such relationships culminated in sexual intercourse. Even married men described having regular extramarital sexual partners they visited whenever they had the opportunity.

Safe places for sex were mentioned as brothel houses, friend's sitting room, or the woman's house. In the case the of sex workers, hotels and motels are also used according to the financial situation of the client.

It was also obvious that there were certain men who only indulged in non-marital sex with sex workers but hired a different woman each time. Only in certain instances did respondents claim to visit some 'favorites' over and over again. The mode of payment was usually cash and ranged from Rs. 40 to 3,000 rupees a night.

The majority of the men said that they had vaginal sex the last time, while less than five respondents had anal sex with a female during their last three sexual encounters. Similarly, oral sex was not that common and many men had not experienced it.

"I find oral sex disgusting. It is a Westernized notion and I find no pleasure in it."

IDI, Quetta

Something that emerged from the in-depth interviews and is also endorsed in the FGD findings is the sense of guilt among men regarding illicit or extramarital sex. Their responses reflect a sense of wrongdoing in having non-marital sex, and many said they planned never to do it again. It was ironic that a few respondents first described their 
non-marital sexual encounters and then said that society was being ruined due to cable television and the Internet.

"We should practice proper Islam and stop watching cable and internet. Everything will be fine if we are a truly Islamic country."

IDI, Lahore

Sexual partners were usually availed during work or encounters arranged through friends. Mobile phones were mentioned as a major source for finding sexual partners. Men said that they got in touch with their sexual partners, both paid and unpaid, through mobile phones.

"Nowadays it is very easy to access a sexual partner or get alcohol or drugs. You just make a phone call and services will be available. It can be through a pimp or even directly. Similarly, here alcohol can also reach your doorsteps in no time."

IDI, Faisalabad

It was also a common practice for friends to arrange for a sex worker and mutually share the experience. Usually group sex was not practiced, but the FSW was shared one-by-one.

Reasons given by the respondents for engaging in illicit or extramarital sex were first biological need followed by desire for a variety of sexual partners. Married men mostly said they got bored with their wives and needed variety.

"It is a natural desire to have sex. Men have this need and sex definitely relaxes one. A man needs to change his taste after a while and the wife starts getting stale as a sexual partner."

IDI participant, Karachi

Some men also confessed that they felt sexually inhibited with their spouses and could not do certain sexual acts with them.

"With one's wife a person can only do conventional sex. With a sex worker you can do anything.

IDI, Peshawar

A high number of respondents felt that there was no advantage to having a temporary relationship, and such relationships definitely exposed them to STIs, but they were also more exciting. 
"There is much more pleasure in illicit sex. It always excites me more and that is why I go for it over and over again."

IDI, Rawalpindi

Most men also agreed that their sexual partners probably had other sexual partners as well, which was of course the case for paid sex. But even in the case of unpaid sex, some men were not sure whether their sexual partner had other partners than them.

"I can't really say whether she has sex with others as I don't keep a tag on her. I visit her once in a month or two whenever we get a chance."

IDI, Lahore

"Of course she has other sexual partners as well because after all it is her profession to sell sex which makes me vulnerable to STIs."

IDI, Karachi

\section{Condom use}

Condom use plays dual beneficial roles: if used consistently, it is an effective contraceptive method and it also prevents transmission of STIs, including HIV. Promotion of condom use, whenever culturally appropriate, is one of the main components of any HIV prevention program.

Ever use. Regardless of marital status, all respondents in the structured questionnaire who reported ever having sex were asked about condom use during their lifetime. Of the 68 percent of respondents $(n=1,643)$ who had ever had sexual intercourse, 37 percent $(n=604)$ reported having used a condom at least once in their lifetime (figure 6.8). The highest reported proportions of ever-use of condoms was in Rawalpindi (44 percent), followed by Faisalabad (40 percent), Lahore (40 percent), Peshawar (38 percent), Quetta (33 percent), and Karachi (27 percent). Of the 1,643 men reporting any sexual experience, 1,261 were married and 382 were unmarried. Of those who had ever used a condom, 81 percent $(n=493)$ were married. 
Figure 6.8: Percentage distribution of men who ever used a condom, by city

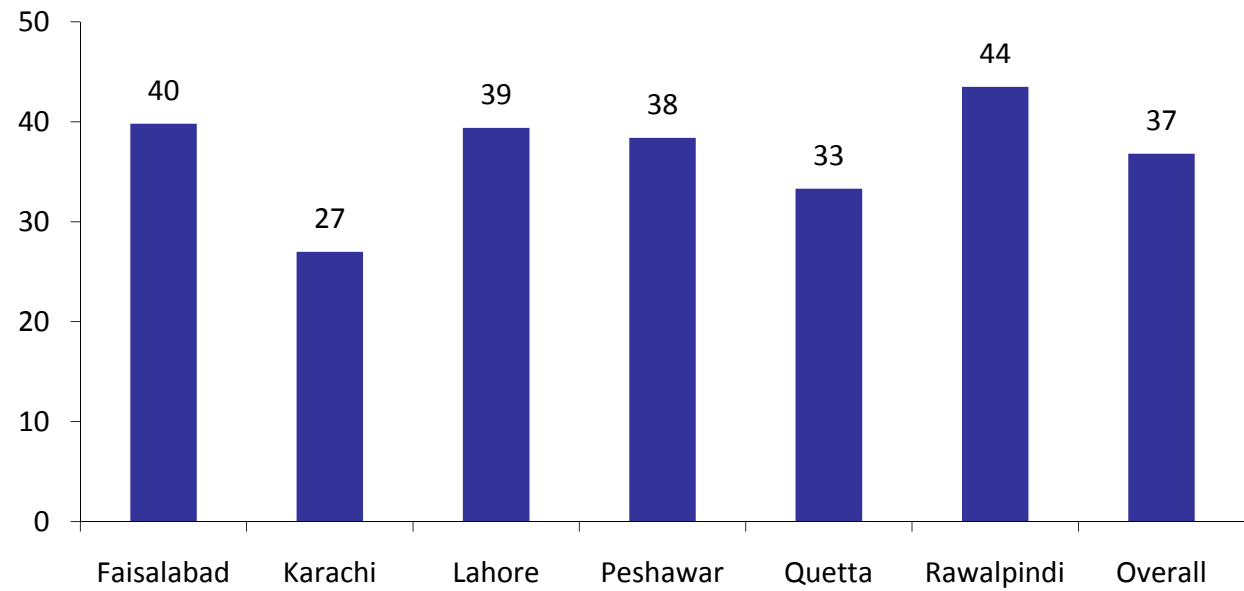

Respondents were asked about their condom use during their last non-marital sexual intercourse (figure 6.9). As this question refers to the most recent sexual act, it minimizes recall bias. Among all respondents, condom use at last non-marital sex was 16 percent. In all cities, except Rawalpindi where it was 25 percent, less than one-fifth (20 percent) of the male respondents reported condom use during last non-marital sexual intercourse.

Figure 6.9: Percentage distribution of men who used a condom during their last nonmarital sexual intercourse, by city

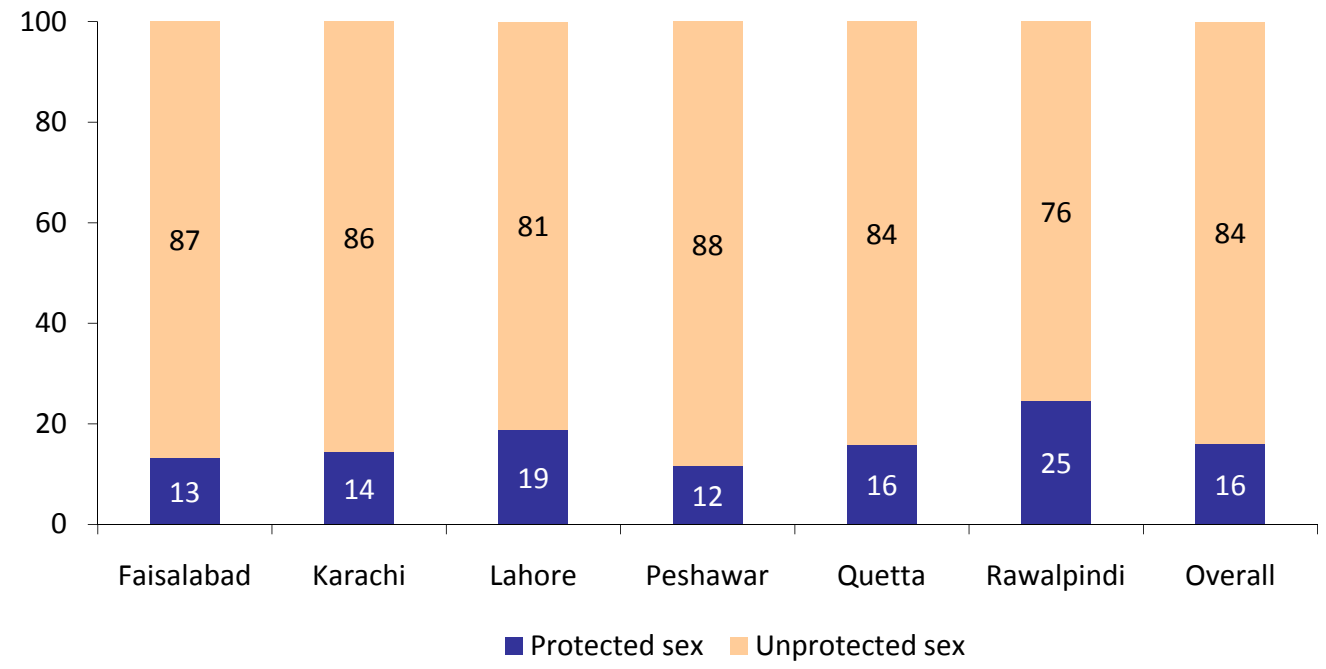


Condom use during last sexual intercourse and background characteristics. To better identify the men who used condoms, condom use at last sexual intercourse was analyzed based on age, education, marital status, and migration status. The results are described below.

Age. Out of the total sample, 697 respondents reported that their last sexual encounter was non-marital. When examining condom use by age among these men, we find a higher proportion using condoms in the age group 21-30 years (figure 6.10). In Rawalpindi there was a higher percentage of condom use among the age group 21-30 years; in Karachi, use in the age group 16-20 years was low compared to the other cities. (For city wise details, see appendix 1.)

Figure 6.10: Percentage distribution of men who used condom during last non-marital intercourse, by age

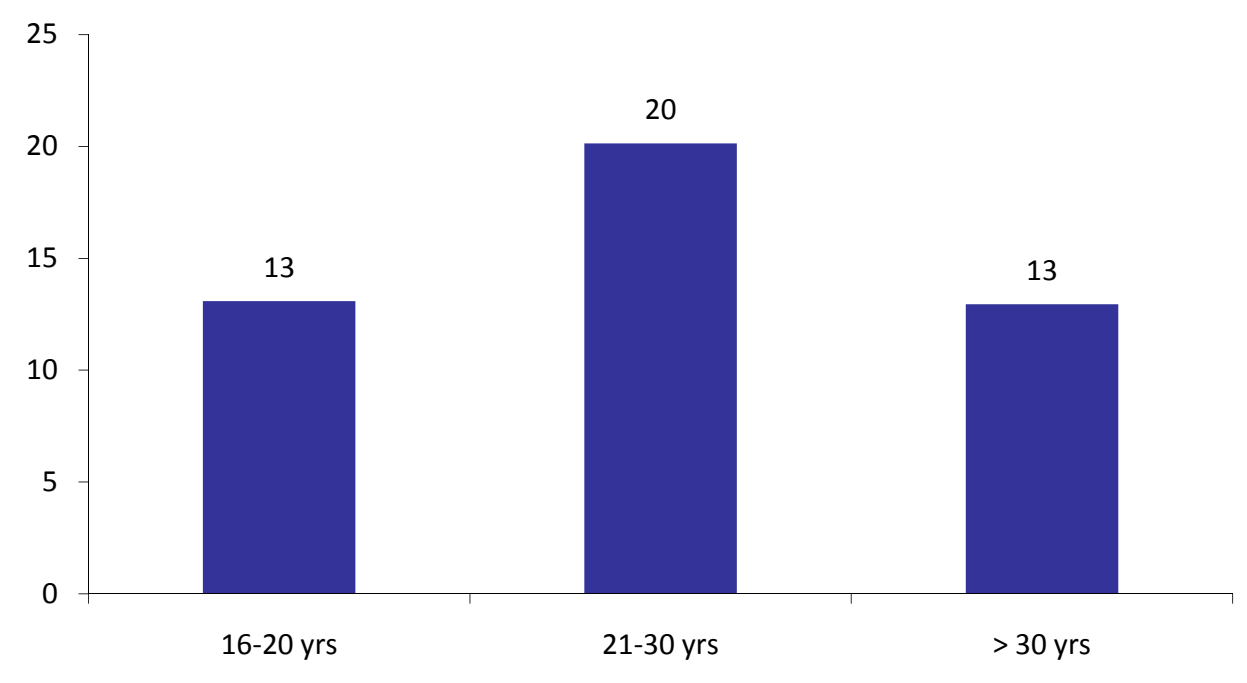

Education. The international literature suggests that educational levels are correlated with condom use (La Garde et al. 2001). Our study found that condom use at last sexual intercourse was higher among men who had greater than secondary education. Current results are presented in figure 6.11. (For city wise details, see appendix 1.) 
Figure 6.11: Percentage distribution of men who used a condom during last non-marital intercourse, by educational level

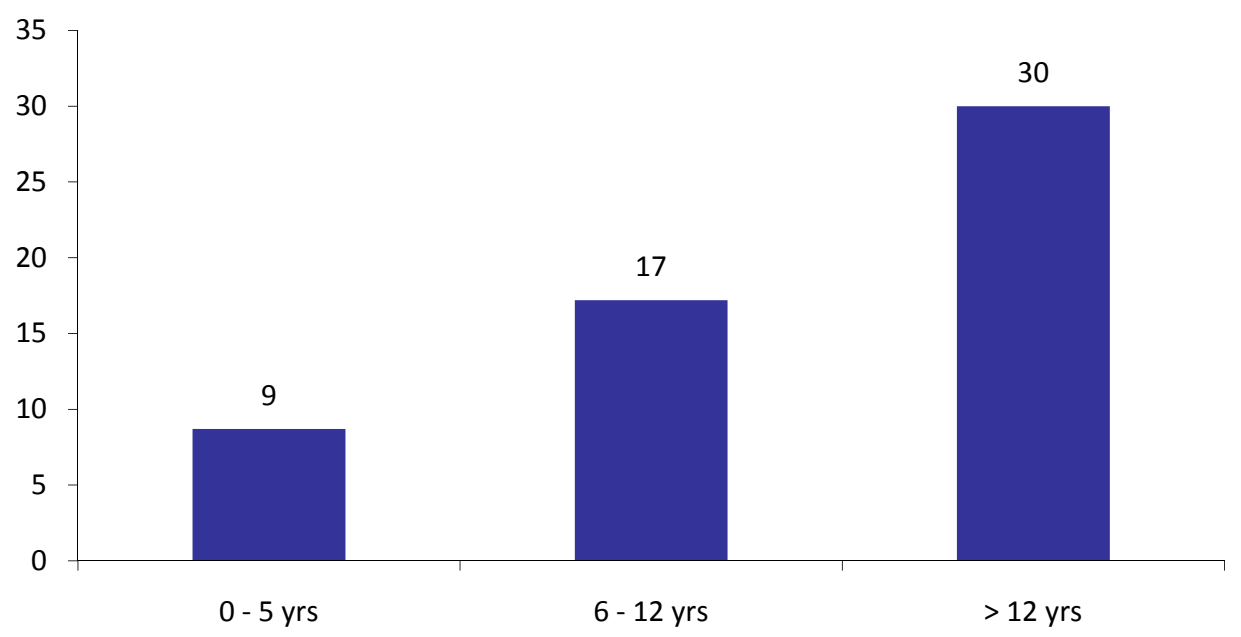

Marital status. Survey participants were asked about condom use during their last sexual intercourse, irrespective of their marital status (figure 6.12). Across all cities, except Karachi, currently unmarried respondents reported using a condom at their last sexual intercourse more often than married men. Rawalpindi had the highest proportion of currently unmarried respondents reporting condom use, followed by Lahore. In Karachi, the proportion of men who used a condom at their last sexual intercourse was similar for both married and non-married men (15 percent). (For city wise details, see appendix 1.)

Figure 6.12: Percentage distribution of men who used a condom during last non-marital intercourse, by marital status

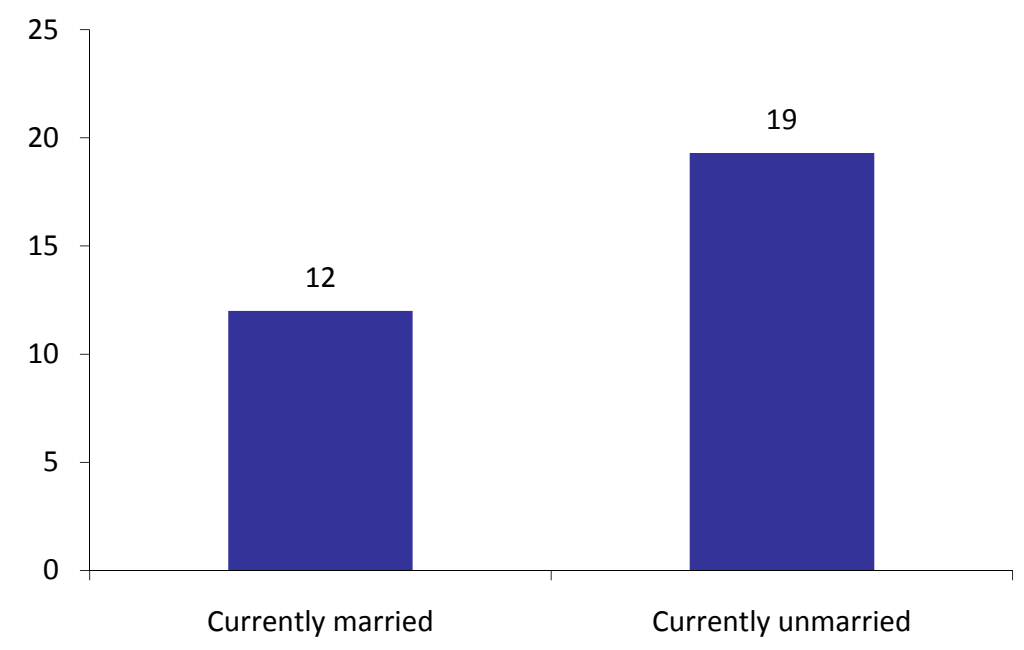


Migration status. In this study, migrants were defined as those living away from their marital or natal home for greater than four months; travelers were defined as those men who had spent at least one night outside of their marital or natal home because of their job. When examining condom use at last non-marital sexual experience by migrant status, the travelers were more likely to use a condom (26 percent) than the migrants (9 percent) (figure 6.13). Use of condom in travelers was almost similar (about 15 percent) in Karachi, Lahore, Peshawar, and Quetta, while in Rawalpindi it was higher (43 percent). In Faisalabad, condom use was comparatively low for both travelers and non-travelers. Overall, non-migrants and non-travelers were most likely (65 percent) to use a condom at last non-marital sexual experience. (For city wise details, see appendix 1.)

Figure 6.13: Percentage distribution of respondents according to condom use at last non-marital sexual intercourse, by migrant status

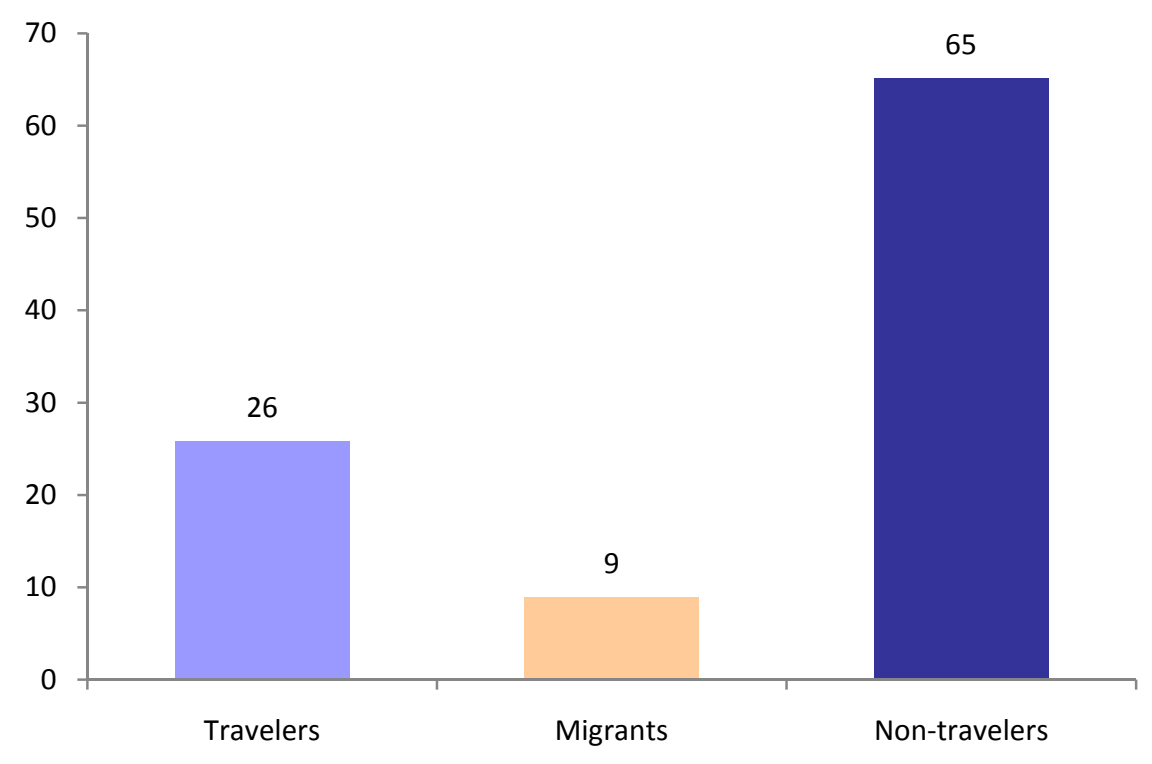

Reasons for using condoms. Respondents were asked the reasons they used a condom with their most recent non-marital sexual partner. The results are reported in table 6.5 by type of partner for the overall population $(n=130)$. The types of non-marital partners include: female other than sex worker (FOSW) (female/friend); male other than sex worker (MOSW); male sex worker (MSW); and hijra. Respondents were allowed to share more than one reason, therefore the column percentages add to more than 100 percent.

The numbers of men who used a condom during their most recent sexual intercourse 
with a non-marital sexual partner are small, and so caution should be applied when interpreting the data. The most common reasons given for using a condom with a female other than sex worker was to avoid pregnancy, followed by avoiding getting a disease. In comparison, the most common reason for using a condom with a female sex worker was to avoid getting a disease.

Reasons for not using condoms. Those men who did not use a condom at their last nonmarital sex were asked their reasons for nonuse. Again, multiple responses were possible. The results are shown in the table 6.6.

The majority of respondents said that they did not think of using a condom with a female sex worker(FSW) as well as with a female other than sex worker. Information from the FGDs in the qualitative research suggests that these sexual acts are mostly spontaneous and/or carried out surreptitiously and with little preparation.
Table 6.5: Percentage distribution of respondents by reason for condom use last time and partner

Partner and reason Percent

Avoid pregnancy 81.3

Avoid giving a disease $\quad 28.0$

Avoid getting a disease $\quad 41.3$

Partner's wish $\quad 2.7$

Other 1.3

(N)

FSW

Avoid pregnancy 29.3

Avoid giving a disease $\quad 19.5$

Avoid getting a disease $\quad 65.9$

Partner's wish 7.3

Other 2.1

(N)

MOSW

Avoid giving a disease $\quad 25.0$

Avoid getting a disease $\quad 50.0$

Other 25.0

(N) (4)

MSW $^{\mathrm{a}} 0.0^{\mathrm{a}}$

Hijra

Avoid getting a disease $\quad 66.7$

Other 33.3

(N) (3)

${ }^{a}$ No men reported using a condom with a male sex worker.

In the case of sex with males, the main reason

for not using a condom was that respondents felt it was not necessary suggesting that respondents consider condoms to be a method of family planning rather than protection against sexually transmitted diseases. 
Table 6.6: Percentage distribution of respondents by reasons for not using condom last time and type of partner

\begin{tabular}{|c|c|c|c|}
\hline Partner and reason & Percent & Partner and reason & Percent \\
\hline FOSW & & MSW (continued) & \\
\hline Not available & 9.4 & Didn't think of it & 22.9 \\
\hline Partner objected & 1.5 & Reduces pleasure & 14.6 \\
\hline Don't like them & 13.8 & Other & 22.9 \\
\hline Contraceptives & 2.6 & (N) & (48) \\
\hline It was not necessary & 20.9 & MOSW & \\
\hline Didn't think of it & 31.5 & Not available & 5.3 \\
\hline Reduces pleasure & 9.7 & Partner objected & 0.8 \\
\hline Other & 22.6 & Don't like them & 16.8 \\
\hline (N) & (340) & Contraceptives & 0.8 \\
\hline FSW & & It was not necessary & 33.6 \\
\hline Not available & 9.4 & Didn't think of it & 28.2 \\
\hline Partner objected & 0.5 & Reduces pleasure & 6.9 \\
\hline Don't like them & 20.7 & Other & 19.1 \\
\hline Contraceptives & 0.5 & (N) & (131) \\
\hline It was not necessary & 15.8 & Hijra & \\
\hline Didn't think of it & 36.5 & Not available & 3.3 \\
\hline Reduces pleasure & 9.9 & Don't like them & 20.0 \\
\hline Other & 17.7 & It was not necessary & 30.0 \\
\hline (N) & (203) & Didn't think of it & 20.0 \\
\hline MSW & & Reduces pleasure & 10.0 \\
\hline Not available & 4.2 & Other & 26.7 \\
\hline Don't like them & 12.5 & (N) & (30) \\
\hline It was not necessary & 29.2 & & \\
\hline
\end{tabular}

Condom use with wife and extramarital partners. A cross-tabulation examining the level of condom use with wives and other partners among married men was carried out. Despite the low numbers, this information is important to report, as not using a condom with both wives and extramarital partners is likely to transmit infection and is an important behavior in the bridging from high-risk groups to the general population.

The data (shown in appendix 1) show a high proportion of men who did not use condoms either with FSWs or with their wives (around 85 percent for all cities). In Karachi and Lahore, only one-fifth (20 percent) of men used a condom with both FOSWs and their wives, and in Faisalabad the proportion increased to 43 percent $(n=3)$. In Rawalpindi, the proportion of men who used condoms with both FOSWs and their wives was 40 percent $(n=2)$, and in Quetta it was 60 percent $(n=3)$.

Overall in the six cities, condom use with FSWs and wives was very low (25 percent). There was no respondent who reported condom use with an FSW in Peshawar, and in Karachi and Lahore none of those who used condoms with FSWs reported current condom use with their wives. In Quetta and Karachi, a high proportion of the 
respondents (95 percent) did not use a condom with FSWs or their wives, followed by Rawalpindi (88 percent) and Lahore (81 percent).

Table 6.7: Percentage of condom use with wife and extramarital partner at last sexual intercourse

\begin{tabular}{lrrr}
\hline Partner & Condom used with wife & Condom not used with wife & (N) \\
\hline Condom used w/FOSW & 33.3 & 66.7 & $\mathbf{3 0}$ \\
Condom not used w/FOSW & 14.6 & 85.4 & $\mathbf{1 , 2 1 5}$ \\
Condom used w/FSW & 25.0 & 75.0 & $\mathbf{1 2}$ \\
Condom not used w/FSW & 14.9 & 85.1 & $\mathbf{1 , 2 3 3}$ \\
Condom used w/male $^{\mathrm{a}}$ & 0.0 & 100.0 & $\mathbf{1}$ \\
Condom not used w/ male $^{\mathrm{a}}$ & 15.0 & 85.0 & $\mathbf{1 , 2 4 4}$ \\
\hline
\end{tabular}

a "Male" includes male friends, male sex workers, and hijras.

Using condoms with male partners was almost nonexistent across the study population. In all cities, except Faisalabad and Rawalpindi, more than 85 percent of the married respondents reported not using a condom with a male partner or their wives.

Frequency of condom use in non-marital sexual relationship over the last 12 months by type of partner. Respondents were asked about their frequency of condom use by type of partner. This information was reported on condom use over the last twelve months, allowing for assessment of consistency in condom use in nonmarital sexual relationships.

Figure 6.14 shows that out of the total men who reported non-marital sexual intercourse during the last 12 months $(n=370)$, more than two-thirds (68 percent) had 'never' used a condom irrespective of their sexual partner, while a small proportion (5 percent) reported using a condom 'often'. Ten percent of respondents reported using a condom 'every time', and those who used a condom 'sometimes' were slightly less than one-fifth (17 percent). Table 6.8 describes the details of frequency of condom use by type of partner in the last 12 months for the overall study population. (For city wise distribution see appendix 1). 
Figure 6.14: Frequency of condom use during the last 12 months in non-marital sexual relations

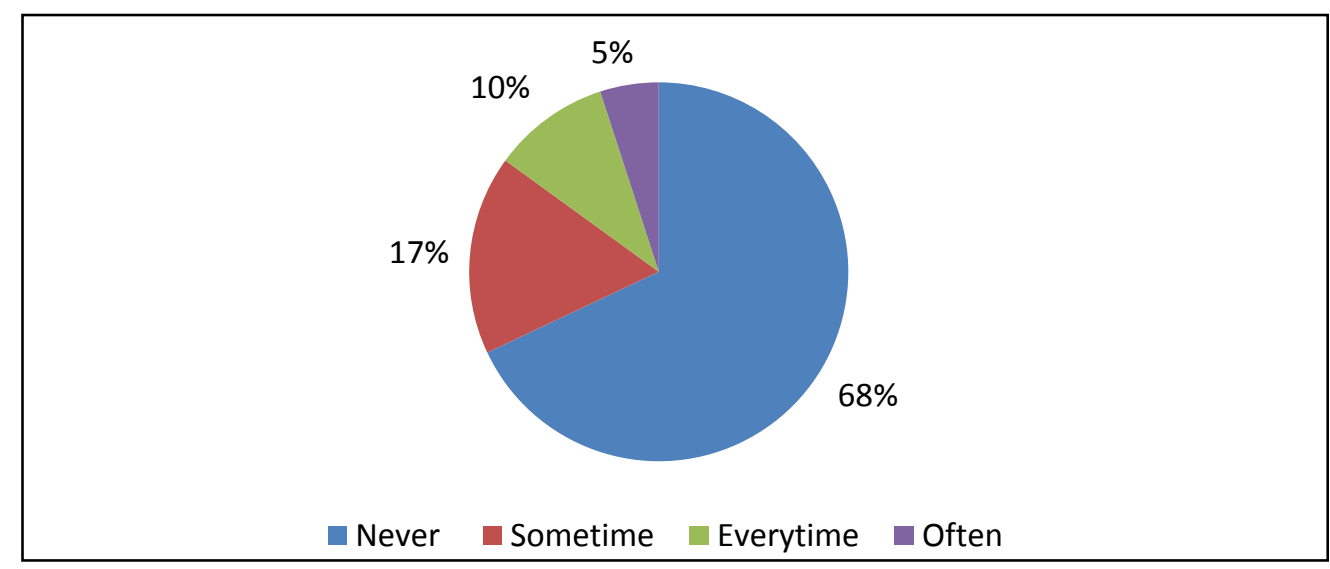

Table 6.8: Frequency of condom use in last 12 months, by type of partner

\begin{tabular}{|c|c|c|c|}
\hline Partner and frequency & Percent & Partner and frequency & Percent \\
\hline FOSW & & MSW (continued) & \\
\hline Every time & 10.5 & Never & 100.0 \\
\hline Often & 5.0 & (N) & (32) \\
\hline Sometimes & 18.6 & Mosw & \\
\hline Never & 65.9 & Every time & 0.0 \\
\hline$(\mathrm{N})$ & (220) & Often & 0.0 \\
\hline FSW & & Sometimes & 5.6 \\
\hline Every time & 10.1 & Never & 94.4 \\
\hline Often & 7.2 & $(\mathrm{~N})$ & (71) \\
\hline Sometimes & 15.8 & Hijra & \\
\hline Never & 66.9 & Every time & 5.9 \\
\hline (N) & (139) & Often & 0.0 \\
\hline MSW & & Sometimes & 5.9 \\
\hline Every time & 0.0 & Never & 88.2 \\
\hline Often & 0.0 & $(\mathrm{~N})$ & (17) \\
\hline Sometimes & 0.0 & & \\
\hline
\end{tabular}

Condom use with Female Other than Sex Worker (FOSW). Out of the 220 respondents who reported non-marital sexual intercourse in the last 12 months with FOSWs, two-thirds reported never using a condom. Across the six cities, condom use 'every time' with FOSWs was the highest in Peshawar (19 percent), followed by Quetta (16 percent). In Karachi, most respondents (83 percent) never used a condom while having sex with a female who was not a sex worker. In Rawalpindi, one-half of all men 
who had sex with FOSWs reported never using a condom with them. This was the lowest proportion when compared to other cities.

Condom use with female sex workers (FSW). Across all six cities, 139 men reported sexual intercourse with FSWs during the last 12 months. Of the 139 men, 67 percent reported never using a condom with FSWs during the last 12 months. Ten percent of the 139 men who reported sexual intercourse with FSWs reported using a condom 'every time'. The highest percentage of those who never used a condom during intercourse with FSWs was in Karachi (79 percent), but respondents from the remaining five cities who never used a condom were similar.

Condom use with males (MSW, MOSW and hijra). Overall in the study population, condom use with male partners was low as with female sex workers. Out of the total number of men who had sex with other men (32 with MSWs; 71 with MOSWs; and 17 with hijras), more than 90 percent reported never using a condom. With MSWs, all men replied that they had never used a condom. With MOSWs, most men never used a condom either, except in Faisalabad (where two out of the 18 men 'sometimes' used a condom), Quetta (one out of 16 men reported 'sometimes' using a condom), and Rawalpindi (one out of nine men reported 'sometimes' using a condom). In the entire study population, 17 respondents reported sexual activity with a hijra. Of these, 88 percent reported that they never used a condom with a hijra.

Much of the qualitative data confirm the quantitative results. In the in-depth interviews, although almost all respondents felt that they were vulnerable to STIs due to their non-marital sexual activities, only 16 percent had actually used a condom during their last sexual encounter. Awareness about condoms was quite widespread, but many still perceived it as a means to prevent pregnancy rather than as protection from STIs. Most men also said that they did not enjoy sex with a condom because they felt that it interfered with their sexual pleasure. In the qualitative research, many men said that using a condom did not cross their minds or there was no time to use a condom due to sex being an unplanned activity. Some men who did not use a condom regularly or every time, did say they would use them based on their availability or the situation at that moment. 


\section{Non-sexual practices and behaviors Injection practices and blood transfusions}

Unsafe injection practices are an important risk factor in the spread of HIV infection. Several reports suggest that medical injections are overused in Pakistan (Raglow et al. 2001), and that reuse of injection equipment meant for single-use is also common (Luby et al. 1997). It has been estimated that the rate of medical injection use in Pakistan is around 4.5 injections per capita per year (Family Health International 2007). Patients receive injections from doctors and other medical practitioners in Pakistan frequently, and often without proper safety precautions. Patients may be aware of both the positive and negative aspects of injections, but they are likely to do whatever the doctor suggests (Raglow et al. 2001). The findings from this survey contribute to existing data with community-based evidence from a considerable sample size.

Table 6.9 presents the history of the respondents' medical injection use during the last one year. No study participant reported injecting drug use. Across all of the cities, 79 percent of respondents had received at least one injection during the last year. The number of injections reported ranged from 1 to 87, with the mean number being 6.1 injections. Forty percent of all respondents had received between 1 and 5 injections. However, nearly one-quarter of all respondents had received

$\begin{aligned} & \text { Table 6.9: History of medical } \\
& \text { injections in the past year }\end{aligned}$
\begin{tabular}{lrr}
\hline $\begin{array}{l}\text { Number of } \\
\text { injections }\end{array}$ & $\begin{array}{r}\text { Number of } \\
\text { respondents }\end{array}$ & Percent \\
\hline 0 & 515 & 21.5 \\
1 to 5 & 962 & 40.1 \\
6 to 10 & 247 & 10.3 \\
11 to 20 & 104 & 4.3 \\
21 to 87 & 572 & 23.8 \\
Total & $\mathbf{2 , 4 0 0}$ & $\mathbf{1 0 0 . 0}$ \\
\hline Mean=2.0 & & \\
\hline
\end{tabular}
between 21-87 injections. This high number of reported injections in one year could be related to the high number of injections required for treatment of hepatitis $C$.

Further analysis illustrates that among those respondents who received injections during the last one year, 12 percent reported they were not sure whether the syringe was new or used because it was not opened in front of them; the remaining respondents ( 88 percent) reported that the syringe was new and opened in front of them.

Overall, only 3 percent of the survey respondents reported having a blood transfusion one or more times (figure 6.15). Among these, 64 percent reported that they thought the blood they received had been routinely screened for disease. 
Figure 6.15: Self-reported blood screening before blood transfusion

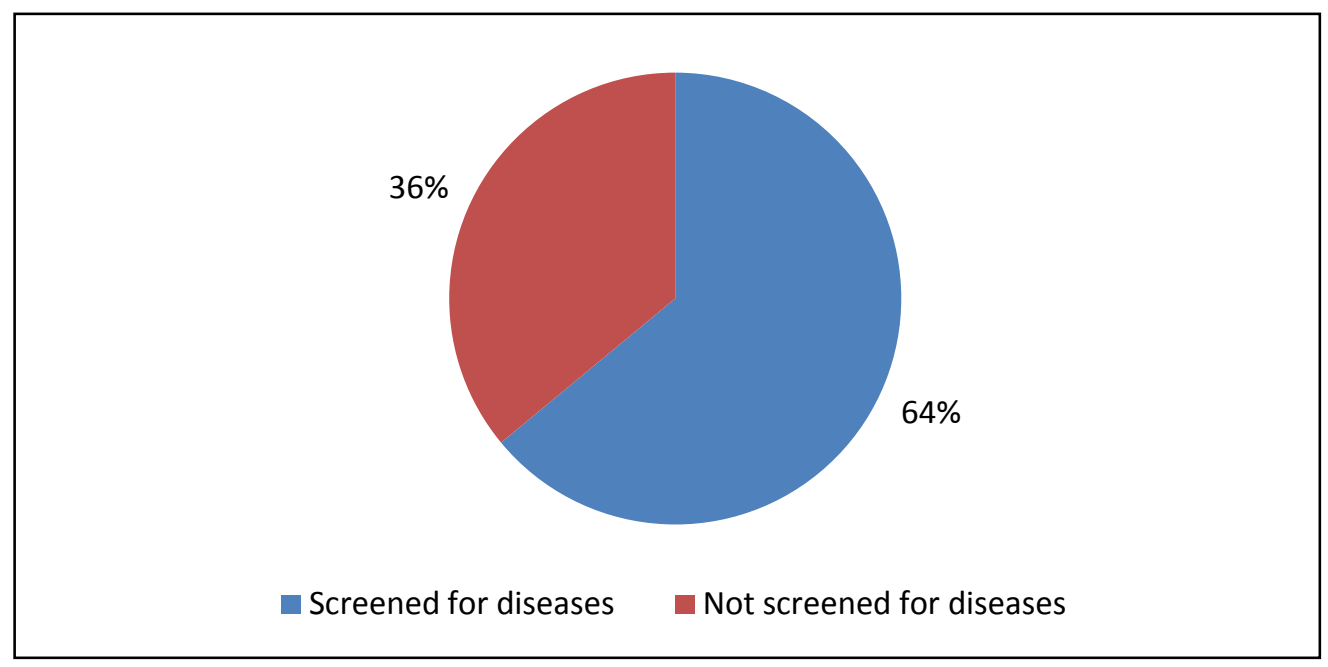

Tooth extraction has the potential to Table 6.10: Tooth extraction, by type of increase transmission of blood-borne service provider infections. Almost one-third of the total sample (29 percent) had ever had a tooth extracted, and one-quarter of these had done so with an unqualified dentist (table

\begin{tabular}{lrr}
\hline Provider & Number & Percent \\
\hline Dentist & 526 & 75.0 \\
MBBS doctor & 111 & 15.8 \\
Other & 64 & 9.1 \\
Total & $\mathbf{7 0 1}$ & $\mathbf{1 0 0 . 0}$ \\
\hline
\end{tabular}
6.10).

\section{Intoxicants}

Intoxicant use can increase sexual risk-taking behaviors (Adrian 2006). Overall, about one-third of the respondents (31 percent, $n=736$ ) reported ever using any intoxicant, which included alcohol, stimulants (naswar, and gutka/pan) and narcotics (e.g., charas, heroin, biri, bhang [cannabis]). City wise analysis shows that self-reported ever-use of intoxicants ranged from 24 percent to 43 percent, with lowest use reported in Rawalpindi and highest in Karachi. Current use of intoxicants was reported by 7 percent of the respondents.

Chi-square analysis was carried out to test the association between those respondents using intoxicants and those who had engaged in non-marital sex in the last 12 months (the p-value was .000). As figure 6.16 illustrates, those who had used intoxicants in the last 12 months were also more likely to have engaged in pre- or extramarital sex during the same period. 
Figure 6.16: Intoxicant use among those having non-marital sex in the last 12 months

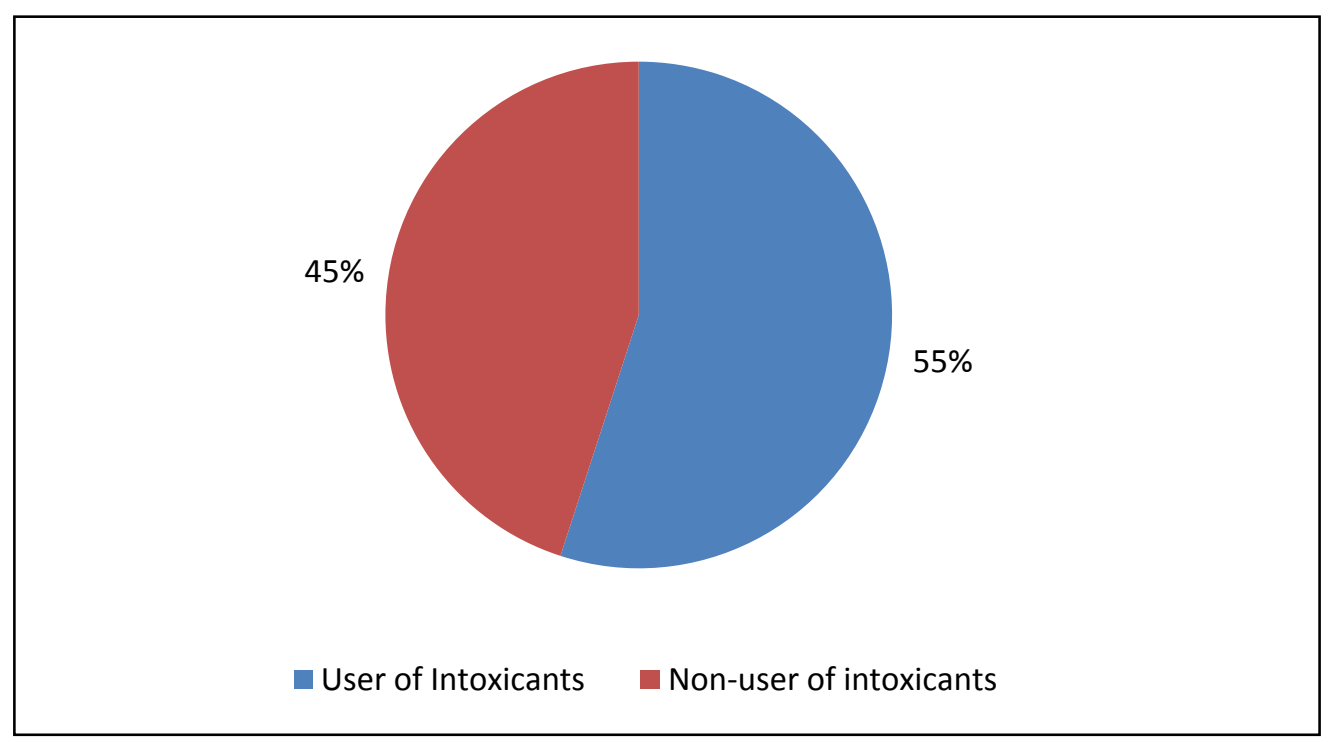

\section{Personal hygiene}

Hygiene interventions to reduce the risk of acquiring STIs date back to the First and Second World Wars. Evidence showed that servicemen who had been exposed to soap and water prophylaxis had decreased incidence of genital ulcer disease (Franco 1988). Penile cancer and cervical cancer in females has also been linked to male genital hygiene (Brinton 1991). Recent evidence also recommends the need for improving personal hygiene norms ( $\mathrm{O}^{\prime}$ Farrell et al. 2006). In this section, we describe the genital hygiene practices in terms of washing the genital area after urination, and prior to and after having sex.

Overall, 72 percent of all respondents $(n=1,728)$ reported washing their genital area every time after passing urine, while 20 percent often washed and 7 percent seldom washed. Only 18 respondents reportedly never washed. The highest number of responses was reported in Faisalabad and Peshawar (81 percent each), followed by Lahore (79 percent), Karachi (71 percent), and Rawalpindi (62 percent). The fewest were in Quetta, where only 55 percent reported washing every time after urination. This could be linked to the absence of tapped water and toilet facilities in the home; in Quetta, only one-quarter of households had an attached toilet to the room, and only 60 percent of households had tap water. 
Table 6.11: Percentage distribution of respondents according to personal hygiene practices, by city

\begin{tabular}{|c|c|c|c|c|c|c|c|}
\hline Practice & Faisalabad & Karachi & Lahore & Peshawar & Quetta & Rawalpindi & Overall \\
\hline \multicolumn{8}{|c|}{ Wash genital area after urination } \\
\hline Every time & 80.7 & 71.3 & 78.8 & 80.2 & 55.5 & 62.2 & 71.5 \\
\hline Often & 12.8 & 16.5 & 17.4 & 8.8 & 35.0 & 31.9 & 20.3 \\
\hline Sometimes & 5.5 & 11.3 & 3.3 & 9.8 & 8.8 & 6.0 & 7.4 \\
\hline Never & 1.0 & 1.0 & 0.5 & 1.3 & 0.8 & 0.0 & 0.8 \\
\hline (N) & (400) & (400) & (400) & (400) & (400) & (400) & $(2,400)$ \\
\hline \multicolumn{8}{|c|}{ Wash genital area before having sex } \\
\hline Yes & 32.7 & 41.4 & 23.6 & 41.7 & 31.1 & 23.5 & 32.5 \\
\hline No & 67.3 & 58.6 & 76.4 & 58.3 & 68.9 & 76.5 & 67.5 \\
\hline$(\mathrm{N})^{\star}$ & (299) & (274) & (282) & (271) & (285) & (232) & $(1,643)$ \\
\hline \multicolumn{8}{|c|}{ Wash genital area after having sex } \\
\hline Yes & 94.9 & 96.7 & 93.9 & 93.6 & 95.4 & 89.1 & 94.1 \\
\hline No & 5.1 & 3.3 & 6.1 & 6.4 & 4.6 & 10.9 & 5.9 \\
\hline$(\mathrm{N})^{*}$ & (299) & (274) & (282) & (271) & (285) & (232) & $(1,643)$ \\
\hline
\end{tabular}

*Includes only respondents who report sexual experience.

The data in table 6.11 show that the practice of washing the genital area prior to having sex was not widespread, with one-third (33 percent) of the total respondents in all six cities reporting such a practice.

Washing after sex was more common: 94 percent of all respondents who had sexual experience reported such a practice. The highest number was in Karachi, where 97 percent respondents washed after having sex, and the lowest percentage was in Rawalpindi (still a relatively high 89 percent). The presence or absence of an attached toilet in the house and tapped water does not seem to influence this practice.

Respondents were asked to give the reasons for washing themselves after having sex (multiple responses were allowed). The majority cited personal hygiene as the main reason (76 percent). In Karachi, 85 percent of the respondents said they washed because of personal hygiene, while the lowest response for this category was in Quetta (54 percent). Fifty percent of all respondents cited religious injunctions as a reason for washing after sex. The highest percentage of responses citing religious injunction as a reason was in Faisalabad (66 percent), and the least were in Karachi and Rawalpindi (37 percent each). One-fifth of all respondents ( 20 percent) said that avoiding diseases was a reason for washing after sex.

Bivariate analysis found no substantial difference in the genital-hygiene practices of the subset of men who had non-marital sex in the last three months as compared to the overall sample, suggesting that hygienic practices remain the same irrespective of sexual partner. 


\section{Exposure to pornography}

Sixty percent of the total respondents admitted ever having seen pornographic material in their lifetime (table 6.12). Of these men, 67 percent mentioned viewing pornography on videos or CDs; 16 percent reported watching pornography on the Internet, while 5 percent had viewed printed materials (table 6.13).

Table 6.14 shows that among married men, 50 percent reported having ever seen pornographic materials. Among the unmarried, 71 percent had seen pornographic materials.

Among those reporting non-marital sex ever during their lifetime, 81 percent had seen pornographic materials. Among these men ( $n=568), 94$ percent admitted having viewed pornographic videos or CDs (see table 6.15).

The qualitative research suggests that nonmarital sexual behavior especially among young males is influenced by exposure to pornography though cable $\mathrm{TV}$, the Internet, and the easy availability of videos and CDs. The qualitative data suggests that these materials have some influence on sexual norms or practices such as group sex and preferences for oral and anal sex. The type of pornographic material viewed by those who had ever had non-marital sex is shown in table 6.15 .
Table 6.12: Respondents who had ever viewed pornographic material

\begin{tabular}{lrr}
\hline Response & Percent & N \\
\hline Did view & 60.4 & 1,449 \\
Did not view & 39.5 & 949 \\
No Information & 0.1 & 2 \\
\hline
\end{tabular}

Table 6.13: Type of pornographic material ever viewed

\begin{tabular}{lrr}
\hline Pornographic & Percent & N \\
\hline material & 4.9 & 92 \\
Vinted material & 67.2 & 1,271 \\
Playing cards & 7.7 & 145 \\
Internet & 15.9 & 301 \\
Other & 4.3 & 82 \\
Total & na & $\mathbf{1 , 4 4 9}$ \\
\hline
\end{tabular}

${ }^{*}$ na $=$ not applicable; multiple responses were allowed.

Table 6.14: Percentage who viewed pornographic material, by marital status

\begin{tabular}{lll}
\hline Response & Married & $\begin{array}{l}\text { Not } \\
\text { married }\end{array}$ \\
\hline Did view & 50.2 & 71.2 \\
Did not view & 49.3 & 28.7 \\
(N) & $\mathbf{1 , 2 4 5}$ & $\mathbf{6 9 7}$ \\
\hline
\end{tabular}

Table 6.15: Type of pornographic material ever viewed by those who ever had non-marital sex

\begin{tabular}{lrr}
\hline Pornographic & & \\
material & Percent & N \\
\hline Printed material & 7.6 & 43 \\
Video/CD & 94.0 & 534 \\
Playing cards & 12.7 & 72 \\
Internet & 15.8 & 90 \\
Other & 4.2 & 24 \\
(N) & na & $\mathbf{5 6 8}$ \\
\hline
\end{tabular}




\section{Chapter summary}

The data on behaviors related to STI transmission and STI prevalence provides important information about sexual behaviors of men in the general population. Out of the study population, 31 percent were virgins, 42 percent had their first sexual intercourse with their wife, and 27 percent had engaged in premarital sex. The premarital sexual debut was most commonly with a female other than sex worker (53 percent); female sex worker (28 percent); male other than sex worker and hijra (17 percent); and male sex worker ( 3 percent). Sexual debut was in the younger ages with 44 percent of premarital sexual debut occurring between the ages of 16 and 20 years.

When examining both premarital and extramarital sexual behavior (non-marital sex), 29 percent of the population had engaged in non-marital sex in their lifetime. Thirteen percent of this was extramarital sex and 16 percent was premarital sex. Those men who engaged in non-marital sex also reported a number of lifetime non-marital partners. Forty-four percent had three or more lifetime non-marital partners. Migrants and travelers reported more non-marital sex throughout the three reporting periods (lifetime, last 12 months, and last three months).

The results on condom use suggest that this is not a common practice among urban men. Thirty-seven percent reported ever using a condom and 16 percent reported using a condom at last non-marital sex. Those with higher levels of education did report more condom use. Non-migrants and non-travelers also reported more condom use which supports the theory that migrant and mobile men are at greater risk for transmitting STIs and HIV. When men were asked their reasons for using a condom by type of partner, prevention of pregnancy was mentioned for both female other than sex worker (81 percent) and female sex worker (29 percent). Prevention of getting a disease was less frequently reported. An important finding in terms of the future of STI transmission is the low rates of condom use with both wife and extramarital partner. Only 16 percent of men reported using a condom at their last non-marital sex.

In terms of non-sexual behaviors, the results are less clear but may suggest some areas of protection against STI transmission. Ninety-four percent of men report washing their genitals after sex and 31 percent reported ever use of an intoxicant. There was no respondent who reported intravenous drug use. However, among men reporting nonmarital sex in the last 12 months, 55 percent reported intoxicant use. Among those reporting non-marital sex ever during their lifetime, 81 percent had seen pornographic materials. 



\section{THE BRIDGING POPULATION}

The bridging population is understood to be the population that transmits HIV/AIDS and other STIs because of their contact with the high-risk population (i.e., sex workers) and sexual contact with the general population (wife or regular female sexual partner) (Lowndes et al. 2002; Gorbach et al. 2000). How this bridging population is identified and defined can be based on prevalence data (if known) or through the identification of risk behaviors suspected to create the bridge from the high-risk groups to the general population. The review of the international literature suggests that the bridging population will vary from setting to setting depending on the cultural, epidemiological, and economic environment of the particular context.

Identifying the bridging population, therefore, depends on several factors: national demographic characteristics, the local epidemiology of the disease, identification of known risk behaviors, and cultural understandings related to risky behavior. The limited prevalence data and lack of general understanding of risk factors of the bridging population in Pakistan contributed to this study's underlying objective of improving understandings of the bridging population. The approach for defining the bridging population in this study is based on what is known about prevalence data and risk behaviors in the context of Pakistan.

This chapter of the report presents: the criteria used to define and identify the bridging population; the study results in terms of the bridging population's sexual risk behaviors within the overall picture of sexual behavior in Pakistan; and bivariate and multivariate analyses of the bridging population's sociodemographic characteristics.

\section{Definition}

To determine the bridging population in Pakistan we adopted the traditional definition of the 'bridging population' visually described in figure 7.1. 
Figure 7.1: Sexual network and STI/HIV transmission

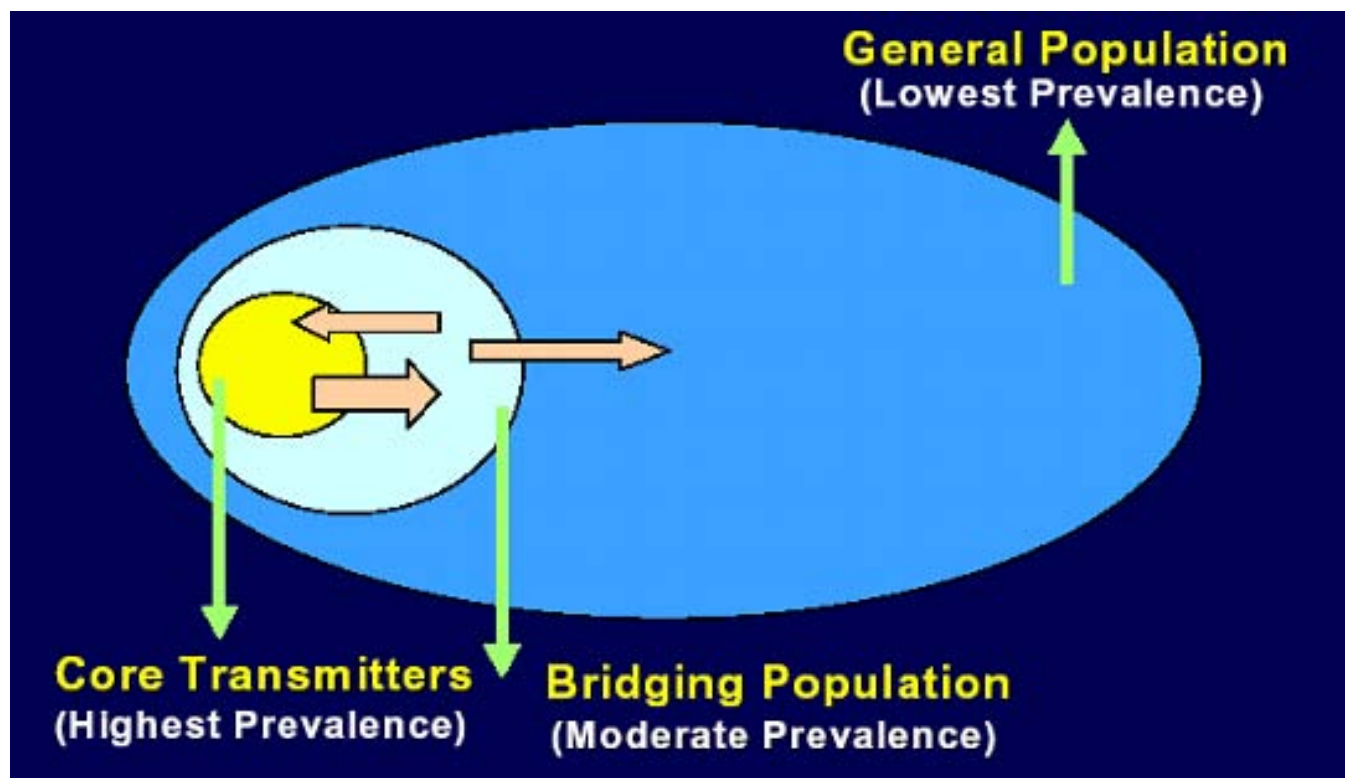

Aral (1999); Van Dam (1999)

The visual presented in figure 7.1 illustrates how STIs and HIV/AIDS move from core or high-risk groups, such as sex workers, through a bridging population into the general population. Based on a review of the literature on prevalence and risk behaviors in South Asia and Pakistan, these core or high-risk groups include:

- Female sex workers (Nanan et al. 2000)

- Male sex workers

- Men who have sex with men (Khan and Hyder 1998; Khan and Khilji 2002; Dandona et al. 2005)

- Hijras (Baqi et al. 1999)

Because this is a study of STIs, we concentrate on sexual behavior as the mode of transmission for the bridging population and exclude contact with other core high-risk groups, such as injecting drug users. We include MSM as a high-risk group because engaging in unprotected anal sex increases the risk of HIV and STI transmission and these are typically not monogamous relationships. The general population comprises sexually active adults who otherwise are considered to be at low risk of acquiring an STI.

We conceptualize the bridging population by taking into account the definitional differences between two categories of men: married and unmarried. For the married population, the bridging population includes those men who report extramarital 
sexual contact with one of the high-risk groups (their contact with the general population is through their wives). For unmarried men, the bridging population includes those men who report premarital sex with more than one type of partner, with at least one partner being a member of the high-risk group (their contact with the general population is through female other than sex workers).

Our definition of the bridging population focuses on sexual behaviors within the last 12 months. This time period was selected for several reasons: 1) it is a common reference period for studies of the bridging population; 2) it is long enough to capture risk behaviors, but not too long to be adversely affected by respondent recall bias; and 3) it allows for some comparisons over time (i.e., with those respondents reporting non-marital sex in the last three months) and for picking up the markers for serological testing.

Table 7.1 presents the conceptualization of our definition of the bridging population. This definition is based on the parameters described in studies from the international literature (Aral 1999; Van Dam 1999; Morris et al. 1996; Lowndes et al. 2002) and the literature on high-risk groups in Pakistan (for example, Khan and Hyder 1998; Khan and Khilji 2002; Baqi et al. 1999).

Table 7.1: Defining the STI bridging population in Pakistan

Core group contact:

High-risk sexual behavior group

1. Married men. The man has had extramarital sex with at least one of the following type of sexual partners in the last 12 months:

- Female sex worker (FSW)

- Male sex worker (MSW)

- Male other than sex worker (MOSW)

- $\quad$ Eunuch (hijra)

2. Unmarried men. In the last 12 months the man has had premarital sex with at least two partners with one of them being one of the following type of sexual partners:

- Female sex worker (FSW)

- Male sex worker (MSW)

- Male other than sex worker (MOSW)

- $\quad$ Eunuch (hijra)
BRIDGE General population contact: Low-risk sexual behavior group

The man is currently married.
AND The man has had sexual activity with female other than sex worker. 
We define the general population to include wives and females other than sex workers. The rationale for this is that men who report premarital or extramarital contact with female friends may reflect a long-term or steady relationship with a female who is not part of the high-risk population.

We recognize that the specific characteristics of the definition could be enhanced somewhat to identify the potential bridging population. However, we feel confident that this reflects a strictly defined bridging population.

\section{Non-marital sexual behavior}

Our study of the bridging population requires identifying the sexual behaviors (i.e., type of partner) of those men who report non-marital sex in the last 12 months.

Fifteen percent of all study respondents reported having had non-marital sex in the last 12 months, while nearly one out of every eight respondents reported non-marital sexual activity in the last three months. Table 7.2 shows the type of non-marital sexual partner over the last 12 months. Non-marital sex was most commonly reported with females other than sex workers: in all six cities, the highest proportion of respondents reported having non-marital sex in the last 12 months with a female friend.

The next most frequently reported category of non-marital partner was female sex workers. This was highest in Karachi (15 percent), followed by Faisalabad (14 percent) and Quetta (13 percent). The lowest proportion of respondents reporting sex with a female sex worker was in Rawalpindi (9 percent). The third highest non-marital sexual liaison was reported with males who were not sex workers; the highest proportion was reported in Faisalabad (10 percent), followed by Rawalpindi (8 percent). Sex with a male sex worker was less than 10 percent in all six cities: the highest proportion was in Karachi at 3 percent, while the lowest was in Lahore, where only one respondent reported such a liaison.

Sex with eunuch or hijra, was less than 5 percent in all six cities. Based on the findings of the qualitative research, we classified hijra as a separate category of male sexual partner. The highest proportion within this category was reported in Faisalabad (3 percent), followed by Karachi ( 2 percent). In Rawalpindi, only three respondents reported having had such a liaison. 
Table 7.2: Type of non-marital sexual partner over last 12 months, by city

\begin{tabular}{lrrrrrrr}
\hline Type of partner & Faisalabad & Karachi & Lahore & Peshawar & Quetta & Rawalpindi & Overall \\
\hline FOSW & 69.4 & 53.2 & 68.9 & 50.0 & 55.2 & 62.5 & $\mathbf{6 0 . 8}$ \\
FSW & 30.6 & 43.0 & 51.1 & 38.2 & 41.4 & 30.4 & $\mathbf{3 8 . 1}$ \\
Male $^{\mathrm{a}}$ & 25.5 & 36.7 & 13.3 & 35.3 & 36.2 & 21.4 & $\mathbf{2 6 . 8}$ \\
$\mathbf{( N )}$ & $\mathbf{( 9 8 )}$ & $\mathbf{( 7 9 )}$ & $\mathbf{( 4 5 )}$ & $\mathbf{( 3 4 )}$ & $\mathbf{( 5 8 )}$ & $\mathbf{( 5 6 )}$ & $\mathbf{( 3 7 0 )}$ \\
\hline
\end{tabular}

a "Male" includes: male friends, male sex workers, and hijras.

Table 7.3 shows the proportions of male sexual partners and paid sex with men during the last 12 months. Five percent of respondents reported having sex with a male (paid, unpaid, or hijra) in the last 12 months. Of these, the highest number reported having sex with a male friend ( 3 percent). It is possible that due to social stigma there was some underreporting of sex with men. Other studies (Khan and Hyder 1998; Khan and Khan 2006) and anecdotal

Table 7.3: Respondents according to type of male sexual partner within the last 12 months

\begin{tabular}{lrr}
\hline Type of partner & Proportion & Percent \\
\hline Total & $\mathbf{1 2 3 / 2 , 4 0 0}$ & $\mathbf{5 . 1}$ \\
MSW (paid) & $33 / 2,400$ & 1.4 \\
MOSW (unpaid) & $72 / 2,400$ & 3.0 \\
Hijra & $18 / 2,400$ & 0.8 \\
\hline
\end{tabular}
evidence suggest that males engaging in sex with other males is a more frequent practice.

Number of non-marital sexual partners. Respondents who reported non-marital sex in the last 12 months $(n=370)$ were asked to report the number of non-marital sexual partners they had in the last 12 months. While the minimum number of partners was one, the maximum number reported by one respondent was 50 . We grouped the reported number of partners into three categories of those who reported one nonmarital sexual partner; two non-marital sexual partners; or three or more non-marital sexual partners. The analysis across cities shows that 48 percent of the respondents had only one sexual partner, 20 percent had two; and 32 percent reported more than three partners in the last 12 months.

Figure 7.2 shows the percentage of numbers of non-marital sexual partners broken down by marital status with $95 \%$ confidence intervals. The results suggest there was no significant difference in number of non-marital partners based on marital status. 
Figure 7.2: Percentage distribution, with $95 \% \mathrm{Cl}$, of respondents according to number of non-marital partners in last 12 months, by marital status

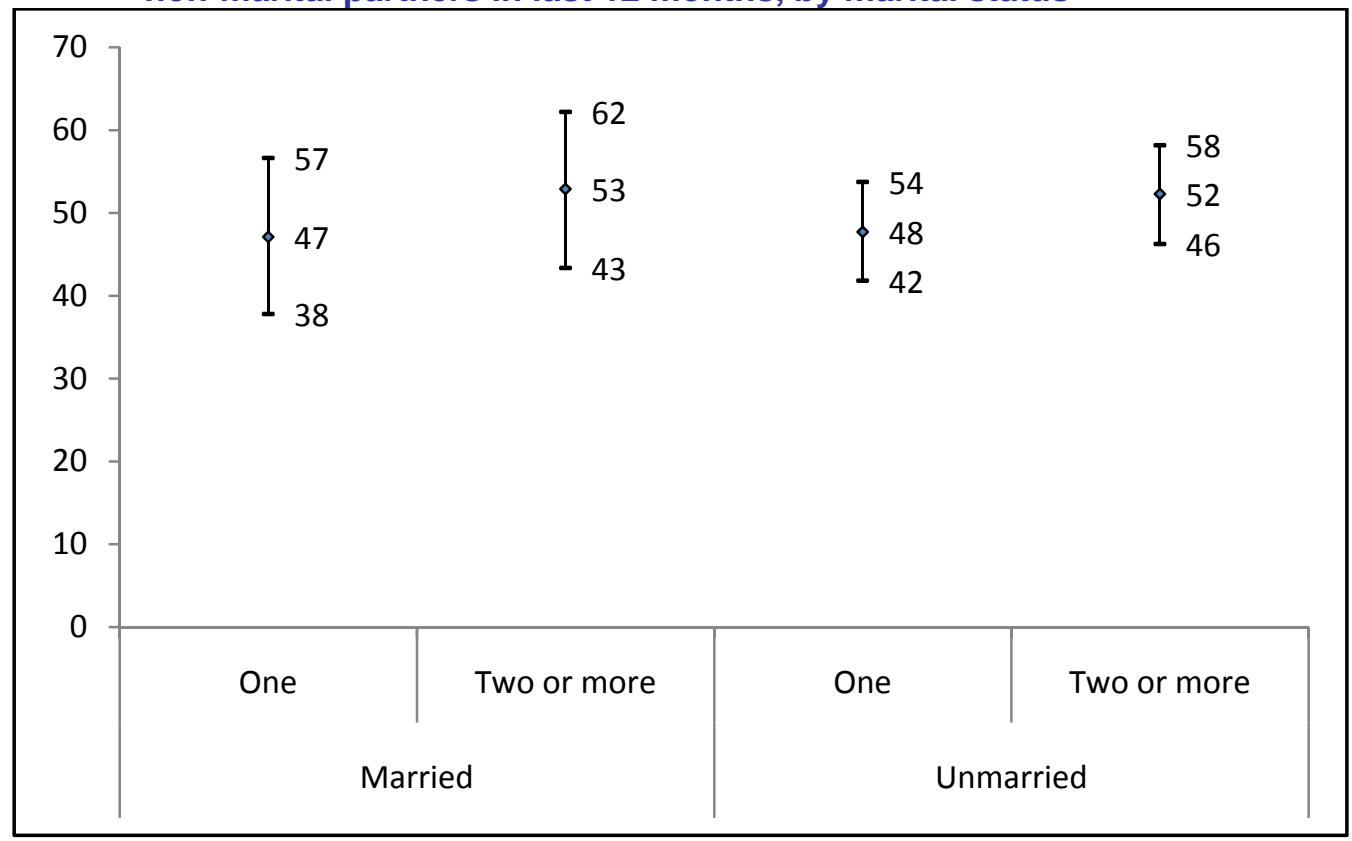

Bivariate analysis was carried out to assess if the age of the respondent was associated with number of sexual partners. There does not seem to be a clear relationship between age and number of non-marital partners, although respondents reporting three or more sexual partners were higher in the age range of 16-30 years.

\section{Sociodemographic characteristics}

Based on these results of non-marital sexual behaviors over the last 12 months and our definition and assumptions about the bridging population, our bridging population estimate becomes 206 men out of the total study population.

The following tables and graphs present the sociodemographic characteristics for the bridging population in our study. These data are presented for both the bridging population $(n=206)$ and the non-bridging population (the remaining study population $n=2,194)$ to allow for identification of differences between the bridging and nonbridging populations. 


\section{Age}

The mean age of the bridging population was 25 years and the median age was 23 years. While there was some variation across the six cities, the age of the bridging population was primarily distributed across the ages 21-25, except in Peshawar, where there was a more even distribution across the age groups of 16-20 years, 21-25 years, and 31-35 years (see appendix 1 for breakdown by city).

Figure 7.3: Age of bridging and non-bridging population

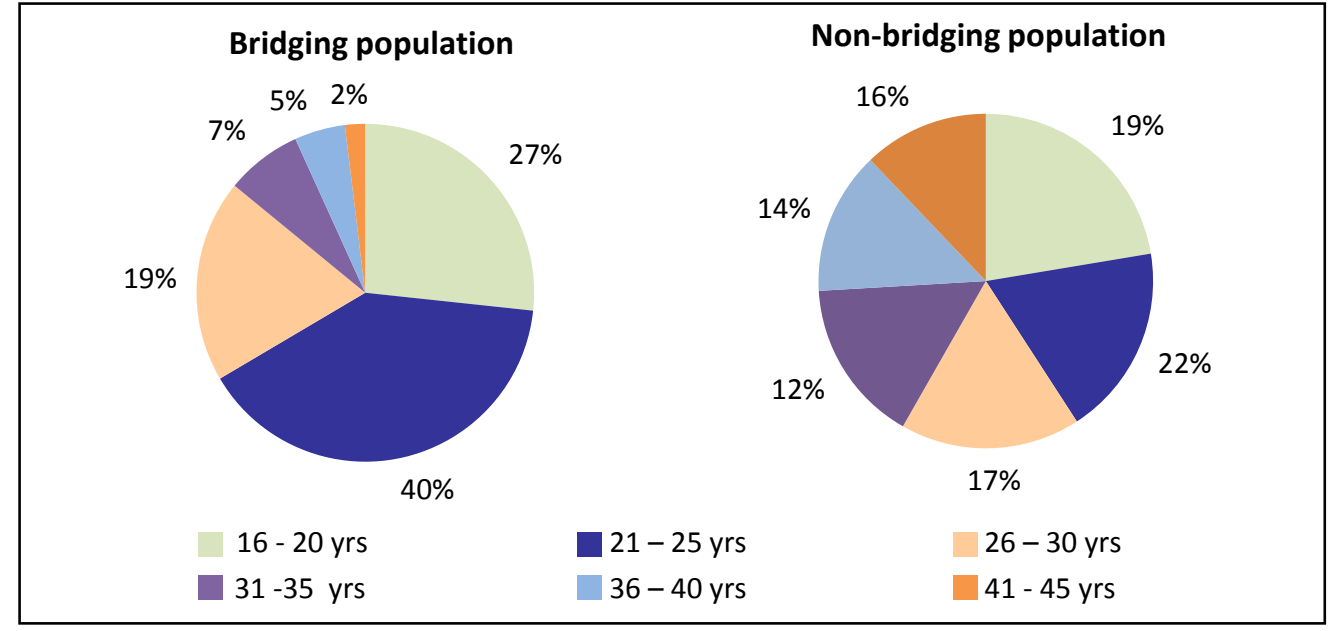

Comparing the $95 \%$ confidence intervals of bridging versus non-bridging populations shows that bridgers were significantly $(\mathrm{p}<.05)$ more likely to be in the 16-20 and 21-25 year age categories. Non-bridgers were significantly more likely to be in the 36-40 and $41-45$ year age groups $(\mathrm{p}<.05)$. (Results are shown in appendix 1.)

\section{Marital status}

The majority of respondents in the bridging population (74 percent, $n=152$ ) were unmarried. There was slight variation between cities, with a greater number of unmarried men in the bridging populations in Lahore and Karachi. Higher numbers of married men comprised the bridging populations in Peshawar and Quetta.

A smaller percentage of the non-bridging population was unmarried than the bridging population (45 versus 74 percent) (figure 7.5). Being unmarried was a significant difference between the bridging and non-bridging populations $(\mathrm{p}<.05)$. (Results are shown in appendix 1.) 
Figure 7.4: Marital status of bridging and non-bridging population

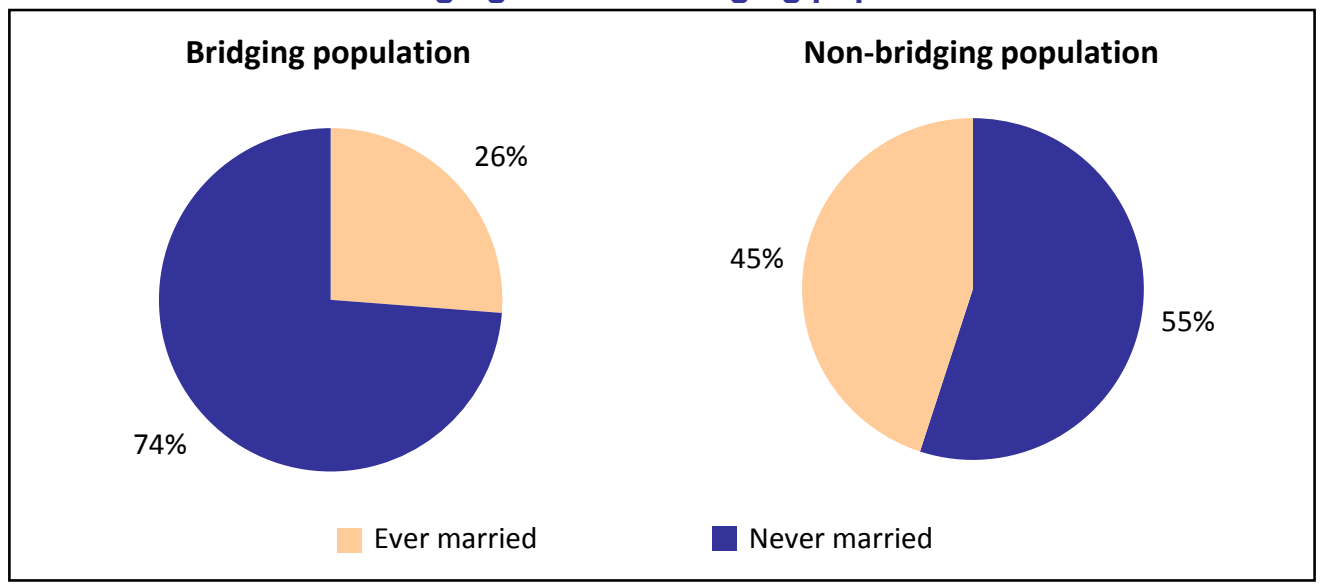

\section{Education}

Across all six cities, the majority of men in the bridging population had some education. In the total sample of the bridging population, 40 percent of men had primary education. Peshawar exhibits especially high educational status of the bridging population, with almost 70 percent of respondents reporting secondary education or above.

A significantly higher proportion of the bridging population had only primary education (40 percent) versus the non-bridging population ( 23 percent), and a higher proportion of the non-bridging population had been educated above the secondary level (33 versus 17 percent) $(\mathrm{p}<.05)$ (figure 7.6$)$. There was no significant difference in the illiterate or secondary educated populations. 
Figure 7.5: Educational status of bridging and non-bridging population

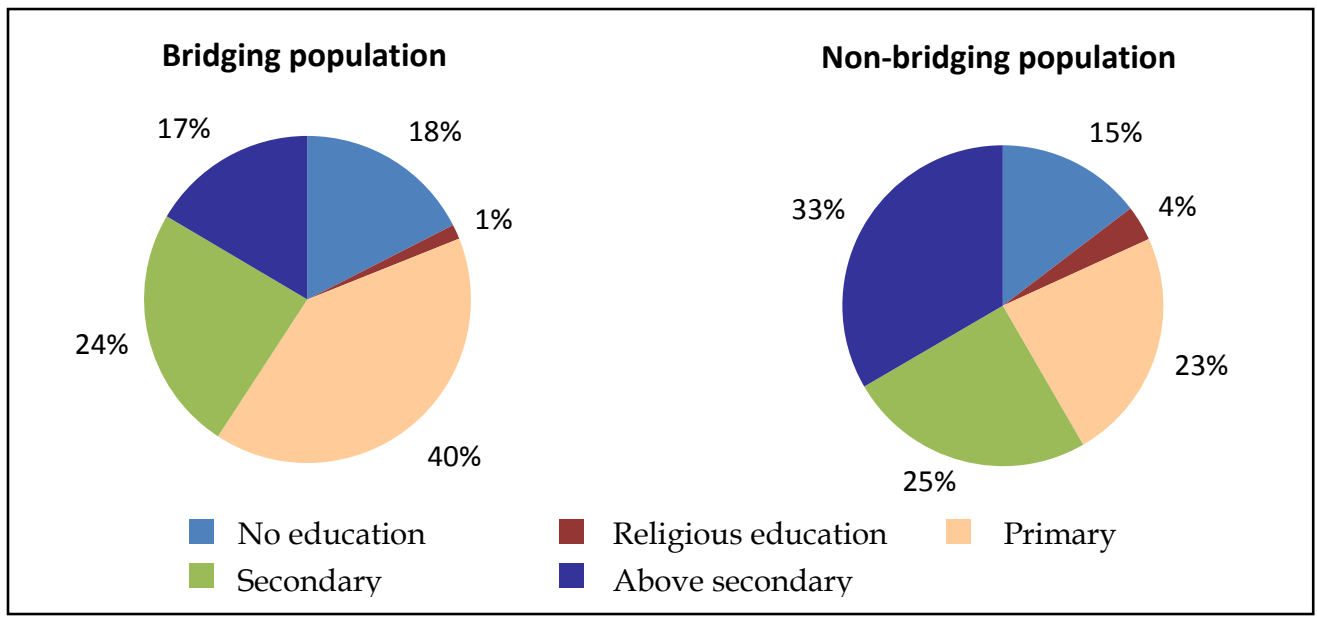

\section{Occupational status}

In terms of self-reported occupational status in the bridging population across the six cities, the majority of respondents reported working in private service (40 percent) or being self-employed (36 percent) (figure 7.6). Those in the bridging population were significantly more likely to be working in private service and significantly less likely to be working in government service than in the non-bridging population (see appendix 1 for comparison between bridging and non-bridging with $95 \%$ confidence intervals).

Figure 7.6: Occupational status of the bridging and non-bridging population

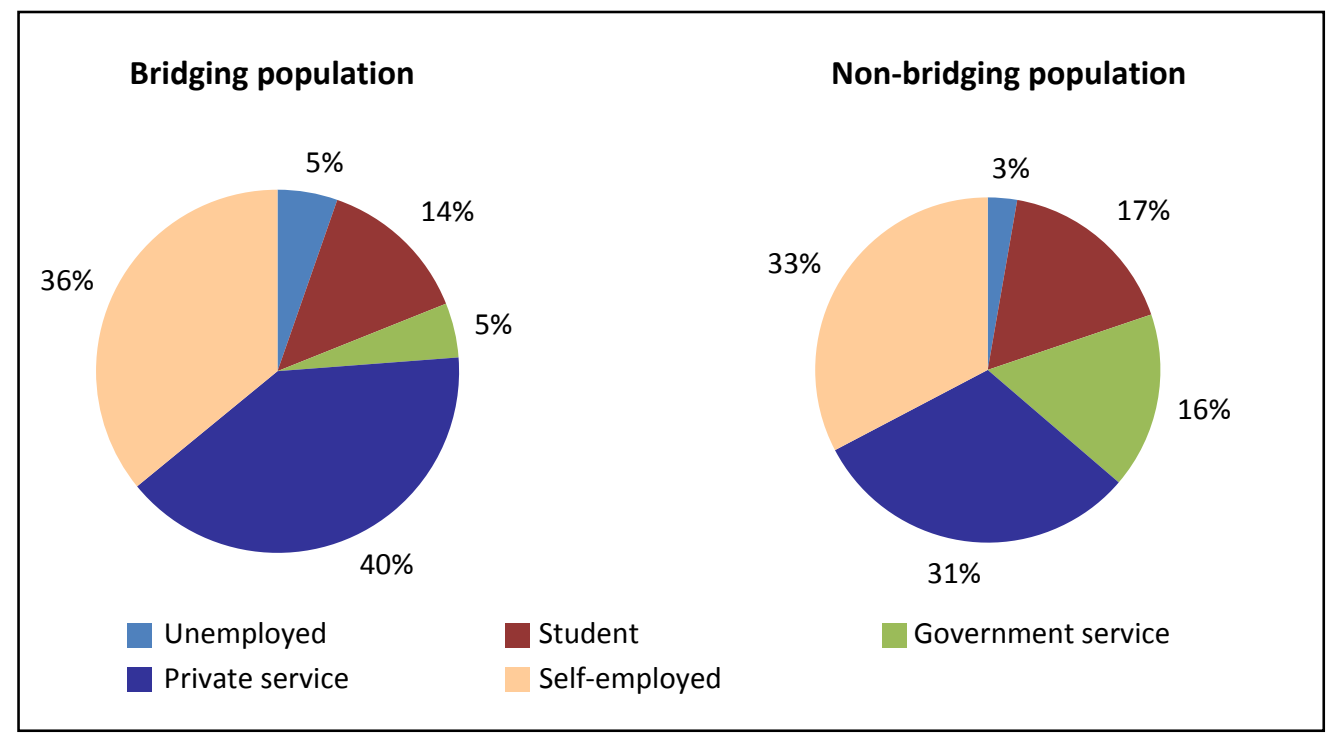




\section{Earnings}

Self-reported earnings (salary) per month were classified into four categories: student/unemployed; up to Rs. 5,000; between Rs. 5,001 and 15,000; and more than Rs. 15,000 .

In the bridging population, 19 percent were students or unemployed (figure 7.7). Forty percent reported earning Rs. 5,000 or less each month, and 35 percent reported earning between Rs. 5,000 and 15,000. Six percent of the bridging population earned more than Rs. 15,000 per month.

The bridging population was more likely to be students or unemployed and earning less than Rs. 5,000 per month than the non-bridging population. The non-bridging population was more likely to be earning Rs. 5,000 or more per month than the bridging population (see appendix 1 for results with $95 \%$ confidence intervals).

Figure 7.7: Self-reported monthly earnings of bridging and non-bridging population, in Pakistani rupees

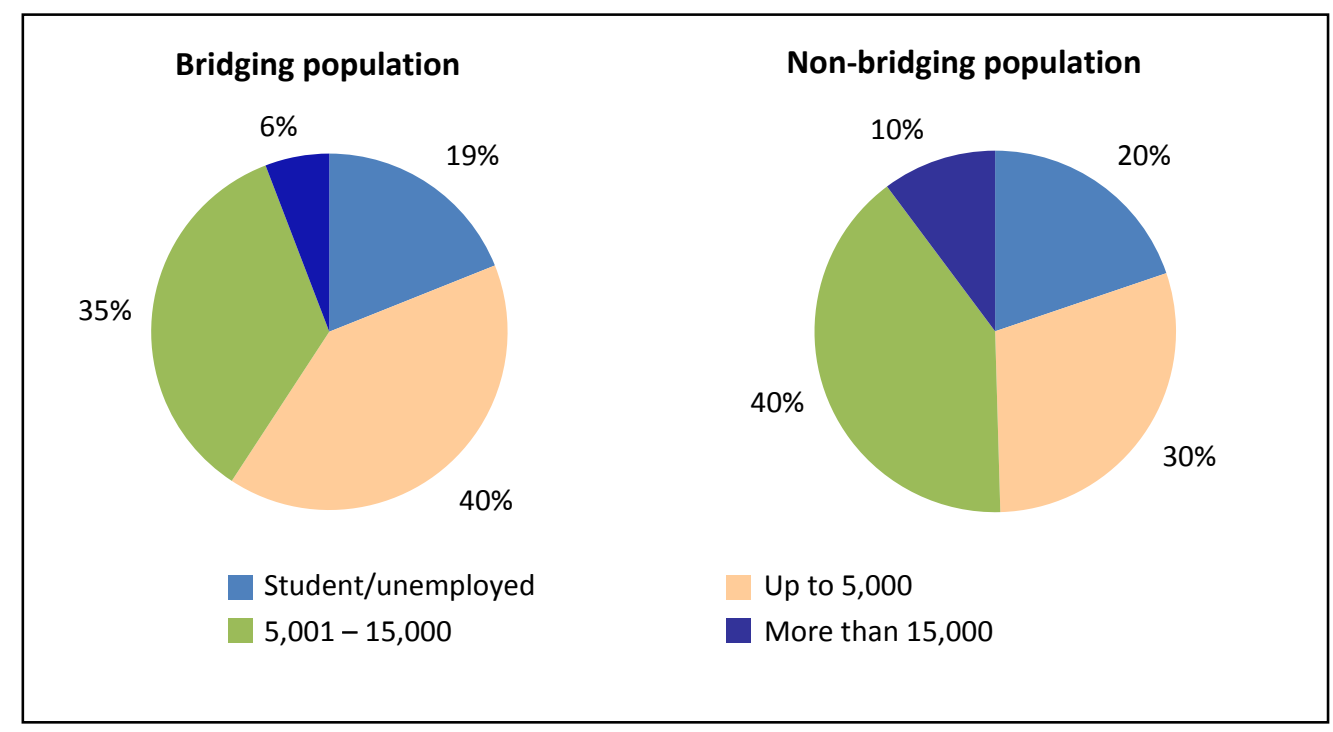

\section{Migrant status}

Migrant status is considered to be a potential risk factor because of the perceived likelihood that men will engage in more risky behaviors in the absence of the behavioral constraints of their social setting. As a result, the migrant population has been defined as the bridging population in other studies (Faisel and Cleland 2006; Lau 
et al. 2000). We examined the bridging population by migrant status (defined as living away from marital or natal home for the last four months). In the bridging population, 18 percent were migrants and 82 percent were non-migrants (figure 7.8). This compares with 8 percent migrant population in the non-bridging group. So more migrants were found among the bridgers as compared to non-bridgers and this difference was statistically significant.

Figure 7.8: Migrant status of bridging and non-bridging

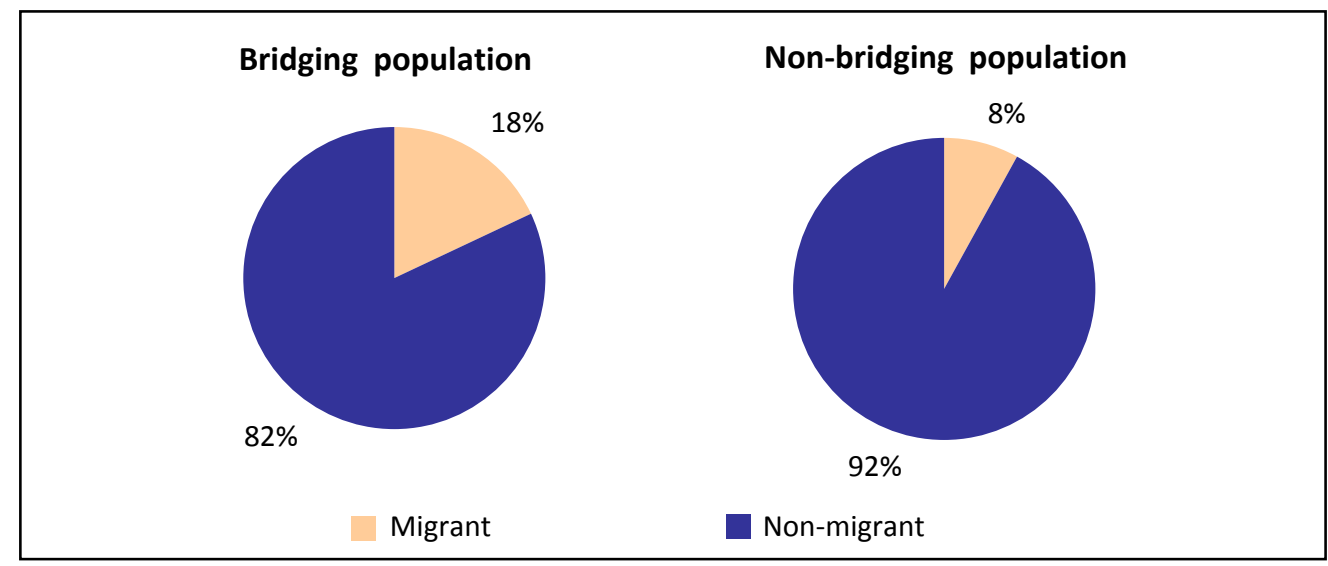

\section{Mobility}

Similar arguments as to why migration status (living away from home) is a potential risk factor can also be applied to men who spend nights outside their home and away from their family. These are men who are mobile or 'men on the move' based on their occupational requirements. In our study, we refer to these men as 'travelers'. If a respondent reported spending one or more nights away from home in one month as part of his job, he was classified as a traveler. Sixteen percent of the bridging population met this definition of a traveler; 84 percent did not travel outside their homes for business purposes (figure 7.9). This compares to 16 percent in the general study population suggesting no difference between the bridging and the non-bridging population in terms of traveler status. 
Figure 7.9: Traveler status of bridging and non-bridging population

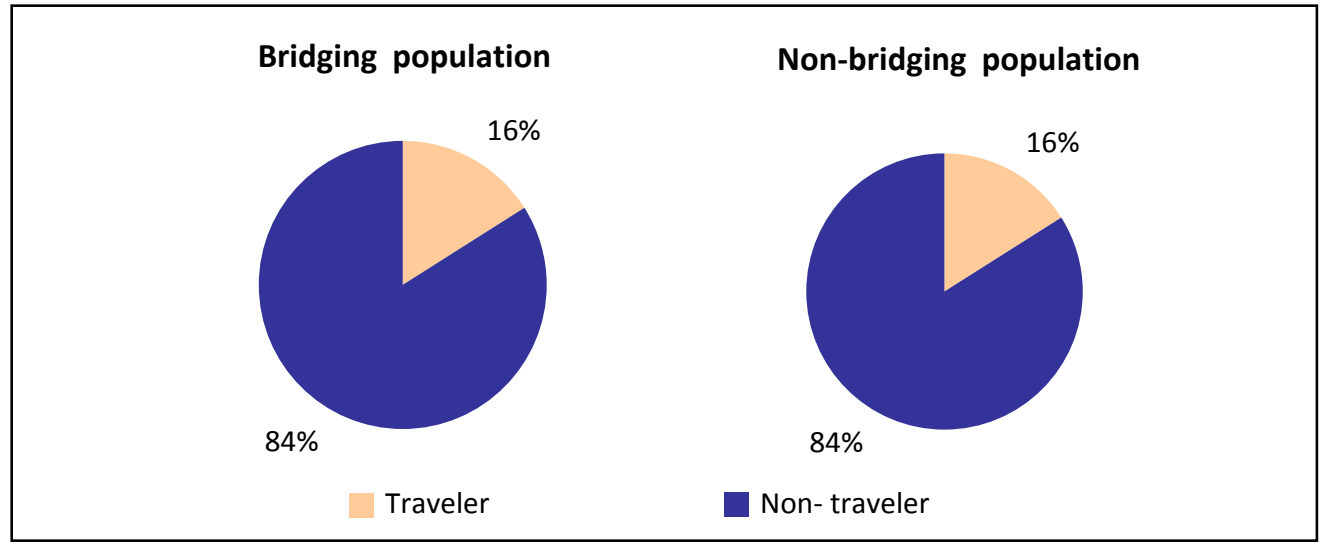

\section{Sexual behaviors and STI prevalence}

We examined the STI Table 7.4: STI prevalence within the bridging and nonprevalence in the bridging population ( 7 percent) and compared it with the STI prevalence in the non-bridging population bridging populations

\begin{tabular}{lcrr} 
& STI positive & STI negative & \multicolumn{1}{c}{ Total } \\
\cline { 2 - 4 } Population & $\%$ & $\%$ & $\mathrm{~N}$ \\
\hline Bridging & 6.8 & 93.2 & 206 \\
Non-bridging & 4.2 & 95.8 & 2,177 \\
\hline
\end{tabular}
(4 percent). We found no statistically significant difference table 7.4 between the two.

However, when we compared STI prevalence among those respondents who reported lifetime non-marital sex with high-risk groups and including 'friends' with those who never had non-marital sex (virgins and monogamous married men), the difference in STI prevalence between the two groups becomes significant. The results in table 7.5 show that when the category 'female friend' as a non-marital sexual partner is included in both lifetime and last 12 months, the relationship between STI prevalence and non-marital sexual behavior becomes significant ( $p$-value <.01). 
Table 7.5: STI prevalence with reference to lifetime and last 12 month non-marital sexual behaviors

\begin{tabular}{lccr}
\hline & & \multicolumn{2}{c}{ STI prevalence } \\
\cline { 2 - 4 } Time period and sexual behavior & STI positive & STI negative & Total \\
\cline { 2 - 4 } Lifetime & Percent & Percent & \\
Had non-marital sex & & & 691 \\
Did not have non-marital sex & 3.4 & 96.6 & 1,692 \\
\hline Within last 12 months & 3.2 & & \\
Had non-marital sex & & 91.9 & 370 \\
Did not have non-marital sex & 8.1 & 96.3 & 2,013 \\
\hline *For both lifetime and last 12 months, STI prevalence is significantly higher among those who reported \\
non-marital sex as compared to those who did not report non-marital sex. The p-value for both statistics \\
was <.01.
\end{tabular}

Eighteen percent of the bridging population reported experiencing one or more of three STI symptoms, as shown in table 7.6. This compared with 6 percent of the non-bridging population. This difference in reporting STI symptoms was found to Table 7.6: Percentage of bridging and nonbridging population self-reporting STI symptoms

\begin{tabular}{lrrr}
\hline & \multicolumn{3}{c}{ STI symptoms } \\
\cline { 2 - 4 } Population & Yes & No & N \\
\hline Bridging & 18.4 & 81.6 & 206 \\
Non-bridging & 5.7 & 94.3 & 2,194 \\
Total & $\mathbf{6 . 8}$ & $\mathbf{9 3 . 2}$ & $\mathbf{2 , 4 0 0}$ \\
\hline
\end{tabular}
be statistically significant.

Sixteen percent of men in the bridging population reported using a condom at last non-marital intercourse (table 7.7). This compares to 4 percent of men in the nonbridging population who reported using a condom at last non-marital sexual intercourse. This is an interesting finding that requires more explanation. One reason for the higher rates of condom use in the bridging population may be that they were a younger, more sexually active population with greater exposure to pornography. These men could be less inhibited and more comfortable acquiring and using condoms.

Table 7.7: Percentage of bridging population and non-bridging population reporting condom use during last non-marital intercourse

\begin{tabular}{lrrr}
\hline Population & Used condom last time & Did not use condom last time & N \\
\hline Bridging & 16.0 & 84.0 & 206 \\
Non-bridging & 3.6 & 96.4 & 2,194 \\
Total & $\mathbf{4 . 7}$ & $\mathbf{9 5 . 3}$ & $\mathbf{2 , 4 0 0}$ \\
\hline
\end{tabular}




\section{Multivariate analysis}

Logistic regression analysis was used to explore the relationships between the variables and to adjust against possible confounding effects of other characteristics. The final model was selected based on significant sociodemographic factors associated with the bridging population; tables 7.8 and 7.9 show crude and adjusted odds ratios for the final model.

The unadjusted results suggest that having ten years or less of education was a factor associated with being part of the bridging population (2.5 unadjusted OR). Being below the age of 27 years and being unmarried also appear to be factors associated with the bridging population. Living with extended family was also a significant factor (1.9 unadjusted OR).

Table 7.8: Sociodemographic factors associated (unadjusted) with bridging and nonbridging population

\begin{tabular}{lrrr}
\hline & \multicolumn{2}{c}{ Population } & Unadjusted \\
\cline { 2 - 3 } Variable & Bridging & Non-bridging & OR (95\% Cl) \\
\hline Education & & & \\
Up to 10 years & 171 & 1,455 & $2.5(1.8-3.7)$ \\
$>10$ years & 34 & 733 & 1 \\
\hline Age & & & \\
$<27$ years & 155 & 1,055 & $3.3(2.4-4.6)$ \\
$>27$ years & 51 & 1,139 & 1 \\
\hline Living arrangement & & & \\
Extended family & 32 & 191 & $1.9(1.3-2.9)$ \\
Nuclear/joint family & 174 & 1998 & 1 \\
\hline
\end{tabular}

Logistic regression analysis was used to explore the relationships between the variables and to adjust against possible confounding effects of other characteristics. The multivariate model controlled for the variable 'type of partner' and marital status as they are part of the definition of the bridging population. The final model was selected based on significant socio-demographic factors associated with the bridging population. The adjusted odds ratios for the final model are shown in table 7.9. 
The results of the model suggest that being below the age of 27 years, having some education (up to 10 years), and living with extended family as opposed to nuclear (parents or wife) or joint (with parents and wife) family are sociodemographic factors associated with being included in the bridging population. We tried including migrant status and traveler status as variable in the model but they were not
Table 7.9: Sociodemographic factors associated (adjusted) with bridging population

Adjusted OR (95\%

\begin{tabular}{lr} 
Variable & $\mathrm{Cl})$ \\
\hline Education & \\
Up to 10 years & $1.9(1.2-3.0)$ \\
$>10$ years & 1.0 \\
\hline Age & $1.7(1.3-3.8)$ \\
$<27$ years & 1.0 \\
$>27$ years & \\
\hline Living arrangement & $2.2(1.3-3.8)$ \\
Extended family & 1 \\
Nuclear/joint family &
\end{tabular}

significant. The effect of living arrangement may be due to increased freedom of behavior among those living with in a larger family unit. It may also reflect increased access to potential partners.

\section{Chapter summary}

This chapter has described the basic sociodemographic characteristics of the population of men who may be the most likely to transmit STIs from high risk groups to the general population. These men are younger than the general study population (mean age 25 versus 29 years; median age 23 versus 27 years), and they are less likely to be married (74 percent are unmarried). The majority have up to secondary education; and are working in the private sector (40 percent) or are self-employed (36 percent). Eighteen percent are migrants. The low number of migrant men in the bridging population reinforces the decision to design the study so as to reach the general population in our study of urban men. Even when including travelers (16 percent of the bridging population sample) the majority of the bridging population would not have been included.

After adjusting for possible confounding effects, the sociodemographic characteristics associated with being in the bridging population become clearer: being below the age of 27 years (1.7 adjusted OR); having ten years or less education (1.9 adjusted OR); and living in an extended family situation ( 2.2 adjusted OR).

Comparing STI prevalence between the bridging and the non-bridging population did not yield a statistically significant difference. However, when the category of female other than sex worker or 'female friend' was included there was a significant difference between STI prevalence, both over lifetime and in the last 12 months (p- 
value<.01). This finding suggests that the female other than sex worker or 'female friend' is an important factor in STI prevalence and better understanding of who these females are is required. The qualitative research component of the study helps to shed some light on some of the general behaviors surrounding unpaid non-marital sex (see appendix 3). 


\section{DISCUSSION AND RECOMMENDATIONS}

\section{Discussion}

This study is unique in terms of its subject matter (urban male sexual behaviors and attitudes), study population (urban men from the general population), and methodology (biological testing for STIs in conjunction with a detailed behavioral questionnaire and in-depth qualitative research). The findings of this study provide evidence about a subject area for which there has been little data in the past and the results challenge preconceived notions about men's sexual behaviors and attitudes in Pakistan.

The findings show that premarital and extramarital is taking place among urban men: 29 percent of men reported non-marital sex, with 13 percent engaging in extramarital sex and 16 percent in premarital sex. Twenty-two percent of those who had nonmarital sex reported having three or more non-marital partners in the last twelve months. These findings about men's sexual behavior may be somewhat surprising given the traditional sociocultural context of Pakistan; however, these results are not unlike other studies of male sexual behavior in South Asia (Collumbien 2000).

Within this context of non-marital sex, there is, indeed, high awareness of HIV/AIDs. However, there is poor understanding of the modes of HIV transmission and the methods to prevent HIV transmission. There is also very limited knowledge of STIs other than HIV/ AIDS and it is these other STIs that had the highest prevalence in our study. Within this context of limited knowledge about STI transmission, the study found very low rates of condom use. Only 16 percent of men reported using a condom during their last non-marital sexual intercourse, and only 9 percent of those reporting STI symptoms used a condom at last non-marital sex. The study is the first of its kind to identify the bridging population in Pakistan. The results suggest that the bridging population is more expansive than migrant men or 'men on the move.' Had this study not expanded the definition of the bridging population to include urban men, the understanding of the specific characteristics of this population would have been lost. 
Successfully identifying the characteristics of the bridging population and understanding their risk behaviors is critical for designing interventions that will diminish the spread of HIV and other STIs in Pakistan.

\section{STI prevalence}

The study's biological findings of a prevalence rate of 4.4 percent for five common STIs, including HIV/AIDS, are within the predicted limits. However the study also points out that there are city wise variations in STI prevalence, with Karachi having the highest prevalence of nearly 8.5 percent. The highest prevalence of HSV2 infection was also seen in Karachi at 6 percent. They are also within the range of other studies in South Asia (Hawkes et al. 1999; Faisel and Cleland 2006). Some general explanations that might explain the STI prevalence results include the almost universal practice of male circumcision in Pakistan. In this study, 97 percent of respondents reported being circumcised. Male circumcision has been documented to be a protective factor in the spread of HIV (Williams et al. 2006). There is now compelling epidemiological evidence from over 40 studies that shows that male circumcision provides significant protection against HIV infection: circumcised males are two to eight times less likely to become infected from HIV (Halperin and Bailey 1999). Second, maintaining genital hygiene around sexual intercourse, thought to be protective against STI transmission (O'Farrell 1993), is commonly practiced and is religiously sanctioned. Furthermore the study used infection rather than exposure as a marker for STI disease. Infection measures acute STI infection rather than chronic STI infection. Had we used exposure to STIs as the marker, we might have had a higher STI prevalence rate.

Importantly, the behavioral and qualitative components of the study identify sexual risk behaviors that may have important implications for the future trajectory of the epidemic. The first are the rates of premarital and extramarital sex reported in the study. Twenty-nine percent of men reported that they had engaged in either premarital or extramarital sex in their lifetime. While there are no similar studies among the general population of men in Pakistan with which to compare these results, this finding is in the range of other studies of sexual behavior in South Asia (Collumbien 2000).

The study findings also contradict the often widely held preconceived notions that religious and sociocultural norms are sufficient for containing the HIV epidemic in Islamic countries such as Pakistan (Obermeyer 2006; Kelley and Eberstadt 2005; Gray 2004). This study shows that non-marital sexual activity is taking place, and that those 
males who engaged in extramarital sex (in all but 2 percent) also had premarital sexual experience. This suggests that those who engaged in premarital sex were more likely to continue non-marital sexual behaviors after marriage. The reasons for this remain unclear; however, it does suggest that interventions should target unmarried men as their sexual risk behaviors begin before they are married. Marriage in Pakistan is almost universal, implying that those men engaging in premarital sexual behaviors may likely continue their non-marital sexual behaviors once they are married. Men who have had premarital sexual experience may enter their marriage already infected or have been exposed to an STI thus placing their wives at risk for infection.

The second concern raised by the study has to do with the sexual behaviors and attitudes of young males. Sexual activity in Pakistan begins at young ages, and with the increasing age of marriage, the window of sexual opportunity is widening, allowing greater opportunity for engaging in risky sexual behaviors. The data from this study suggest that sexual experimentation is common among young males (between the ages of 16-20) both in terms of the frequency of sex and also by the type of sexual partner. The study data show that sexual experimentation at younger ages is more likely to occur with other males (friends) or a sexual partner who is easily available (female friend or relative). The selection of sexual partners among this age group may be explained by the limited financial resources and independence available at younger ages. The study results show that as men get older, perhaps because they have greater financial resources at their disposal, they are more likely to access sex workers.

The mean age of sexual debut in the study population was 22.3 years. When the mean ages for sexual debut are broken down by marital status, the mean age of sexual debut for married respondents was 23.4 years and for unmarried it was 18.6 years. While subject to cautious interpretation, this suggests that interventions should target young unmarried men who are more likely to engage in sexual risk behaviors. With a large cohort of Pakistan's population currently in the adolescent age groups, it is important that school health education programs be initiated that equip youth to lead healthy lives by overcoming peer pressures and making sound personal decisions.

The qualitative study findings also revealed that men use a combination of sexual partners. Men felt that sex was a natural desire, which they could not control and, if necessary, many were willing to have sex with whoever was available. Some men indulged only with sex workers and the qualitative data in this study report that 
transsexuals and eunuchs were rarely used for sexual purposes. However, overall the qualitative data show that unpaid sex is common. Men usually preferred females for sex, but younger unmarried men also developed liaisons with men as part of sexual experimentation and for reasons of affordability and availability.

\section{Condom use}

The findings on condom use raise concerns for the future of STI transmission in Pakistan. Among all respondents, condom use at last non-marital sex was 16 percent and only 10 percent of those men who had non-marital sex in the last 12 months reported consistent condom use. Furthermore, men rarely used condoms when having sex with other men. In the case of sex with males, the main reason for not using a condom was that respondents felt it was not necessary, suggesting that respondents perceive condoms to be protection against pregnancy rather than as prophylactic against disease. This is further supported by the reports of condom use with female sex workers, which was lower than condom use with females other than sex workers. This might also reflect the level to which the man's partner is empowered to negotiate condom use. For example, men reported extremely low rates of condom use with hijra partners who are generally considered to be very disempowered.

Of all the study respondents reporting STI symptoms, more than 90 percent had not used a condom at last sexual intercourse. In all cities except Lahore (where it was 20 percent), less than 10 percent of respondents reporting STI symptoms had used a condom during last sexual intercourse. These low rates of condom use reflect either a lack of knowledge about STI transmission, and/or unawareness about the protective effects of condoms. In Pakistan, condom use has not been promoted in the HIV IEC campaign. Lack of knowledge coupled with low condom use emphasizes the need for more innovative health education strategies and the need for health education messages that include the promotion of the dual protective role of condom use.

The qualitative study findings corroborate this as well. The IDIs found that although men generally felt vulnerable to STIs because of their non-marital sexual activities, very few had actually used a condom during their last sexual encounter. Awareness about condoms was quite widespread, but many men perceived it as a means to prevent pregnancy rather than protection from STIs. 


\section{Unpaid non-marital sex}

The study reports that a high proportion of non-marital sexual activity is taking place with 'female friends.' As females other than sex workers were excluded from our definition of the bridging population, we excluded them from the analysis of the bridging population. When we examined STI prevalence between the bridging and non-bridging population the results were not statistically significant. However, when we included the category 'friend' as part of the bridging population in the analysis, the difference between STI prevalence between the bridging and non-bridging population became statistically significant. This is evidence that these 'friends' may increase the risk for having an STI infection. In future studies, this group should be considered in the definition of the bridging population.

The importance of this 'friend' sexual partner was also identified by the qualitative study component and this concept requires more elaboration. The qualitative data points out that these 'friends' are often identified through mobile telephones. Such relationships are often described as monogamous; however, it remains ambiguous if these 'friends' in turn have other partners as well. Improving understanding of paid versus unpaid sex also becomes important as these female friends may receive payment in-kind rather than money.

\section{Knowledge and awareness of STls}

An important risk factor for STI transmission has to do with levels of knowledge and awareness regarding sexually transmitted infections including HIV/ AIDS. Our study shows that while most men have heard of HIV/AIDS and some of the modes of its transmission, this knowledge has not been adequately internalized, as men still do not consider themselves to be at risk even while engaging in risky behavior. The study also highlights the existence of several myths and misconceptions about the transmission of HIV/AIDS. One-third of all respondents agreed after prompting that HIV infection can be transmitted through a mosquito bite. These data reflect that a sizeable number of respondents have misconceptions about the spread of infection, which need to be clarified through appropriate and targeted IEC messages. Misconceptions must also be addressed as they can contribute to stigma and discrimination against those with HIV.

The study found that the level of knowledge about STIs, other than HIV/AIDS, and how they are spread remains low among urban men in Pakistan. This is not necessarily surprising given that the present information, education, and communication (IEC) 
messages do not provide specific information on STIs other than HIV/AIDS. The results highlight the importance of expanding the content of these IEC campaigns to include information about all common STIs.

\section{Media and pornography}

The qualitative data suggest that young men's sexual behaviors are influenced by peer pressure and exposure to the media and pornography. Some of the risky sexual behaviors the youth currently engage in are reportedly learned through exposure to pornography in videos/CDs and/or over the Internet. In this context, the behavioral data results indicating that young men are less knowledgeable about STIs and HIV/ AIDS than older men is of added concern.

The exposure to various types of media, including the Internet, may be acting as a catalyst in shaping the attitudes and sexual behaviors of youth. If so, this needs to be countered by information that is equally direct and credible. Socially acceptable messages about the protective factors of abstinence, fidelity, and consistent condom use must be advocated more strongly and effectively.

\section{Care-seeking behaviors}

A majority of the qualitative research participants said that a sound well-reputed doctor should be consulted for the treatment of STIs. The reason given by many was that hakims and homeopaths were not familiar with such problems and further complicated the infection. However, the qualitative data suggest that many STI patients feel more comfortable visiting traditional practitioners for their care seeking. It was reported that, in contrast to doctors, traditional practitioners rarely inquired about personal details; as a result, the visit was perceived to be more confidential. This may have implications for whether or not effective partner management can be addressed. Furthermore, traditional practitioners often misdiagnose diseases which could lead to further complications. This needs to be rectified by improving access to high quality STI care through all levels of the health care system, including basic health units and rural health centers. Furthermore, lady health workers must also be trained to provide appropriate advice and counseling about STIs to women, particularly as they can play an instrumental role in helping with partner management of men who are diagnosed with an STI at the static facilities. 


\section{Self-reporting of STI symptoms}

Our study results suggest that self-reporting of STI symptoms may be a poor predictor of STI infection. Nearly 91 percent of the respondents testing positive based on laboratory diagnosis were asymptomatic. The specificity of three STI symptoms (genital ulcers/sores, pain during urination, and purulent discharge) was low (8.5 percent) while the sensitivity was reasonably high (93 percent). This raises concerns that self-reported STI symptoms will underreport the actual number of STI infections. If this is the case, men will remain unaware they have an STI and may continue to engage in sexual behaviors that increase the transmission of STIs to the general population.

However, we recognize that our results for sensitivity and specificity may be adversely affected because of the six-month reporting period. Some respondents who reported experiencing STI symptoms in the past six months may have had an STI infection that was treated. As a result, these men would test negative for STIs based on laboratory diagnosis. While syndromic management approaches may be appropriate to continue it should be recognized that self-reporting can be unreliable.

\section{Non-sexual behaviors and practices}

The study shows that the use of injections by medical professionals is still very common in Pakistan. The unwarranted use of injections for common medical conditions is often upon the insistence of the patients. This needs to be countered through advocacy messages that target both the providers and the general public. Once the clients stop demanding injections, the practice will wane.

A positive finding of the study has been the almost universal awareness regarding the importance of safe blood and the need for prior testing of blood before it is transfused. There were also relatively high rates of men who reported washing after sex. These genital hygiene practices, in conjunction with almost universal male circumcision in Pakistan, may be important protective factors in limiting the transmission of STIs.

\section{Bridging population}

An important objective of this cross-sectional study was the identification of the characteristics of the bridging population in Pakistan. The study has been able to define, with some confidence, the attributes and characteristics of this population. 
These findings can be used to better identify the target population for IEC and other interventions to prevent transmission of HIV/ AIDS and other STIs.

Our study suggests that the bridging population is essentially young men (under the age of 27) with lower levels of education (less than ten years) and who live with extended family.

\section{Recommendations}

The results of this study reinforce the need to expand and strengthen existing preventive interventions introduced by the Government of Pakistan, as well as to introduce new and cost-effective measures to reduce the spread of STIs to the general population. The recommendations that emerge from this study suggest a threepronged holistic approach that addresses behavior change through IEC strategies, provision of health services (health care delivery), and monitoring of progress (monitoring and evaluation).

\section{Behavior change communication program}

An awareness-raising and sensitization strategy on protection against STIs, which adopts a targeted approach that reaches different segments of society, especially the youth, needs to be introduced. Precise information of all factors that determines the risk of STI/HIV transmission needs to be elaborated, while also mitigating the stigma attached to it. This can be done by utilizing several approaches, such as introducing life skills education programs in schools and health education campaigns in colleges, universities, and in the workplace. Behavior change can only take place if credible information is made available to the people and is periodically reinforced. The focus of all IEC campaigns needs to be directed at reducing risky behaviors, such as number of sexual partners and use of condoms, while also emphasizing the importance of monogamous relationships and delaying sexual debut.

\section{Health care delivery}

Access to integrated reproductive health services needs to be enhanced by ensuring the availability of comprehensive and integrated reproductive health services within all tiers of the health system. It requires a number of initiatives such as change in the medical curriculum to emphasize a holistic approach in identifying and meeting the reproductive health needs of all age groups. Improving the quality of existing STI care being offered within both the public and private sector facilities, through in-service 
refresher trainings that focus on counseling, diagnosis, and management of STIs, as well as the importance of maintaining privacy and confidentiality. With additional trainings, most of the existing health facilities can manage STI patients and also provide youth friendly services. In addition, there is also a need for increasing the number of voluntary counseling and testing services within large urban communities and informing the public about their location and the services that are offered.

\section{Monitoring and evaluation}

Ensuring that reliable information related to STIs is provided in a timely manner is an important step in reducing the likelihood that an STI epidemic will reach the general population. Putting in place mechanisms that can provide reliable and up-to-date information on sexual behaviors and STI prevalence, such as institutionalizing the second-generation surveillance system and expanding it to reach other cities of the country, is necessary. Establishing sentinel surveillance mechanisms within the major urban centers can also help to inform the public health system of the incidence of STIs in a timely manner, enabling a prompt response. In addition, surveys on the bridging population need to be replicated periodically to gauge trends over time. Using selected indicators, such as the use of condoms and the extent of concurrent partnerships, these surveys can measure the impact of specific programmatic interventions. 



\section{REFERENCES/BIBLIOGRAPHY}

Adrian, M. 2006. “Addiction and sexually transmitted disease (STD), Human Immunodeficiency Virus (HIV), and Acquired Immune Deficiency Syndromes (AIDS): Their mutual interactions." Substance Use and Misuse. 41,12: 1337-1348.

Afsar, HA, MA Mahmood, N Barney et al. 2002. “Community knowledge, attitude and practices regarding sexually transmitted infections in a rural district of Pakistan." Journal of the Pakistan Medical Association. 52: 21-24.

Aggarwal, O. AK Sharma, P. Chhabra. 2000. "Study in sexuality of medical college students in India." Journal of Adolescent Health. 26:226-229.

Agha, A., S. Parviz, M. Younus, and Z Fatmi. 2003. "Socio-economic and demographic factors associated with injecting drug use among drug users in Karachi, Pakistan." Journal of the Pakistan Medical Association. 52: 511-16.

Agha, S. 2002. "Sexual behavior among truck drivers in Pakistan." Culture, Health and Sexuality. 4: 191-206.

Agha, S. 2000. "Potential for HIV transmission among truck drivers in Pakistan." AIDS. 14,15: 2404-2406.

Ahmed, MA, T. Zafar, HJ. Brahmbhatt, et al. 2003. "HIV/AIDS Risk behaviors and correlates of injection drug use among drug users in Pakistan." Journal of Urban Health: Bulletin of the New York Academy of Medicine. 80,2: 321-329.

Akhtar, S., SP Luby, MH Rahbar. 2000. "Multivariate analysis of risk factors associated with genital ulcer disease among incarcerated males in Sindh." Journal of the Pakistan Medical Association. 50: 115-20.

Alam, N. M. Rahman, K. Gausia, et al. 2006. “Sexually transmitted infections and risk factors among truck stand workers in Dhaka, Bangladesh." Sexually Transmitted Infections. Epublication.

Alary, M. and CM Lowndes. 2004. "The central role of clients of female sex workers in the dynamics of heterosexual HIV transmission in sub-Saharan Africa." AIDS. 18,6: 945-947.

Alary, M, CM Lowndes, et al. 2003. "Sexually transmitted infections in male clients of female sex workers in Benin: risk factors and reassessment of the leucocyte esterase dipstick for screening of urethral infections." Sexually Transmitted Infections. 79: 388-392. 
Alexander, M., L. Garda, S. Kanade, S. Jejeebhoy and B. Ganatra. 2006. “Romance and sex: Pre-marital partnership formation among young women and men, Pune District, India." Reproductive Health Matters. 14, 28: 1-12.

Ali, S., R. Kahnani, W. Tariq, SA Shah. 1995. "Understanding the HIV/ AIDS context in Pakistan." Venereology. 8,3: 160-3.

Ali, M., M.A Bhatti, and H. Ushijima. 2004. "Reproductive health needs of adolescent males in rural Pakistan: An exploratory study." Tohoku Journal of Experimental Medicine. 204: 17-25.

Altaf, A., SA Shah, and A. Memon. "Reducing the risk of injecting related harm in Karachi - a follow-up study. Draft Report. Marie Adelaide Habilitation Programme, Burns Road, Karachi.

Amon, et al. 2000. "Behavioral Surveillance Surveys BSS - Guidelines for repeated behavioral surveys in population at risk of HIV" by FHI, Impact, DFID.

Anderson, AF, Z Qingsi et al. 2003. "China's floating population and the potential for HIV transmission: a social-behavioral perspective." AIDS Care. 15: 177-195.

Aral and Van Dam. 1999. Cited in Cates, W. "STD Prevention in the US from Past to Present to Future." Presented at the National STD Prevention Conference. 2 March 2002. San Diego, California.

Asthana, S. and R. Oostvogels. 2001. "The social construction of male 'homosexuality' in India: implications for HIV transmission and prevention." Social Science and Medicine. 52: 707-721.

Auvert, B, D Taljaard, et al. 2005. "Randomized, controlled intervention trial of male circumcision for reduction of HIV infection risk: The ANRS 1265 trial." PloS Medicine. 2, 11: e298

A Pilot Study in Karachi and Rawalpindi 2004-05. Integrated Biological and Behavioral Surveillance. National AIDS Control Program, Ministry of Health and Canada - Pakistan HIV/AIDS Surveillance Project.

Auvert, B, A Buve, B Ferry, M Carael, L Morison, et al. 2001a. “Ecological and individual level analysis of risk factors for HIV infection in four urban populations in sub-Saharan Africa with different levels of HIV infection." AIDS. 15 (suppl 4): S15-S30. 
Auvert, B, A Buve, E Lagarde, M Kahindo, J Chege, N Rutenberg, et al. 2001b. “Male circumcision and HIV infection in four cities in sub-Saharan Africa." AIDS. 15 (suppl 4): S31-S40.

Baqi, S., SA Shah, et al. 1999. "Seroprevalence of HIV, HBV, and syphilis and associated risk behaviors in male transvestites (Hijras) in Karachi, Pakistan." International Journal of STD and AIDS. 10: 300-304.

Bentley, ME, K. Spratt, et al. 1998. “HIV testing and counseling among men attending sexually transmitted disease clinics in Pune, India: changes in condom use and sexual behavior over time." AIDS. 12: 1869-1877.

Benz, S. "The killing of the fittest- a quantitative analysis of HIV/AIDS and conflict." Paper prepared for DVPW Conference. Mannheim University, Germany, October 6-7, 2005.

Brinton LA, LI JY, Rang SD, et al. 1991. "Risk factors for penile cancer; results from a case-control study in China." International Journal of Cancer. 47; 50A-9

Brown, ZA, A Wald, RA Morrow et al. 2003. "Effect of serologic status and cesarean delivery on transmission rates of herpes simplex virus from mother to infant." JAMA. 289: 203-209.

Bryan, AD, JD Fisher, and TJ Benziger. 2001. “Determinants of HIV risk among Indian truck drivers." Social Science and Medicine. 53: 1413-1426.

Buve, A, E Lagard, M Carael, N Rutenberg, B Ferry et al. 2001. “Interpreting sexual behavior data: validity issues in the multicentre study on factors determining the differential spread of HIV in four African cities." AIDS. 15 (suppl 4): S117S126.

Caldwell, JC. 2006. "Will HIV/ AIDS levels in Asia reach the level of Sub-Saharan Africa." Asia-Pacific Population Journal. 21,1:3-9.

Caldwell, B. I Pieris and Barkat-e-Khuda et al. 1999. "Sexual regimes and sexual networking: the risk of an HIV/AIDS epidemic in Bangladesh." Social Science and Medicine. 48,8: 1103-1116.

Cameron DW, Simonsen JN, D'Costa LJ, Ronald AR, Maitha GM, Gakinya MN, et al. 1992. "Female to male transmission of human immunodeficiency virus type 1 risk factors for seroconversion in men." Lancet. 8660: 403-7.

Carael, M. et al., (1995) 'Sexual behaviour in developing countries: implications for HIV control’. AIDS 9: 1171-1175. 
Carael, M 1995, Sexual Behavior in J. Cleland and B, Ferry (EDS.), Sexual Behavior and AIDS in the Developing World, LONDON: World Health Organization, Taylor and Francis, PP75 - 121.

Carael, M., E Slaymaker, R. Lyerla and S Sarkar. 2006. “Clients of sex workers in different regions of the world: hard to count." Sexually Transmitted Infections. 200682 (Suppl 3):1-9.

Chandhiok, N. and RR Gangakhedkar. 2007. "Roundtable: The new evidence on male circumcision: An Indian perspective." Reproductive Health Matters. 15, 29: 53-56.

Chandrasekaran, P, G. Dallabetta, V. Loo et al. 2006. “Containing HIV/ AIDS in India: the unfinished agenda." Lancet Infectious Diseases. 6: 508-21.

Cheluget B, Joesoef MR, Marum LH, Wandera C, Ryan CA, DeCock KM, Chebet KL. 2004. "Changing patterns in sexually transmitted disease syndromes in Kenya after the introduction of a syndromic management program." Sexually Transmitted Diseases. 31,9: 522-525.

Chen, XS, YP Yin, et al. 2006. "Prevalence of sexually transmitted infections among long-distance truck drivers in Tongling, China." International Journal of STD and AIDS. 17: 304-308.

Choi, KH, DR. Gibson, L. Han, and Y. Guo. 2004. "High levels of unprotected sex with men and women among men who have sex with men: A potential bridge of HIV transmission in Beijing, China." AIDS Education and Prevention. 61,1: 19-30.

Cleland, J, JT Boerma, M Carael and SS Weir. 2004. "Monitoring sexual behavior in general population: a synthesis of lessons of the past decade." Sexually Transmitted Infections. 80: 1-7.

Coast, E. 2006. "Local understandings of, and responses to, HIV: Rural-urban migrants in Tanzania." Social Science and Medicine. 63,4: 1000-1010.

Collumbien, M and S. Hawkes. 2000. “Missing men's messages: does the reproductive health approach respond to men's sexual health needs?" Culture, Health, and Sexuality. 2,2: 135-150.

Dandona, L., R. Dandona, et al. 2005. "Sex behavior of men who have sex with men and risk of HIV in Andhra Pradesh, India." AIDS. 19:611-619.

DeJong, J. and G. El-Khoury. 2006. "Reproductive health of Arab young people.” BMJ. 333: 849-850. 
Douthwaite, MR and L Saroun. 2006. "Sexual behavior and condom use among unmarried young men in Cambodia." AIDS Care. 18,5: 505-513.

"Draft Report: Study of the Sexual Behaviors and Prevalence of STIs among 'Men on the Move' in Lahore, Pakistan." Submitted to London School of Hygiene and Tropical Medicine, April 2005 by Arjumand and Associates, Islamabad, Pakistan.

Emmanuel, F., S. Akhtar, A. Attarad and C. Kamran. 2004. "HIV risk behavior and practices among heroin addicts in Lahore, Pakistan."

Faisel, A. and J. Cleland. 2006. "Migrant men: a priority for HIV control in Pakistan?" Sexually Transmitted Infections. 82: 307-310.

Faisel, A. and J. Cleland. Study of the Sexual Behaviors and Prevalence of STIs Among Migrant Men in Lahore, Pakistan. December 2006. Conducted by Arjumand and Associates and Centre for Population Studies, LSHTM.

Family Health International. 2001. Summary Report: Behavioral Surveillance Survey in Healthy Highway Project, India. DFID and FHI.

Family Health International. 2007. Implementing AIDS Prevention and Care Project Pakistan Final Report. FHI: Arlington, VA.

Farrell, O, et al. 2006. "Association between HIV and subpreputial penile wetness in uncircumcised men in South Africa." JAIDS 2006, 43:69-77,117-118.

Filmer, D and L Pritchett. 1998. The effect of household wealth on educational attainment around the world: Demographic and Health Survey evidence. Washington, DC: The World Bank.

Franco EL, Campos Fiho N, Villa LL, et al. 1988. "Correlation patterns of cancer relative frequencies with some socio-economic and demographic indicators in Brazil: an ecologic study." International Journal of Cancer. 41: 24-9.

Furber, AS, R Maheswaran, JN Newell, and C Carroll. 2007. “Is smoking tobacco an independent risk factor for HIV infection and progression to AIDS? A systematic review." Sexually Transmitted Infection. 83: 41-46.

Ghauri, AK and SA Shah, MA Memom, "Follow-up study to evaluate change in KABP and sero-prevalence of HIV, HBV, HCV and syphilis among IDUs at DIC, Essa Nagri, Karachi. UNODC/UNAIDS, Islamabad. 
Gibney, L, P Choudhury, Z Khawaja, M Sarker, and SH Vermund. 1999. “Behavioral risk factors for HIV/ AIDS in a low-HIV prevalence Muslim nation: Bangladesh." International Journal of STD and AIDS. 10: 186-194.

Gibney, L. N Squib and J Metzger. 2003. “Behavioral risk factors for STD/HIV transmission in Bangladesh's trucking industry." Social Science and Medicine. 56,7: 1411-1424.

Girault, P, T Saidel, N Song, et al. 2004. "HIV, STIs, and sexual behaviors among men who have sex with men in Phnom Penh, Cambodia." AIDS Education and Prevention. 16,1: 31-44.

Godwin, P, N O-Farrel, K Fylkesnes, and S. Misra. 2006. "Five myths about the HIV epidemic in Asia." PloS Medicine. 3,10: e426.

Gomes do Espirito Santo, ME, and GD Etheredge. 2005. "Male clients of brothel prostitutes as a bridge for HIV infection between high risk and low risk groups of women in Senegal." Sexually Transmitted Infections. 81: 342-344.

Gorbach, PM, H Sopheab, T Phalla, HB Leng et al. 2000. “Sexual bridging by Cambodian men: Potential importance for general population spread of STD and HIV epidemics." Sexually Transmitted Diseases. 27,6: 320-326.

Gray, PB. 2004. "HIV and Islam: is HIV prevalence lower among Muslims?" Social Science and Medicine. 58: 1751-1756.

Gray, RH. Et al. 2001. "Probability of HIV-1 transmission per coital act in monogamous, heterosexual, HIV-1-discordant couples in Rakai, Uganda." Lancet. 357,9263: 1149-1153.

Halperin DT, Bailey RC. 1999. Male circumcision and HIV infection: 10 years and counting. Lancet. 354:1813-1815

Haque, N, T Zafar, H Brahmbhatt et al. 2004. “High-risk sexual behaviors among drug users in Pakistan: implications for prevention of STDS and HIV/ AIDS." International Journal of STD and AIDS. 15: 601-07.

Hawkes, S, L. Morison, J. Chakraborty et al. 2002. "Reproductive tract infections: prevalence and risk factors in rural Bangladesh." Bulletin of the World Health Organization. 80,3: 180-188.

Hawkes, S and KG Santhya. 2001. “Diverse realities: sexually transmitted infections and HIV in India." Population Council Regional Working Paper Series. New Delhi: Population Council. 
Hawkes, S., L. Morison, S. Foster, et al. 1999. “Managing RTIs in women in low prevalence, low income situations: an evaluation of syndromic management in Matlab, Bangladesh." Lancet. 354: 1776-81.

Hawkes, S. and GJ Hart. 1993. “Travel, Migration and HIV.” AIDS Care. 5,2: 207-214.

Hawkes, S., S. Pachauri, and P. Mane. 2002. "Editorial introduction" Culture, Health and Sexuality. 4,2: 125-131.

Hernandez, AL, CP Lindan, M. Mathur et al. 2006. “Sexual behavior among men who have sex with women, men, and Hijras in Mumbai, India - multiple sexual risks." AIDS Behavior. DOI 10.1007?s10461-006-9129-z.

HIV Seroprevalence Study Group of Pakistan, National AIDS Programme. 1996. “HIV seroprevalence surveys in Pakistan." AIDS. 10,8: 926-27.

HIV Seroprevalence Study Group of Pakistan. 1996. “HIV seroprevalence surveys in Pakistan." AIDS. 10,8: 926-27.

Hong, Y., B. Stanton, X. Li, et al. 2006. “Rural-to-Urban migrants and the HIV epidemic in China." AIDS and Behavior.

Hor, LB, R Detels, S Heng, and P Mun. 2005. "The role of sex worker clients in transmission of HIV in Cambodia." International Journal of STDs and AIDS. 16,2: 170-174.

Hyder, AA, and OA Khan. 1998. "HIV/ AIDS in Pakistan: the context and magnitude of an emerging threat." Journal of Epidemiology and Community Health. 52: 579585.

Kasubi, MJ, A Nilsen, HS Marsden, T Bergstrom, N Langeland, and L Haarr. 2006. "Prevalence of antibodies against Herpes Simplex Virus Types 1 and 2 in children and young people in an urban region of Tanzania." Journal of Clinical Microbiology. 44,8: 2801-2807.

Kelley, LM and N Eberstadt. 2005. Behind the Veil of a Public Health Crisis: HIV/AIDS in the Muslim World. National Bureau of Asian Research Special Report. Seattle, Washington: National Bureau of Asian Research.

Khan, AA. 2005. "HIV/AIDS: The State of the Nation." Infectious Diseases Journal of Pakistan. 64-65.

Khan, AA. 2005. "Letter to the Editor: HIV testing in low prevalence populations." Journal of Pakistan Medical Association. 55,9: 406. 
Khan, I., et al. 1995. "Pattern of sexually transmitted diseases in Hazara division." JAMC (Journal of Ayub Medical College) 7(2): 28-9.

Khan, OA, and AA Hyder. 1998. "HIV/AIDS among men who have sex with men in Pakistan." Sexual Health Exchange. No. 2.

Khan, S. and OA Khan, 2006. "Letter: The trouble with MSM" American Journal of Public Health. 96,5: 765-766.

Khan, S. and T. Khilji. 2002. "Pakistan Enhanced HIV/ AIDS Program: Social Assessment and Mapping of Men who have Sex with Men (MSM) in Lahore, Pakistan." Short Report for the World Bank, Pakistan prepared by Naz Foundation International.

Khandwalla, HE, S. Luby, and S. Rahman. 2000. “Knowledge, attitudes, and practices regarding sexually transmitted infections among general practitioners and medical specialists in Karachi, Pakistan." Sexually Transmitted Infections. 76:383385.

Khokhar, O., G. Javaid Malik, and N. Khokhar. 2003. "Prevalence of HIV infection in a health population in Northern Pakistan." Rawal Medical Journal. 28,1:12-16.

Khushk, IA and M Masood Kadir. 2006. “Editorial: HIV/AIDS threat in Pakistan: Changing concerns and realities." JCPSP. 16,10: 631-632.

Kongnyuy, EJ, CS Wiysonge, et al. 2006. "Wealth and sexual behavior among men in Cameroon." BMC International Health and Human Rights. 6,11.

Kumar, R., P. Jha, P. Arora, et al. 2006. “Trends in HIV-1 in young adults in south India from 2000 to 2004: a prevalence study." Lancet. 367: 1164-1172.

LaGarde, E., M Carael, J Glynn et al. 2001. “Educational level is associated with condom use within non-spousal partnerships in four cities of sub-Saharan Africa." AIDS. 15,11: 1399-1408.

Lalou, R and V Piche. 2002. “Migrants and AIDS: Risk management versus social control. An example from the Senegal River Valley." Population-E. 59,2:195-228.

Lau, JTF and J. Thomas. 2001. “Risk behaviors of Hong Kong male residents traveling to mainland China: a potential bridge population for HIV infection." AIDS Care. 13,1: 71-81. 
Lau, JTF, JH Kim, M Lau and HY Tsui. 2004. "HIV related behaviors and attitudes among Chinese men who have sex with men in Hong Kong: a population based study." Sexually Transmitted Infections. 80:459-465.

Liu, JX, and K. Choi. 2006. "Experiences of social discrimination among men who have sex with men in Shanghai, China." AIDS Behavior. 10: S25-S33.

Liu, H., J. Xie, W.Yu et al. 1998. “A study of sexual behavior among rural residents of China." Journal of Acquired Immune Deficiency Syndrome and Human Retrovirology. 19,1: 80-8.

Liu, H, O Grusky, et al. 2006. "Drug users: a potentially important bridge population in the transmission of sexually transmitted diseases, including AIDS, in China." Sexually Transmitted Diseases. 33,2: 111-7.

Lowndes, CM, M Alary, H Meda, CAB Gnintoungbe et al. 2002. "Role of core and bridging groups in the transmission dynamics of HIV and STIs in Cotonou, Benin, West Africa." Sexually Transmitted Infection. 78: i69-i77.

LSHTM, Population Council, and AKU. 2002. National Study of Reproductive Tract Infections and Sexually Transmitted Infections. Annexe: Proposed Research Strategy and Study Design.

Luby SP, Qamruddin K, Shah AA et al. 1997. “The relationship between therapeutic injections and high prevalence of hepatitis C infections in Hafizabad, Pakistan." Epidemiology infect. 119: 349-356

Malta, M, FI Bastos, et al. 2006. “A qualitative assessment of long distance truck drivers' vulnerability to HIV/ AIDS in Itajai, southern Brazil." AIDS Care. 18,5: 489-496.

Mann, J and D Tarantola. 1996. AIDS in the World II. Schwartz.

Marfatia, YS, S Archana, et al. 2005. "Health care seeking behavior of STD patients." Indian Journal of Sexually Transmitted Diseases. 26,1: 16-18.

Marston, C. and E. King. 2006. “Factors that shape young people's sexual behavior: A systematic review." Lancet. 368: 1581-1586.

Mazar, SB, MA Agha, MA Shaikh. 2001. “Knowledge and misconceptions about sexually transmitted infections in married women - perspective from Islamabad." Journal of the Pakistan Medical Association. 51: 389-92. 
Mishar, V, M Vaessen, JT Boerma et al. 2006. “HIV testing in national population-based surveys: experience from the Demographic and Health Surveys." Bulletin of the World Health Organization. 84,7: 537-545.

Mishra, V., M. Vaessen, J. Ties Boerma, F. Arnold, A. Way, B. Barrere, A. Cross. R. Hong, and J. Sangha. 2006. "HIV testing in national population-based surveys: Experience from the Demographic and Health Surveys." Bulletin of the World Health Organization. 84,7: 537-545.

Mitra, P. 2004. "India at the crossroads: Battling the HIV/AIDS pandemic." The Washington Quarterly. 27,4: 95-107.

Moodley, P, Strum PD, Vanmali T, Wilkinson D, Connolly C, Strum AW. 2003. "Association between HIV-1 infection, the etiology of genital ulcer disease and response to syndromic treatment." Sexually Transmitted Diseases. 2003:30,3:2415.

Morisky, DE, C Nguyen, A Ang, and TV Tiglao. 2005. “HIV/AIDS prevention among the male population: results of a peer education program for taxicab and tricycle drivers in the Philippines." Health Education and Behaviors. 32,1: 57-68.

Morris, M, C Podhisita, et al. 1996. “Bridge populations in the spread of HIV/AIDS in Thailand." AIDS. 10,11: 1265-71.

Mujeeb, SA, K Aamir, K Mehmood. 2000. "Seroprevalence of HBV, HCV and HIV infections among college going first time voluntary blood donors." Journal of the Pakistan Medical Association. 50: 269-70.

National Study of Reproductive Tract and Sexually Transmitted Infections. Survey of High Risk Groups in Lahore and Karachi, 2005. National AIDS Control Program (NACP), Government of Pakistan, FHI and DFID.

Nanan, DJ, MM Kadir, and FMM White. 2000. “Survey and surveillance development in settings with low human immuniodeficiency virus prevalence." Eastern Mediterranean Health Journal. 6,4: 67-77.

Nessa, K., S. Waris, Z. Sultan, S. Monira, M. Hossain, S. Nahar, H. Rahman, M. Alam, P. Baatsen, and M. Rahman. 2004. "Epidemiology and etiology of sexually transmitted infection among hotel-based sex workers in Dhaka, Bangladesh." Journal of Clinical Microbiology. 42,2: 618-621.

Nicolosi, A., D.B. Glasser, S. C. Kim, et al. 2005. "Sexual behavior and dysfunction and help-seeking patterns in adults aged 40-80 years in the urban population of Asian countries." BJU International. 95: 609-614. 
Nishimura, YH, M Ono-Kihara, JC Mohith et al. 2007. "Sexual behaviors and their correlates among young people in Mauritius: a cross-sectional study." BMC International Health and Human Rights. 7,8: doi.10.1186/1472-698X-7-8.

Obermeyer, CM. 2006. “HIV in the Middle East.” BMJ. 333: 851-854.

O'Connor, CC, LM Wen, C Rissel and M Shaw. 2007. "Sexual behavior and risk in Vietnamese men living in metropolitan Sydney." Sexually Transmitted Infections. 83: $147-150$.

O'Farrell, N. 1993. "Soap and water prophylaxis for limiting genital ulcer disease and HIV-1 infection in men in sub-Saharan Africa." Genitourinary Medicine. 69: 297300.

Pakistan Census Organization, 1988.

PSLM Pakistan Social and Living Standards Measurement Survey, Govt. of Pakistan 2004-2005.

Panchanadeswaran, S., SC Johnson, K. Mayer et al. 2006. “Gender differences in STI prevalence and genital symptoms in an urban setting in Southern India." Sexually Transmitted Infection. Online published June 6, 2006 as 10.1136/sti.2006.020768.

Parker, RG, D. Easton, and CH Klein. 2000. "Structural barriers and facilitators in HIV prevention: a review of international research." AIDS. 14 Supplement 1: S22S32.

Perera, B. and M. Reece. 2006. "Sexual behavior of young adults in Sri Lanka: Implications for HIV prevention." AIDS Care. 18,5: 497-500.

Pettifor, AE, HV Rees, A Steffenson et al. 2004a. HIV and Sexual Behavior Among Young South Africans: A National Survey of 15-24 year olds. Johannesburg: Reproductive Health Research Unit. University of the Witwatersrand.

Pettifor, AE, A van der Straten, M Dunbar et al. 2004b. “Early age of first sex: a risk factor for HIV infection among women in Zimbabwe." AIDS. 18,10: 1435-1442.

Poulin, M. "Sex, money, and premarital partnerships in southern Malawi." Social Science and Medicine. Article in press.

Puri, M. and J. Cleland. 2006. "Sexual behavior and perceived risk of HIV/AIDS among young migrant factory workers in Nepal." Journal of Adolescent Health. 38:237-246. 
Qian, ZH, SH Vermund, N Wang. 2005. “Risk of HIV/ AIDS in China: subpopulations of special importance." Sex Transm Infect. 81: 442-447.

Raglow GJ, Luby SP, Nabi N. Therapeutic injections in Pakistan: from the patients, perspective, Trop Med Int Health 2001; 6: 69-75.

Rao, KS, RD Pilli, AS Rao, and PS Chalam. 2006. "Sexual lifestyle of long distance lorry drivers in India: questionnaire survey." BMJ. 318: 162-163.

Rehan, N. 2006. "Profile of men suffering from sexually transmitted infections in Pakistan." Journal of the Pakistan Medical Association. 56, 1: S60-S65.

Rivers, K. and P. Aggleton. 1999. “Men and the HIV Epidemic.” UNDP HIV and Development Programme.

Rodrigues, JJ, SM Mehendale, ME Shepard, et al. 1995. “Risk factors for HIV infection in people attending clinics for sexually transmitted diseases in India." BMJ. 311: 283-286.

Roudi-Fahimi, F. 2007. Time to Intervene: Preventing the Spread of HIV/AIDS in the Middle East and North Africa. Washington, DC: Population Reference Bureau.

Rutstein, SO and K Johnson. 2004. The DHS Wealth Index. DHS Comparative Reports No. 6. Calverton, Maryland: ORC Macro.

Schensul, SL, A. Mekki-Berrada, et al. 2006a. “Healing traditions and men's sexual health in Mumbai, India: The realities of practiced medicine in urban poor communities." Social Science and Medicine. 62: 2774-2785.

Schensul, SL, A Mekki-Berrada, B. Nastasi et al. 2006b. “Men's extramarital sex, marital relationships and sexual risk in urban poor communities in India." Journal of Urban Health.

Shah, SA, and A. Altaf. "Editorial: Prevention and control of HIV/ AIDS among injection drug users in Pakistan: A great challenge."

Shah, SA, A. Altaf, SA. Mujeeb, and A. Memon. "An outbreak of HIV infection among injection drug users in a small town in Pakistan: potential for national implications." Page 209.

Shah, SA, OA Khan, S. Kristensen and SH Vermund. 1999. "HIV-infected workers deported from the Gulf States: impact on Southern Pakistan." International Journal of STD and AIDS. 10: 812-814. 
Shelton, J. 2007. “Ten myths and one truth about generalized HIV epidemics.” Lancet. 370: 1809-1811.

Silverman, JG, MR Decker, NA Kapur, J Gupta and A Raj. 2007. “Violence against wives, sexual risk and sexually transmitted infection among Bangladeshi men." Sexually Transmitted Infections. 83: 211-215.

Slaymaker, E. N. Walker, B. Zaba and M Collumbien. Chapter 14, “Unsafe Sex” in Ezzati et al. 2006.

Sopheab, H., K. Fylkesnes, et al. 2006. "HIV-related risk behaviors in Cambodia and effects of mobility." Journal of Acquired Immune Deficiency Syndrome. 41,1: 81-86.

Stamm WE, Handsfiedl HH, Rempals A et al. 1988. "The association between genital ulcer disease and acquisition of HIV infection in homosexual men." JAMA. 260:1429-33

Steele, MS, E Bukusi, CR Cohen, BA Shell-Duncan and KK Holmes. 2004. "Male genital hygiene beliefs and practices in Nairobi, Kenya." Sexually Transmitted Infections. 80: $471-476$.

Stein, JA and A Nyamathi. 2000. "Gender differences in behavioral and psychological predictors of HIV testing and return for test results in a high-risk population." AIDS Care. 12,3: 343-356.

Sudha, RT, DT Vijay, V. Lakshmi. 2005. “Awareness, attitudes and beliefs of the general public towards HIV / AIDS in Hyderabad, a capital city from South India." Indian Journal of Medical Sciences. 59,7: 307-316.

Swidler, A. and S. Cotts Watkins. 2007. "Ties of dependence: AIDS and transactional sex in Rural Malawi." Studies in Family Planning. 38,3: 147-162.

Thomas, B., F. Rahman et al. 2004. "A study of condom acceptability among men in an urban population in South India." AIDS and Behavior. 8,2: 215-220.

Trollope-Kumar, K and G. Guyatt. 2006. “Syndromic approach for treatment of STIs: time for a change." Lancet. 367: 1380-81.

Tucker, JD, GE Henderson, TF Wang, YY Huang, W Parish et al. 2005. “Surplus men, sex work, and the spread of HIV in China." AIDS. 19,6: 539-547.

Valleroy, LA, DA MacKellar, et al. 2000. "HIV prevalence and associated risks in young men who have sex with men." JAMA. 284,2: 198-204. 
Verma, RK and M Collumbien. "Wife beating and the link with poor sexual health and risk behavior among men in urban slums in India." Journal of Comparative Family Studies.

Verma, V. et al. 1980 Sample designs and sampling errors for the World Fertility Survey. Journal of the Royal Statistical Society A.143(40:431-63.

Wald A, Link K. 2002. "Risk of human immunodeficiency versus infection in herbs simplex virus type 2 sero positive persons: a meta analysis." J Infect Dis 185: 45 $-52$.

Wang Q, Yang P, Zhong M, Wang G, Validation of diagnostic algorithms for syndromic management of sexually transmitted diseases Chinese Medical Journal (English) 2003;116(2):181-6 Wardlow, H. 2007. “Men's extramarital sexuality in Rural Papua New Guinea." American Journal of Public Health. 97,6: 1006-1014.

Weiser, SD, K Leiter, M Heisler, W McFarland et al. 2006. “A population-based study on alcohol and high-risk sexual behaviors in Botswana." PloS Medicine. 3,10. DOI: 10.1371/journal.pmed.0030392.

Williams, BG, JO Lloyd-Smith, et al. 2006. "The potential impact of male circumcision on HIV in Sub-Saharan Africa." PloS Medicine. 3,7: e262.

World Bank - http://go.worldbank.org/PJHKZAQHGO

Yang, X and G Xia. 2006. "Gender, migration, risky sex, and HIV infection in China." Studies in Family Planning. 37,4: 241-250.

Zafar, T., H. Brahmbatt, et al. 2003. "HIV knowledge and risk behaviors among Pakistani and Afghani drug users in Quetta, Pakistan." JAIDS. 32: 394-398.

Zhao, M, QY Wang, GH Lu, et al. 2005. “Risk behaviors and HIV/AIDS prevention education among IDUs in drug treatment in Shanghai." Journal of Urban Health: Bulletin of the New York Academy of Science. 82,3: iv84-iv91.

Zuma, K, MN Lurie, BG Williams et al. 2005. “Risk factors of sexually transmitted infections among migrant and non-migrant sexual partnerships from rural South Africa." Epidemiol. Infect. 133: 421-428. 
APPENDICES 



\section{APPENDIX 1: CITY WISE DETAILS OF RESPONDENTS}

Table A1.1: Age, by city

\begin{tabular}{lrrrrrr}
\hline Age group & Faisalabad & Karachi & Lahore & Peshawar & Quetta & Rawalpindi \\
\hline $16-20$ years & 15.8 & 21.5 & 17.8 & 20.5 & 18.3 & 22.3 \\
$21-25$ years & 27.0 & 24.5 & 19.8 & 25.3 & 21.0 & 25.5 \\
$26-30$ years & 14.8 & 21.8 & 18.0 & 15.8 & 18.8 & 17.5 \\
$31-35$ years & 12 & 13.0 & 14.3 & 11.3 & 10.0 & 10.0 \\
$36-40$ years & 15 & 8.8 & 12.8 & 13.3 & 15.0 & 12.0 \\
$41-45$ years & 15.5 & 10.5 & 17.5 & 14.0 & 17.0 & 12.8 \\
\hline Mean age in years & 29.6 & 27.9 & 30 & 28.8 & 30 & 28.2 \\
Median age in years & 28 & 26 & 29 & 27 & 29 & 26 \\
\hline
\end{tabular}

Table A1.2: Education, by city

\begin{tabular}{lrrrrrr}
\hline Education & Faisalabad & Karachi & Lahore & Peshawar & Quetta & Rawalpindi \\
\hline No education & 17.8 & 16.3 & 15.8 & 17.5 & 15.0 & 7.0 \\
Up to secondary & 56.1 & 57.1 & 59.3 & 48.6 & 55.0 & 43.3 \\
Above secondary & 26.3 & 26.8 & 25 & 34.0 & 30.0 & 49.8 \\
\hline
\end{tabular}

Table A1.3: Employment status, by city

\begin{tabular}{lrrrrrr}
\hline Employment status & Faisalabad & Karachi & Lahore & Peshawar & Quetta & Rawalpindi \\
\hline Unemployed & 3.8 & 3.0 & 3.0 & 3.0 & 3.8 & 1.3 \\
Student & 11.3 & 10.8 & 12.3 & 20.3 & 16.3 & 29.8 \\
Employed & 85.0 & 86.3 & 84.8 & 76.8 & 80.0 & 69.0 \\
Number of respondents & $\mathbf{4 0 0}$ & $\mathbf{4 0 0}$ & $\mathbf{4 0 0}$ & $\mathbf{4 0 0}$ & $\mathbf{4 0 0}$ & $\mathbf{4 0 0}$ \\
\hline
\end{tabular}

Table A1.4: Details of employment, by city

\begin{tabular}{lrrrrrr}
\hline Type of employment & Faisalabad & Karachi & Lahore & Peshawar & Quetta & Rawalpindi \\
\hline Government service & 11.2 & 11.3 & 10.0 & 33.9 & 29.1 & 23.2 \\
Private service & 57.9 & 52.5 & 31.9 & 37.1 & 20.9 & 35.1 \\
Self employed & 30.9 & 36.2 & 58.1 & 29.0 & 50.0 & 41.7 \\
Number of respondents & $\mathbf{3 4 0}$ & $\mathbf{3 4 5}$ & $\mathbf{3 3 9}$ & $\mathbf{3 0 7}$ & $\mathbf{3 2 0}$ & $\mathbf{2 7 6}$ \\
\hline
\end{tabular}


Table A1.5: Marital status of respondents, by city

\begin{tabular}{lrrrrrr}
\hline Marital status & Faisalabad & Karachi & Lahore & Peshawar & Quetta Rawalpindi \\
\hline Unmarried & 45.5 & 53.5 & 45.0 & 43.0 & 41.5 & 57.0 \\
Married & 53.0 & 45.3 & 54.8 & 57.0 & 57.8 & 42.8 \\
Formerly married & 1.5 & 1.3 & 0.3 & 0.0 & 0.8 & 0.3 \\
Total & 100.0 & 100.0 & 100.0 & 100.0 & 100.0 & 100 \\
Number of respondents & $\mathbf{4 0 0}$ & $\mathbf{4 0 0}$ & $\mathbf{4 0 0}$ & $\mathbf{4 0 0}$ & $\mathbf{4 0 0}$ & $\mathbf{4 0 0}$ \\
\hline
\end{tabular}

Table A1.6: Language of respondents, by city

\begin{tabular}{lrrrrrrr}
\hline Language & Faisalabad & Karachi & Lahore Peshawar & Quetta Rawalpindi & Overall \\
\hline Punjabi & 89.8 & 16.8 & 77.8 & 8.3 & 11.3 & 59.0 & 43.8 \\
Pashto & 0.3 & 16.5 & 5.0 & 77.3 & 38.3 & 5.8 & 23.8 \\
Urdu & 8.0 & 32.5 & 15.0 & 3.8 & 3.5 & 21.0 & 14.0 \\
Other $^{\text {a }}$ & 2.1 & 34.4 & 2.4 & 10.9 & 47.2 & 14.3 & 18.4 \\
Number of respondents & $\mathbf{4 0 0}$ & $\mathbf{4 0 0}$ & $\mathbf{4 0 0}$ & $\mathbf{4 0 0}$ & $\mathbf{4 0 0}$ & $\mathbf{4 0 0}$ & $\mathbf{2 , 4 0 0}$ \\
\hline
\end{tabular}

a Includes Sindhi, Balochi, Siraiki, Brahvi, Hindko, and Farsi.

Table A1.7: Self-reported monthly income, by city

\begin{tabular}{lrrrrrr}
\hline Monthly income & Faisalabad & Karachi & Lahore & Peshawar & Quetta & Rawalpindi \\
\hline Rs. 1,000-5,000 & 44.6 & 39.4 & 37.0 & 51.2 & 35.4 & 27.7 \\
Rs. 5,001-15,000 & 45.2 & 53.0 & 56.6 & 39.3 & 53.8 & 52.8 \\
Rs. 15,001 and above & 10.1 & 7.7 & 6.4 & 9.5 & 10.8 & 19.5 \\
Number of respondents & $\mathbf{4 0 0}$ & $\mathbf{4 0 0}$ & $\mathbf{4 0 0}$ & $\mathbf{4 0 0}$ & $\mathbf{4 0 0}$ & $\mathbf{4 0 0}$ \\
\hline
\end{tabular}

Table A1.8: Living arrangements, by city

\begin{tabular}{lrrrrrr}
\hline Living arrangement & Faisalabad & Karachi & Lahore & Peshawar & Quetta & Rawalpindi \\
\hline Parents & 46.0 & 42.8 & 47.0 & 39.5 & 40.8 & 42.5 \\
Parents and wife & 26.5 & 15.8 & 23.5 & 26.0 & 25.3 & 14.0 \\
Wife & 22.8 & 16.0 & 24.8 & 26.0 & 28.3 & 19.0 \\
Other & 4.8 & 25.5 & 4.8 & 8.5 & 5.8 & 24.5 \\
Number of respondents & $\mathbf{4 0 0}$ & $\mathbf{4 0 0}$ & $\mathbf{4 0 0}$ & $\mathbf{4 0 0}$ & $\mathbf{4 0 0}$ & $\mathbf{4 0 0}$ \\
\hline
\end{tabular}


Table A1.9: Personal experience with Ulcer/Sores

\begin{tabular}{|c|c|c|c|c|c|c|}
\hline & Faisalabad & Karachi & Lahore & Peshawar & Quetta & Rawalpindi \\
\hline \multicolumn{7}{|l|}{ Ulcer/Sores in genital area } \\
\hline Yes & 3.3 & 2.8 & 1.3 & 3.3 & 0.5 & 1 \\
\hline No & 96.8 & 97.3 & 98.8 & 96.8 & 99.5 & 99 \\
\hline Number of respondents & 400 & 400 & 400 & 400 & 400 & 400 \\
\hline \multicolumn{7}{|c|}{ Did you seek treatment for ulcer/sores in genital area } \\
\hline Yes & 84.6 & 72.7 & 100 & 69.2 & 50 & 50 \\
\hline No & 15.4 & 27.3 & & 30.8 & 50 & 50 \\
\hline Number of respondents & 13 & 11 & 5 & 13 & 2 & 4 \\
\hline \multicolumn{7}{|c|}{ Where did you seek treatment } \\
\hline Doctor & 54.5 & 87.5 & 60 & 55.6 & 100 & 50 \\
\hline Dispenser/Paramedic & 18.2 & 0 & 0 & 0 & 0 & 0 \\
\hline Traditional Practitioners & 36.4 & 37.5 & 20 & 33.3 & 100 & 50 \\
\hline Self medication & 9.1 & 12.5 & 20 & 0 & 0 & 0 \\
\hline Others & 0 & 0 & 0 & 11.1 & 0 & 0 \\
\hline Number of respondents & 11 & 8 & 5 & 9 & 1 & 2 \\
\hline \multicolumn{7}{|c|}{ Why did you not seek treatment } \\
\hline Not aware & 50 & 0 & 0 & 50 & 0 & 0 \\
\hline Facility not available & 50 & 0 & 0 & 25 & 0 & 0 \\
\hline Financial barriers & 50 & 0 & 0 & 0 & 0 & 0 \\
\hline Shyness & 50 & 66.7 & 0 & 0 & 0 & 0 \\
\hline Other & 0 & 33.3 & 0 & 75 & 0 & 100 \\
\hline No Information & 0 & 0 & 0 & 0 & 100 & 0 \\
\hline Number of respondents & 2 & 3 & 0 & 4 & 0 & 2 \\
\hline \multicolumn{7}{|c|}{ What was the problem diagnosed as } \\
\hline Problem not diagnosed & 9.1 & 12.5 & 40 & 44.4 & 0 & 0 \\
\hline Syphillis (Aatshak) & 0 & 25 & 0 & 0 & 0 & 0 \\
\hline \multirow[t]{2}{*}{ Other } & 90 & 71.4 & 33.3 & 50 & 100 & 100 \\
\hline & 18.2 & 12.5 & 40.0 & 0 & 0 & 50.0 \\
\hline Number of respondents & 10 & 7 & 3 & 10 & 1 & 3 \\
\hline \multicolumn{7}{|c|}{ How was the problem diagnosed } \\
\hline History/ sign symptoms & 90 & 85.7 & 100 & 87.5 & 100 & 50 \\
\hline Lab test & 10 & 0 & 0 & 0 & 0 & 0 \\
\hline Other (specify) & 0 & 14.3 & 0 & 12.5 & 0 & 50 \\
\hline Number of respondents & 10 & 7 & 2 & 8 & 1 & 4 \\
\hline \multicolumn{7}{|c|}{ Was your partner aware about your disease } \\
\hline Yes & 45.5 & 25 & 50 & 55.6 & 100 & 33.3 \\
\hline No & 54.5 & 75 & 50 & 44.4 & 0 & 66.7 \\
\hline Number of respondents & 11 & 8 & 4 & 9 & 1 & 3 \\
\hline
\end{tabular}




\begin{tabular}{|c|c|c|c|c|c|c|}
\hline & Faisalabad & Karachi & Lahore & Peshawar & Quetta & Rawalpindi \\
\hline \multicolumn{7}{|c|}{ What preventive measure did you use to prevent your partner } \\
\hline Nothing & 69.2 & 45.5 & 40 & 38.5 & 50 & 70 \\
\hline Avoid intercourse & 23.1 & 18.2 & 20 & 15.4 & 0 & 25 \\
\hline Used condom & 0 & 0 & 0 & 7.7 & 0 & 0 \\
\hline Never had sex & 7.7 & 9.1 & 20 & 7.7 & 0 & 0 \\
\hline Other (specify) & 23.1 & 25 & 0 & 11.1 & 0 & 25 \\
\hline No Information & 0 & 27.3 & 20.0 & 30.8 & 50.0 & 0 \\
\hline Number of respondents & 13 & 8 & 4 & 9 & 1 & 4 \\
\hline
\end{tabular}

Table A1.10: Personal experience with Purulent discharge

\begin{tabular}{|c|c|c|c|c|c|c|}
\hline & Faisalabad & Karachi & Lahore & Peshawar & Quetta & Rawalpindi \\
\hline \multicolumn{7}{|c|}{ Purulent discharge from penis } \\
\hline Yes & 1 & 5 & 1.5 & 0.8 & 1.8 & 2 \\
\hline No & 99 & 95 & 98.5 & 99.3 & 98.3 & 98 \\
\hline Number of respondents & 400 & 400 & 400 & 400 & 400 & 400 \\
\hline \multicolumn{7}{|c|}{ Did you seek treatment for purulent discharge from penis } \\
\hline Yes & 50 & 65 & 83.3 & 66.7 & 85.7 & 62.5 \\
\hline No & 50 & 35 & 16.7 & 33.3 & 14.3 & 37.5 \\
\hline Number of respondents & 4 & 20 & 6 & 3 & 7 & 7 \\
\hline \multicolumn{7}{|c|}{ Where did you seek treatment } \\
\hline Doctor & 100 & 76.9 & 20 & 100 & 60 & 0 \\
\hline Dispenser/Paramedic & 0 & 0 & 0 & 0 & 0 & 20 \\
\hline Traditional Practitioner & 0 & 53.8 & 100 & 50 & 60 & 100 \\
\hline Self Medication & 0 & 0 & 0 & 0 & 0 & 20 \\
\hline Others & 0 & 0 & 0 & 0 & 0 & 0 \\
\hline Number of respondents & 2 & 13 & 5 & 2 & 5 & 5 \\
\hline \multicolumn{7}{|c|}{ Why did you not seek treatment } \\
\hline Facility not available & 0 & 14.3 & 0 & 0 & 50 & 0 \\
\hline Financial barriers & 0 & 14.3 & 100 & 0 & 0 & 0 \\
\hline Shyness & 0 & 14.3 & 0 & 100 & 50 & 50 \\
\hline Other & 100 & 71.4 & 0 & 100 & 0 & 50 \\
\hline No Information & 0 & 0 & 0 & 0 & 0 & 33.0 \\
\hline Number of respondents & 2 & 7 & 1 & 1 & 2 & 2 \\
\hline \multicolumn{7}{|c|}{ What was the problem diagnosed as } \\
\hline Problem not diagnosed & 0 & 46.7 & 33.3 & 100 & 0 & 20 \\
\hline Gonorrhea (Sozaak) & 50 & 0 & 0 & 0 & 50 & 20 \\
\hline Syphillis (Aatshak) & 0 & 6.7 & 0 & 0 & 0 & 0 \\
\hline Hepatitis & 0 & 0 & 0 & 0 & 16.7 & 0 \\
\hline Other & 50 & 46.7 & 66.7 & 0 & 33.3 & 60 \\
\hline Number of respondents & 2 & 15 & 6 & 2 & 6 & 5 \\
\hline
\end{tabular}




\begin{tabular}{|c|c|c|c|c|c|c|}
\hline & Faisalabad & Karachi & Lahore & Peshawar & Quetta & Rawalpindi \\
\hline \multicolumn{7}{|c|}{ What was the problem diagnosed as } \\
\hline History/ sign symptoms & 50 & 71.4 & 83.3 & 0 & 100 & 75 \\
\hline Lab test & 50 & 28.6 & 0 & 100 & 0 & 0 \\
\hline Others & 0 & 0 & 16.7 & 0 & 0 & 25 \\
\hline Number of respondents & 2 & 7 & 6 & 1 & 5 & 4 \\
\hline \multicolumn{7}{|c|}{ Was your partner aware about your disease } \\
\hline Yes & 0 & 13.3 & 0 & 0 & 33.3 & 33.3 \\
\hline No & 100 & 86.7 & 100 & 100 & 66.7 & 66.7 \\
\hline Number of respondents & 2 & 15 & 4 & 2 & 6 & 3 \\
\hline \multicolumn{7}{|c|}{ What preventive measure did you use to prevent your partner } \\
\hline Nothing & 75 & 64.7 & 66.7 & 66.7 & 57.1 & 37.5 \\
\hline Avoid intercourse & 25 & 0 & 16.7 & 0 & 14.3 & 12.5 \\
\hline Used condom & 0 & 10 & 0 & 0 & 14.3 & 0 \\
\hline Never had sex & 0 & 5 & 0 & 33.3 & 0 & 12.5 \\
\hline Partner's treatment & 0 & 5 & 0 & 0 & 0 & 0 \\
\hline Others & 25 & 11.8 & 0 & 0 & 0 & 20 \\
\hline No Information & 0 & 15.0 & 16.7 & 0 & 14.3 & 37.5 \\
\hline Number of respondents & 4 & 17 & 5 & 3 & 6 & 5 \\
\hline
\end{tabular}

Table A1.11: Personal experience with Pain during urination

\begin{tabular}{lrrrrrr}
\hline & Faisalabad & Karachi & Lahore & Peshawar & Quetta & Rawalpindi \\
\hline Pain during urination & & & & & & \\
Yes & 3.3 & 5 & 2.5 & 3 & 4.8 & 4.3 \\
No & 96.8 & 95 & 97.5 & 97 & 95.3 & 95.8 \\
Number of respondents & 400 & 400 & 400 & 400 & 400 & 400 \\
\hline Did you seek treatment for pain during & \multicolumn{1}{c}{ urination } & & & & \\
Yes & 69.2 & 60 & 60 & 50 & 76.5 & 43.8 \\
No & 30.8 & 40 & 40 & 50 & 23.5 & 56.3 \\
Number of respondents & 13 & 20 & 10 & 12 & 17 & 16 \\
\hline Where did you seek treatment & & & & & & \\
Doctor & 66.7 & 66.7 & 16.7 & 66.7 & 83.3 & 57.1 \\
Dispenser/Paramedic & 0 & 0 & 0 & 0 & 10 & 0 \\
Traditional Practitioner & 33.3 & 58.3 & 83.3 & 33.3 & 10 & 28.6 \\
Self Medication & 11.1 & 16.7 & 16.7 & 0 & 20 & 0 \\
Others & 0 & 0 & 0 & 0 & 10 & 14.3 \\
Number of respondents & 9 & 12 & 6 & 6 & 10 & 7 \\
\hline
\end{tabular}




\begin{tabular}{|c|c|c|c|c|c|c|}
\hline & Faisalabad & Karachi & Lahore & Peshawar & Quetta & Rawalpindi \\
\hline \multicolumn{7}{|c|}{ Why did you not seek treatment } \\
\hline Not aware & 25 & 0 & 20 & 33.3 & 0 & 33.3 \\
\hline Facility not available & 0 & 14.3 & 0 & 16.7 & 0 & 0 \\
\hline Financial barriers & 25 & 25 & 0 & 33.3 & 25 & 11.1 \\
\hline Shyness & 25 & 0 & 25 & 33.3 & 25 & 11.1 \\
\hline Others & 50 & 71.4 & 80 & 33.3 & 33.3 & 66.7 \\
\hline No information & 0 & 12.5 & 25.0 & 0 & 25.0 & 0 \\
\hline Number of respondents & 4 & 7 & 5 & 6 & 3 & 9 \\
\hline \multicolumn{7}{|c|}{ What was the problem diagnosed as } \\
\hline Problem not diagnosed & 0 & 50 & 50 & 14.3 & 16.7 & 22.2 \\
\hline Gonorrhea (Sozaak) & 11.1 & 0 & 0 & 14.3 & 58.3 & 22.2 \\
\hline Hepatitis & 11.1 & 0 & 0 & 0 & 0 & 0 \\
\hline Others & 77.8 & 50 & 50 & 71.4 & 25 & 55.6 \\
\hline No Information & 11.1 & 8.3 & & & 20.0 & 25.0 \\
\hline Number of respondents & 9 & 12 & 6 & 7 & 12 & 9 \\
\hline \multicolumn{7}{|c|}{ How was the problem diagnosed } \\
\hline History/ sign symptoms & 62.5 & 66.7 & 50 & 50 & 55.6 & 50 \\
\hline Lab test & 37.5 & 33.3 & 25 & 50 & 33.3 & 33.3 \\
\hline Others & 0 & 0 & 25 & 0 & 11.1 & 16.7 \\
\hline Number of respondents & 8 & 6 & 4 & 8 & 9 & 6 \\
\hline \multicolumn{7}{|c|}{ Was your partner aware about your disease } \\
\hline Yes & 30 & 18.2 & 55.6 & 20 & 40 & 50 \\
\hline No & 70 & 81.8 & 44.4 & 80 & 60 & 50 \\
\hline Number of respondents & 10 & 11 & 9 & 5 & 10 & 10 \\
\hline \multicolumn{7}{|c|}{ What preventive measure did you use to prevent your partner } \\
\hline Nothing & 90.9 & 50 & 66.7 & 44.4 & 69.2 & 55.6 \\
\hline Avoid intercourse & 0 & 14.3 & 11.1 & 0 & 0 & 22.2 \\
\hline Used condom & 0 & 7.1 & 0 & 11.1 & 15.4 & 0 \\
\hline Never had sex & 9.1 & 7.1 & 11.1 & 11.1 & 7.7 & 11.1 \\
\hline Partner's treatment & 0 & 0 & 0 & 11.1 & 0 & 0 \\
\hline Others & 0 & 21.4 & 11.1 & 22.2 & 7.7 & 11.1 \\
\hline No Information & 15.4 & 30.0 & 10.0 & 16.7 & 31.6 & 47.1 \\
\hline Number of respondents & 11 & 14 & 9 & 9 & 13 & 9 \\
\hline
\end{tabular}


Table A1.12: Reason for using condom last time according to type of partner, by city $(n=130)$

\begin{tabular}{lrrrrrr}
\hline Partner & Faisalabad & Karachi & Lahore & Peshawar & Quetta & Rawalpindi \\
\hline Female (not a sex worker) - FOSW & & & & & & \\
Avoid pregnancy & 86.7 & 100.0 & 73.3 & 66.7 & 60.0 & 88.2 \\
Avoid giving a disease & 40.0 & 41.7 & 20.0 & 50.0 & 0.0 & 23.5 \\
Avoid getting a disease & 53.3 & 25.0 & 20.0 & 66.7 & 50.0 & 47.1 \\
Partner's wish & 6.7 & 0.0 & 6.7 & 0.0 & 0.0 & 0.0 \\
Other & 6.7 & 0.0 & 0.0 & 0.0 & 0.0 & 0.0 \\
(Number of respondents) & $\mathbf{( 1 5 )}$ & $\mathbf{( 1 2 )}$ & $\mathbf{( 1 5 )}$ & $\mathbf{( 6 )}$ & $\mathbf{( 1 0 )}$ & $\mathbf{( 1 7 )}$ \\
\hline Female sex worker - FSW & & & & & & \\
Avoid pregnancy & 75.0 & 37.5 & 25.0 & 25.0 & 0.0 & 37.5 \\
Avoid giving a disease & 25.0 & 25.0 & 25.0 & 25.0 & 11.1 & 12.5 \\
Avoid getting a disease & 100.0 & 37.5 & 62.5 & 50.0 & 88.9 & 62.5 \\
Partner's wish & 0.0 & 0.0 & 25.0 & 25.0 & 0.0 & 0.0 \\
Other & 0.0 & 0.0 & 0.0 & 0.0 & 0.0 & 12.5 \\
(Number of respondents) & $\mathbf{( 4 )}$ & $\mathbf{( 8 )}$ & $\mathbf{( 8 )}$ & $\mathbf{( 4 )}$ & $\mathbf{( 9 )}$ & $\mathbf{( 8 )}$ \\
\hline Male (not a sex worker) - MOSW & & & & & & \\
Avoid giving a disease & 0.0 & 100.0 & 0.0 & 0.0 & 0.0 & 0.0 \\
Avoid getting a disease & 100.0 & 0.0 & 0.0 & 0.0 & 0.0 & 100.0 \\
Other & 0.0 & 0.0 & 0.0 & 100.0 & 0.0 & 0.0 \\
(Number of respondents) & $\mathbf{( 1 )}$ & $\mathbf{( 1 )}$ & $\mathbf{( 0 )}$ & $\mathbf{( 1 )}$ & $\mathbf{( 0 )}$ & $\mathbf{( 1 )}$ \\
\hline Hijra & & & & & & \\
Avoid getting a disease & 100.0 & 0.0 & 0.0 & 0.0 & 0.0 & 0.0 \\
Other & 0.0 & $\mathbf{0}$ (N).0 & 0.0 & 0.0 & 0.0 & 100.0 \\
(Number of respondents) & $\mathbf{( 2 )}$ & $\mathbf{( 0 )}$ & $\mathbf{( 0 )}$ & $\mathbf{( 0 )}$ & $\mathbf{( 0 )}$ & $\mathbf{( 1 )}$ \\
\hline & & & & & &
\end{tabular}

Table A1.13: Consistency of condom use in last 12 months according to type of partner, by city $(\mathrm{n}=370)$ (percent)

\begin{tabular}{lrrrrrr}
\hline $\begin{array}{l}\text { Type of partner } / \\
\text { condom use }\end{array}$ & Faisalabad & Karachi & Lahore & Peshawar & Quetta & Rawalpindi \\
\hline $\begin{array}{l}\text { Female not sex worker (FOSW) } \\
\text { Every time }\end{array}$ & 6.0 & 7.1 & 10.0 & 18.8 & 16.1 & 14.7 \\
Often & 3.0 & 4.8 & 6.7 & 0.0 & 3.2 & 11.8 \\
Sometimes & 22.4 & 4.8 & 23.3 & 12.5 & 22.6 & 23.5 \\
Never & 68.7 & 83.3 & 60.0 & 68.8 & 58.1 & 50.0 \\
(Number of respondents) & $(\mathbf{6 7 )}$ & $\mathbf{( 4 2 )}$ & $\mathbf{( 3 0 )}$ & $\mathbf{( 1 6 )}$ & $\mathbf{( 3 1 )}$ & $\mathbf{( 3 4 )}$ \\
\hline Female sex worker (FSW) & & & & & & \\
Every time & 6.9 & 9.1 & 16.7 & 0.0 & 12.5 & 11.8 \\
Often & 6.9 & 12.1 & 4.2 & 0.0 & 0.0 & 17.6 \\
Sometimes & 20.7 & 0.0 & 20.8 & 33.3 & 20.8 & 11.8 \\
Never & 65.5 & 78.8 & 58.3 & 66.7 & 66.7 & 58.8 \\
(Number of respondents) & $\mathbf{( 2 9 )}$ & $\mathbf{( 3 3 )}$ & $\mathbf{( 2 4 )}$ & $\mathbf{( 1 2 )}$ & $\mathbf{( 2 4 )}$ & $\mathbf{( 1 7 )}$ \\
\hline Male sex worker (MSW) & & & & & & \\
Every time & 0.0 & 0.0 & 0.0 & 0.0 & 0.0 & 0.0 \\
Often & 0.0 & 0.0 & 0.0 & 0.0 & 0.0 & 0.0 \\
Sometimes & 0.0 & 0.0 & 0.0 & 0.0 & 0.0 & 0.0 \\
Never & 100.0 & 100.0 & 100.0 & 100.0 & 100.0 & 100.0 \\
(Number of respondents) & $\mathbf{( 2 )}$ & $\mathbf{( 1 2 )}$ & $\mathbf{( 1 )}$ & $\mathbf{( 3 )}$ & $\mathbf{( 1 0 )}$ & $\mathbf{( 4 )}$
\end{tabular}




\begin{tabular}{|c|c|c|c|c|c|c|}
\hline $\begin{array}{l}\text { Type of partner / } \\
\text { condom use }\end{array}$ & Faisalabad & Karachi & Lahore & Peshawar & Quetta & Rawalpindi \\
\hline \multicolumn{7}{|c|}{ Male not sex worker (MOSW) } \\
\hline Every time & 0.0 & 0.0 & 0.0 & 0.0 & 0.0 & 0.0 \\
\hline Often & 0.0 & 0.0 & 0.0 & 0.0 & 0.0 & 0.0 \\
\hline Sometimes & 11.1 & 0.0 & 0.0 & 0.0 & 6.3 & 11.1 \\
\hline 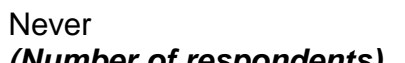 & 88.9 & 100.0 & 100.0 & 100.0 & 93.8 & 88.9 \\
\hline \multicolumn{7}{|l|}{ Hijra } \\
\hline Every time & 20.0 & 0.0 & 0.0 & 0.0 & 0.0 & 0.0 \\
\hline Often & 0.0 & 0.0 & 0.0 & 0.0 & 0.0 & 0.0 \\
\hline Sometimes & 0.0 & 0.0 & 0.0 & 0.0 & 0.0 & 100.0 \\
\hline Never & 80.0 & 100.0 & 100.0 & 100.0 & 100.0 & 0.0 \\
\hline (Number of respondents) & (5) & (7) & (2) & (1) & (1) & (1) \\
\hline
\end{tabular}

Table A1.14: STI prevalence, by city (by individual infected cases)

\begin{tabular}{lrrrrrrrrrrrrr}
\hline & \multicolumn{1}{c}{ Total } & \multicolumn{3}{c}{ Syphilis/TPHA } & \multicolumn{1}{c}{ HIV } & \multicolumn{3}{c}{ HSV 2 } & \multicolumn{2}{c}{ Chlamydia } & Gonorrhea & \multicolumn{3}{c}{ Overall } \\
\cline { 2 - 15 } & $\mathbf{N}$ & $\mathbf{N}$ & $\%$ & $\mathbf{N}$ & $\%$ & $\mathbf{N}$ & $\%$ & $\mathbf{N}$ & $\%$ & $\mathbf{N}$ & $\%$ & $\mathbf{N}$ & $\%$ \\
\hline Rawalpindi & $\mathbf{3 9 9}$ & 5 & 1.25 & 0 & 0.00 & 7 & 1.75 & 0 & 0.00 & 1 & 0.25 & $\mathbf{1 3}$ & $\mathbf{3 . 0 1}$ \\
Lahore & $\mathbf{3 9 2}$ & 4 & 1.02 & 0 & 0.00 & 17 & 4.34 & 0 & 0.00 & 0 & 0.00 & $\mathbf{2 1}$ & $\mathbf{5 . 1 0}$ \\
Faisalabad & $\mathbf{4 0 4}$ & 7 & 1.73 & 3 & 0.74 & 10 & 2.48 & 0 & 0.00 & 0 & 0.00 & $\mathbf{2 0}$ & $\mathbf{3 . 7 1}$ \\
Karachi & $\mathbf{4 0 0}$ & 15 & 3.75 & 0 & 0.00 & 24 & 6.00 & 0 & 0.00 & 1 & 0.25 & $\mathbf{4 0}$ & $\mathbf{8 . 5 0}$ \\
Quetta & $\mathbf{3 9 7}$ & 1 & 0.25 & 0 & 0.00 & 17 & 4.28 & 0 & 0.00 & 0 & 0.00 & $\mathbf{1 8}$ & $\mathbf{4 . 2 8}$ \\
Peshawar & $\mathbf{4 0 1}$ & 0 & 0.00 & 0 & 0.00 & 8 & 2.00 & 0 & 0.00 & 0 & 0.00 & $\mathbf{8}$ & $\mathbf{2 . 0 0}$ \\
Total & $\mathbf{2 , 3 9 3}$ & $\mathbf{3 2}$ & $\mathbf{1 . 3 4}$ & $\mathbf{3}$ & $\mathbf{0 . 1 3}$ & $\mathbf{8 3}$ & $\mathbf{3 . 4 7}$ & $\mathbf{0}$ & $\mathbf{0 . 0 0}$ & $\mathbf{2}$ & $\mathbf{0 . 0 8}$ & $\mathbf{1 2 0 ^ { 2 }}$ & $\mathbf{4 . 4 3}$ \\
\hline
\end{tabular}

${ }^{a}$ The total number of STIs diagnosed was 120 in 106 individuals, signifying that 14 individuals in the study were suffering from multiple STIs. 
Table A1.15: Sociodemographic characteristics of bridging and non-bridging population

\begin{tabular}{|c|c|c|c|c|c|c|c|c|c|c|}
\hline \multirow{3}{*}{ Characteristic } & \multicolumn{4}{|c|}{ Population } & \multicolumn{6}{|c|}{ Population (Cl 95\%) } \\
\hline & \multicolumn{2}{|c|}{ Bridging } & \multicolumn{2}{|c|}{ Non-bridging } & \multicolumn{3}{|c|}{ Bridging } & \multicolumn{3}{|c|}{ Non-bridging } \\
\hline & $\%$ & $\mathbf{N}$ & $\%$ & $\mathbf{N}$ & Mid & Lower & Upper & Mid & Lower & Upper \\
\hline \multicolumn{11}{|l|}{ Age } \\
\hline $16-20$ yrs & 26.7 & 55 & 18.5 & 405 & 26.7 & 21.1 & 33.1 & 18.5 & 16.9 & 20.1 \\
\hline $21-25$ yrs & 39.8 & 82 & 22.4 & 491 & 39.8 & 33.4 & 46.6 & 22.4 & 20.7 & 24.2 \\
\hline $26-30$ yrs & 19.4 & 40 & 17.4 & 382 & 19.4 & 14.6 & 25.4 & 17.4 & 15.9 & 19.1 \\
\hline $31-35$ yrs & 7.3 & 15 & 12.1 & 266 & 7.3 & 4.5 & 11.7 & 12.1 & 10.8 & 13.6 \\
\hline $36-40$ yrs & 4.9 & 10 & 13.8 & 303 & 4.9 & 2.7 & 8.7 & 13.8 & 12.4 & 15.3 \\
\hline $\begin{array}{l}41-45 \text { yrs } \\
\text { Total }\end{array}$ & $\begin{array}{r}1.9 \\
100.0 \\
\end{array}$ & $\begin{array}{r}4 \\
206 \\
\end{array}$ & $\begin{array}{r}15.8 \\
100.0 \\
\end{array}$ & $\begin{array}{r}347 \\
2,194 \\
\end{array}$ & 1.9 & 0.8 & 4.9 & 15.8 & 14.4 & 17.4 \\
\hline Marital status & & & & & Mid & Lower & Upper & Mid & Lower & Upper \\
\hline Ever married & 26.2 & 54 & 55.0 & 1207 & 26.2 & 20.68 & 32.6 & 55.0 & 52.9 & 57.1 \\
\hline $\begin{array}{l}\text { Never married } \\
\text { Total }\end{array}$ & $\begin{array}{r}73.8 \\
100.0 \\
\end{array}$ & $\begin{array}{l}152 \\
206 \\
\end{array}$ & $\begin{array}{r}45.0 \\
100.0 \\
\end{array}$ & $\begin{array}{r}987 \\
2,194 \\
\end{array}$ & 73.8 & 67.38 & 79.3 & 45.0 & 42.9 & 47.1 \\
\hline Education & & & & & Mid & Lower & Upper & Mid & Lower & Upper \\
\hline Illiterate & 17.5 & 36 & 14.6 & 321 & 17.5 & 12.9 & 23.2 & 14.6 & 13.2 & 16.2 \\
\hline Primary & 41.7 & 86 & 27.0 & 593 & 41.7 & 35.2 & 48.6 & 27.0 & 25.2 & 28.9 \\
\hline Secondary & 24.3 & 50 & 24.9 & 547 & 24.3 & 18.9 & 30.6 & 24.9 & 23.2 & 26.8 \\
\hline $\begin{array}{l}\text { Above secondary } \\
\text { Total }\end{array}$ & $\begin{array}{r}16.5 \\
100.0 \\
\end{array}$ & $\begin{array}{r}34 \\
206 \\
\end{array}$ & $\begin{array}{r}33.4 \\
100.0 \\
\end{array}$ & $\begin{array}{r}733 \\
2,194 \\
\end{array}$ & 16.5 & 12.1 & 22.2 & 33.4 & 31.5 & 35.4 \\
\hline Employment & & & & & Mid & Lower & Upper & Mid & Lower & Upper \\
\hline Unemployed & 5.3 & 11 & 2.3 & 50 & 5.3 & 3.0 & 9.3 & 2.3 & 1.7 & 3.0 \\
\hline Student & 13.6 & 28 & 4.6 & 101 & 13.6 & 9.6 & 18.9 & 4.6 & 3.8 & 5.6 \\
\hline Government service & 4.9 & 10 & 21.6 & 473 & 4.9 & 2.7 & 8.7 & 21.6 & 19.9 & 23.3 \\
\hline Private service & 40.3 & 83 & 31.8 & 698 & 40.3 & 33.8 & 47.1 & 31.8 & 29.9 & 33.8 \\
\hline Self employed & 27.2 & 56 & 31.4 & 689 & 27.2 & 21.6 & 33.6 & 31.4 & 29.5 & 33.4 \\
\hline $\begin{array}{l}\text { Other } \\
\text { Total }\end{array}$ & $\begin{array}{r}8.7 \\
100.0 \\
\end{array}$ & $\begin{array}{r}18 \\
206 \\
\end{array}$ & $\begin{array}{r}8.4 \\
100.0 \\
\end{array}$ & $\begin{array}{r}183 \\
2,194 \\
\end{array}$ & 8.7 & 5.6 & 13.4 & 8.4 & 7.3 & 9.6 \\
\hline Monthly earnings & & & & & Mid & Lower & Upper & Mid & Lower & Upper \\
\hline Student/unemployed & 19.2 & 39.552 & 8.4 & 185 & 19.2 & 14.6 & 25.4 & 8.4 & 7.3 & 9.7 \\
\hline Up to Rs. 5,000 & 40.9 & 84.254 & 32.1 & 704 & 40.9 & 34.3 & 47.6 & 32.1 & 30.2 & 34.1 \\
\hline Rs. $5,001-15,000$ & 35.0 & 72 & 48.6 & 1066 & 35.0 & 28.8 & 41.7 & 48.6 & 46.5 & 50.7 \\
\hline $\begin{array}{l}\text { More than Rs. } 15,000 \\
\text { Total }\end{array}$ & $\begin{array}{r}4.9 \\
100.0\end{array}$ & $\begin{array}{r}10 \\
206\end{array}$ & $\begin{array}{r}10.9 \\
100.0\end{array}$ & $\begin{array}{r}240 \\
2,194\end{array}$ & 4.9 & 2.7 & 8.7 & 10.9 & 9.7 & 12.3 \\
\hline
\end{tabular}


Figure A1.1: Condom use during last (non-marital) sexual intercourse, by age and city $(n=697)$

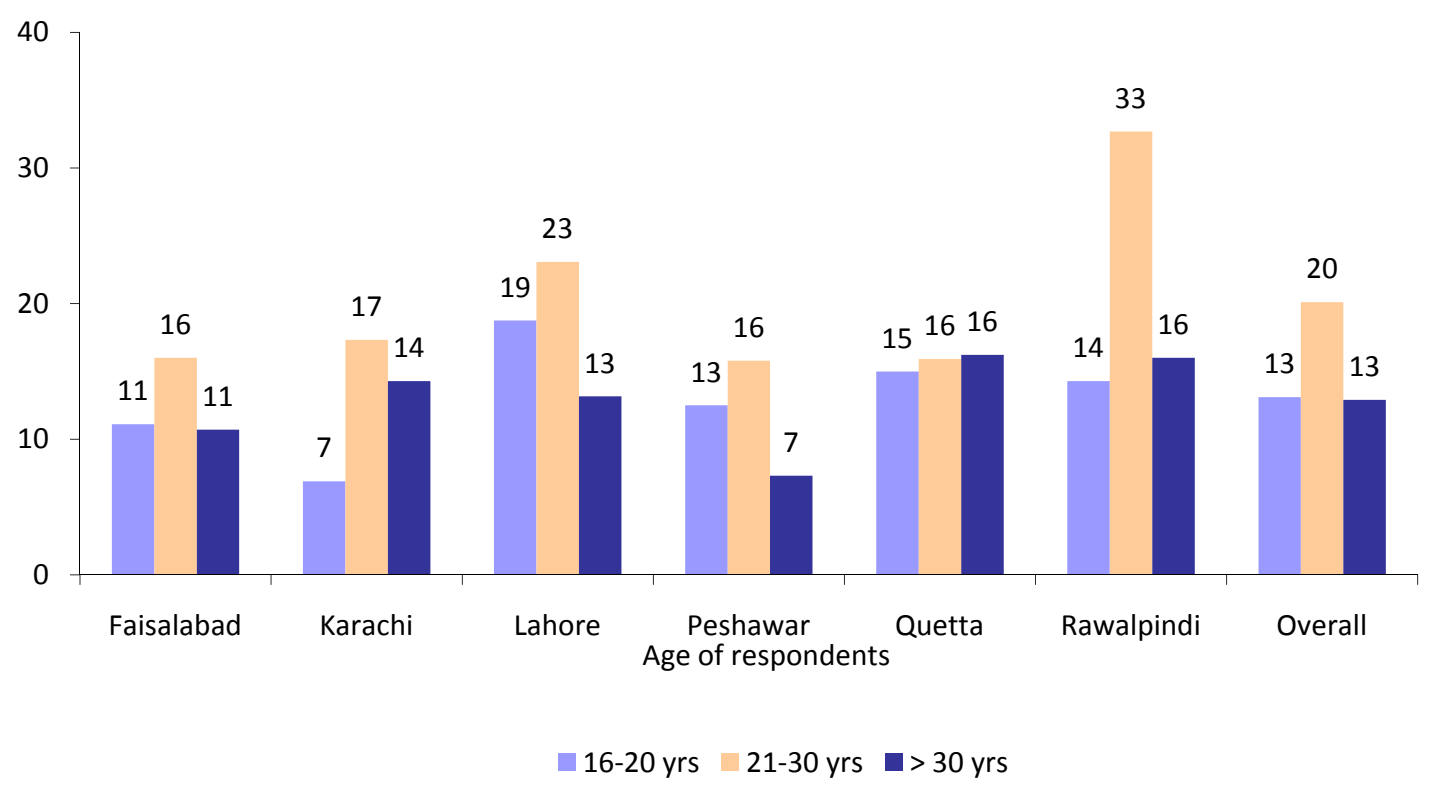

Figure A1.2: Condom use during last (non-marital) sexual intercourse, by educational level and city (percent) $(n=697)$

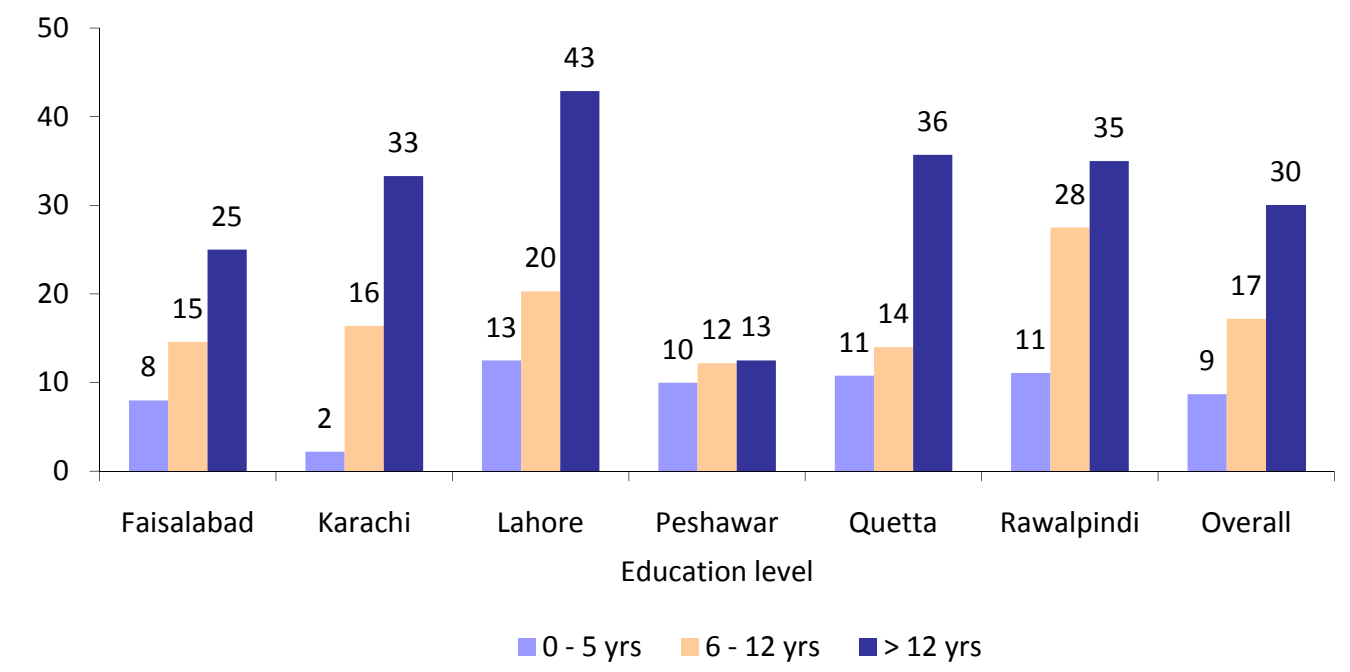


Figure A1.3: Condom use during last non-marital sexual intercourse, by marital status and city $(n=697)$

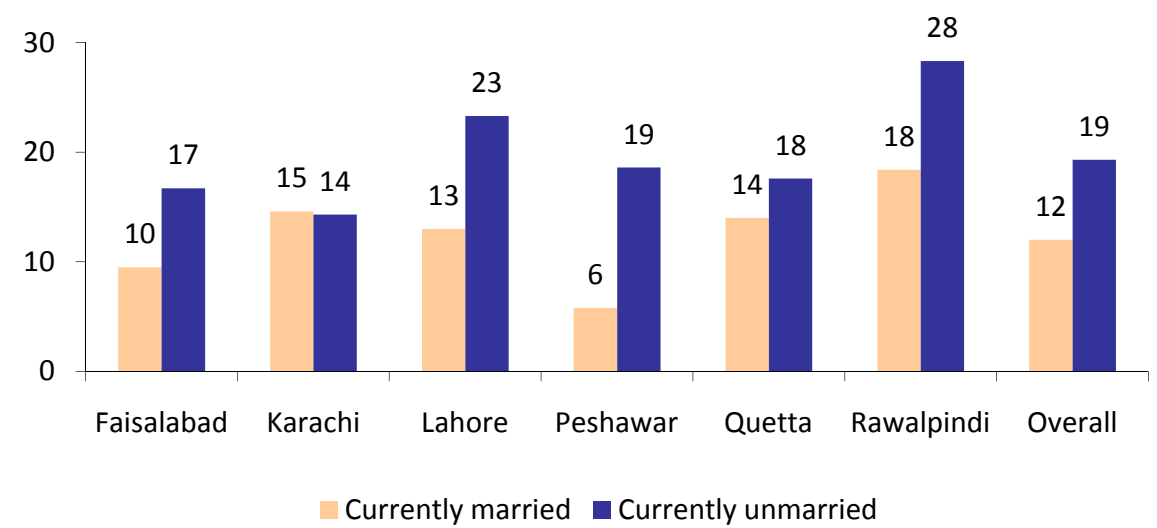

Figure A1.4: Condom use at last non-marital sexual intercourse, by migrant status and city (percent) $(n=697)$

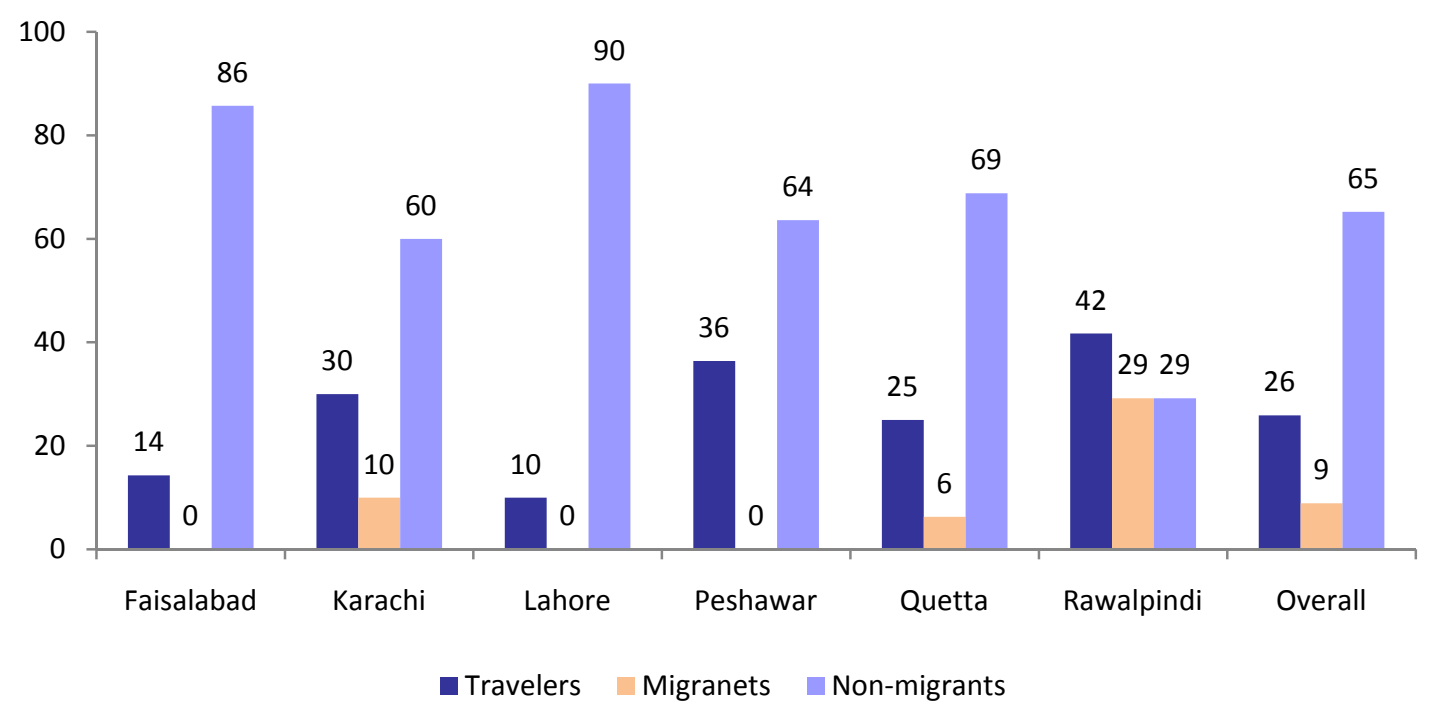





\section{APPENDIX 2: ETHICAL APPROVAL LETTERS}

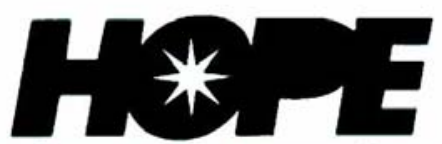

Health Oriented Preventive Education
5. AMR KHUSRO ROAD, QLOCK $7 / 8$ OVERSEAS CO-OPERATME
HOUSING SOCIETY KARACHI-PAKISTAN E-mali: ogboat@gerrs net
Pn: 4539393

To.

Dated: - 22nd May’07

Dr.Ali Mohammed Mir,

Director Programmes

Population Council

\# 7. Street 62,F-6/3,

Islamabad.

Subject: -

Ethical Approval for Study of Sexually Transmitted Infections

Survey of the Bridging Population

Dear Dr.Ali: Mohammed Mir,

This is with reference to your letter dated $9^{\text {th }}$ May 07 regarding ERB approval for your project titled "Study of Sexually Transmitted Infections,Survey of Bridging Population" The Ethical Review Board of HOPE, has given approval for the project for a period of one year from today's date $22^{\text {nd }}$ May' 07 .

Any change in the proposal should be notified to the committee in four weeks.

Best Regards,

Yours truly,

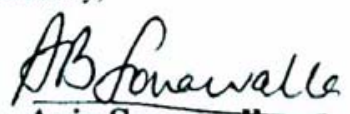

Dr. Aziz Sonawalla

Human Research Review Committee

HOPE 


\title{
QP Population Council
}

\author{
Institutional Review Board \\ Population Council \\ 1230 York Avenue \\ New York, New York 10021
}

APPROVAL OF PROTOCOL

\author{
DATE: $\quad$ March 7, 2007 \\ TO: $\quad$ Ali Mohammad Mir, Principal Investigator \\ FROM: $\quad$ Sheldon J. Segal, Chairman \\ Institutional Review Board \\ RE: $\quad$ Approval of Protocol No. 404: National study of STIs among urban men in Pakistan
}

The Institutional Review Board (IRB) on human research of the Population Council has approved the above request to involve humans as research subjects.

APPROVAL DATE OF PROTOCOL:

FEBRUARY 14, 2007

ADVERSE REACTIONS/COMPLICATIONS: All serious and/or unexpected side effects must be reported immediately by fax to the Population Council's SAE Desk (SAE Desk Fax No.: 212-3278673), which will notify the IRB of the Population Council.

MODIFICATIONS: All protocol changes involving subjects must have prior IRB approval.

If this project is to continue, it must be renewed as specified by the IRB. THE EXPIRATION DATE FOR THIS PROJECT IS FEBRUARY 14, 2008. This renewal application consists of a brief status report summarizing the results obtained during the past period and a short statement of the research plan for the coming year.

If you have any questions, please contact Jean Schweis at telephone No. [212] 327-8719 or FAX No. [212] 327-7678.

cc: IRB Records and Reports File for Protocol 404 J. van Dam, Study Coordinator 
LONDON SCHOOL OF HYGIENE

\& TROPICAL MEDICINE

ETHICS COMMITTEE

\section{APPROVAL FORM}

Rade 24107107

Application number:

5118

Name of Principal Investigator

Dr Sarah Hawkes

Department

Infectious and Tropical Diseases

Head of Department

Hazel Dockrell

Title: Pakistan National Study of Reproductive Tract Infections, Bridging Population Study

Approval of this study is granted by the Committee.

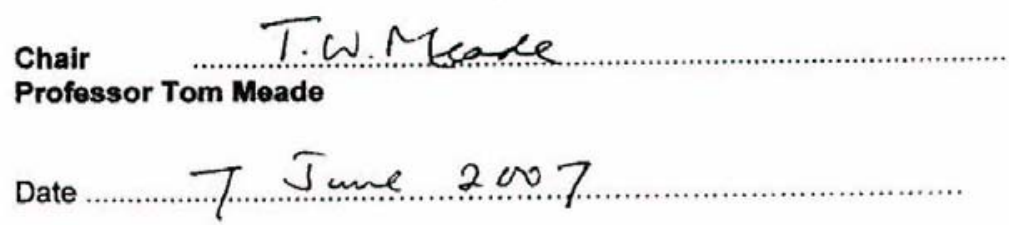

Approval is dependent on local ethical approval having been received.

Any subsequent changes to the consent form must be re-submitted to the Committee. 



\section{APPENDIX 3. QUALITATIVE RESEARCH}

\section{Objectives}

In order to assess men's perceptions regarding sexually transmitted infections, as well as to obtain a better understanding of the prevailing sexual behaviors and norms, 64 focus group discussions were conducted prior to the quantitative survey. The findings have helped in the development of the survey questionnaire, and have also helped in better understanding the findings we have obtained quantitatively.

The research focused on the general perceptions of the target groups in the context of sexually transmitted infections, and on their sexual practices. The main objectives are provided below.

Objective 1. To assess the level of awareness among the target groups regarding STIs including their symptoms, mode of transmission and prevention.

Objective 2. To identify the various myths and misconceptions among the target populations regarding STIs and HIV/AIDS.

Objective 3. To understand the sexual behavior of the target population and the various ways of availing and accessing sexual services.

\section{Methodology}

The primary tool used for data collection was focus group discussions in four selected urban centers, one in each province. The age bracket of FGD participants was predetermined keeping in mind the active sexual age span of males of 16 to 45 years of age. Four categories of participants were selected in each city in order to understand the focus population, and also to conduct a comparative analysis between various categories of men. The four selected groups for each city were:

- Married migrants (MM)

- Unmarried migrants (UM) 
- Married non-migrants (MNM)

- Unmarried non-migrants (UNM)

A total of 452 male respondents participated in the 64 FGDs, with each FGD comprising a minimum of five and a maximum of ten participants. During the initial phase of the broader study, 16 focus group discussions were conducted in the four selected cities according to the four categories of respondents. These pilot focus group discussions were treated as a formative research for refining the survey tools and identification of appropriate locations within the respective cities. Taking into consideration the significant findings from the earlier FGD pilots, an additional 48 FGDs were conducted to better understand and explore the knowledge, attitudes and practices of the target population.

As already described in the methodology section, key informants identified the FGD participants in each city. It was important to have an insight into the appropriate localities and access to the target population, and obtain the consensus of the right target group for participation in the discussions. A consent form was circulated prior to all discussions, stating the nature of the discussion, its purpose and adherence to principles of confidentiality.

For a balanced representation of participants, males from low income to middle income were selected for the FGDs, with the presumption that men from these income groups would be more vulnerable than higher income groups. Occupation groups varied from daily laborers, property dealers, businessmen, shopkeepers and university employees, while a significant proportion of unmarried males were students or currently searching for employment. Similarly, education levels of the respondents also differed, with lower income groups having lower education levels as compared to those in the service and private sectors.

A team of social science interviewers - experienced facilitators and note takers fluent in the local dialects - received a four-day training, as already described, and then conducted the FGDs with the help of a guideline (see appendix 7).

The data analysis of the FGDs was conducted manually by organizing the interview transcripts into separate categories of each study target group from all four provinces, as well as further analyzing cross-provincial differentials wherever deemed appropriate. The comparative analysis between migrant and non-migrant groups of 
participants was also conducted manually, according to the information presented, through an analysis matrix developed for the purpose.

The sample size of 64 focus group discussions is quite large; therefore, a matrix of key indicators was developed, which helped in data compilation and analysis of the information.

Some key socioeconomic indicators were also selected for collection of background information of each FGD participant. These data were tabulated, and frequencies have been used in understanding the socioeconomic profile of the group members according to the various group categories and regions.

The topic of sexually transmitted infections is a sensitive one, especially in the Pakistani sociocultural context, where there is a general perception of stigma and discrimination attached with any topic pertaining to human sexual behavior and practices. Thus, it was not an easy task to facilitate discussions on STIs and sexual behavior. There were many instances when the moderators had to stay neutral and objective during the discussions. Male respondents also tended to either underplay or overplay their sexual experiences in group settings, which could have direct implications for the accuracy of the information, which had to be determined by the group moderator.

It should also be mentioned that these FGDs were first conducted in local dialects, then translated into Urdu and finally translated into English. Unfortunately, many local nuances were lost during the translations; however, efforts were to retain as much of the local flavor of the discussions as possible.

\section{Summary of results}

The information obtained from the FGDs is indicative of the fact that there is a need to address the issues of STIs among the male population residing in cities of Pakistan, with emphasis on migrant men. However, it is also apparent that not only migrant men but also the general population of men indulge in multiple sexual partners and activities. Therefore, interventions regarding STIs should cover the general population, with particular services for migrant males in localities of major urban centers with large migrant populations. 
The following is a summary of the major findings from the focus group discussions.

- Provincial differentials are not prominent, with attitudes and practices concerning STIs being similar across all four provinces.

- There is a growing awareness among the men regarding STIs; however, their knowledge is limited to basic information attained through television, friends, and other media sources.

- Myths and misconceptions concerning STIs, especially HIV/AIDS, are widespread; incomplete information is further distorted and misguides people.

- The concept of masculinity is associated with sexual skills and performance rather than traits such as strength, power or piousness. Men also felt that masculinity was linked to power and strength to produce children.

- Sexual activities are common, and both paid and unpaid non-marital sex is taking place. Brothel-based sex is now being replaced by home-based sex mediated through mobile phones. Unpaid sex is reportedly taking place with friends who are either neighbors, colleagues, or class fellows; however, to some degree, a transaction 'in-kind' does take place. Multiple sexual partners are used according to what the client can afford.

- Heterosexual sex is preferred; however, in some regions, like the North West Frontier Province, homosexuality is also socially accepted among the male groups. Sexual contact with transsexuals (hijras) is also practiced, but is not very popular as it is perceived as dangerous and "low class."

- Sex workers were mostly availed and personal one-to-one relationships are very rare. Men buy sex according to their financial status; they also indulge in group sexual activities to save money and because they find group sex more entertaining.

- A significant number of men were aware of condom use for prevention against disease and also mentioned that many sex workers now carried condoms for their clients. But its usage was still low and men preferred sex without condoms. The availability of condoms at the time of sex was another issue mentioned by some respondents as urgency at that particular point in time prevented them from using a condom.

- Masturbation is very common, but is associated with guilt and misconceptions (e.g., negative impact on male potency).

- Due to lack of awareness and hesitancy in disclosing a sexually related problem, males suffering from symptoms of STIs consulted traditional healers. 
Other reasons for consulting traditional healers included social stigma, cost factors and the nonjudgmental attitude of this service provider.

- Although media was the main source of information for the majority of men, it is perceived as having a negative impact, exposing people to various sexual provocations. Internet was another negatively perceived source of spreading sexual promiscuousness among the youth.

- Injecting drug use is not very common among the studied communities; however, there is a high awareness level among the men regarding the use of recycled syringes - there was a general agreement that injecting drug users share needles.

- Sex is not commonly sold or bought by IDUs, and men are not keen to buy sex from drug abusers.

- There is awareness among the men regarding STI transmission through blood transfusion, tattooing, and sharing of blades and syringes. Blood donations and tattooing are not very popular and very few men practice these.

\section{Research findings}

The information obtained through the FGDs indicates certain similarities across the four provinces, with no major differentials among migrant and non-migrant male participants. There are differences between married and unmarried respondents' behavior and attitudes toward STI issues, attitudes and practices related to sexual behavior. However, certain levels of preconceived notions are associated with certain ethnic groups. For example, participants in all four cities felt that homosexuality was more common in the North West Frontier Province, and that Baloch and Pathan men were more promiscuous as compared to Punjabi and Sindhi males.

The findings of this study have been divided into four main sections: 1) background characteristics of the respondents; 2) awareness regarding STIs; 3) sexual behavior; and prevention, awareness, and attitudes concerning sexual practices.

\section{Background characteristics of respondents}

The characteristics of the focus group participants are shown in table A2.1.

Age distribution for both of the unmarried groups ranged from 18 to 25 years, while for the married groups it was from 25 to 45 years. 
It was generally observed that migrant men lived in specific localities in the cities, sharing residential quarters with other migrant males in bachelor quarters with as many as 7-8 people per room. Most non-migrant men lived with their families, including those who were unmarried. According to the groups of non-migrants in all the four study cities, a joint family system was more cost effective. In addition, certain cultural practices also prevented them from leaving their extended families and living as a nuclear unit in the same city. This is important especially in the context of finding out if any group sexual activities are occurring.

The highest percentage of monthly income was below Rs. 10,000 (38 percent), followed by Rs. 10,000 to 15,000 (30 percent). However, a noticeable percentage of the FGD participants (12 percent) claimed to have a monthly income of more than Rs. 20,000, especially those who had their own private businesses.

Education levels were significantly high in the sample, with almost 50 percent having a college or university-level education. Only 7 percent of the participants had no education, with the highest non-literates in Quetta and Peshawar. There was variation in the occupation category, whereby many participants ( 24 percent) were students, and half were indulging in private-sector occupations, like marketing, property dealing, shopkeepers and employment in private organizations. Participants in public-sector employment were 11 percent overall, with the highest percentage among married nonmigrant males (57 percent), which among the provinces was highest in Quetta and Karachi (25 and 22 percent, respectively).

More than half of the participants were young males (between 20 and 30 years of age), while percentages declined as the age groups of the participants went up. Only 11 percent were less than 20 years old.

\section{Leisure time}

It was important to know how the respondents spent their free time after working hours for entertainment and relaxation, on the assumption that this would probably be related to their sexual behavior. This part of the discussion was held at the beginning of each FGD and served as an icebreaker. 
Table A2.1: Background characteristics of participants (percent)

\begin{tabular}{|c|c|c|c|c|c|c|c|c|c|c|c|c|c|c|c|c|c|}
\hline \multirow[b]{2}{*}{ Indicator } & \multicolumn{4}{|c|}{ Quetta } & \multicolumn{4}{|c|}{ Peshawar } & \multicolumn{4}{|c|}{ Faisalabad } & \multicolumn{4}{|c|}{ Karachi } & \multirow[b]{2}{*}{ Overall } \\
\hline & UNM & MNM & UM & MM & UNM & MNM & UM & MM & UNM & MNM & UM & MM & UNM & MNM & UM & MM & \\
\hline Nuclear/single & 0.0 & 47.8 & 56.5 & 28.5 & 0.0 & 14.2 & 16.6 & 45.0 & 0.0 & 15.0 & 57.8 & 20.0 & 0.0 & 52.1 & 76.1 & 66.6 & 36.6 \\
\hline Joint/shared & 100.0 & 52.1 & 43.4 & 71.4 & 100.0 & 85.7 & 83.3 & 55.0 & 100.0 & 85.0 & 36.8 & 80.0 & 100.0 & 47.8 & 23.8 & 33.3 & 63.4 \\
\hline \multicolumn{18}{|l|}{ Income } \\
\hline , Rs. 10,000 & 16.6 & 39.1 & 43.4 & 38.0 & 31.8 & 68.1 & 5.5 & 45.0 & 15.3 & 30.0 & 21.0 & 15.0 & 18.1 & 69.5 & 61.9 & 71.4 & 38.0 \\
\hline $10,000-15,000$ & 66.6 & 34.7 & 39.1 & 28.5 & 54.5 & 14.2 & 33.3 & 45.0 & 15.3 & 30.0 & 21.0 & 25.0 & 4.5 & 26.0 & 9.5 & 19.0 & 29.9 \\
\hline $16,000-20,000$ & 16.6 & 13.0 & 13.0 & 14.2 & 0.0 & 4.7 & 5.5 & 5.0 & 7.6 & 0.0 & 5.2 & 0.0 & 0.0 & 4.3 & 0.0 & 0.0 & 5.7 \\
\hline , Rs. 20,000 & 0.0 & 0.0 & 4.3 & 19.0 & 0.0 & 9.5 & 15.7 & 5.0 & 61.5 & 40.0 & 10.5 & 60.0 & 4.5 & 0.0 & 0.0 & 9.5 & 11.7 \\
\hline No response & 0.0 & 0.0 & 0.0 & 0.0 & 13.6 & 0.0 & 0.0 & 0.0 & 0.0 & 0.0 & 36.8 & 0.0 & 59.0 & 0.0 & 28.5 & 0.0 & 11.1 \\
\hline \multicolumn{18}{|l|}{ Education } \\
\hline Illiterate & 0.0 & 21.7 & 21.7 & & 0.0 & 23.8 & 0.0 & 10.0 & 0.0 & 0.0 & 0.0 & 0.0 & 0.0 & 4.3 & 19.0 & 0.0 & 6.6 \\
\hline Primary & 0.0 & 13.0 & 8.6 & $\mathrm{NR}^{*}$ & 4.5 & 14.2 & 0.0 & 5.0 & 0.0 & 5.0 & 0.0 & 0.0 & 0.0 & 13.0 & 38.0 & 19.0 & 7.8 \\
\hline Secondary & 20.8 & 13.0 & 26.0 & & 13.6 & 0.0 & 0.0 & 5.0 & 0.0 & 5.0 & 0.0 & 10.0 & 4.5 & 30.4 & 4.7 & 38.0 & 11.4 \\
\hline Matric & 37.5 & 26.0 & 8.6 & & 18.1 & 14.2 & 5.5 & 20.0 & 30.7 & 40.0 & 10.5 & 15.0 & 18.1 & 26.0 & 0.0 & 28.5 & 18.7 \\
\hline College & 41.6 & 21.7 & 34.7 & & 40.9 & 28.5 & 44.4 & 25.0 & 53.8 & 50.0 & 26.3 & 60.0 & 72.7 & 13.0 & 38.0 & 14.2 & 34.7 \\
\hline University & 0.0 & 4.3 & 0.0 & & 22.7 & 19.0 & 50.0 & 30.0 & 15.3 & 0.0 & 57.8 & 25.0 & 4.5 & 13.0 & 0.0 & 0.0 & 14.1 \\
\hline \multicolumn{18}{|l|}{ Employment } \\
\hline Students & 70.8 & 0.0 & 39.1 & 9.5 & 13.6 & 0.0 & 44.4 & 15.0 & 30.7 & 0.0 & 36.8 & 0.0 & 81.8 & 0.0 & 28.5 & 4.7 & 23.5 \\
\hline Unemployed & 0.0 & 26.0 & 0.0 & 0.0 & 0.0 & 0.0 & 0.0 & 0.0 & 23.0 & 5.0 & 0.0 & 0.0 & 0.0 & 0.0 & 0.0 & 0.0 & 3.0 \\
\hline Private sector & 29.1 & 34.7 & 21.7 & 52.3 & 72.7 & 33.3 & 55.5 & 75.0 & 46.1 & 90.0 & 47.3 & 70.0 & 18.1 & 65.2 & 61.9 & 38.0 & 50.1 \\
\hline Public sector & 0.0 & 25.2 & 0.0 & 4.7 & 0.0 & 57.1 & 0.0 & 5.0 & 0.0 & 0.0 & 10.5 & 30.0 & 0.0 & 21.7 & 0.0 & 0.0 & 10.8 \\
\hline Daily wage earner & 0.0 & 13.0 & 39.1 & 33.3 & 13.6 & 9.5 & 0.0 & 5.0 & 0.0 & 5.0 & 0.0 & 0.0 & 0.0 & 8.6 & 9.5 & 52.3 & 11.4 \\
\hline \multicolumn{18}{|l|}{ Age } \\
\hline Less than 20 & 41.6 & 0.0 & 43.4 & & 18.1 & 0.0 & 11.1 & 0.0 & 0.0 & 0.0 & 5.2 & 0.0 & 27.2 & 0.0 & 14.2 & 0.0 & 10.8 \\
\hline $20-25$ yrs & 50.0 & 30.4 & 39.1 & & 36.3 & 4.7 & 55.5 & 25.0 & 76.9 & 10.0 & 68.4 & 5.0 & 50.0 & 8.6 & 71.4 & 9.5 & 32.6 \\
\hline $26-30$ yrs & 0.0 & 17.3 & 17.3 & $N R^{*}$ & 40.9 & 19.0 & 33.3 & 40.0 & 23.0 & 30.0 & 21.0 & 20.0 & 18.1 & 13.0 & 0.0 & 23.8 & 22.6 \\
\hline $31-35$ yrs & 0.0 & 17.3 & 0.0 & & 4.5 & 23.8 & 0.0 & 30.0 & 0.0 & 15.0 & 0.0 & 15.0 & 4.5 & 17.3 & 14.2 & 28.5 & 13.2 \\
\hline $36-40$ yrs & 0.0 & 17.3 & 0.0 & & 0.0 & 19.0 & 0.0 & 5.0 & 0.0 & 35.0 & 0.0 & 40.0 & 0.0 & 30.4 & 0.0 & 9.5 & 9.9 \\
\hline $41-45$ yrs & 0.0 & 17.3 & 0.0 & & 0.0 & 33.3 & 0.0 & 0.0 & 0.0 & 10.0 & 0.0 & 20.0 & 0.0 & 30.4 & 0.0 & 28.5 & 9.0 \\
\hline $\begin{array}{l}\text { (Number of } \\
\text { participants) }\end{array}$ & (24) & (23) & (23) & (21) & (22) & (21) & (18) & (20) & (13) & (20) & (19) & (20) & (22) & (23) & (21) & (21) & (331) \\
\hline
\end{tabular}

MM = married migrant; UM = unmarried migrant; $\mathbf{M N M}=$ Married non-migrant; UNM = Unmarried non-migrant

${ }^{*}$ No response 
Most of the participants felt that leisure time was spent according to an individual's personal priorities. Regardless of their migrant/non-migrant status or marital status, men preferred spending time with their friends and doing joint activities, like playing cards or watching a movie.

"It depends on the mental approach; if a person is educated he spends his time studying. There are different categories of people - some spend time in gossip (gup shup), some play and some spend their time without any activity. Mostly my class fellows remain without any activity. Some are involved in gossip or in smoking, while others remain busy with their work.

Unmarried migrants, Karachi

Men also indulged in taking drugs or sexual activities outside of their homes during their free time. However, it depended upon the financial and social status of the individual, as well as the social environment of the locality. According to most participants, men were influenced by their friends to indulge in drugs or visit sex workers.

"Drug abuse is very common especially charas. Even the children use it so often. Most of the people who have nothing to do will be drug addicts or thieves because their main problem is joblessness. Those who are completely free also indulge in sex. They don't go to the mosque or keep any decent hobbies."

Married migrants, Peshawar

Using the Internet was also mentioned among the unmarried younger men, but only among those who had higher education, especially college and university level. Otherwise, it was not considered a very common leisure activity.

"I spend my time by browsing the Internet or reading the newspaper. Mostly people go for outings with their friend in their leisure time, do chatting on Internet and see TV."

Unmarried migrant, Karachi

Unmarried migrants in all of the cities also reported that they spent their free time on the streets, mostly with friends.

"Young boys just hang around on the streets, looking at girls, joking and playing with one another. 'Hanging around' is quite entertaining and relaxing for young boys as it gives them a chance to spend time with their friends."

Unmarried non-migrants, Faisalabad 
In some cases, it was obvious that migrant males spent their free time doing household chores rather than recreational activities, because they lived alone or in bachelors' quarters.

"During my free time, I do cooking and cleaning, because I don't get time during working hours. Generally, there is not enough free time for entertainment or relaxation activities."

Married migrants, Karachi

\section{Awareness regarding STls}

Sexually transmitted infections are a taboo subject, and men - young and old, married and unmarried - still tend to feel shy about acquiring information on STIs. During the FGDs, it was apparent that the most commonly known STI was HIV/AIDS, due to the media campaign associated with it. HIV/AIDS was invariably the first STI mentioned in all groups; this information came through television and newspapers. These findings agree with those from the quantitative data.

Although there was no difference in the responses of migrant and non-migrant groups, unmarried men had less information related to STIs and HIV/AIDS as compared to the married men. The probable reason for the married men having more information is the access and social legitimacy regarding information related to sex for those who are married.

Although the majority of the participants were aware of the term HIV/AIDS, most did not have a true understanding of the disease. Similarly, in the case of hepatitis, respondents were not sure about the type of infection. Many participants confused hepatitis A, B and C. The level of ignorance can be seen by a remark of one of the Karachi FGD participants:

"Besides hepatitis and AIDS, HIV is also very common and is transferred like TB."

Un-married migrant, Karachi

While HIV/AIDS and hepatitis were discussed in every group, only two participants actually described the symptoms of an AIDS infection. With the exception of these two participants, none of the other participants were aware of any symptoms of AIDS. One of the two who knew about AIDs symptoms said: 
"Its [AIDS] symptoms include physical weakness. The protective cells die and no cells are produced any longer resulting in a dysfunctional immune system. The body does not have any resistance any longer and the patient dies."

Married non-migrant, Karachi

Similarly, not a single group member discussed the symptoms of hepatitis. However, due to the high prevalence of hepatitis in the country, all men were very conscious of the infection, and many agreed that it had almost reached epidemic levels. The electronic and print media are very active in the providing public service messages concerning hepatitis.

"There is no AIDS patient here, but many people have hepatitis, which is also fatal. At least one person in every family has a hepatitis patient."

Married non-migrant, Quetta

Participants also mentioned other STIs, like syphilis and gonorrhea, using terms, such as galyan, ahtalum and sozak. Some of these are, indeed, local terms for gonorrhea and syphilis; however, it was also obvious through the information given in many groups that misconceptions were common and men were not clear about various diseases and their mode of transmission.

"Blood cancer and HIV/AIDS are the only two STIs I know about."

Unmarried migrant, Faisalabad

Several STIs were referred to by symptoms like pus and blood from the genitals, drops of seminal fluid before or after urine, genital warts and pimples, but there was no label attributed to these symptoms.

Several participants also specifically mentioned tuberculosis as another STI, while hepatitis B and C, typhoid, piles, diabetes and diarrhea were also termed as sexually transmitted infections. Incomplete information based on limited knowledge was commonly noticed among the participants.

"It is transferred by touching a patient with hepatitis C. There is a chance of getting infected if a person doesn't wash hands after touching the hepatitis $C$ patient. It is also transferred by sharing meals or by using reused syringes."

Married non-migrant, Karachi 


\section{Sources of knowledge}

Media was the most common source of knowledge regarding STIs among FGD participants. Most participants in all categories and regions said that television and newspapers were their primary sources of knowledge. HIV/AIDS and hepatitis messages through television were widely quoted.

"I got to know about HIV/AIDS through the television advertisement. In that message, they mention the various modes of transmission and how one should limit himself to his wife for prevention."

Married migrant, Faisalabad

Besides the media, other sources of information were mostly friends and health care providers. In Pakistan, the media has to use an indirect approach for transferring reproductive health and sexuality related information due to strict censorship policies; consequently, some of the information acquired by the recipient is vague and not properly comprehended. Similarly, friends and health care providers also fail to provide accurate knowledge resulting in increased misconceptions.

"I got my information from the doctor. If a person or a relative is suffering from this disease, and his relatives go to see him and are not careful, they can get infected. The doctor said to stay away from such patients."

Married migrant, Karachi

A few participants - mostly among the educated, unmarried participants - also mentioned the Internet as a source of information. However, a significant number of participants felt that the Internet was generally used for viewing pornographic material rather than for educational purposes. Several young males said that the Internet was a popular means of watching "blue movies" and other such pornographic material. But the use of the Internet was limited to a certain social strata and was not common in lowincome areas.

"In our locality even youngsters do not use the Internet that frequently. Only some educated youth use computers, otherwise we are not very familiar with it."

Married non-migrant, Quetta

Depending on the social strata of the person, according to some of the participants, Internet services could be acquired both at home (in case of those who were well-off), or in internet cafes in most localities of the cities. Young boys who generally could not afford internet services at their homes went to internet cafes with groups of friends. 
"With one computer card, young boys are able to watch many movies and visit various pornographic sites in the internet cafes. These cafes are quite common in most localities of the city and the owners are usually quite cooperative in context of guiding youngsters about their desired sites (mostly pornographic material)."

Unmarried migrant, Faisalabad

Another source of STI information was 'wall chalking' (writing on walls), usually used by hakeems and pirs as advertisements for their services. Because hakeems and pirs were also important sources for treatment among many men for STIs, wall chalking provided useful information about the mode of treatment for STIs.

"Wall chalking is quite visible on the walls of all the main roads of the city. They are direct and easy to understand as compared to information provided in the newspapers of shown on television. Therefore, many men prefer going to hakeems for such problems as STIs."

Unmarried non-migrant, Peshawar

During two FGDs (one in Karachi and one in Faisalabad) a few men also mentioned NGOs as another source of information for such diseases as AIDS and hepatitis. Probably, some NGOs were engaged in local awareness raising and providing preventive services for HIV/ AIDS and other STIs.

"NGO's are working a lot on it [STIs]; due to this, the public get more awareness and help. Lots of programs are telecast. Medical agents and company campaigns by pharmaceutical companies are also giving information to people. For example the company I am working with provides us with information on such subjects."

Married migrant, Faisalabad

\section{Vulnerability to STIs}

In order to have a more in-depth perspective of the understanding of STIs within the target groups, the respondents were asked what kind of people were more vulnerable of getting infected with an STI. The majority of respondents said that people who did not have proper information and awareness, and those who indulged in promiscuous behavior, were more likely to get STIs. A significant number of men also felt that those who had drawn away from Islam and did not follow appropriate religious practices suffered from STIs. 
"Those men and women go through these problems who do not follow the correct principles of Islam and allow Satan to lead them. If everyone followed Islam in the right way, our society would be clean of all such menaces."

Married non-migrant, Quetta

"Men and women who sleep around and have sex with other people besides their spouses get such diseases as AIDS and syphilis. Indulging in illegitimate sex is a sin; therefore, God punishes such people in this way."

Married migrant, Peshawar

However, there were some participants with unbiased attitudes who said that anyone could get any disease, including STIs, and that sexual contact was not the only mode of transmission and STIs could be passed on through other means as well.

"HIV/AIDS or any other STI is not transmitted through sex only. They can be passed on through blood transfusion or using a used syringe. So we should not pass judgment on those who have such an infection."

Married non-migrant, Karachi

"It is all in the hands of God. He is the one who gives life to a dying person. We should never say that we got infection from that person."

Married migrant, Quetta

It was also a common perception among a large number of participants that sex workers suffered more from such ailments due to their professional activities.

"Women who are professional sex workers have STIs because they have sex with so many men and also do not use any precautions either. Due to them, these diseases are spread from one person to another."

Unmarried migrant, Faisalabad

\section{Stigma and discrimination}

Stigma and discrimination against STI patients was more prominent among the married men as compared to younger unmarried men. The apparent reason for a higher level of stigma and discrimination among the married men was less exposure and awareness as compared to the unmarried men, who were younger and had more knowledge about such issues due to higher educational level, access to Internet and other electronic media, and more socializing and communication opportunities.

Besides marital status and age, education level of the participants also contributed in opinion formation against STI-infected people. The higher the education level of the 
participant, the less judgmental he was about those with STIs. However, almost everyone agreed that in general society looked down on people who had any kind of STI, resulting in the discreet behavior of patients and their families. Group participants said that many times people suffering from STIs did not share their problems with anyone including care providers due to fear of stigma and discrimination.

"Everyone looks down on people with STIs and no one wants to share anything with them. Therefore, the patient gets totally ostracized from the rest of the society and develops an inferiority complex."

Married migrant, Faisalabad

"Such people who suffer from these diseases should be told that they have committed a sin and are now a burden for the society. Why should everyone suffer through one person's fault. We all want to indulge in adventures but need to control ourselves as we have other responsibilities."

Married non-migrant, Peshawar

The unmarried males, especially in Karachi and Faisalabad, were vocal in their support of such people who had acquired any STI. Many young men felt that compassion and sympathy should be shown for these people.

"We should show compassion for people with AIDS or any such disease. Society should be supportive so that treatment can be extended for STI patients. After all, our religion also teaches us compassion for all."

Unmarried non-migrant, Karachi

There was a general feeling that stigma and discrimination issues were so prominent in this society due to lack of awareness. Most people generally felt that STIs could be spread through mere contact, such as shaking hands or sharing a meal or other utensils. There were suggestions from many groups that if public awareness was increased, issues of stigma and discrimination would go down.

\section{Sexual behavior}

Understanding the sexual behavior of the target groups was the crux of this research. In order to design any interventions for controlling STIs, it is crucial to know how men especially migrant men - behaved sexually. This is because men, specifically migrant men, are considered the bridge between the high-risk groups and the general population. Thus, men who have sex with sex workers or other sexual partners have the potential to transmit infections to their wives and other family members. 
This part of the study endorses the findings of the earlier 16 focus groups, namely, that promiscuous sexual behavior is quite common among Pakistani men. According to the participants, males upon reaching puberty (between the ages of 12 to 14 years) started experimenting in sex. Early exposure to sex-related material was quite common.

"Children of 11 and 12 are sexually active now. This is all because of media exposure. They start experimenting by viewing cable channels and pornographic movies. Masturbation leads to actual sexual acts and then continues in the same pattern."

Married non-migrant, Quetta

"I remember in our times a boy would reach puberty when he was 15 or 16 years old, and the same was true for girls. Nowadays, children as small as 9 or 10 know everything about sex and start looking for opportunities for sex. It is not only the media but the type of diet our children have these days. Then co-education is another issue that makes children more conscious about the opposite sex."

Married migrant, Faisalabad

Some of the participants felt that children in villages and those living in low-income localities received more exposure due to over-crowded living conditions.

"Children living in villages and congested localities are exposed to sex early in life because they see their parents having sex. Therefore, they discuss these acts with their friends and then start experimenting themselves."

Married migrant, Karachi

Friends and peer groups were another major source of exposure to sex. Young boys not only shared information with each other, but also ventured into sexual adventures within groups. Initial support from each other further encouraged youngsters to try the actual sexual act. Many times the information shared by young boys was based on myths and misconceptions.

The common sexual awareness stages recounted by the participants were initially masturbation, followed by experimentation with other young boys because females were not easily accessible for young boys, and then, later, sex workers. Several respondents also mentioned sex with animals, especially in rural areas, due to the limitation of female sexual partners. 
"Sex with animals is common in villages as they are easily accessible and there is no fear of being reported back by the donkey. Also, in some areas there are certain notions of "hot" and "cold" associated with sex with certain animal species, and people have their preferences accordingly."

Unmarried migrant, Faisalabad

"In our areas, boys usually have homosexual experiences early in life because there are no girls available for having sex. Some continue with the habit afterwards also, while others stop doing it after marriage."

Married migrant, Peshawar

Men, both married and unmarried, generally felt that sex was a natural phenomena and an integral part of human nature. Everyone had the need for sexual fulfillment after a certain age, and began indulging in sexual activities according to their circumstances.

"Once someone has had sex, he or she wants it again and again."

Unmarried non-migrant, Karachi

In the older married groups, some participants endorsed the concept of early marriages. They said that the main logic behind this was so young men would not stray and would learn to limit themselves to their wives.

"In order to avoid promiscuous and extramarital sex among men, boys should be married off after reaching adolescence, so that they do not indulge in bad sexual activities, and remain with their spouses only."

Married migrant, Peshawar

Extramarital sex was perceived to be quite common according to the participants. Different group members placed various proportions on the degree of the activity, ranging from 25 percent to as high as 90 percent. Within each group, there was variation in men's perceptions of extramarital sex: though most participants showed their disapproval for sex outside marriage, they conceded that it was a usual practice among most married men.

Many respondents, especially those at lower income levels, felt that extramarital sex depended on the economic status of the male. In Pakistan, women who are not sex workers are not easily accessible due to social norms; however, the services of a sex worker are not affordable to all. Many respondents mentioned that opportunities and availability of sex were issues. 
"Not all men have opportunities for sex outside their marriage due to many reasons. Availability and affordability are also problems, which prevent them from extramarital sex. Otherwise, the majority would have sexual relations with women other than their wives."

Married migrant, Quetta

Major influences or reasons given for sex outside marriage were primarily "the nature of males" and "boredom with wife." According to many respondents, men wanted variety in life and the same woman stopped exciting them after a while. Another common perception was that husbands could not sexually experiment with their wives; therefore, they went to other women with whom they could try new ways and means for sexual pleasures.

"One can't do sexual tricks with the wife or expect her to satisfy the husband through various other ways. So men go to a sex worker as she is prepared to excite you in different ways."

Married migrant, Peshawar

As the data on the background characteristics of the participants also show, married non-migrants and unmarried non-migrants mostly lived with their families, and therefore had less chances of availing sex outside their homes, as compared to migrant men who lived in bachelor quarters and had more exposure to such activities. Friends and media were also mentioned as main determinants for higher indulgence in sexual acts. People living with families naturally were limited in accessing such friends or viewing provocative channels in the presence of families.

Safe places for sex. Places for sex usually depended on the situation and the economic status of the person. Participants felt that those who had the means used hotel rooms for sexual pleasures, while the less affluent went to brothels. Students or unmarried men either used a friend's room or some private place, like a building under construction. According to a few respondents, cars were also used in cases where no other place was available.

"A friend's baithak [outside living room] is the ideal place for having sex."

(Unmarried non-migrant, Quetta 
Appendix

\section{Access to sex workers and concept of paid and unpaid sex}

Sex workers are easily available in all of the major urban centers of Pakistan. Certain localities are notorious for these activities and most men are aware of them. CSWs are available at various rate, ranging from Rs. 50 to 10,000 (approximately US\$ 1 to 200) or above for the night depending on the type of CSW and the client's economic circumstances.

In all of the focus groups, participants were able to identify localities in their respective cities where sex workers were present. Information about such localities was usually passed on through friends and colleagues. In several cases, participants said that CSWs could be recognized through their body language, and that in many instances a CSW would give a signal that the potential client recognized.

"Only immoral men recognize such women, as they learn to understand their body language and signs."

Married non-migrant, Faisalabad

According to several participants, the sex business had now altered so much that instead of the traditional brothels, CSWs could be contacted through the telephone and every detail could be fixed over the phone. Pimps were still an existing source for accessing a CSW. In addition, friends were a source for availing CSWs, as they had either personal experience or had learned from other friends, so it worked as a chain of information.

The majority of the participants felt that men who visited CSWs used multiple partners rather than limiting themselves to a single partner every time. 'Variety' was a main reason for having sex with a CSW; therefore, the majority went to a different woman each time.

"Such a man seldom visits the same woman every time, unless he develops an emotional relationship with her. For him a different woman each time is more exciting."

Married migrant, Karachi

Regarding the practice of paid and unpaid sex, all groups felt that it depended on the situation. Unpaid sex was carried out with friends who could be neighbors, colleagues, cousins, or class fellows. With these friends, where no cash was exchanged, then gifts were normally given as a compensation for sexual favors. Due to the prevailing sociocultural norms, such relationships are kept very discreet. 
All of the participants perceived extramarital sex among married women as highly condemnable. However, many participants said that it was practiced quite noticeably. The unmarried migrant groups in Peshawar said that 30 to 35 percent married rural women indulged in sexual relations outside their marriages.

"In some areas of our province, the men have gone to the cities for work reasons. So their wives naturally have the need for sex and indulge in such affairs. It is quite common and known."

Unmarried non-migrants, Peshawar

"Married women do indulge in extramarital sex. Nowadays there are some 'Aunties' who keep young girls with them and they are the dealers. In NWFP, there is no specific location like Hera Mandi. People are of Islamic mind."

Unmarried migrant, Peshawar

There were differing perceptions among many group members about female sexual practices in terms of sex before marriage and after marriage. There was consensus among the participants that the younger generation believed in much more sexual freedom, as compared to the generation before them, due to media exposure, coeducation and higher female employment.

"Girls now study with boys and work with men. So they have more chances of getting sexually involved. At least 40 to 50 percent girls are not virgins when they get married."

Married non-migrant, Quetta

Educated married migrants were less judgmental regarding extramarital sex among women.

"In our society, women are loyal and their upbringing does not permit them to have sex with anyone other than their husbands. This is a figment of people's imagination that a high percentage of married women have extramarital sex."

Married migrant, Karachi

\section{Men having sex with men}

In Pakistan, male and female interaction, outside of the immediate and extended family, is socially uncommon at most levels of society. Only in selected educational institutions do boys and girls get a chance to interact to a limited degree. Each gender forms its own separate group, while any male-female friendship is generally doubted and questioned. Initial sexual acts at early adolescence were said to be with male friends or other older male acquaintances. 
Some regions, like NWFP, are well known for homosexuality due to the closed cultural practices and segregation of females. Pathans themselves concede that homosexuality is more common in their province as compared to other regions.

"Because men do not have access to females they vent out their sexual frustrations by having sex with other men. Then there is also this element of exploitation of young labor class boys working as workshop workers and conductors, who are exploited by older men sexually and later become addicted to homosexuality."

Unmarried non-migrant, Peshawar

However, homosexuality was not approved of by the majority of focus group participants. Men in all categories did not accept it as a normal act and felt that it was mostly due to unnatural sexual inclinations and lack of opportunities for heterosexuality. Only a small number of respondents said that homosexuality was actually a matter of preference, that some men just preferred anal sex as compared to vaginal sex.

"For some men, anal sex is much more pleasurable as compared to conventional sex. Such men demand anal sex from sex workers also."

Married non-migrant, Karachi

On the question of oral sex, participants felt that it was an "imported" concept basically transferred through pornographic material through CDs and the Internet. According to the younger unmarried groups, it was still not very common in Pakistan and people usually only demanded oral sex from sex workers rather than regular sex partners or spouses.

Sex with hijras (transsexuals) was also mentioned as a means for sexual fulfillment; however, this was mostly perceived to be an activity on at the lower economic strata. Men felt that only men who did not have enough money to hire a female sex worker had sex with transsexuals, though some participants also thought that men who had regular sex with hijras did not like sex with anyone else.

"Men who have sex with hijras only like it with them. The pleasure they have in that kind of sex is not there for them with anyone else. Also, hijras are much more faithful and loyal, as compared to female sex workers, so some men develop steady relationships with them."

(Married migrants, Karachi 


\section{Awareness and prevention}

Unfortunately, the level of awareness about practices to help in preventing STI transmission during sex were lacking in the overall context. Although many participants mentioned the use of condoms, this was more oriented toward prevention of pregnancy rather than prevention of disease. Educated respondents were more aware of the use of condoms for prevention purposes, but agreed that many people did not use them due to lack of awareness and the urgency of the situation at the time of sexual contact.

"People might know that it can prevent them from disease, but at that time no one cares. They are always in such a hurry that the use of condom is overlooked."

Unmarried migrant, Faisalabad

In some cases, especially in certain localities of Karachi and Peshawar, it was reported that a few sex workers insisted that their clients use condoms before engaging in sex. Some men carried condoms with them for this purpose. However, most men felt that condoms reduced pleasure and could also inflict allergic reactions among some men. Several men said that there was no pleasure in sex unless there was "skin-to-skin" contact. Overall, with the exception of limited cases, FGD participants thought that condoms were not used by a large number of people.

"My friend used a condom once and developed a skin problem afterwards. They [condoms] are usually of low quality and also burst during sex, so then what is the point."

Married migrant, Faisalabad

Many respondents said that the most effective prevention of STIs was to adopt the Islamic principles of piousness and restricting oneself to one's spouse. Some participants also mentioned that in certain areas lady health workers promoted the use of condoms during sex, but they recommended their use mostly for family planning purposes.

The concept of bathing before sex was quite surprising for majority of the men, and was reported to be practiced only among those males who took a shower after returning from work, rather than for sexual purposes. However, there was a universal practice of taking a bath after sex. But the duration varied, while some bathed the morning after, others, following Islamic practice, showered immediately after sex. 
"Showering before sex is very seldom, but after sex it is essential, as our religion teaches us to do so as one needs to be clean, in case death comes and then one will die impure."

Married migrant, Quetta

\section{Health seeking behavior}

With growing awareness and various public and private sector health-related interventions, people are more conscious of their health needs and the requirement for quality treatment. Although the affordability and accessibility of health services still remain major issues for a large segment of the society, people understand the importance of going to proper doctors for treatments. This was also reflected in the responses of the FGD participants, who mostly suggested that allopathic treatment was comparatively more effective; however, due to various reasons, a significant number of people still preferred to go to traditional health providers, especially for treatment of STIs.

Many respondents said that people went to hakeems mostly for treatment of STIs: first, because they were more accessible, due to the advertisements specifically targeting STI cures; second, they were less judgmental; and third, because hakeems were cheaper.

"It is easier to visit hakeems for private problems because it is not as embarrassing talking to them, and also because hakeems are more affordable."

Married non-migrant, Faisalabad

Higher economic groups as well as those with better education preferred allopathic doctors.

"No one goes to traditional healers anymore, as people are more aware and understand that traditional healers can create further complications. Only the illiterate avail such services now."

Married migrant, Karachi

Homeopathic doctors and pirs were seldom visited for STI cures, probably due to the fact that pirs are decreasing in popularity in the cities, and homeopathic doctors are not commonly known for STI treatment.

Many men, especially those who are married, also used home or traditional remedies for STI treatment. 
"I have suffered from various urine infections and cured it by drinking lots of water. Certain foods also help in treatment of certain STIs."

Married migrant, Karachi

The unmarried males generally tend to share private problems with their friends, who then recommend the mode of treatment. While married men were mostly less inhibited and sought treatment earlier, rather than prolonging the problem and creating further complications.

"Many young boys either ignore the problem or delay going for treatment, which creates life-long complications like impotency and weak potency."

Unmarried non-migrant, Peshawar

\section{Concept of masculinity}

Only a few men associated honor, courage and power with masculinity; the remaining participants (both married and unmarried) linked the notion with "sexual power." The following quotes reflect the basic understanding of masculinity among the participants.

"A masculine man is he who can do sex for a longer duration and is able to satisfy his partner."

34 years old married graduate from Faisalabad A masculine man is he who can produce children."

30 years old graduate Unmarried from Karachi

"A masculine man is he who has a big and strong sexual organ."

21 years old un-married intermediate from Faisalabad

Similarly, a feminine or 'na mard' male was perceived as an impotent man, someone who had weak sexual potency and was unable to satisfy his woman.

"A na mard is he who cannot perform sexually and is unable to satisfy his woman. Men who suffer from premature ejaculation are also na mard.

Unmarried migrant, Faisalabad

\section{Drug abuse, blood transfusion and tattooing}

Drug abuse is common in Pakistan. A significant portion of the male population indulge in chewing tobacco, eating pan leafs and smoking. A growing menace is gutka chewing (a mix of tobacco and beetle nuts), which is very popular in Sindh and parts of Balochistan, with even young children addicted to it. On the whole, most participants were aware of the dangers of tattooing, and of used blades and syringes. 
According to FGD participants, drug abuse was quite common. As in the sex trade, certain localities were known for drug peddling. Charas, or hashish, and alcohol were the most common among men; some women also indulged in these. Fortunately, injecting drug use was not as common as feared, and only certain segments used substances like heroin and opium injections. However, tranquilizer shots were gaining in popularity; they are more available than heroin, which is difficult to buy and costly.

"Ninety percent of people use charas and most are students, especially those of medical and engineering sciences. Medical students use it more often because they are tense. Pathans mostly use naswar while Punjabis use cigarettes. Heroin use has become very uncommon now."

Unmarried migrant, Peshawar

The participants were of the opinion that very few drug addicts indulged in sexual activities or sold sex for buying drugs because addicts had no sexual inclinations, they just wanted to sustain their habit.

There was quite a high level of awareness about blood screening and the possibility of hepatitis or HIV/AIDS and other infections through unscreened blood. However, participants in most groups felt that the blood-screening system in the country was not regulated, especially in the private hospitals.

"Even these blood banks do not do any blood screening. So we just use the blood of those we know in case of an emergency."

Unmarried non-migrant, Karachi

According to many respondents, the quality of blood screening depended on the place where one went. Many reported that their experience had been positive, saying that when they had gone for blood donations, it had been screened first; others, however, told stories that reflected the opposite experience.

Tattooing was not a common practice, nor was it much appreciated. Tattooing was perceived to be on the decline. Respondents thought it was only practiced in certain groups or times (e.g., mostly young men or during village carnivals, etc.).

"A very few young boys have their beloved's name engraved somewhere on their bodies. Otherwise, it is not very popular."

Unmarried migrant, Quetta 


\section{APPENDIX 4. TEAM MEMBERS}

\section{Population Council Core Study Team}

Dr. Zeba A. Sathar, Overall Study Supervisor

Dr. Ali M. Mir, Principal Investigator

Dr. Laura Reichenbach, Consultant

Dr. Abdul Wajid, Dy. Principal Investigator/ Study Coordinator

Mumraiz Khan, Dy. Study Coordinator/Assistant Princial Investigator

Muhammad Irfan Masood, Data Quality Manager

Mansoor Qaisar, Study Secretary

Ms. Tayyaba Gul, Logistic Support Officer

\section{Research Consultants}

Dr. Syed Abdul Mujeeb

Dr. Steve Pearson

Ms. Sarah Javaid

\section{Study Secretariat/Control Room Team}

Faisal Shah

Zulfiqar Hussain

Mohsin Nadeem

\section{Admin \& Finance Support Team}

Mr. Imran Ahmad,Director Programs, Population Council

Mr. Badar ul Islam,Accounts Officer, Population Council

Mr. Abdul Kashif,Accounts \& Admin Assistant 


\section{City Wise Field Teams}

\section{Faisalabad}

\section{Team 01}

Dr. Shahid Iqbal, Medical Doctor and

Field Team Leader

Muhammad Baber Akram, Facilitator

Irfan Mahmood, Interviewer

Imran Ibrahim, Interviewer

Muhammad Amir, Laboratory

Technician

Jawad Hussain, Phlebotomist

\section{Team 02}

Dr.Qamar-ul-Haq, Medical Doctor and

Field Team Leader

Muhammad Mahmood Akhtar,

Facilitator

Nasir Bashir, Interviewer

Javed Iqbal, Interviewer

Umar Farooq, Laboratory Technician

Arfan Muhamood, Phlebotomist

\section{Karachi}

\section{Team 01}

Dr. Tajjudin Malik, Medical Doctor and

Field Team Leader

Dr. Waqar Ahmed, Facilitator

Hamad Ahmad, Interviewer

Noor Hassan, Interviewer

Mohammad Nasir, Laboratory

Technician

Arshad Ali Khan, Phlebotomist

\section{Lahore}

Dr. Asif Mehmood, Doctor

Ali Raza, Facilitator

Aurangzeb, Facilitator

Ghulam Mustafa, Interviewer

Faisal Shah, Interviewer

Mr. Zulfiqar Hussain, Interviewer

Mr. Mohsin Nadeem, Interviewer

Ahmed Hassan, Interviewer

\section{Team 02}

Dr.Anzar Rizvi, Medical Doctor and

Field Team Leader

Jamal Saeed, Facilitator

Dr. Fahim Hakro, Interviewer

Hafeez ur Rehman, Interviewer

Abdul Rehman, Phlebotomist

\author{
M. Babar Aakran, Interviewer \\ Muhammad Mehmood Akhtar, \\ Interviewer \\ Javaid Iqbal, Interviewer \\ Imran Ibrahim, Interviewer \\ Ehstasham Babar, Interviewer \\ M.Latif, , Phlebotomist \\ Akhlaq Ahmed Imran, Phlebotomist
}




\section{Rawalpindi}

\section{Team 01}

Dr.Abdul Sattar, Medical Doctor and

Field Team Leader

Ahtesham Babar, Interviewer

Abdul Rasheed, Interviewer

Ahmed Hassan, Interviewer

M.Yasir Qureshi, Laboratory

Technician

Akhlaq Ahmed Imran, Phlebotomist

\section{Peshawar}

\section{Team 01}

Dr.Abdul Hadi, Medical Doctor and

Field Team Leader

Shakeel Ahmed, Facilitator

Syed Ali Hussain, Interviewer

Atiq-ur-Rehman, Interviewer

Tanzeem Hassan, Laboratory

Technician

Sajjad Hussain, Phlebotomist

\section{Team 02}

Dr. Arshad Sabir, Medical Doctor and

Field Team Leader

Ali Raza, Interviewer

Ghulam Mustafa, Interviewer

M.Aurangzeb, Interviewer

M.Nauman, Laboratory Technician

M.Latif, , Phlebotomist

\section{Team 02}

Dr. Usman Ahmed Raza, Medical Doctor and Field Team Leader

Taimur Khan Afridi, Facilitator

M.Najmul-Aleem, Interviewer

Syed Faiq Sajjad Shah, Interviewer

Askar Abbas, Interviewer

Javid Iqbal, Interviewer

Rehmatullah, Laboratory Technician

Mr. Saleem , Phlebotomist

\section{Quetta}

\section{Team 01}

Dr.M.Ilyas, Medical Doctor and Field

Team Leader

Shafiq ur Rehman, Facilitator

M.Saddique, Interviewer

Abdul Razique, Interviewer

Syed Naqeebullah, Laboratory

Technician

Ghulam Akbar, Phlebotomist

\section{Team 02}

Dr Rafiushan, Medical Doctor and Field

Team Leader

M.Ayub Akhtar, Facilitator

Zahoor Ahmed, Interviewer

Muhammad Qasim, Interviewer

Abdul Latif Pirkani, Laboratory

Technician

M. Naseem, Phlebotomist

\section{Data Entry Team}

Muhammad Ali

Mazhar Abbas Shah

Sohail Tabassum
Saifullah

Muhammad Wasif

Muhammad Usman Ghani 



\section{APPENDIX 5. ROLES AND RESPONSIBILITIES OF EACH TEAM MEMBER}

\section{Medical Doctor}

- Generally the medical doctors will be the overall supervisors of the study team and they will assist guide and support all team members and will ensure that the fieldwork is carried out according to the high ethical standards, methodically and within time.

- The medical doctor will identify and set up a base camp at a point within the PSU that offers visual and auditory privacy and maintain Confidentiality.

- He will help in supervising the lab technician to set up his field lab.

- He will provide general medical care to all the study respondents and also syndromic management of sexually transmitted infections (STIs) for those who may be in need of.

- He shall oversee data entry and along with the field supervisor and conduct on spot site visits.

- He shall organize the logistics of the team-ensuring adherence to one field plan.

- He shall oversee the work of the lab technician and make sure that all specimens are stored and dispatched by the lab technicians scrupulously following the standard operation procedures.

- He shall receive all results in sealed envelope sent by the Population Council and handover these to the respondents who can collect these on the production of their PIN numbers (personal identification number).

- He will refer all cases requiring expert medical attention to tertiary level care facilities where arrangement will have been made for fast tracking cases.

- He shall provide on spot results of the syphilis rapid results and suggest management including partner management. 


\section{Supervisor/Facilitator}

- The social scientist supervisor will in discussion with the medical doctor develop a daily work plan and make transport arrangements accordingly.

- The supervisor will facilitate in identification of the households in collaboration with FBS employee.

- He will assist in decisions related to replacement of household.

- He will himself conduct one interview per day and if applicable the in-depth interview as well.

- He will monitor the fieldwork in terms of maintenance of upholding ethical principles and ensuring high quality of data collection.

- He will be responsible for the safety and security of the field teams.

- He will collect all questionnaire and check for completeness.

- He will maintain all receipts and financial records and submit these to the Population Council office.

- He will maintain a field survey diary and record his notes.

- He will on a daily basis provide a daily report to the Population Council Control Room.

- He will submit the following material to the Population Council by TCS

1. Log sheet one copy

2. Filled questionnaire

3. Daily status sheet

4. In-depth interviews status sheet

\section{Social Scientists (interviewer for behavioral questionnaire)}

- Will obtain informed consent.

- Ensure maintenance of privacy and confidentiality.

- Develop rapport and obtain the trust of the respondent

- Fill in the questionnaire in a friendly and non-threatening manner.

- Ensure all sections of the questionnaire are filled in completely. 
- Ensure that household-listing number is provided to phlebotomists and it is entered into the sticker codebook.

- Ensure that questionnaire sticker has been obtained from phlebotomists and affixed to the questionnaire.

\section{Role of phlebotomist and lab technician}

- The phlebotomist will accompany the social scientist in the field and collect blood and urine samples

- He will enquire from the social scientist the household listing number and enter it into the sticker codebook.

- The phlebotomist will maintain the sticker codebook.

- He will collect the sample using a vacutainer and the blood will be injected directly into two blood tubes- one having the anticoagulant and one without, immediately the tube with the red top will be affixed with the red color sticker and the second tube with the mauve colored top will be have the mauve colored sticker affixed to it.

- He will affix the pink color sticker on to the DBS card.

- Urine samples will be collected in sample container and yellow color sticker will be applied

- He will than affix the white color sticker on to the respondent result card.

- He will carry the specimens as quickly as possible to the central camp site where the medical doctor and lab technician will be available

- He will affix the two blue colored stickers on to the two log sheets

- He will hand over the specimens and the DBS card to the lab technician

- Lab technician following the standard operating procedures (SOP's) will prepare DBS cards and will perform syphilis rapid test

- Lab technician will be responsible for the disposal of all sharps and gloves, etc.

- Lab technician will properly store the specimens in the ice pack and prepare the consignment for transport observing all safety precautions

- Lab Technician will fill in the two log sheets

- Lab technician will personally deliver the specimens to the courier service 



\section{APPENDIX 6. IN-DEPTH INTERVIEW (IDI) GUIDE}

In-depth interviews will be carried out with all respondents who report having had sex with someone other than their wife in the last three months.

The purpose of these IDIs is to obtain in-depth information about sexual behavior and other potentially risky behavior that help to identify the bridging population among urban men in Pakistan. In particular, the IDIs will elucidate how the respondent selects their sexual partner, how they make their arrangements for sex, and whether or not he pays for sex in cash or in kind.

If you wish we would like to seek some further information on the same questions we have asked you before. The process will take about 40 minutes. Your participation is voluntary and there is no penalty for refusing to take part. Your answers will be kept completely confidential. You may refuse to answer any question in the interview or to stop the interview at any time.

The following introduction and informed consent will be taken in these cases:

"This interview is being conducted to get more detailed information about men's health issues. I am especially interested in your preferences and behaviors related to health seeking for reproductive health problems and also your sexual behavior."

"If it is okay with you I will be tape recording our conversation. The purpose of this is so that I can get all the details but at the same time be able to carry out an attentive conversation with you. I assure you that all your comments will remain confidential. I will be compiling a report without any reference to individuals. The tape recording will be erased as soon as we have completed our report. If you agree to this interview (and the tape recording), please sign this consent form."

Note: If the respondent declines the tape recorded interview tell them that they can participate without being recorded. Informed consent will still be required. 


\section{Guidelines for IDIs}

\section{Topic: Educational and professional goals}

Main Questions:

- Can you tell me what level of education have you attained?

Follow-up Questions:

- Do you wish you had more education?

- If yes, can you tell me why you discontinued your education?

\section{Topic: Family structure}

Main Questions:

- How do you send your leisure free time?

- Who decides ho you spend your leisure free time (Yourself/friend/ family)

- Do you need to seek permission from anyone in your household to leave home and/or stay away from home until late?

If migrant (living away from marital or natal home for greater than six months):

- I know that you have lived away from your marital or natal home for months/years. Can you tell me a bit more about why you moved away from home?

- How often do you travel back to visit your marital/natal home?

- Do you think there are advantages to living away from home? What are these?

- Do you think there are disadvantages to living away from home? What are these?

- By living away from your home/family do you feel more autonomous/free to make decision on your own

\section{Topic: Sociodemographic information and personal habits}

\section{Main Questions:}

- Can you tell me about the kind of work you do? F/U or probe: Is it in an office or out in the field? Is it regular work? What skills or training do you have?

- Is this the job you want to have? What are your future professional goals? 
- Do you have a lot of friends? How often do you socialize with your friends? What do you do with your friends when you meet? \{e.g., eating out, watching movies, playing sports\}

- Do you ever use alcohol or drugs? If yes, can you tell me a little bit more about the situations in which you use alcohol or drugs? F/U or probes: do you do this alone or with friends or others? How often do you use alcohol or drugs? Which kinds? Where do you get them? How do you pay for them? Why do you think you use alcohol or drugs?

- Do you have a computer with internet access in your home?

- Do you access the internet? Have you ever used the internet to access pornography? Follow-up/probes: When and how often? Were you by yourself or with friends/others?

If migrant:

- What do you do in your spare time?

- Who do you like to spend time with when you have spare time?

\section{Topic: Reproductive health seeking behavior}

Main Questions:

- Can you tell me what you know about STIs or reproductive health problems in men?

- What would you think if one of your friends told you he had an STI?

- Do you feel comfortable talking about STIs or HIV/AIDS with anyone? F/U: Whom?

- Do you feel that you are at risk for acquiring an STI or HIV/AIDS? F/U: Why? Why not?

- Do you know where to go to seek treatment if you had an STI or symptoms related to your sexual organs? F/U: Who would you go to? Why?

- Do you have suggestions for improving access to reproductive health services or information about STIs?

- Who would you feel most comfortable receiving information about STIs/HIV/AIDS? F/U question: How would you most prefer to get this information? \{written materials, television, radio, from doctor, from friend or family member\}

\section{Topic: Sexual behavior}

"This is a polite request for information about the three most recent partners (?). Please speak frankly, we will not disclose what you tell us to anyone. We would like to begin by 
discussing your most recent partner and then the two just before the most recent partner"

Opportunities for engaging in sexual activities:

Main questions:

- Who was your most recent partner?

- Was this a male or a female?

- Did you know this person before?

- What is their relationship to you? \{friend, CSW, other\} Where did you first meet this person?

- How long have you been sexually involved with this person?

- How long did you know this person before engaging in sexual activity with him/her?

- How many times have you had sex with this person in the past month?

- As far as you know does this person have other sexual partners?

- Is this person economically better or worse off than you are?

- Did you give this person any money or other items in exchange for sex? F/U: How much cash or what other forms of payment? Do you always pay this person for sex?

"Now I would like to ask you some questions concerning the types of sexual activities that you and this person have experienced together. I realize that this is very personal information that you would normally not want to discuss with a stranger. The reason that we are asking you these questions is so that we can design more effective health programs and health education for men in your community. We will not succeed in our efforts without the participation and cooperation of people like yourself. Please try to answer the questions as candidly as possible. This information is strictly confidential and your name will not be revealed to anyone. Your name will not appear on any of our reports - everyone is identified by number only."

\section{Types of sexual activity}

Main questions:

- What types of sexual activity did you and this person engage in? \{Probes: anal, oral, vaginal, thigh sex\}

- Where did you have sex on this particular occasion?

Repeat set of questions for previous 2 sexual partners.

Motivations 
- Why do you engage in sexual activities with partners other than your wife or outside marriage\{Possible probes: more satisfying; can perform different activities; as part of a group activity; peer pressure; easily available; experimentation\}

- How do you prefer to locate a sexual partner (both paid and unpaid). F/U or probes: How do you use the mobile phone to locate sexual partner? Networks of friends?

- Do you consider yourself to be at risk of acquiring a disease as a result of your sexual practices

- While having sexual intercourse with a FSW what are the risks associated with such an activity.

- In case of having sex with a female friend what do you think are the associated risks

- In case of having sex with a male what do you think are the associated risks

- Before you have sex do you ever drink alcohol or use drugs?

- In your opinion, what are the advantages and disadvantages of a temporary sexual partnership?

- In your opinion, what are the advantages and disadvantages of a steady sexual partnership?

Condom use

\section{Main Questions:}

- Have you ever used a condom? Why? Or why not?

- In which situations do you use a condom? Why?

- When do you choose not to use a condom? Why?

- Do you use a condom every time you have sex? Why or why not?

In case there is a history of having sex with male and female please ask the following questions

If given a choice to have sex with a male or female whom will you give preference to? 



\section{APPENDIX 7. FOCUS GROUP DISCUSSION (FGD) GUIDE}

\section{Informed Consent for Focus Group Discussion}

\section{Consent statement for behavioral component}

Assalam-o-Alaikum

\section{Introduction:}

[Please read the statement below out loud before continuing with the questionnaire]

Assalamm-o-Alaikum. My name is I am working with an organization

called the Population Council that is helping the government of Pakistan to design health programs in Pakistan. We are interviewing men (single and married) living in urban areas either with or away from their families. In order to improve health services for men living in urban areas we need to know more about the health problems faced by men in particular. I am here today to talk with you and to gather information in general about health and illness with particular reference to sexually transmitted infections and sexual behaviors in men.

Your participation in this discussion is voluntary and there is no penalty for refusing to take part. No one except the moderator and the other group members will know that you took part in the research. The group discussion will be tape recorded with voices only. After transcription the audiocassettes will be erased. When the results of the research are published your name will not be shown.

The information you shall provide will be kept completely confidential. Your name will not be recorded and will never be used in connection with any of the information you tell me. You are requested not to reveal outside the group information may have heard during the discussion. You don't have to answer any questions or express your views on any topic that you do not want to do so and you can leave this discussion at any time you want to. However, your participation in this discussion will help us better understand what people think, say and do about certain kinds of behaviors. 
There is a small chance that what people talk about in the group will make you feel uncomfortable. There is also a small chance that others in the group may tell someone you were taking part or report what you said.

We would greatly appreciate your participation in this discussion. It will take 45-50 minutes. Are you willing to participate?

I have orally read the consent form to the research participants, and they have fully agreed to be in this focus group research. I further agree to keep confidential any ting that is said in the discussion group

Please print clearly:

(Signature of Moderator)

(Moderator's name)

Date

6 to 8 participants in each group:

Four categories of participants (16 to 45 years)

- Married migrants

- Unmarried migrants

- Married non-migrants

- Unmarried non-migrants

Points for the Moderator:

- Please make sure that you probe each point

- The discussion should be focused on the behavior and practices of the target group and its representative community. For example during the FGD with married migrant men, we want to know about married migrant community only;

- We want to know WHY, WHERE, WHEN and HOW according to the relevance of the point being discussed.

- Sum up each section for the benefit of the participants, the note-taker and yourself.

- Time management is very important and irrelevant issues should be (politely) ignored and put aside. The moderator can politely tell the participants that the issue is not relevant to the focus of discussion and can be discussed later if felt necessary by the participants 
- The Glossary can also be completed through a key informant in the target community or towards the end of the FGD; Glossary should not be over-looked as it is an important part of the study

\section{Points for the Note-taker:}

- All discussion points should be noted down

- Each participant should be labeled (A,B,C, D and so on) so that issues under discussion can be labeled according to the responses of the participants through out the discussion

- Emphasis of the note-taker should be on the discussion points according to the guidelines;

- The note-taker should also observe the body language and diction of the participants and note them down.

\section{Introduction to the subject matter and purpose of the study by the moderator}

\section{Background Characteristics of each participant:}

Each participant should fill up a form circulated by the research team or the note-taker should help those participants who are illiterate or unable to fill up the form themselves.

\section{Family patterns and trends of the target population}

- Do men live with their families, alone, or share living quarters with other men;

- What are the living conditions like: how many rooms, condition of kitchen, toilet etc. (Not individual cases, but general conditions are required);

- General employment and income levels of men (target group) in the area;

- Travel frequency as part of work, recreation.

\section{Level of awareness and knowledge regarding HIV/AIDS and other STIs}

- What are the commonly occurring Sexually Transmitted Infections (STIs); what are their symptoms, common mode of transmission, treatment? Please note down local terms of various STIs as mentioned by the participants

- Level of awareness about HIV and AIDS; what is it, what are its symptoms, mode of transmission, treatment?

- How can STIs and HIV be prevented?

- Sources of information

- Risk perceptions of the participants as to who is more vulnerable or at risk of getting STIs and HIV/AIDS

- Do the participants feel that age, education or occupation of people make them more or less vulnerable towards STIs/HIV/ AIDS

- Sources of information?

- Are their any cases of HIV/AIDS and STIs in their workplace, residential localities or anywhere?

- Issues of stigma and discrimination regarding HIV/AIDS and STIs; Due to stigma and discrimination issues, do people avoid disclosing their problems, or availing treatment; 


\section{Sexual Behavior}

- At what age do young males usually begin having sexual relations;

- What are the reasons that influence men into having sex before marriage

- Is sex before marriage common? Before marriage with whom do men usually have sex with? Trends towards paid and unpaid sex - which is more common in married and unmarried men and the kind of opportunities present - In case of unpaid sex, who is the most common partner. In case of paid sex where are the specific localities men frequently visit for availing sexual recreation with females, males and transgender.. (Names of localities where CSWs are available)

- While having sex do men use any preventive measures (like condoms) do these behavior change with partners, e.g., male and female and with paid and unpaid sex partners

- When and why are condoms used;

- Where can condoms be obtained?

- Are they used commonly and if not than why.

- How common is homosexuality? Why do some men indulge in this activity, Is it related to age, cost, availability, cultural, how and where are homosexual activities availed are they mostly paid or unpaid; Is it related to marital status Can married men also indulge in this activity WHY?

- Do women also have out of marriage sexual relations - trends among married and unmarried women - Reasons for such behavior according to the group; Community perceptions about out of marriage sex among females?

- Some men prefer visiting transgender workers what are the reasons. How common is anal and oral sex and why.

- Types of sexual behavior associated with different partners. (Oral/anal etc, male female).

- After marriage some men may engage in sexual relations with other women or sex workers, what are the common characteristics of these men (e.g., living away from their families.)

- What factors influence sexual behaviors, e.g., role of family, religion, location, exposure to media, peer pressure.

\section{Drug Abuse}

- How prevalent are drug being used in their area or place of employment? What kinds of drugs are common e.g. Alcohol? (Note: Focus is on injecting drug use and do not indulge in unnecessary discussion on other substance abuse with the group participants).

- Is injecting drug abuse common? Do people reuse syringes?

- Level of awareness regarding the harms of reused syringes?

- Is it easy to buy drugs? How expensive is it? (Focus on injecting drugs)

- Do people who indulge in drug abuse also indulge in sexual activities?

\section{Health Seeking Behavior}

- Where can people go for treatment of STIs and HIV/AIDS? (Govt versus private)

- With whom do they usually discuss such illnesses 
- Why people hesitant to visit doctors or treatment facilities for treatment of STIs due to sociocultural reasons?

- Why there a trend of men visiting Hakeems and traditional healers especially for male reproductive health issues/STIs or is there a preference for allopathic service providers (in case of sexual problems)

- Is there a certain preference for particular type of service provider? Reasons for the preference

- What are the factors that influence treatment seeking behavior of men for their reproductive and sexual health problems (costs, confidentiality, quality, e.g. Privacy, assurance provided by provider, provider's behavior etc.

\section{Role of Media}

- How effective or negative has been the role of media in creating awareness regarding Reproductive health issues including HIV/AIDS etc.

- Which media is most popular (TV, radio, internet, Print media)

- Is it common to have access to pornographic material and how popular is it among different age groups;

- Which source of pornographic material are commonly accessed. Do people know about telephone hotlines?

- Do people have access to Internet, for what purpose is it used. Is it used for accessing pornographic materials.

- Does the group feel that media affects peoples behavior and attitudes towards $\mathrm{RH}$ and sexuality?

\section{Others}

- Trends of blood donation in the community - awareness regarding blood screening or testing;

- How commonly is blood sold

- Is tattooing common - where do people get tattoos and from whom (general conditions in the tattooing place)

\section{Local Glossary of Specific terms:}

- Names for different STIs

- Local term for HIV/AIDS (if any)

- Sexual intercourse/ sexual act

- Homosexuality active and passive person

- Anal sex

- Oral sex

- Sexual weakness

- Infertility

- Self medication

- Quack

- Male sex workers

- Female sex workers

- Child sex workers 
Appendix

- Male homosexual partner active penetrator and passive

- Condoms

- Sex with animals

- Social stigma

- IV Drugs

- Tattooing

- Sexual pleasure

- Genitalia/ Genital region in males 


\section{APPENDIX 8. STEPS IN FIELDWORK}

\begin{tabular}{|c|c|c|}
\hline \multicolumn{3}{|c|}{ Steps to be followed in the field } \\
\hline Steps & Activity & Responsibility \\
\hline \multicolumn{3}{|c|}{ Activities on the interview site } \\
\hline 1 & Identification of Household in the block & Facilitator \\
\hline 2 & Identification of space for establishing lab & Facilitator \& Medial doctor \\
\hline 3 & $\begin{array}{l}\text { Movement to the identified household, roster } \\
\text { filling and start of interview after informed } \\
\text { consent }\end{array}$ & First social scientist \\
\hline 4 & $\begin{array}{l}\text { Entry of phlebotomist and greet the } \\
\text { respondent }\end{array}$ & Phlebotomist \\
\hline 5 & $\begin{array}{l}\text { Brief sharing of procedures for collection of } \\
\text { samples with the respondent }\end{array}$ & Phlebotomist \\
\hline 6 & $\begin{array}{l}\text { Writing PIN code, just below the green PIN } \\
\text { code sticker on the questionnaire, with } \\
\text { permanent pen }\end{array}$ & Phlebotomist \\
\hline 7 & Providing respondent card with PIN number & Phlebotomist \\
\hline 8 & $\begin{array}{l}\text { Applying red sticker on red top tube and } \\
\text { purple sticker on purple top tube }\end{array}$ & Phlebotomist \\
\hline 9 & Washing hand with scrub & Phlebotomist \\
\hline 10 & Putting on gloves & Phlebotomist \\
\hline 11 & Applying of tourniquet & Phlebotomist \\
\hline 12 & $\begin{array}{l}\text { Identification of vein and cleansing the site } \\
\text { with antiseptic swab }\end{array}$ & Phlebotomist \\
\hline 13 & $\begin{array}{l}\text { Opening of needle by twisting antilock wise } \\
\text { upper portion and breaking the seal. }\end{array}$ & Phlebotomist \\
\hline 14 & $\begin{array}{l}\text { Putting the needle in the holder with needle } \\
\text { outside }\end{array}$ & Phlebotomist \\
\hline 15 & Pricking the vein. & Phlebotomist \\
\hline 16 & $\begin{array}{l}\text { Connecting the purple top tube to the rear end } \\
\text { of needle through holder and filling in the tube } \\
\text { with } 4 \mathrm{mls} \text { of blood. Withdrawing the tube. }\end{array}$ & Phlebotomist \\
\hline 17 & $\begin{array}{l}\text { Connecting the red top tube to the rear end of } \\
\text { needle through holder and filling in the tube } \\
\text { with } 4 \mathrm{mls} \text { of blood. When tube is getting }\end{array}$ & Phlebotomist \\
\hline
\end{tabular}




\section{Steps to be followed in the field}

\begin{tabular}{|c|c|c|}
\hline Steps & Activity & Responsibility \\
\hline & $\begin{array}{l}\text { filled; moving the red top tube up and down to } \\
\text { mix the contents with the blood } \\
\text { (as shown in fig. 1). }\end{array}$ & \\
\hline 18 & Withdrawing the purple top tube. & Phlebotomist \\
\hline 19 & Removing the tourniquet & Phlebotomist \\
\hline 20 & $\begin{array}{l}\text { Withdrawing the needle with gentle pressure } \\
\text { of dry cotton piece. }\end{array}$ & Phlebotomist \\
\hline 21 & $\begin{array}{l}\text { Advising the respondent to fold the arm at } \\
\text { elbow joint if blood drawn from veins on } \\
\text { elbow joint. }\end{array}$ & Phlebotomist \\
\hline 22 & $\begin{array}{l}\text { Moving the red top tube up and down to mix } \\
\text { the contents with the blood (as shown in fig 2). }\end{array}$ & Phlebotomist \\
\hline 23 & $\begin{array}{l}\text { Discarding the sharps in sharp disposal yellow } \\
\text { box. Use this box till it is filled / full. }\end{array}$ & Phlebotomist \\
\hline 24 & $\begin{array}{l}\text { Discarding the other wastes in the red large } \\
\text { bag. Use it until space is left to accommodate } \\
\text { yellow disposal box. }\end{array}$ & Phlebotomist \\
\hline 25 & $\begin{array}{l}\text { Unfolding the arm of the respondent, } \\
\text { removing the cotton piece and application of } \\
\text { saniplast on punctured site. }\end{array}$ & Phlebotomist \\
\hline 26 & $\begin{array}{l}\text { Placing the tubes in the plastic container } \\
\text { padded with cotton layers at room } \\
\text { temperature. }\end{array}$ & Phlebotomist \\
\hline 27 & $\begin{array}{l}\text { Applying the green top plastic bottle for urine } \\
\text { collection with yellow sticker }\end{array}$ & Phlebotomist \\
\hline 28 & $\begin{array}{l}\text { Handing over urine collection container to the } \\
\text { eligible respondent for urine sample. Advising } \\
\text { the respondent to fill the bottle with } 20-40 \text { mls } \\
\text { of urine of first stream. Respondent should not } \\
\text { have passed urine for more than one hour. }\end{array}$ & Phlebotomist \\
\hline 29 & Thanking the respondent. & Phlebotomist \\
\hline 30 & Completion of interview & Social Scientist \\
\hline 31 & Thanking the respondent. & Social Scientist \\
\hline 32 & $\begin{array}{l}\text { Providing respondent's card with PIN code } \\
\text { affixed on it }\end{array}$ & Social Scientist \\
\hline
\end{tabular}


Steps to be followed in the field

\begin{tabular}{|c|c|c|}
\hline Steps & Activity & Responsibility \\
\hline 33 & $\begin{array}{l}\text { Providing letter with information on how, } \\
\text { when and from where receive the results. }\end{array}$ & Social Scientist \\
\hline 34 & Providing VCT centers list & Social Scientist \\
\hline 35 & Providing IEC material prepared by NACP. & Social Scientist \\
\hline 36 & $\begin{array}{l}\text { Transporting all the samples to the lab } \\
\text { technician immediately }\end{array}$ & Phlebotomist \\
\hline \multicolumn{3}{|c|}{ Activities in the field lab } \\
\hline 37 & $\begin{array}{l}\text { Writing PIN code with permanent ink on } \\
\text { Rapid Syphilis Test strip }\end{array}$ & Lab tech \\
\hline 38 & Affix pink color sticker to DBS card & Lab tech \\
\hline 39 & $\begin{array}{l}\text { Affixing one blue sticker each on the two log } \\
\text { sheets. }\end{array}$ & Lab tech \\
\hline 40 & $\begin{array}{l}\text { Filling in the log sheets according to the } \\
\text { availability of the samples present. }\end{array}$ & Lab tech \\
\hline 41 & Hand washing with disinfectant. & Lab tech \\
\hline 42 & Putting on gloves. & Lab tech \\
\hline 43 & Fixing the pipette at $50 \mu \mathrm{l}$ (micro litter) level. & Lab tech \\
\hline 44 & $\begin{array}{l}\text { Sucking } 50 \mu \text { l blood from purple top bottle and } \\
\text { placing it on the white pad on } \\
\text { Syphilis Rapid Test strip. Avoid touching the } \\
\text { pad on strip with the tip of pipette }\end{array}$ & Lab tech \\
\hline 45 & Waiting for one minute & Lab tech \\
\hline 46 & $\begin{array}{l}\text { Putting one drop of buffer reagent on the blood } \\
\text { already put on the strip. }\end{array}$ & Lab tech \\
\hline 47 & Waiting for 15 minutes after adding buffer. & Lab tech \\
\hline \multirow[t]{2}{*}{48} & $\begin{array}{l}\text { Drawing another } 50 \mu \mathrm{l} \text { of blood from purple } \\
\text { top bottle and placing one drop on each of the } \\
\text { five circles drawn on DBS card. }\end{array}$ & Lab tech \\
\hline & $\begin{array}{l}\text { Drying DBS card in air; red color of blood will } \\
\text { turn brown }\end{array}$ & Lab tech \\
\hline 49 & Reading the result of syphilis rapid test. & Lab tech \\
\hline 50 & Interpretation of results (as shown in fig 3). & Lab tech \& Medical doctor \\
\hline 51 & Recording the result on log sheet & Lab tech \\
\hline 52 & $\begin{array}{l}\text { Providing services including the medicines to } \\
\text { the respondent when he arrives for receiving }\end{array}$ & Medical doctor \\
\hline
\end{tabular}




\begin{tabular}{|c|c|c|}
\hline \multicolumn{3}{|c|}{ Steps to be followed in the field } \\
\hline Steps & Activity & Responsibility \\
\hline & his result of syphilis. & \\
\hline 53 & $\begin{array}{l}\text { Filling in OPD form for diagnosis and } \\
\text { treatment given with quantity of medicines } \\
\text { provided. }\end{array}$ & Medical doctor \\
\hline 54 & $\begin{array}{l}\text { Wrapping blood tubes in three layers of } \\
\text { absorbent towel; both tubes should be } \\
\text { wrapped separately. }\end{array}$ & Lab tech \\
\hline 55 & $\begin{array}{l}\text { Placing cotton wool in the bottom of the plastic } \\
\text { container. }\end{array}$ & Lab tech \\
\hline 56 & $\begin{array}{l}\text { Placing these tubes in the plastic container. } \\
\text { This container can accommodate } 10-15 \text { tubes. }\end{array}$ & Lab tech \\
\hline 57 & $\begin{array}{l}\text { Affixing BIOHAZARD label on this plastic } \\
\text { container. }\end{array}$ & Lab tech \\
\hline 58 & $\begin{array}{l}\text { After securely fixing the lid of urine container, } \\
\text { place this container in zip lock. }\end{array}$ & Lab tech \\
\hline 59 & Closing the zip lock firmly. & Lab tech \\
\hline 60 & $\begin{array}{l}\text { Placing four-chilled ice pack in the Styrofoam } \\
\text { box. }\end{array}$ & Lab tech \\
\hline 61 & $\begin{array}{l}\text { Placing the zip lock in Styrofoam box with ice } \\
\text { pack. }\end{array}$ & Lab tech \\
\hline 62 & $\begin{array}{l}\text { Placing the plastic container of blood test tubes } \\
\text { in the Styrofoam box. Both the blood samples } \\
\text { and urine samples can be placed and } \\
\text { transported in the same Styrofoam box. }\end{array}$ & Lab tech \\
\hline 63 & $\begin{array}{l}\text { Affixing red colored sticker with print of } \\
\text { 'FRAGILE' on Styrofoam box. }\end{array}$ & Lab tech \\
\hline 64 & $\begin{array}{l}\text { Handing over the Styrofoam box to courier } \\
\text { services person. }\end{array}$ & Lab tech \\
\hline 65 & Collecting 15 dried DBS card in the zip lock & Lab tech \\
\hline 66 & $\begin{array}{l}\text { Putting the firmly closed zip lock in bubbled } \\
\text { envelop of Leopards courier for transporting to } \\
\text { SIUT Karachi }\end{array}$ & Lab tech \\
\hline 67 & $\begin{array}{l}\text { Placing yellow box for sharp disposal in the } \\
\text { large red waste bag. }\end{array}$ & Lab tech. \\
\hline 68 & $\begin{array}{l}\text { Incinerating or dispersing off large red waste } \\
\text { bag according to the local infectious medical }\end{array}$ & $\begin{array}{l}\text { Lab tech. \& } \\
\text { Medical Doctor }\end{array}$ \\
\hline
\end{tabular}




\begin{tabular}{l|l|l}
\hline \multicolumn{3}{|c}{ Steps to be followed in the field } \\
\hline Steps & Activity & Responsibility \\
\hline & waste management practice. & \\
\hline & $\begin{array}{l}\text { Items to be sent to Population Council, } \\
\text { Islamabad } \\
\text { OPD List } \\
\text { Log Sheet } \\
\text { Status Sheet } \\
\text { IDI Status Sheet } \\
\text { Questionnaire }\end{array}$ & Facilitator \\
\hline
\end{tabular}





\section{APPENDIX 9. MISCELLANEOUS: LETTER AND DOCUMENTS}

\section{Assurance Monitoring Check List}

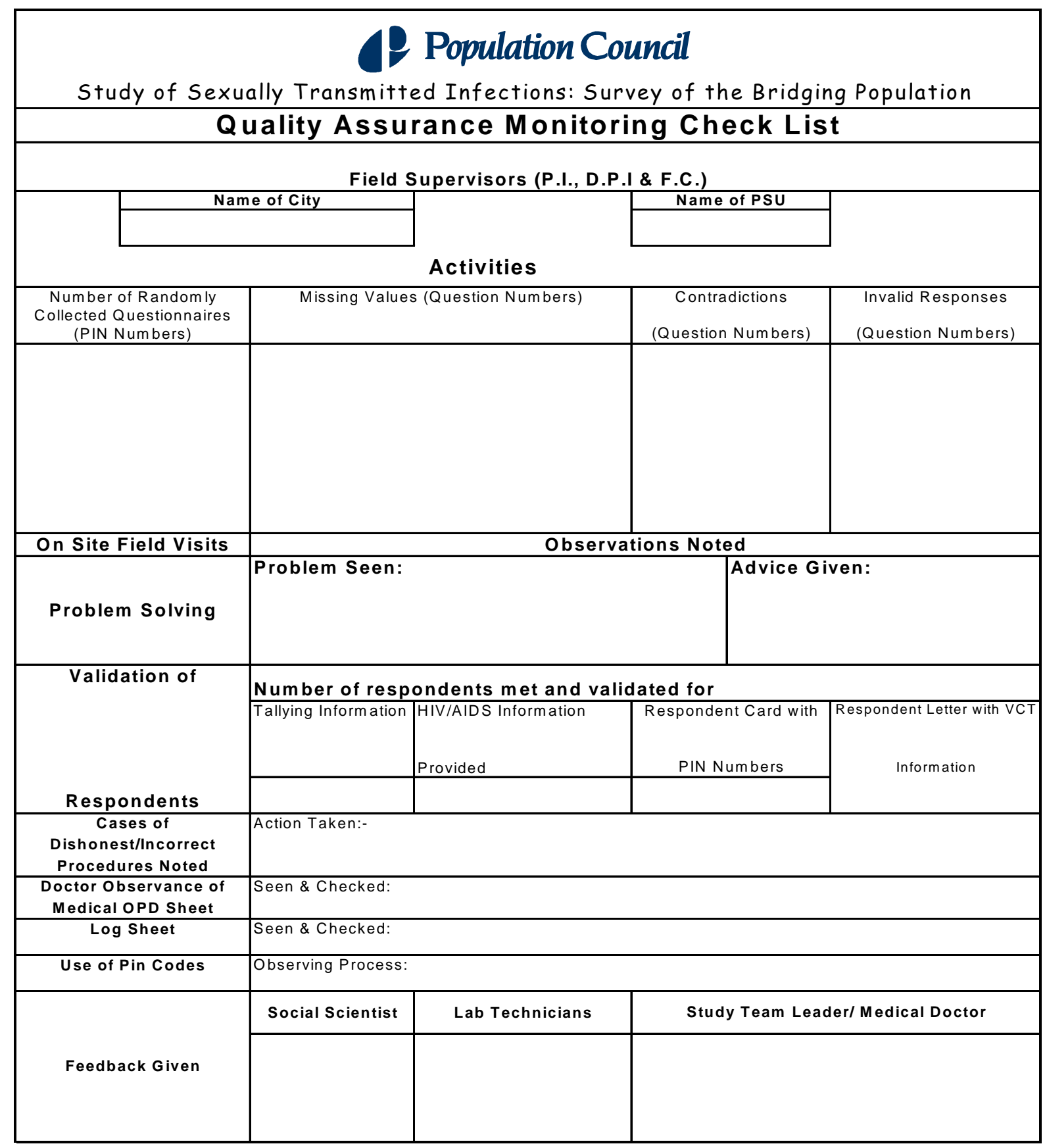




\section{Respondent's Letter}

\section{Dear Study Participants}

Thank you very much for your participation in the Study on Sexually Transmitted Infections: Survey of the Bridging Population.

Please find enclosed the addresses of Voluntary Counseling \& Testing Centers (VCTCs) located in __. You are requested to visit these centers where you can not only have yourself tested for HIV but also obtain other relevant information about the disease. You can also ask the question from the staff that is related to HIV/AIDS or other sexually transmitted diseases.

You can also call toll free number: 0800for information required on HIV/AIDS, during 9:00 am to 5:00 pm (Break 1:00 - 2:00 pm; Saturday and Sunday off).

After three weeks you are requested to contact Dr. on mobile No . He will let you know if the results of your blood tests have been received from Karachi. If they have been received, you will have to visit the Institute of . You will meet with Dr. and hand over to him you PIN Card, which has already been given to you. Based on the number on your card you will be handed over a sealed envelope that contains your test results. The envelope also contains special instructions on what you have to do in case the results are positive.

Thanking you once again for your cooperation!

\section{Yours sincerely,}

Population Council Study Team 
Study of SIs Among Urban Men in Pakistan

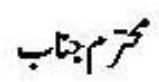

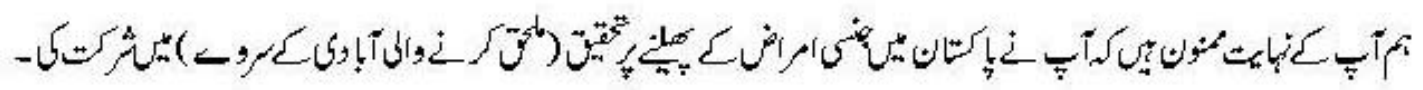

: 若

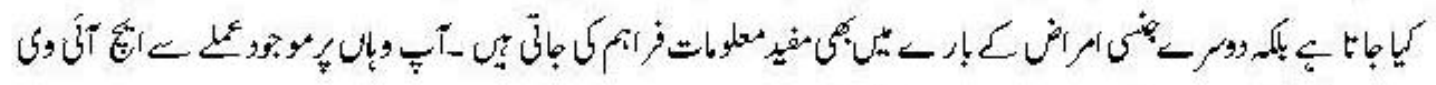
-

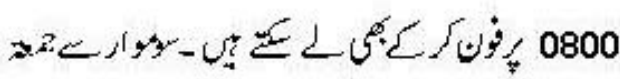

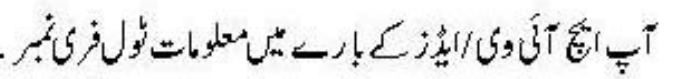

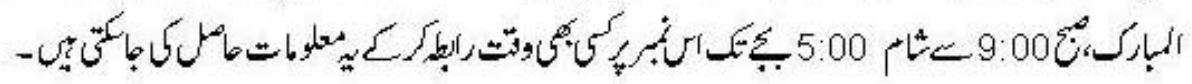

آب

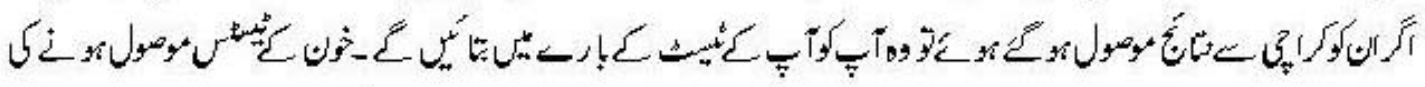
ك

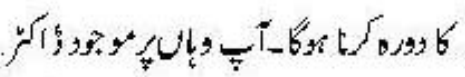

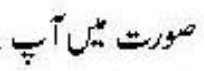

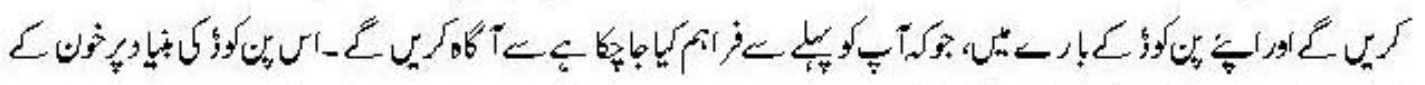

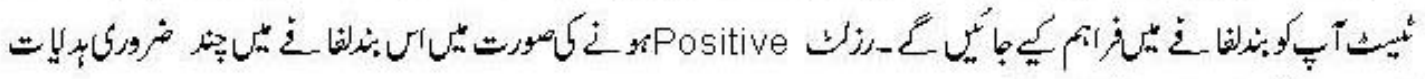

$-6$

:

والمالم

15

199 
Appendix

\section{Log Sheet for Lab Component}

\begin{tabular}{|c|c|c|c|c|c|c|c|c|c|}
\hline \multicolumn{10}{|c|}{ Log Sheet } \\
\hline \multicolumn{4}{|l|}{ City } & \multicolumn{3}{|l|}{ Date } & & & \\
\hline & & & & & & & & & \\
\hline S.No & Date & $\begin{array}{l}\text { Respondent } \\
\text { PIN Code }\end{array}$ & Field & Blood & Blood & Urine & $\begin{array}{l}\text { DBS- } \\
\text { Card }\end{array}$ & $\begin{array}{l}\text { Result } \\
\text { Syphilis }\end{array}$ & Comments \\
\hline & & & & $\begin{array}{l}\text { Purple - } \\
\text { top }\end{array}$ & Red-top & & & & If any \\
\hline 1 & & & & & & & & & \\
\hline 2 & & & & & & & & & \\
\hline 3 & & & & & & & & & \\
\hline 4 & & & & & & & & & \\
\hline 5 & & & & & & & & & \\
\hline 6 & & & & & & & & & \\
\hline 7 & & & & & & & & & \\
\hline 8 & & & & & & & & & \\
\hline 9 & & & & & & & & & \\
\hline 10 & & & & & & & & & \\
\hline 11 & & & & & & & & & \\
\hline 12 & & & & & & & & & \\
\hline & & & & & & & $\begin{array}{l}\text { Signat } \\
\text { Name }\end{array}$ & & \\
\hline & & & & & & & & & \\
\hline & & & & & & & & & \\
\hline & & & & & & & & & \\
\hline
\end{tabular}




\section{OPD Sheet for Medical Doctor}

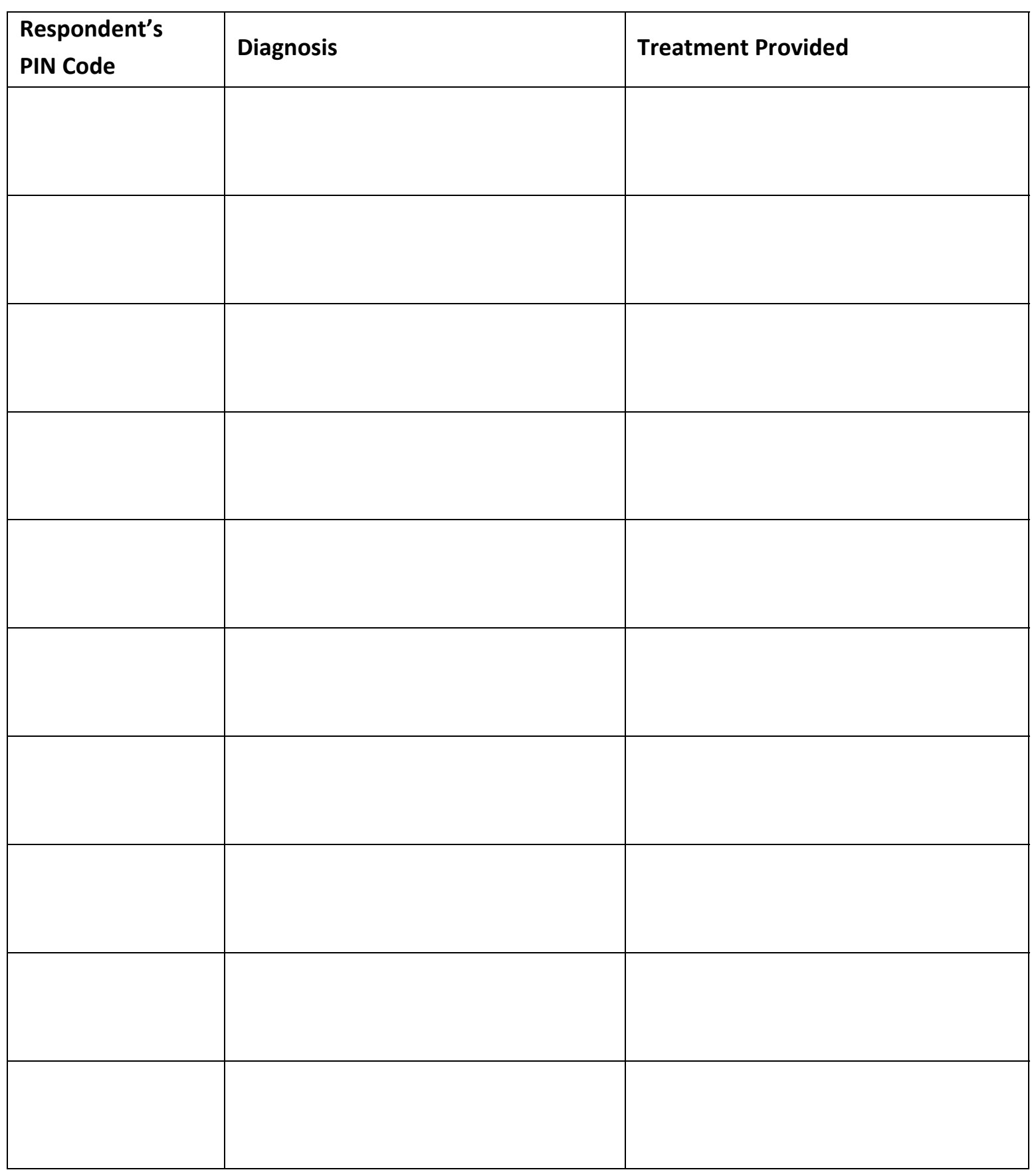


Appendix

\section{Referral Slips for Medical Doctors}

\section{Dear Doctor}

The Population Council is on behalf of the National AIDS Control Program, Ministry of Health,

Govt. of Pakistan conducting a Study of Sexually Transmitted Infections: Survey of the Bridging Population.

During this study we have identified Mr. who needs immediate medical care. I am referring him to you for further expert medical attention.

Thanking you 


\title{
APPENDIX 10. BEHAVIORAL QUESTIONNAIRE
}

Serial Number:_L__L_

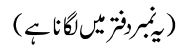

\section{Study of Sexually Transmitted Infections:}

\author{
Survey of The \\ Bridging Population
}

(Urban Men Ages 16-45)

Strictly Confidential

This information is confidential and the name and address of the respondent will not be used for any other purpose than the "Sexually Transmitted Infections Survey" of the Population Council.

$$
\text { صينحران }
$$

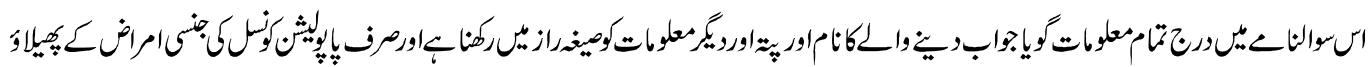

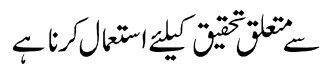

\section{QP Population Council}


Serial Number of questionnaire $\quad \ldots+\left.\left.{ }_{--}\right|_{--}\right|_{-}$

Study of Sexually Transmitted Infections (STIs)

List all male members of household who have passed last night in this household

\begin{tabular}{|c|c|c|c|c|c|c|c|c|}
\hline R1 & $\mathrm{R} 2$ & R3 & $\mathrm{R} 4$ & R5 & & & & \\
\hline \multirow[t]{2}{*}{$\begin{array}{l}\text { Sr. } \\
\text { No }\end{array}$} & \multirow[t]{2}{*}{$\begin{array}{c}\text { Name of male } \\
\text { members }\end{array}$} & \multirow{2}{*}{$\begin{array}{l}\text { Age in } \\
\text { complete } \\
\text { d years }\end{array}$} & \multirow{2}{*}{$\begin{array}{c}\text { Education } \\
\text { (schooling grades } \\
\text { passed) }\end{array}$} & \multirow[t]{2}{*}{ Profession } & \multicolumn{2}{|c|}{$\begin{array}{l}\text { Eligible for } \\
\text { interview }\end{array}$} & \multicolumn{2}{|c|}{$\begin{array}{l}\text { Selected for } \\
\text { interview }\end{array}$} \\
\hline & & & & & Yes & No & Yes & No \\
\hline 1 & & & $-I_{-}$ & & 1 & & 1 & \\
\hline 2 & & & $-1--$ & & 1 & & 1 & \\
\hline 3 & & & $-I_{--}$ & & 1 & & 1 & \\
\hline 4 & & & $-I_{--}$ & & 1 & & 1 & \\
\hline 5 & & & $-I_{--}$ & & 1 & & 1 & \\
\hline 6 & & & --1 & & 1 & & 1 & \\
\hline 7 & & & $-1-$ & & 1 & & 1 & \\
\hline 8 & & & $-I_{--}$ & & 1 & & 1 & \\
\hline 9 & & & $-I_{-}$ & & 1 & & 1 & \\
\hline 10 & & & $-1-$ & & 1 & & 1 & \\
\hline 11 & & & --1 & & 1 & & 1 & \\
\hline 12 & & & $-I_{--}$ & & 1 & & 1 & \\
\hline 13 & & & $-I_{-}$ & & 1 & & 1 & \\
\hline 14 & & & $-1-$ & & 1 & & 1 & \\
\hline 15 & & & $-I_{-}$ & & 1 & & 1 & \\
\hline
\end{tabular}

Use additional sheet if needed

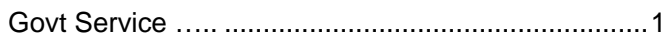

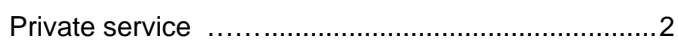

Teaching in government school .......................... 3

Teaching in private school ................................ 4

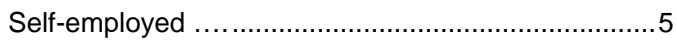

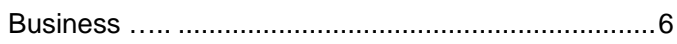

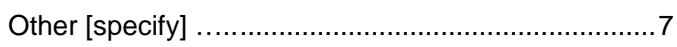




\section{CONTENTS OF THE QUESTIONNAIRE}

Section

Section - A

Section - B

Section - C

Section - D

Section - E

Section - F

Section - G

Section - H

Section - I
Title

Page

Informed consent form and identification

03

Socio-demographic characteristics of respondent

Information about wife and household decision making

General health

Sexual behavior, condom use, and personal hygiene

Blood transfusion, injection taking, and drug use history

Knowledge about STIs and treatment seeking behavior

Personal experiences with STIs

36

Sexual behavior and attitude 


\section{Section - A: Informed Consent and Identification \\ Consent statement for behavioral component \\ Introduction:}

[Please read the statement below out loud before continuing with the questionnaire]

Assalamm-o-Alaikum. My name is . I am working with an organization called the Population Council that is helping the government of Pakistan to design health programs in Pakistan. We are interviewing men (single and married) living in urban areas either with or away from their families. In order to improve health services for men living in urban areas we need to know more about the health problems faced by men in particular. I am here today to talk with you and to gather information about your health and illnesses.

I am going to ask you some very personal questions that some people find difficult to answer. Your answers are completely confidential. Your name will not be written on this form and will never be used in connection with any of the information you tell me. You don't have to answer any questions that you don't want to answer, and you may end this interview at any time you want to. However, your honest answers to these questions will help us better understand what people think, say, and do about certain kinds of behaviors. We will also be asking you to voluntarily provide blood and urine samples for the testing of common and important sexually transmitted infections, which are HSV-2, syphilis, HIV, Gonorrhea, and Chlamydia. A medical doctor who is part of our team is stationed nearby and shall be available to provide you with any advice for any medical problem that you may be facing. Your participation is purely voluntary and you shall not be compensated in any other way.

We would greatly appreciate your help in responding to this survey. It will take thirty to forty minutes to ask the questions. Are you willing to answer these questions?

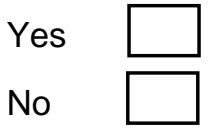

From every respondent who said they had paid for sex in the last 03 months the following additional consent will be obtained:

If you wish we would like to seek some further information on the same questions we have asked you before. The process will take about 40 minutes. Your participation is voluntary and there is no penalty for refusing to take part. Your answers will be kept completely confidential. You may refuse to answer any question in the interview or to stop the interview at any time.

Informed Consent Statement for obtaining Biological specimens: The Population Council, Pakistan is an international research organization. The Population Council is undertaking a research study for the Government of Pakistan. The results of the study will help in developing better health services for the population in general and men in particular. We would like to request you to voluntarily give us a sample of your blood and urine. The blood sample will be taken from your vein in your arm, and a syringe will withdraw not more than $8 \mathrm{ml}$ of blood (approximately 1 tablespoon). Every care will be made to ensure that you do not endure any pain, discomfort, injury, or inadvertent infection during the process. In case of any injury or discomfort arising as a result of the procedure, immediate essential short-term medical care and treatment as determined by the study team doctor will be made available without charge. You have the right to decline from giving the samples. 


\section{Informed Consent Statement for Parents/Guardian of young adults aged 16 - 17 years}

Assalamm-o-Alaikum. My name is I am working with an organization called the Population Council that is helping the government of Pakistan to design health programs in Pakistan. We are interviewing men (single and married) living in urban areas either with or away from their families. In order to improve health services for men living in urban areas we need to know more about the health problems faced by men in particular. I am here today to talk to your son/ward and to gather information about his health and illnesses.

I am going to ask your son some very personal questions that some people find difficult to answer. The answers are completely confidential. Your son's name will not be written on this form and will never be used in connection with any of the information provided by him. He does not have to answer any questions that he doesn't want to and he may end this interview at any time he wants to. However, his honest answers to these questions will help us better understand what people think, say, and do about certain kinds of behaviors. We will also be asking your son to voluntarily provide blood and urine samples for the testing of important and common sexually transmitted infections. A medical doctor who is part of our team is stationed nearby and shall be available to provide your son with any advice for any medical problem that he may be facing. His participation is purely voluntary and he shall not be compensated in any other way.

We would greatly appreciate his help in responding to this survey. It will take thirty to forty minutes to ask the questions.

If you agree to let your son participate, the interviewer will meet with your son and ask him certain questions.

A further component of this study includes obtaining specimens of blood and urine.

We would like to request you to permit your son to voluntarily give us a sample of his blood and urine. The blood sample will be taken from his vein in his arm, and a syringe will withdraw not more than $8 \mathrm{ml}$ of blood (approximately 1 tablespoon). Every care will be made to ensure that he does not endure any pain, discomfort, injury, or inadvertent infection during the process. In case of any injury or discomfort arising as a result of the procedure, immediate essential short-term medical care and treatment as determined by the study team doctor will be made available without charge. Your son has the right to decline from giving the samples. The results of the investigations will remain anonymous and cannot be identified by his name. A personal identification number that will be known only to me will mark the samples. The results can be collected from a designated centre. The information obtained from the results, as part of the study will be transmitted only in a form that cannot be identified by your son's name. 
Do you agree to have the interviewer talk with your son/relative?

$\begin{array}{ll}\text { Yes } & \square \\ \text { No } & \square\end{array}$

Do you agree to let your son/relative give a sample of his blood/urine?

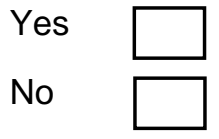

Also, if you agree to participate, one of us will conduct a more in-depth interview with your son/relative to ask more detailed questions. The interview could be done at your home or at any other place that we can agree upon. The interview will be private and the arrangements for the detailed interview will be confidential.

Do you agree to have a detailed in-depth interview with your son?

Yes

No

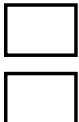

Please print clearly:

Signature or thumb impression of respondent

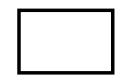

OR

Tape recorded consent

OR

(Signature of interviewer)

Verbal consent verified by witness

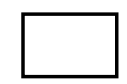

(Signature of interviewer)

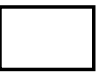

(Signature of witness)

(Interviewer's name)

(Signature of Interviewer) 
Subject Statement:

\begin{tabular}{|c|c|}
\hline $\begin{array}{l}\text { A1 } \\
\text { Do you have questions you would like to discuss at this point? }\end{array}$ & 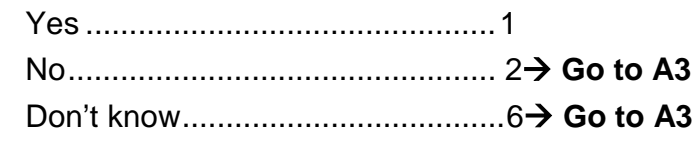 \\
\hline $\begin{array}{l}\text { A2 } \\
\text { [IF YES] what are the questions? }\end{array}$ & [Specify] \\
\hline A3.May I start the interview? & $\begin{array}{l}\text { Yes } \\
\text { [If No, then complete Identification page and } \\
\text { end interview] }\end{array}$ \\
\hline
\end{tabular}

\section{A4}

"I have been given an opportunity to ask any questions I may have, and all such questions or inquiries have been answered to my satisfaction."

"I further understand that my records will be kept confidential and that I may withdraw from this study at any time."

I voluntarily agree to give without any coercion or assurance of compensation, samples of my blood and urine for testing three common and important sexually transmitted infections. I understand that the results of the investigations will remain anonymous and cannot be identified by my name. A personal identification number that will be known only to me will mark the samples. I can collect the results from the designated centre. I also understand that the information obtained from my results as part of the study will be transmitted only in a form that cannot be identified with me.

"If I require medical treatment as a result of physical injury arising from my participation in this study, immediate, essential, short-term medical care and treatment as determined by the doctors in this study will be made available without charge to me. I will receive no monetary compensation for any other care, but medical consultation and appropriate referral services are available. Further information on the availability of medical care and treatment for any physical injury resulting from my participation in this study may be obtained from the Investigator."

"My withdrawals from this study or my refusal to participate will in no way affect my medical care from the hospital or clinic."

"I have been informed orally and in writing of whom to contact in case of an emergency. I agree to participate in this study as a volunteer subject."

Signature or thumb impression of respondent

OR

Tape recorded consent

(Signature of interviewer)

OR

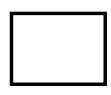

Verbal consent verified by witness

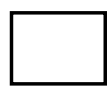

(Signature of witness)

(Interviewer's name)

(Signature of Interviewer) 


\section{Identification of Area and Respondent}

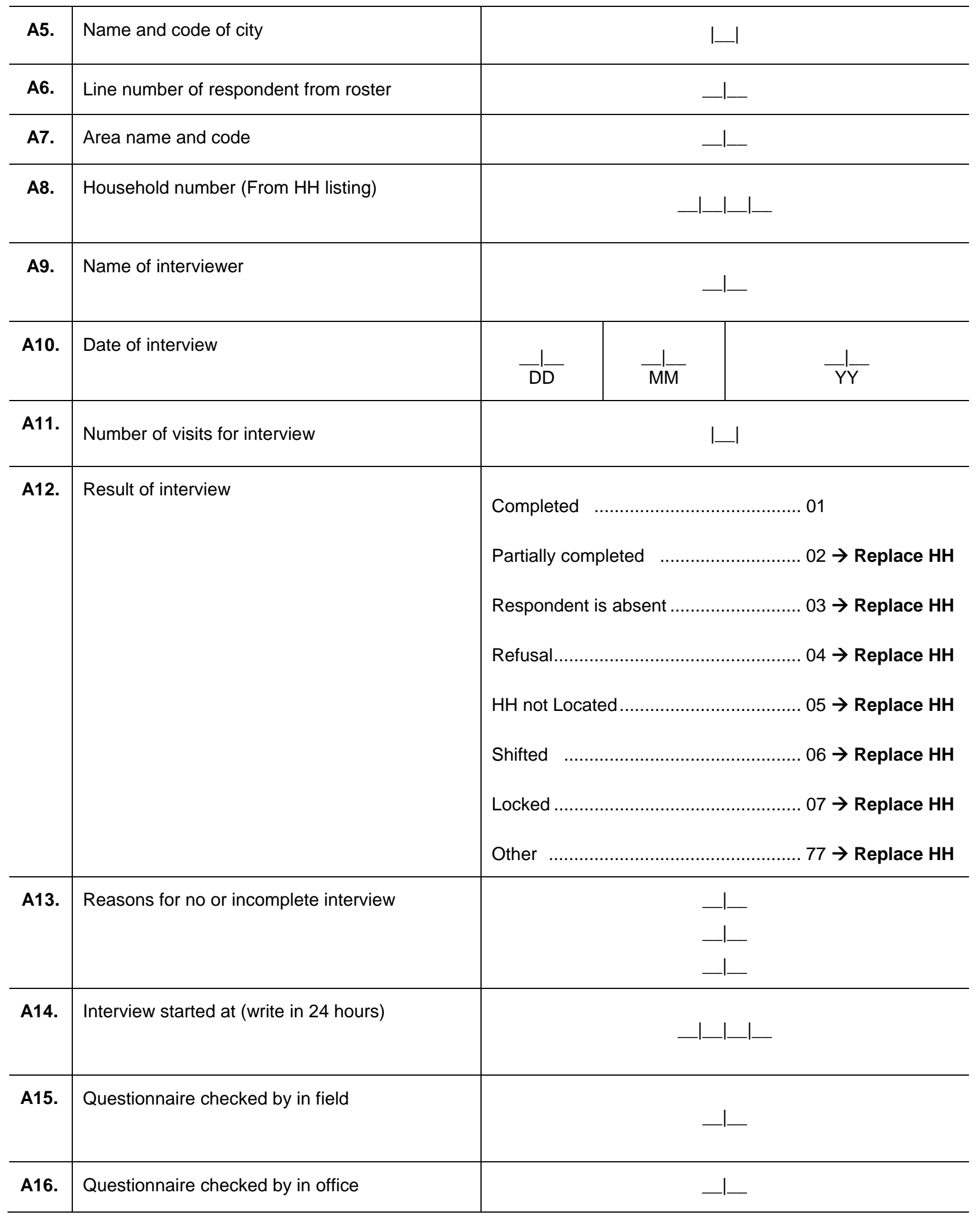




\begin{tabular}{|c|c|c|}
\hline A17. & Questionnaire entered by & -1 \\
\hline \multicolumn{3}{|c|}{ Information About Blood and Urine Sample } \\
\hline A18. & Has respondent given blood sample? & Yes. \\
\hline A19. & Has respondent given urine sample? & 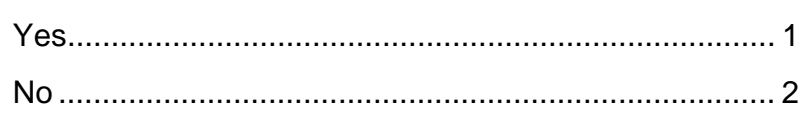 \\
\hline
\end{tabular}

\section{If $\mathrm{A} 18=2 \& \mathrm{~A} 19=2 \rightarrow$ Go to $\mathrm{B} 1$}

\begin{tabular}{l|l|l}
\hline A20. & Please affix pin code here & . \\
& Please write down the pin code & \\
\hline
\end{tabular}


Section - B: Socio-Demographic Characteristics of Respondents

\begin{tabular}{|c|c|c|}
\hline \multicolumn{2}{|r|}{ Questions and Filters } & Responses and Codes \\
\hline B1. & How old are you? & Age in completed years ................................. \\
\hline B2. & In which month, year were you born? & 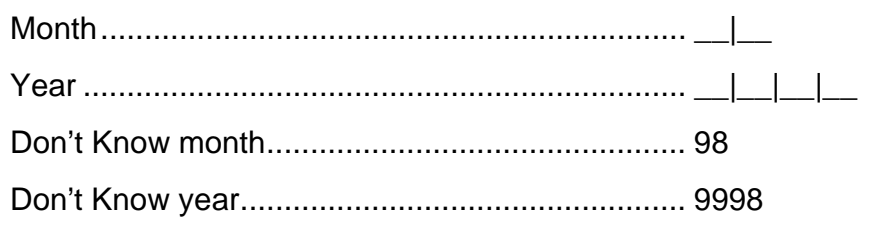 \\
\hline B3. & $\begin{array}{l}\text { How many years of education have you } \\
\text { completed? }\end{array}$ & 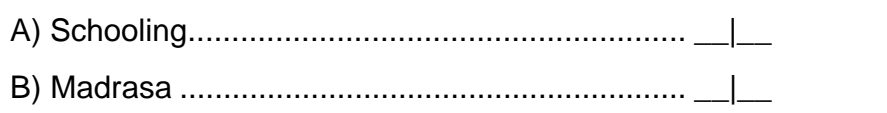 \\
\hline B4. & $\begin{array}{l}\text { What is the highest degree/diploma that you } \\
\text { have obtained? } \\
\text { [Interviewer] Ask from those who have } \\
\text { completed } 10 \text { years of schooling. }\end{array}$ & 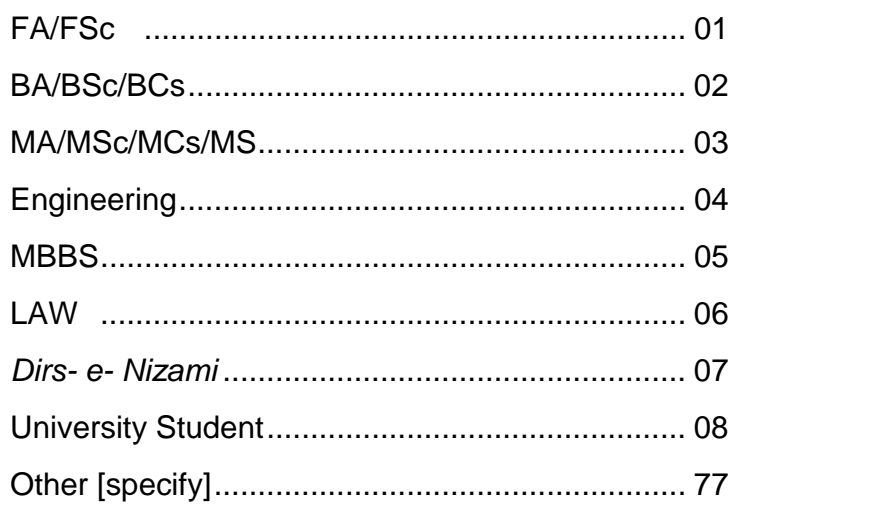 \\
\hline B5 & What is your mother tongue (language)? & Punjabi \\
\hline B6. & Have you ever been married? & Yes \\
\hline B7 & $\begin{array}{l}\text { Are you now married, single, widower, } \\
\text { seperated or divorced? }\end{array}$ & 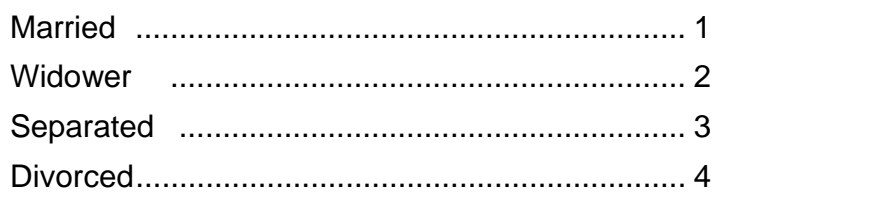 \\
\hline B8. & How many living children do you have? & a) Total \\
\hline
\end{tabular}




\begin{tabular}{|c|c|c|}
\hline B9. & $\begin{array}{l}\text { Do you live with parents, wife, parents and wife, } \\
\text { alone with relatives, with friends or in a hostel? }\end{array}$ & $\begin{array}{l}\text { Parents } \\
\text { Parents and wife } \\
\text { Wife } \\
\text { Alone } \\
\text { Relative } \\
\text { Friends }\end{array}$ \\
\hline B10 & $\begin{array}{l}\text { For how long have you been living away from } \\
\text { your natal or marital home? }\end{array}$ & a) Months \\
\hline B11 & $\begin{array}{l}\text { Are you living in your own house, rented house, } \\
\text { or hostel? }\end{array}$ & $\begin{array}{l}\text { Alone in own house } \\
\text { Alone in rented house } \\
\text { Alone in room/hostel/guesthouse } \\
\text { Share rented house } \\
\text { Share rented room } \\
\text { Shared room in a hostel }\end{array}$ \\
\hline B12 & $\begin{array}{l}\text { If living alone in a shared house / hostel, do you } \\
\text { live alone in the room or share it? }\end{array}$ & Alone \\
\hline B13 & $\begin{array}{l}\text { How many persons sleep in the same room with } \\
\text { you? }\end{array}$ & Number of persons \\
\hline B14 & Where is your family/parents home? & Name of the village/ city/ district \\
\hline B15 & $\begin{array}{l}\text { What was the reason that you have left your } \\
\text { native (original) city? } \\
\text { (Multiple responses allowed) }\end{array}$ & $\begin{array}{l}\text { Family Reasons } \\
\text { Business/Employment } \\
\text { Education } \\
\text { Other [specify] }\end{array}$ \\
\hline B16 & $\begin{array}{l}\text { How often do you visit your natal or marital } \\
\text { home? }\end{array}$ & After a month \\
\hline
\end{tabular}




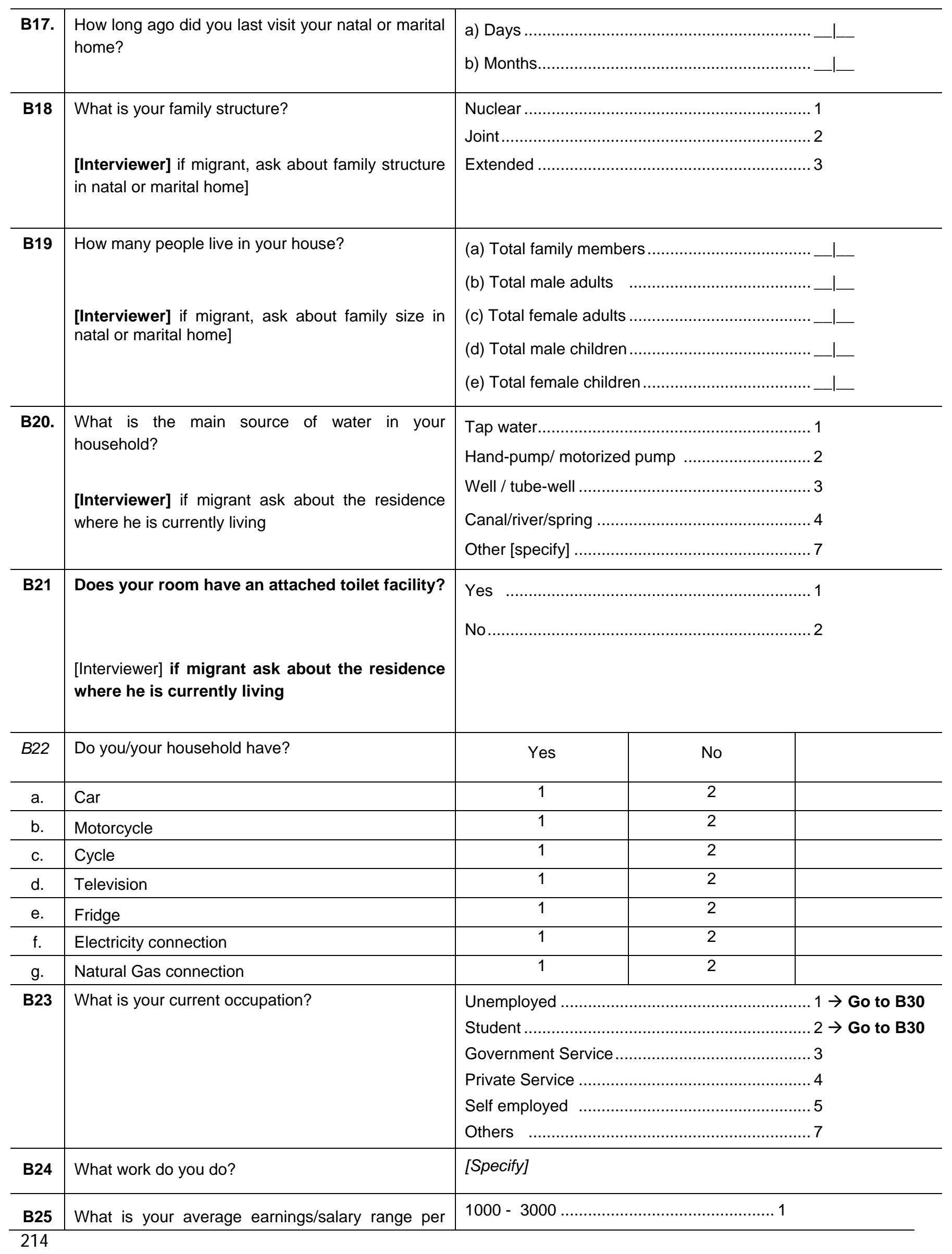




\begin{tabular}{|c|c|c|}
\hline & month? & 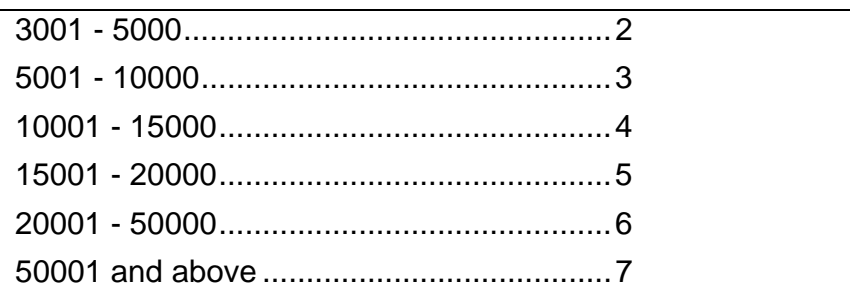 \\
\hline B26 & $\begin{array}{l}\text { For how long have you been working in your } \\
\text { current occupation? }\end{array}$ & a) Months \\
\hline B27 & $\begin{array}{l}\text { As part of your job/work/business do you have to } \\
\text { travel outside this city? }\end{array}$ & Yes \\
\hline B28 & $\begin{array}{l}\text { As part of your job/work/business are you required } \\
\text { to stay outside your home over night within this } \\
\text { city? }\end{array}$ & Yes \\
\hline B29. & $\begin{array}{l}\text { How many nights per month do you have to stay } \\
\text { out of your (current) home as part of your } \\
\text { job/work/business? }\end{array}$ & Number of nights \\
\hline B30. & $\begin{array}{l}\text { In last } 12 \text { months have you been away from your } \\
\text { current place of residence for over night or more } \\
\text { time continuously for leisure? }\end{array}$ & No \\
\hline B31. & $\begin{array}{l}\text { Were you away alone or accompanied by your } \\
\text { family on this leisure trip? }\end{array}$ & 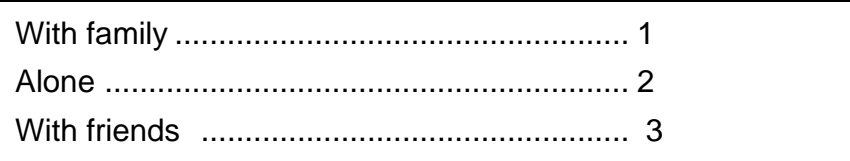 \\
\hline B32 & $\begin{array}{l}\text { How many days (collectively) were you away in the } \\
\text { last } 12 \text { months? }\end{array}$ & Number of days …………………………….....___ \\
\hline B33. & $\begin{array}{l}\text { What was the reason that you had been away from } \\
\text { home? } \\
\text { (Multiple responses allowed) }\end{array}$ & 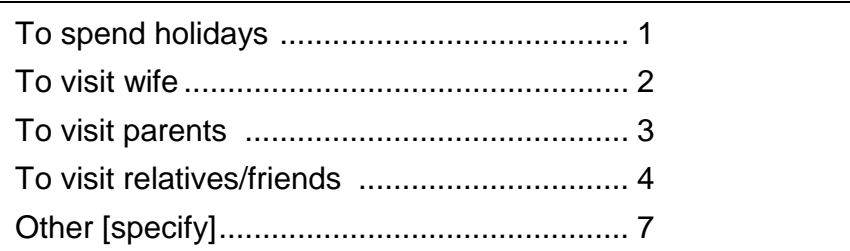 \\
\hline B34. & Have you ever been to any foreign country? & Yes \\
\hline
\end{tabular}




\begin{tabular}{|c|c|c|}
\hline B35 & B36 & B37 \\
\hline $\begin{array}{l}\text { Specify names of countries that you } \\
\text { have visited in last ten years? } \\
\text { [Interviewer] please start from the } \\
\text { country visited most recently. }\end{array}$ & $\begin{array}{l}\text { How many months ago did you } \\
\text { visit each country? }\end{array}$ & $\begin{array}{l}\text { For what purpose did you visit a } \\
\text { foreign country? } \\
\text { Business } \\
\text { To spend holidays } \\
\text { Religious rituals } \\
\text { Other [specify] }\end{array}$ \\
\hline $\begin{array}{l}\text { a) } \\
\text { b) } \\
\text { c) } \\
\text { d) } \\
\text { e) }\end{array}$ & $\begin{array}{l}-1 \\
-1 \\
-1 \\
-1 \\
-1\end{array}$ & $\begin{array}{l}-1 \\
-1 \\
-1 \\
-1 \\
-1\end{array}$ \\
\hline
\end{tabular}




\section{Section - C: Information About Wife And Household Decision Making}

\begin{tabular}{|c|c|c|}
\hline \multicolumn{2}{|c|}{ Questions and Filters } & Responses and Codes \\
\hline C1. & $\begin{array}{l}\text { What is current marital status of respondent? } \\
\{\text { Check with B7\} } \\
\text { [If separated, divorced or widowed ask about ex- } \\
\text { wife] }\end{array}$ & 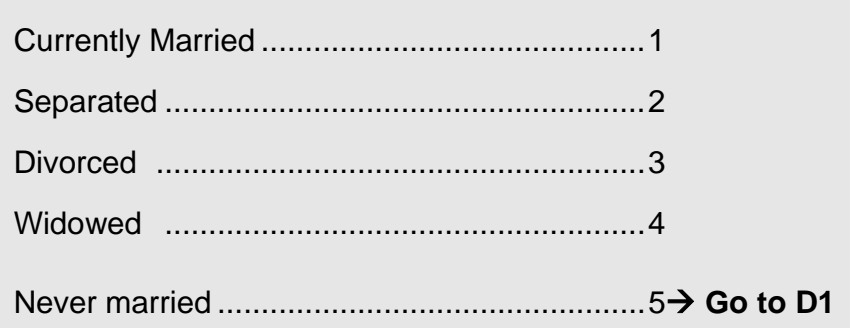 \\
\hline $\mathbf{C 2}$. & Have you been married once or more than once? & Once \\
\hline C3. & How many marriages have you had? & Number of marriages \\
\hline C4. & How old were you when you got married (first time)? & Age in years \\
\hline C5. & $\begin{array}{l}\text { What was the age of your (first) wife at the time of } \\
\text { marriage? }\end{array}$ & Age in years \\
\hline
\end{tabular}

Now I would like to ask you some questions about your current marriage/ wife

\begin{tabular}{|c|c|c|}
\hline C6. & $\begin{array}{l}\text { How many schooling grades has your wife } \\
\text { completed? }\end{array}$ & 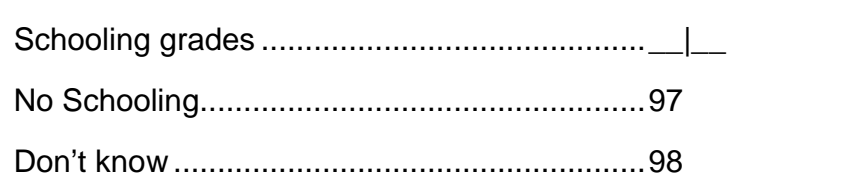 \\
\hline C7. & Has your wife been married more than once? & Yes \\
\hline C8. & Is your wife employed? & Yes \\
\hline c9. & Where does she work? & Govt Service \\
\hline
\end{tabular}




\begin{tabular}{|c|c|c|}
\hline \multicolumn{2}{|c|}{ Questions and Filters } & \multirow[b]{2}{*}{ 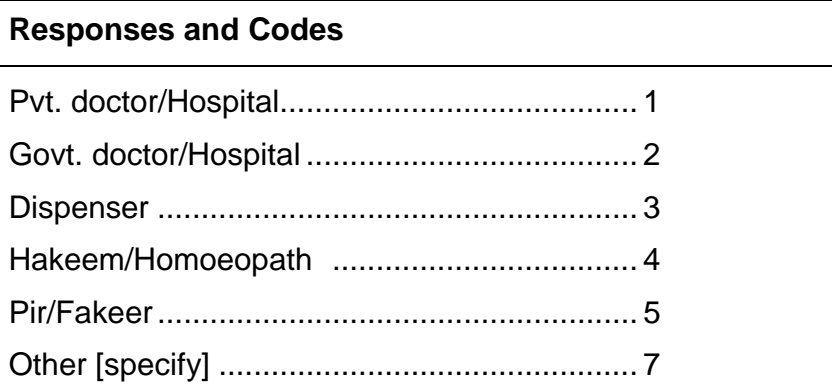 } \\
\hline C10. & $\begin{array}{l}\text { Where does your wife usually go for treatment if she } \\
\text { has a health complaint? }\end{array}$ & \\
\hline C11. & $\begin{array}{l}\text { Who makes the major decisions about health care } \\
\text { for your wife in your family/household? }\end{array}$ & Wife \\
\hline C12. & $\begin{array}{l}\text { Who makes the major decisions regarding large } \\
\text { household purchases in your family/household? }\end{array}$ & Myself \\
\hline C13 & $\begin{array}{l}\text { Do you and your wife discuss issues related to child } \\
\text { spacing? }\end{array}$ & 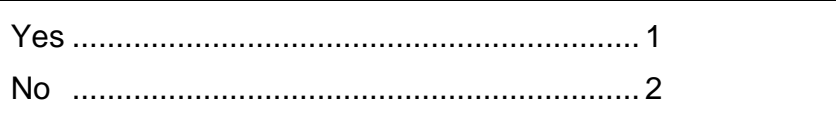 \\
\hline C14 & $\begin{array}{l}\text { Have you or your wife ever used any method of } \\
\text { family planning? }\end{array}$ & Yes \\
\hline C15 & $\begin{array}{l}\text { What methods of family planning have you or your } \\
\text { wife ever used? } \\
\text { (Multiple responses are allowed) }\end{array}$ & $\begin{array}{l}\text { Oral Pills } \\
\text { Condom } \\
\text { IUCD } \\
\text { Injectable } \\
\text { Norplant } \\
\text { Female Sterilization } \\
\text { Male Sterilization } \\
\text { Rithdrawal }\end{array}$ \\
\hline
\end{tabular}




\begin{tabular}{|c|c|c|}
\hline \multicolumn{2}{|c|}{ Questions and Filters } & Responses and Codes \\
\hline C16 & $\begin{array}{l}\text { Are you or your wife currently using any method of } \\
\text { family planning? }\end{array}$ & Yes \\
\hline C17 & $\begin{array}{l}\text { What method of family planning are you or your wife } \\
\text { currently using? }\end{array}$ & Oral Pills \\
\hline
\end{tabular}


Section D: General Health

(Now I will ask some questions about your general health)

\begin{tabular}{|c|c|c|c|c|}
\hline & Questions and Filters & \multicolumn{3}{|c|}{ Responses and Codes } \\
\hline D1. & $\begin{array}{l}\text { How would you describe your health in general- } \\
\text { healthy, unhealthy, or in between? }\end{array}$ & \multicolumn{3}{|c|}{ 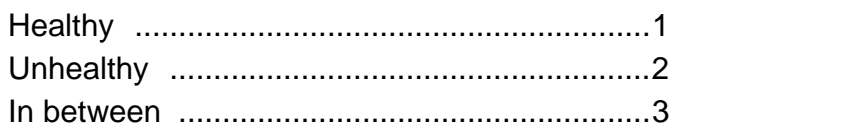 } \\
\hline D2. & Have you ever had surgery in your life? & \multicolumn{3}{|c|}{ 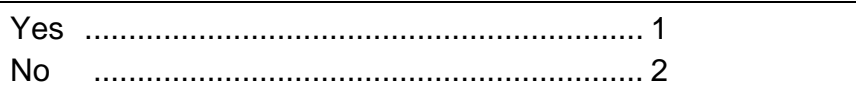 } \\
\hline D3. & $\begin{array}{l}\text { Do you have any long-term (chronic) health } \\
\text { problem? }\end{array}$ & \multicolumn{3}{|c|}{ Yes } \\
\hline D4. & $\begin{array}{l}\text { If yes, what type of long-term (chronic) } \\
\text { health problem do you have? }\end{array}$ & \multicolumn{3}{|l|}{ (Specify) } \\
\hline D5. & $\begin{array}{l}\text { Have you had any illness in the last } 3 \\
\text { months? }\end{array}$ & \multicolumn{3}{|c|}{ Yes No } \\
\hline D6. & $\begin{array}{l}\text { If yes, what type of illness have you most } \\
\text { recently had? }\end{array}$ & \multicolumn{3}{|c|}{ 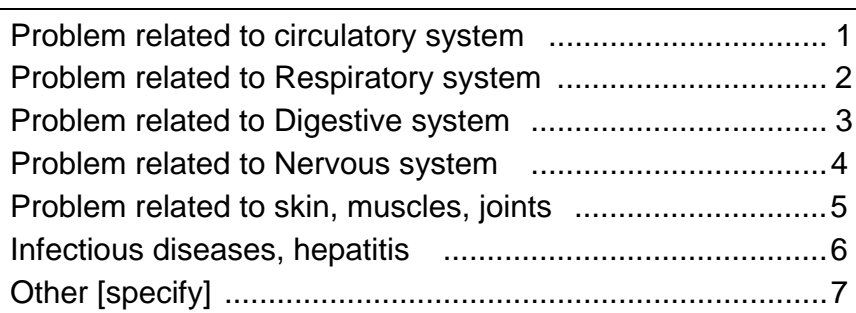 } \\
\hline D7. & Did you seek treatment for your last illness? & \multicolumn{3}{|c|}{ Yes } \\
\hline \multirow{2}{*}{ D8. } & \multirow[t]{2}{*}{ From whom did you seek treatment? } & \multicolumn{2}{|c|}{1} & 2 \\
\hline & & Yes & No & Order \\
\hline a. & Doctor & 1 & 2 & {$[\quad]$} \\
\hline b. & Dispenser/paramedic & 1 & 2 & {$[\quad]$} \\
\hline c. & Hakeem & 1 & 2 & {$[\quad]$} \\
\hline d. & Quack & 1 & 2 & [ ] \\
\hline e. & Homeopath & 1 & 2 & [ ] \\
\hline f. & Pir Fakeer & 1 & 2 & [ ] \\
\hline g. & Self Medication & 1 & 2 & [ ] \\
\hline h. & Home Remedy & 1 & 2 & [ ] \\
\hline i. & Other (specify) & 1 & 2 & [ ] \\
\hline
\end{tabular}




\section{Section - E: Sexual Behavior, Condom Use and Personal Hygiene}

Now I am about to ask you some personal questions that some people find difficult to answer. Your answers are completely confidential. You do not have to answer any question that you do not want to answer, and you may end this interview at any time you want to. However your honest answers to these questions will help us better understand what people think, say, and do about certain kinds of behaviors related to men's health. We would greatly appreciate your help in responding to these questions.

\begin{tabular}{|c|c|c|}
\hline \multicolumn{2}{|c|}{ Questions and Filters } & Responses and Codes \\
\hline E1. & $\begin{array}{l}\text { Many males by the time they are your age become } \\
\text { sexually active, some have had sexual relations. Have } \\
\text { you ever had sexual intercourse? } \\
\text { [Ask from only unmarried men] }\end{array}$ & Yes \\
\hline E2. & $\begin{array}{l}\text { With whom did you have sexual intercourse for the } \\
\text { first time? }\end{array}$ & $\begin{array}{l}\text { Wife } \\
\text { FCSW } \\
\text { MCSW } \\
\text { Male } \\
\text { Female/friend }\end{array}$ \\
\hline E3. & $\begin{array}{l}\text { At what age, did you have sexual intercourse for } \\
\text { the first time? }\end{array}$ & Years \\
\hline E4. & $\begin{array}{l}\text { How old was the person with whom you had your first } \\
\text { sexual intercourse? }\end{array}$ & Years \\
\hline E5. & $\begin{array}{l}\text { How long ago did you have sexual intercourse the last } \\
\text { time? }\end{array}$ & 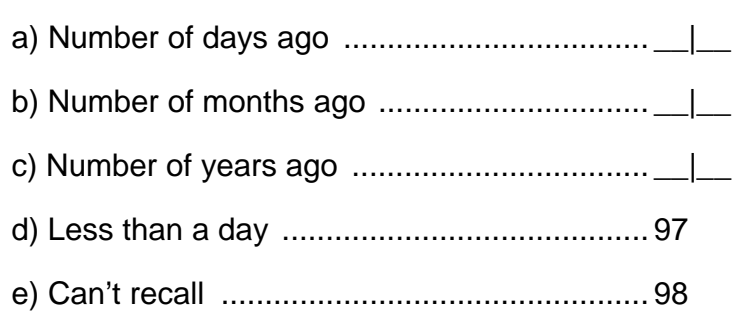 \\
\hline
\end{tabular}


Now I am going to ask you a few questions that are related to other than your wife.

[Interviewer] Please ask E6 of this section by row wise and all other questions of this column wise.

\begin{tabular}{|c|c|c|c|c|c|c|}
\hline \multicolumn{2}{|r|}{ Question and Filters } & $\begin{array}{c}\text { A - Female } \\
\text { (Other than FSW) } \\
\text { (Prompt) }\end{array}$ & $\begin{array}{c}\text { B - Female sex worker } \\
\text { (Prompt) }\end{array}$ & $\begin{array}{c}\text { C - Male sex worker } \\
\text { (Prompt) }\end{array}$ & $\begin{array}{c}\text { D - Male } \\
\text { (Other than MSW) } \\
\text { (Prompt) }\end{array}$ & $\begin{array}{l}\text { E - Hijra } \\
\text { (Prompt) }\end{array}$ \\
\hline E6. & $\begin{array}{l}\begin{array}{l}\text { Have you ever had } \\
\text { sexual } \\
\text { with.....? }\end{array} \\
\text { intercourse }\end{array}$ & 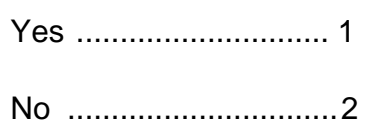 & 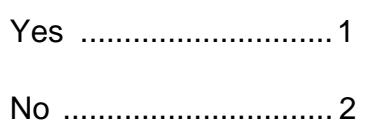 & 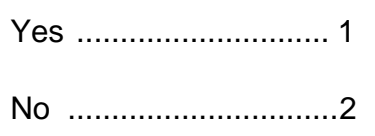 & 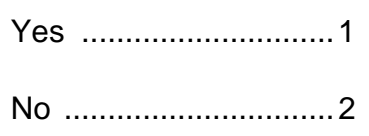 & 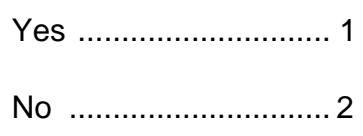 \\
\hline E7. & $\begin{array}{l}\text { How many [ }] \text { sexual } \\
\text { partners have you had } \\
\text { during your life time? }\end{array}$ & $\begin{array}{l}\# \text { of partners ......... } \_- \\
\text {Can't recall .......... } 98\end{array}$ & $\begin{array}{l}\# \text { of partners ........ }-1 \text { - } \\
\text { Can't recall...........98 }\end{array}$ & $\begin{array}{l}\# \text { of partners ........ } \_- \\
\text {Can't recall .......... } 98\end{array}$ & $\begin{array}{l}\# \text { of partners ......... }- \\
\text { Can't recall............98 }\end{array}$ & $\begin{array}{l}\text { \# of partners ........ _ _ } \\
\text { Can't recall .......... } 98\end{array}$ \\
\hline E8. & $\begin{array}{l}\text { How many [ }] \text { sexual } \\
\text { partners have you had } \\
\text { in last } 12 \text { months? }\end{array}$ & \# of partners .........__ _ & \# of partners ........ _ _ & \# of partners ......... - _ & \# of partners ........ - _ & \# of partners-- __ _ _ \\
\hline E9. & $\begin{array}{l}\text { How many [ }] \text { sexual } \\
\text { partners have you had } \\
\text { in the last } 3 \text { months? }\end{array}$ & \# of partners .........__ _ & \# of partners ........ _ _ & \# of partners ......... - _ & \# of partners ........ _ _ & \# of partners--__ _ _ \\
\hline E10. & $\begin{array}{l}\text { How many times did } \\
\text { you have sex with } \\
{[\quad] \text { in last } 3 \text { months? }}\end{array}$ & \# of times .............. & \# of times ............. & \# of times.............. & \# of times ............. & \# of times ....... _ _ \\
\hline E11. & $\begin{array}{l}\text { How long ago did you } \\
\text { last have intercourse } \\
\text { with }[\quad] \text { ? }\end{array}$ & \# of days .............. - & \# of days .............. _ _ & \# of days................ & \# of days ............... _ _ & $\#$ of days .............. - \\
\hline
\end{tabular}




\begin{tabular}{|c|c|c|c|c|c|c|}
\hline & Question and Filters & $\begin{array}{c}\text { A - Female } \\
\text { (Other than FSW) } \\
\text { (Prompt) }\end{array}$ & $\begin{array}{c}\text { B - Female sex worker } \\
\text { (Prompt) }\end{array}$ & $\begin{array}{c}\text { C - Male sex worker } \\
\text { (Prompt) }\end{array}$ & $\begin{array}{c}\text { D - Male } \\
\text { (Other than MSW) } \\
\text { (Prompt) }\end{array}$ & $\begin{array}{l}\text { E - Hijra } \\
\text { (Prompt) }\end{array}$ \\
\hline \multirow[t]{3}{*}{ E12. } & \multirow[t]{3}{*}{$\begin{array}{l}\text { How often do you use a } \\
\text { condom when you have } \\
\text { sex with [ ] in last } 12 \\
\text { months? }\end{array}$} & 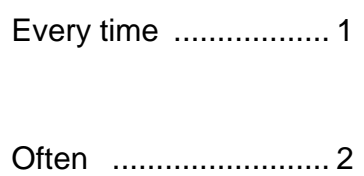 & Every time $\ldots \ldots \ldots \ldots \ldots \ldots . . . . . .1$ & Often $\ldots \ldots \ldots \ldots \ldots \ldots \ldots \ldots \ldots \ldots$ & Every time $\ldots \ldots \ldots \ldots \ldots \ldots \ldots$ & Often $\ldots \ldots \ldots \ldots \ldots \ldots \ldots \ldots \ldots \ldots$ \\
\hline & & Sometimes .................. 3 & Sometimes .................3 & Sometimes …............... 3 & Sometimes …................. & Sometimes $\ldots \ldots \ldots \ldots \ldots . . . . . . .3$ \\
\hline & & Never ............................ 4 & 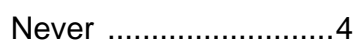 & Never ............................ 4 & 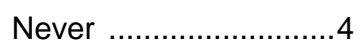 & 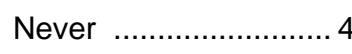 \\
\hline
\end{tabular}

If respondent say 'Never' in 'E12' Go to 'E15'

\begin{tabular}{|c|c|c|c|c|c|c|}
\hline E13 & $\begin{array}{l}\text { Did you use a condom } \\
\text { when you had sex with } \\
\text { [ ] the last time? }\end{array}$ & Yes $\ldots \ldots \ldots \ldots \ldots \ldots \ldots \ldots \ldots \ldots$ & 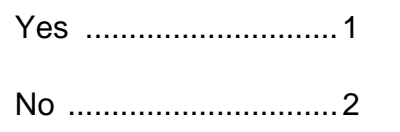 & Yes $\ldots \ldots \ldots \ldots \ldots \ldots \ldots \ldots \ldots \ldots$ & 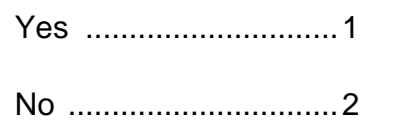 & Yes \\
\hline \multirow[t]{5}{*}{ E14. } & $\begin{array}{l}\text { Why did you use a } \\
\text { condom? }\end{array}$ & Avoid pregnancy .............1 & Avoid pregnancy ............. 1 & Avoid pregnancy .............1 & Avoid pregnancy .............. 1 & Avoid pregnancy ......... 1 \\
\hline & & Avoid giving a disease $\ldots . .2$ & Avoid giving a disease $\ldots . .2$ & Avoid giving a disease $\ldots 2$ & Avoid giving a disease $\ldots . .2$ & Avoid giving a disease 2 \\
\hline & & Avoid getting a disease ...3 & Avoid getting a disease... 3 & Avoid getting a disease. .3 & Avoid getting a disease $\ldots 3$ & Avoid getting a disease 3 \\
\hline & responses & Partner's wish...................... 4 & Partner's wish ................ 4 & Partner's wish................ 4 & Partner's wish ................... 4 & Partner's wish.............. 4 \\
\hline & & Others [specify] $\ldots \ldots \ldots \ldots \ldots . . .7$ & Others [specify] $\ldots \ldots \ldots \ldots \ldots \ldots$ & Others [specify] $\ldots \ldots \ldots \ldots \ldots . . .7$ & Others [specify] $\ldots \ldots \ldots \ldots \ldots \ldots$ & Others [specify] $\ldots \ldots \ldots \ldots . .7$ \\
\hline
\end{tabular}




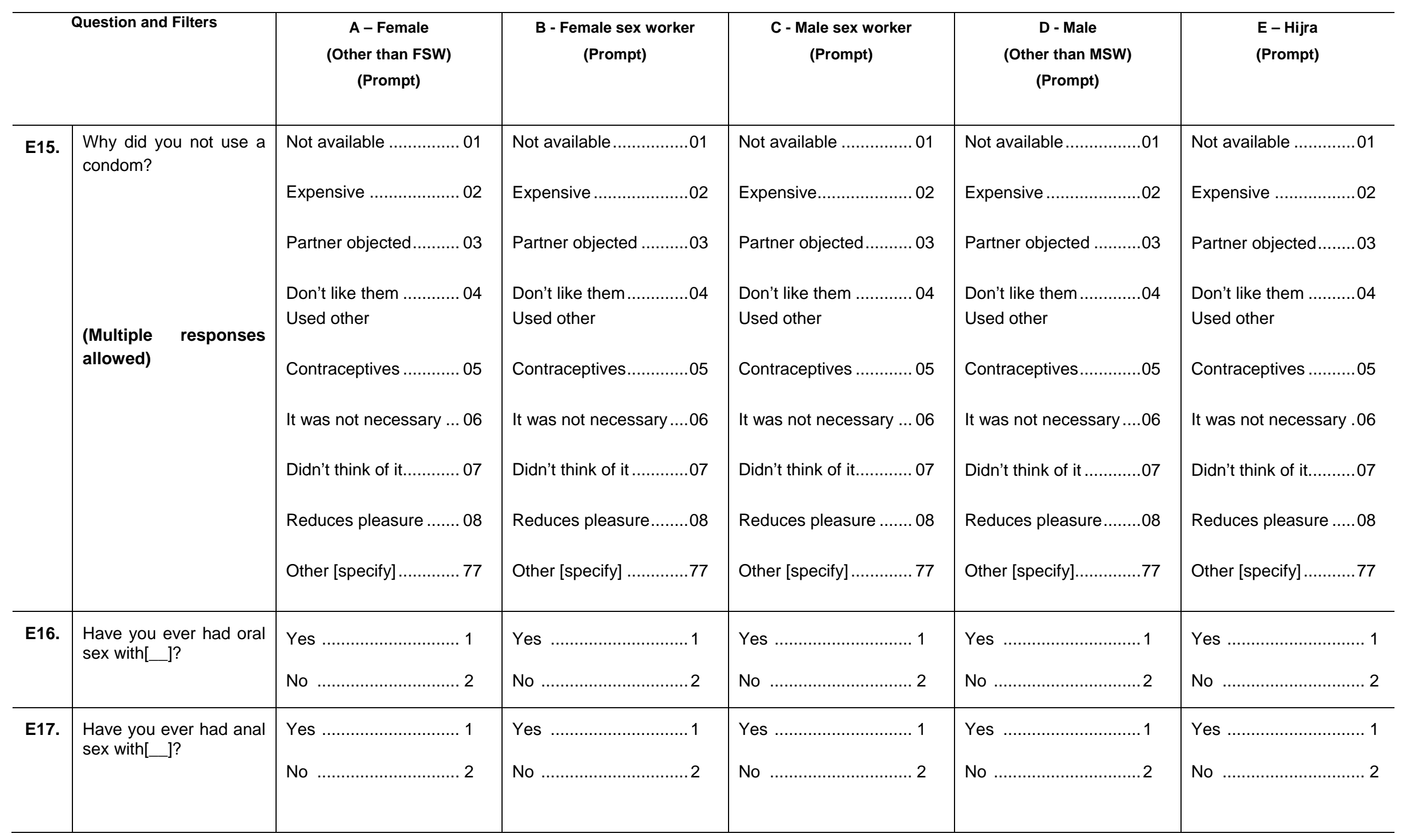


Now I am going to ask you a few questions that are related to your wife also.

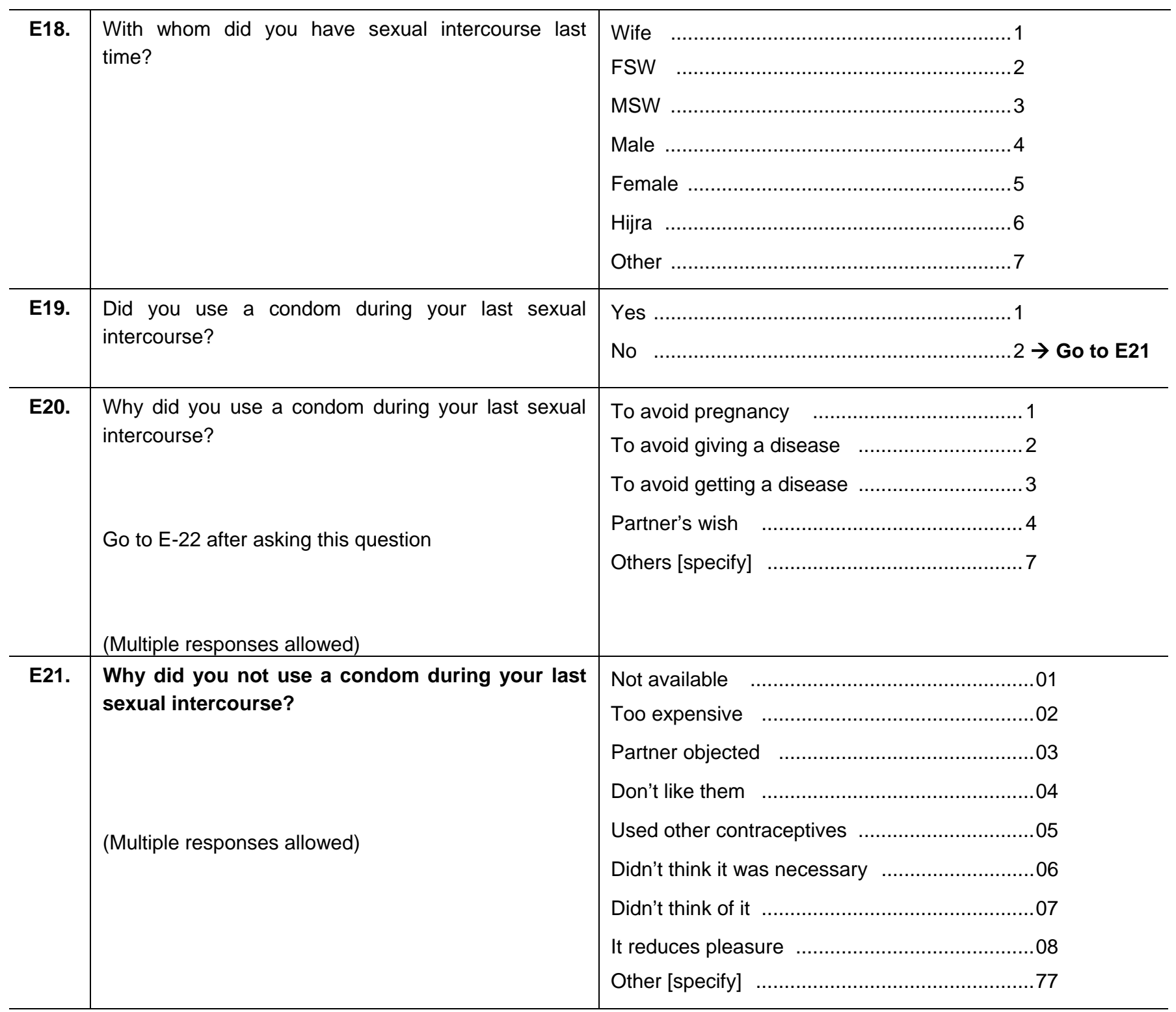

Now I would like to ask some questions about personal hygiene

\begin{tabular}{|c|c|c|}
\hline E22 & At what age were you circumcised? & 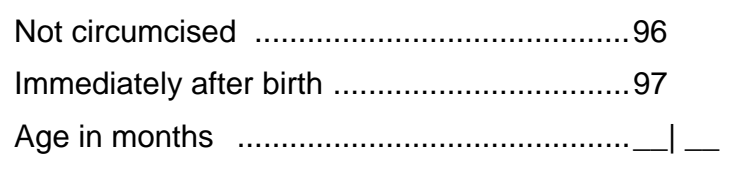 \\
\hline E23. & $\begin{array}{l}\text { How often do you wash the genital area after } \\
\text { urination? }\end{array}$ & 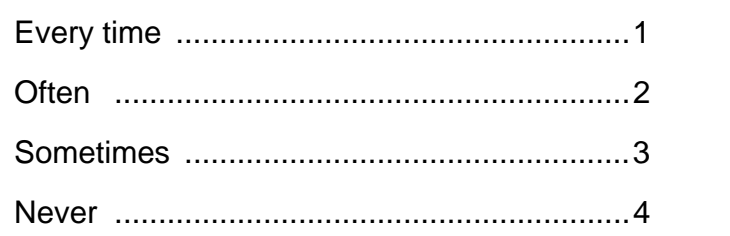 \\
\hline
\end{tabular}




\begin{tabular}{|c|c|c|}
\hline E24. & Do you wash your genital area before having sex? & 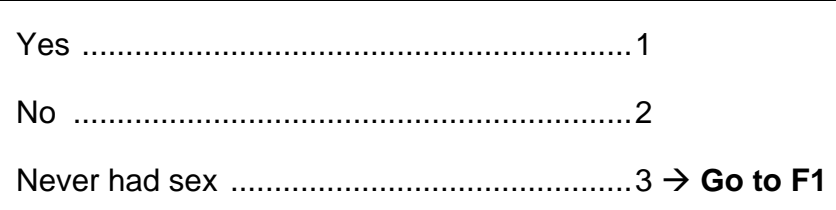 \\
\hline E25. & Do you wash your genital area after having sex? & Yes \\
\hline E26. & $\begin{array}{l}\text { Why do you wash your genital area after having sex? } \\
\text { (Multiple responses allowed) }\end{array}$ & $\begin{array}{l}\text { To avoid a disease } \\
\text { For personal hygiene } \\
\text { Religious injunction }\end{array}$ \\
\hline E27. & Do you pass urine soon after having sex? & Yes \\
\hline E28. & $\begin{array}{l}\text { Has there ever been a time during the last six } \\
\text { months when you wanted to have sex with your } \\
\text { wife but she did not? }\end{array}$ & $\begin{array}{l}\text { Yes } \\
\text { No } \\
\text { Unmarried }\end{array}$ \\
\hline E29. & $\begin{array}{l}\text { What did you do the last time you wanted to have sex } \\
\text { with your wife but she did not? }\end{array}$ & $\begin{array}{l}\text { Had sex after convincing her.................. } 1 \rightarrow \text { Go to F1 } \\
\text { Forced her to have sex } \\
\text { Did not have sex } \\
\text { Had sex with someone else }\end{array}$ \\
\hline E30. & $\begin{array}{l}\text { With whom did you have sex last time when you } \\
\text { wanted to have sex with your wife but she did not? }\end{array}$ & FCSW \\
\hline
\end{tabular}


Now Section - F: Blood Transfusion, Injection Taking And Drug Use History

\begin{tabular}{|c|c|c|}
\hline \multicolumn{2}{|c|}{ Questions and Filters } & \multirow{2}{*}{ 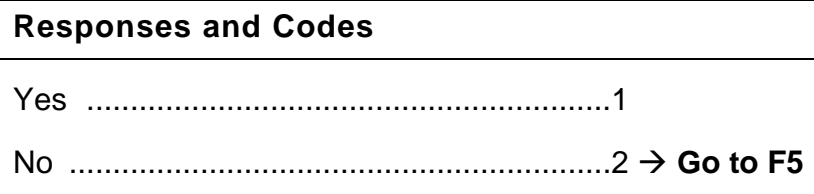 } \\
\hline F1. & Have you ever received any injection? & \\
\hline F2. & $\begin{array}{l}\text { How many times have you received injections in } \\
\text { last one year? }\end{array}$ & 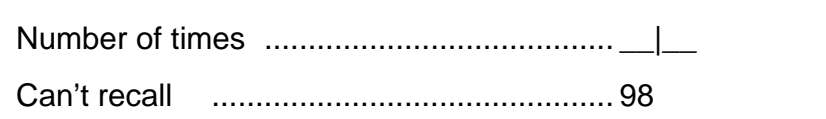 \\
\hline F3. & How long ago did you receive the last injection? & 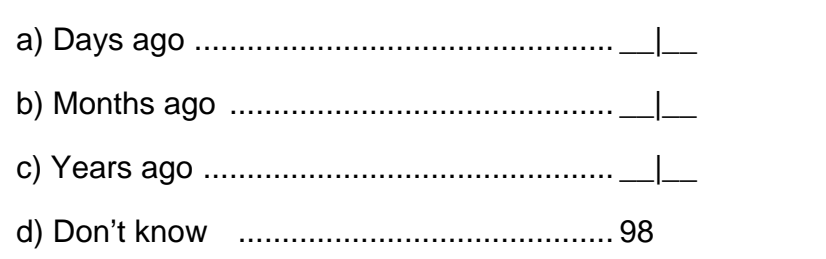 \\
\hline F4. & $\begin{array}{l}\text { Was the syringe/needle that was used the last time } \\
\text { new and unpacked or not opened in front of you? }\end{array}$ & 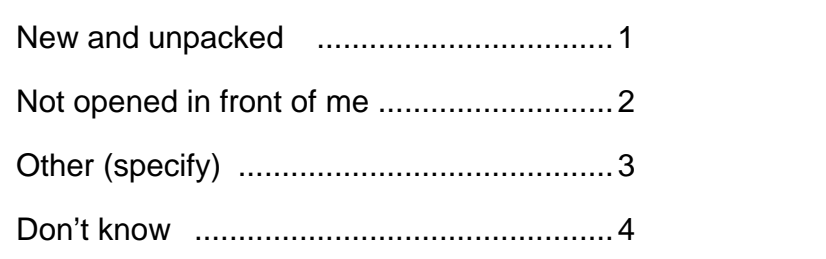 \\
\hline F5. & $\begin{array}{l}\text { Have you ever received a blood transfusion in your } \\
\text { lifetime? }\end{array}$ & Yes \\
\hline F6. & Did you make any payment for receiving blood? & 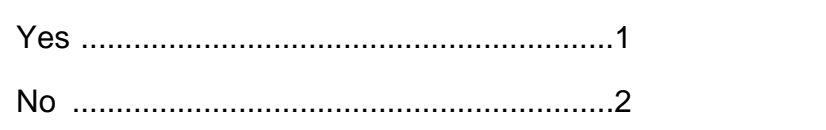 \\
\hline F7. & $\begin{array}{l}\text { How many times have you received a blood } \\
\text { transfusion? }\end{array}$ & 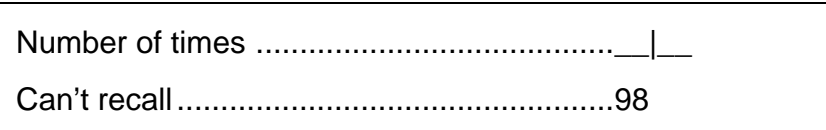 \\
\hline F8. & $\begin{array}{l}\text { The last time you received a blood transfusion did } \\
\text { you know it was screened for any disease? }\end{array}$ & 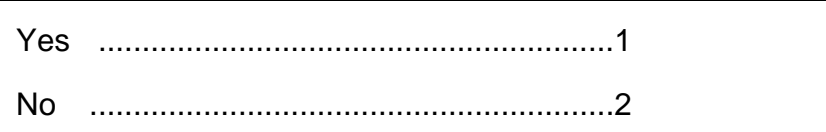 \\
\hline F9. & Have you ever donated blood? & 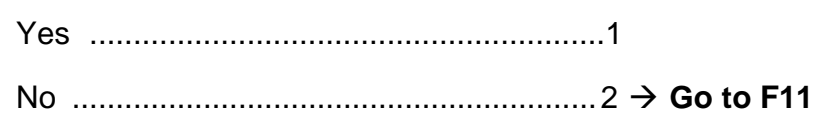 \\
\hline F10. & Did you ever rexceive payment for donating blood? & Yes \\
\hline F11. & Have you ever had your tooth extracted? & Yes \\
\hline F12. & How long ago did you have your tooth extracted? & 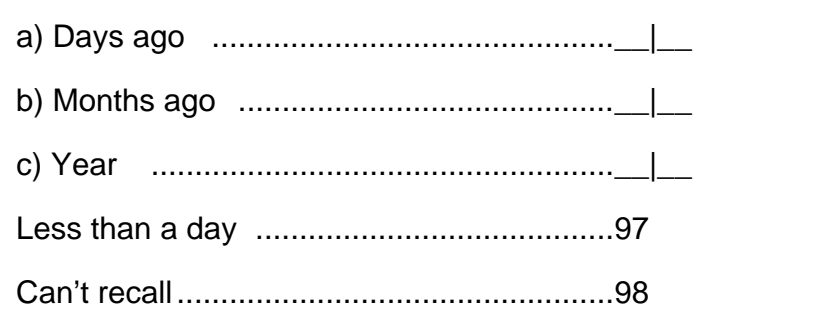 \\
\hline
\end{tabular}




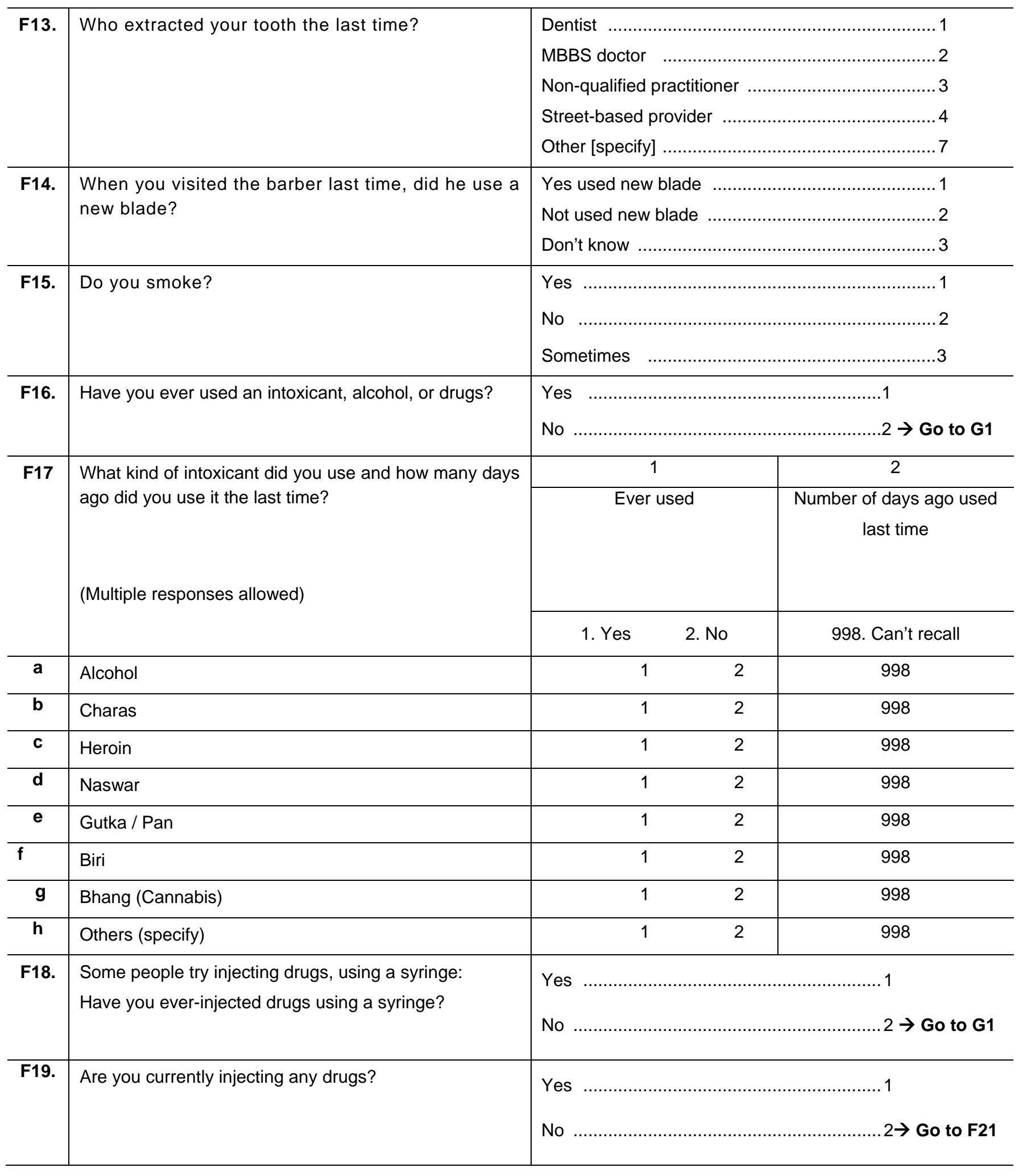




\begin{tabular}{|c|c|c|}
\hline F20. & $\begin{array}{l}\text { Which drug do you inject? } \\
\text { (Multiple responses allowed) }\end{array}$ & Tamigesic \\
\hline F21. & How frequently do you inject drugs? & $\begin{array}{l}\text { a) Number of time a day } \\
\text { b) Number of time a week } \\
\text { c) Number of time a month } \\
\text { d) Number of time a year }\end{array}$ \\
\hline F22. & $\begin{array}{l}\text { Last time you injected drugs did you use a new syringe } \\
\text { or shared the syringe? }\end{array}$ & 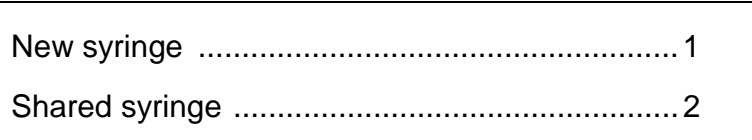 \\
\hline F23. & Last time where did you obtain the syringe? & Medical store \\
\hline F24 & $\begin{array}{l}\text { When injecting a drug in the last one month how often } \\
\text { did you use a needle or syringe that had been } \\
\text { unopened? }\end{array}$ & $\begin{array}{l}\text { Every time } \\
\text { Often } \\
\text { Sometimes } \\
\text { Never } \\
\text { Didn't inject last month }\end{array}$ \\
\hline
\end{tabular}




\section{Section - G: Knowledge about STls and Health Seeking Behavior}

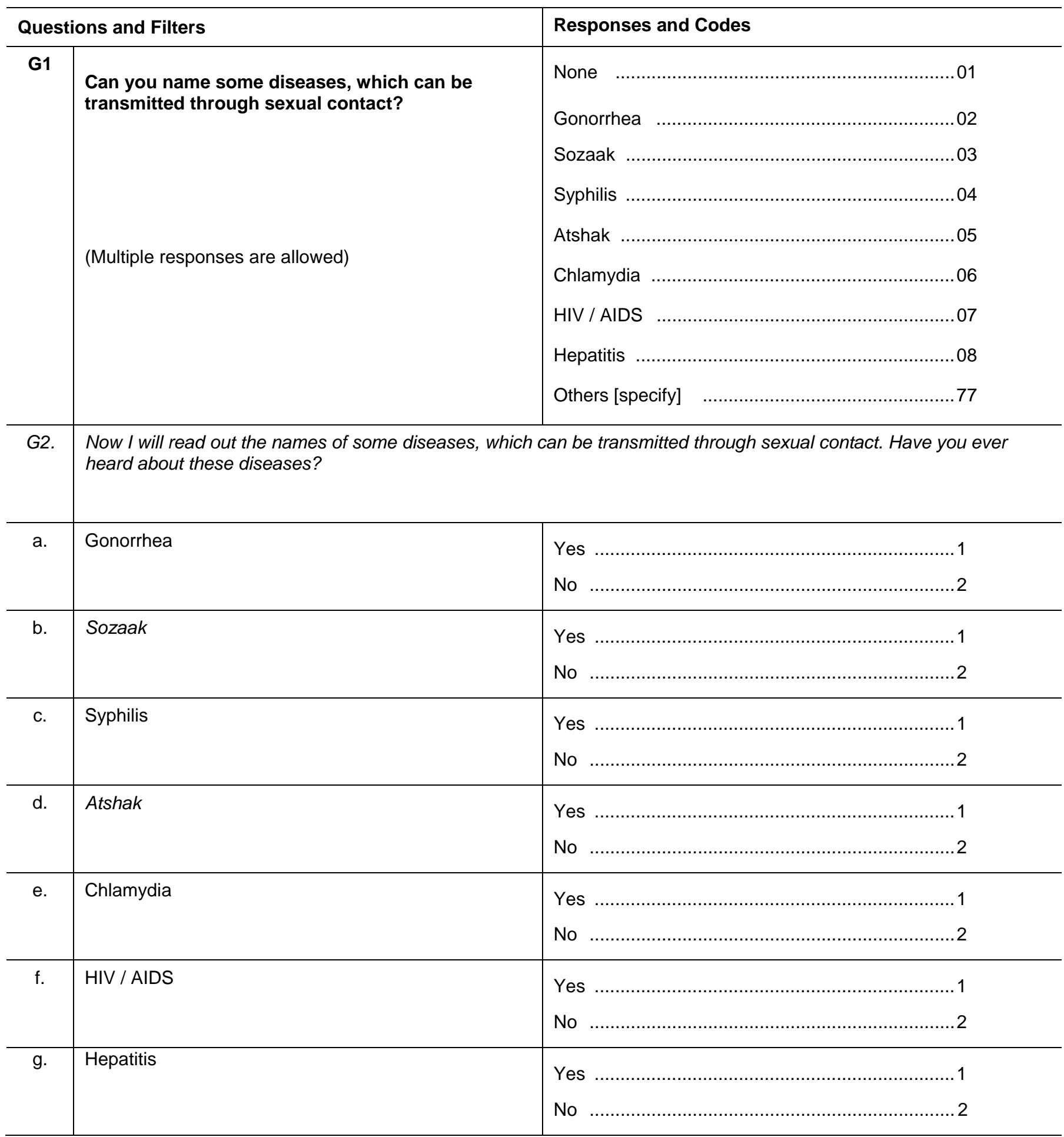




\begin{tabular}{|c|c|c|c|c|c|}
\hline G3. & $\begin{array}{l}\text { How can a person protect himself from sexually } \\
\text { transmitted diseases/infections? }\end{array}$ & $\begin{array}{l}\text { Yes } \\
\text { unprompted }\end{array}$ & Yes prompted & No & $\begin{array}{l}\text { Don't } \\
\text { know }\end{array}$ \\
\hline a. & Abstain from sex & 1 & 2 & 3 & 4 \\
\hline b. & Always use condoms & 1 & 2 & 3 & 4 \\
\hline c. & Have only one sex partner & 1 & 2 & 3 & 4 \\
\hline d. & Avoid sex workers & 1 & 2 & 3 & 4 \\
\hline e. & Other & 1 & 2 & 3 & 4 \\
\hline G4. & $\begin{array}{l}\text { In your opinion, what problems/complication can } \\
\text { occur if sexually transmitted diseases/infections } \\
\text { go untreated? } \\
\text { (Multiple responses allowed) }\end{array}$ & \multicolumn{4}{|c|}{ Infertility } \\
\hline G5. & $\begin{array}{l}\text { Where do men in your area usually prefer to go } \\
\text { for the treatment of sexually transmitted } \\
\text { diseases? } \\
\text { (Multiple responses allowed) }\end{array}$ & \multicolumn{4}{|c|}{$\begin{array}{l}\text { MBBS Doctor } \\
\text { Compounder/ Dispenser/ nurse } \\
\text { Hakeem } \\
\text { Quack } \\
\text { Homeopath }\end{array}$} \\
\hline G6. & $\begin{array}{l}\text { In your opinion, what are the reasons for } \\
\text { selection of this health care provider for treatment } \\
\text { of STIs? }\end{array}$ & \multicolumn{4}{|c|}{$\begin{array}{l}\text { Recommended by family } \\
\text { Recommended by friends } \\
\text { Physically accessible } \\
\text { Convenient timings } \\
\text { Qualified service provider } \\
\text { Economs privacy } \\
\text { Only facility available }\end{array}$} \\
\hline
\end{tabular}




\begin{tabular}{|c|c|c|c|}
\hline \multirow[t]{3}{*}{ G7 } & \multirow{2}{*}{$\begin{array}{l}\text { What are the main sources of information for men } \\
\text { about STIS? }\end{array}$} & 1 & \multirow{2}{*}{$\begin{array}{c}2 \\
\text { Rank of their } \\
\text { importance }\end{array}$} \\
\hline & & Sources of information & \\
\hline & $\begin{array}{l}\text { [Interviewer] Please rank the main sources of } \\
\text { information] } \\
\text { [Multiple responses allowed] }\end{array}$ & $\begin{array}{l}\text { a) Friends } \\
\text { b) Relatives } \\
\text { c) Newspapers } \\
\text { d) Radio/ TV } \\
\text { e) Literature } \\
\text { f) Doctor } \\
\text { g) Paramedical } \\
\text { i) Quacks } \\
\text { j) Other (specify) }\end{array}$ & $\begin{array}{ll}{[} & ] \\
{[} & ] \\
{[} & ] \\
{[} & ] \\
{[} & ] \\
{[} & ] \\
{[} & ] \\
{[} & ] \\
{[} & ]\end{array}$ \\
\hline G8. & $\begin{array}{l}\text { If someone in your area has a STI can he openly } \\
\text { discuss it with other people? }\end{array}$ & Yes & \\
\hline G9. & $\begin{array}{l}\text { If no, what are the reasons that he cannot openly } \\
\text { discuss STI problems with other people? } \\
\text { (Multiple responses allowed) }\end{array}$ & 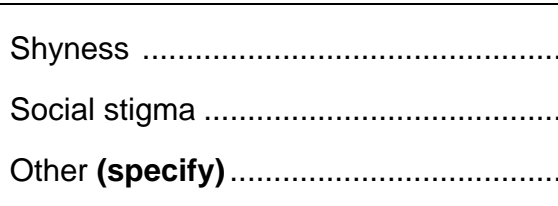 & \\
\hline G10. & $\begin{array}{l}\text { If you would have these STI problems with whom } \\
\text { would you discuss? } \\
\text { (Multiple responses allowed) }\end{array}$ & $\begin{array}{l}\text { No one } \\
\text { Wife } \\
\text { Father } \\
\text { Friend } \\
\text { Brother }\end{array}$ & \\
\hline G11. & $\begin{array}{l}\text { In your opinion what are the barriers in men for } \\
\text { seeking treatment for STIs? } \\
\text { (Multiple responses allowed) }\end{array}$ & $\begin{array}{l}\text { Denial in the culture } \\
\text { Shyness } \\
\text { Financial Barriers } \\
\text { Access Barriers } \\
\text { Non availability of facilities } \\
\text { Othe(specify) }\end{array}$ & $\begin{array}{r}\ldots 1 \\
\ldots 2 \\
\ldots 3 \\
\ldots 4 \\
\ldots 5 \\
\ldots 6 \\
\ldots 7\end{array}$ \\
\hline G12. & Have you ever heard about HIV or the word AIDS? & Yes &. $.2 \rightarrow$ Go to $\mathrm{G} 17$ \\
\hline
\end{tabular}




\begin{tabular}{|c|c|c|c|c|c|}
\hline G13. & $\begin{array}{l}\text { Can a healthy looking person be infected with the HIV } \\
\text { virus? }\end{array}$ & \multicolumn{4}{|c|}{ Yes No } \\
\hline G14. & How can HIV/AIDS be transmitted? & $\begin{array}{c}\text { Yes } \\
\text { unprompted }\end{array}$ & $\begin{array}{c}\text { Yes } \\
\text { Prompted }\end{array}$ & No & Don't know \\
\hline a. & Sexual contact & 1 & 2 & 3 & 4 \\
\hline b. & Transfusion of infected blood & 1 & 2 & 3 & 4 \\
\hline c. & Injections given by used syringe & 1 & 2 & 3 & 4 \\
\hline d. & From mother to infant through breastfeeding & 1 & 2 & 3 & 4 \\
\hline e. & From needle or sharp objects such as razors & 1 & 2 & 3 & 4 \\
\hline f. & Mosquito/insect bite & 1 & 2 & 3 & 4 \\
\hline g. & Sharing food with an infected person & 1 & 2 & 3 & 4 \\
\hline h. & Sharing clothes with an infected person & 1 & 2 & 3 & 4 \\
\hline i. & Sharing bedding with an infected person & 1 & 2 & 3 & 4 \\
\hline j. & By shaking hands with an infected person & 1 & 2 & 3 & 4 \\
\hline k. & Sharing toilet with an infected person & 1 & 2 & 3 & 4 \\
\hline I. & Other (specify) & 1 & 2 & 3 & 4 \\
\hline G15. & $\begin{array}{l}\text { How can a person protect himself or herself from } \\
\text { getting infected with HIV/AIDS? }\end{array}$ & $\begin{array}{c}\text { Yes } \\
\text { unprompted }\end{array}$ & $\begin{array}{c}\text { Yes } \\
\text { Prompted }\end{array}$ & No & Don't know \\
\hline a. & Abstain from sex & 1 & 2 & 3 & 4 \\
\hline b. & Use of a condom & 1 & 2 & 3 & 4 \\
\hline c. & Limit number of sex partners & 1 & 2 & 3 & 4 \\
\hline d. & Have only one sex partner & 1 & 2 & 3 & 4 \\
\hline e. & Avoid sex workers & 1 & 2 & 3 & 4 \\
\hline f. & Use sterilized needles & 1 & 2 & 3 & 4 \\
\hline g. & Require partner to take blood test & 1 & 2 & 3 & 4 \\
\hline h. & Staying away from patients & 1 & 2 & 3 & 4 \\
\hline i. & Other (specify) & 1 & 2 & 3 & 4 \\
\hline
\end{tabular}




\begin{tabular}{|c|c|c|}
\hline G16. & $\begin{array}{l}\text { From which sources did you receive information } \\
\text { regarding HIV/AIDS? } \\
\text { (Multiple responses allowed) }\end{array}$ & Doctors \\
\hline G17. & $\begin{array}{l}\text { Do you think that you are at risk of acquiring any } \\
\text { disease that can be transmitted through sexual } \\
\text { contact? }\end{array}$ & Yes No \\
\hline G18 & $\begin{array}{l}\text { Why do you think that you are not at risk of acquiring a } \\
\text { STI? } \\
\text { (Multiple responses allowed) }\end{array}$ & [Specify] \\
\hline G19 & $\begin{array}{l}\text { How would you rate the risk of your acquiring an STI? } \\
\text { (Prompt) }\end{array}$ & Low \\
\hline G20 & $\begin{array}{l}\text { Why do you think that you are at risk of acquiring a } \\
\text { STI? } \\
\text { (Multiple responses allowed) }\end{array}$ & [Specify] \\
\hline G21. & $\begin{array}{l}\text { How would you rate the risk of your acquiring } \\
\text { HIV/AIDs? }\end{array}$ & 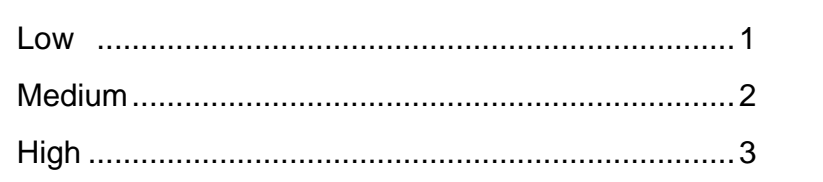 \\
\hline G22. & $\begin{array}{l}\text { Why do you think that you are at risk of acquiring } \\
\text { HIV/AIDs? } \\
\text { (Multiple responses allowed) }\end{array}$ & [Specify] \\
\hline G23. & $\begin{array}{l}\text { Why do you think that you are not at risk of acquiring } \\
\text { HIV/AIDs? } \\
\text { (Multiple responses allowed) }\end{array}$ & [Specify] \\
\hline
\end{tabular}




\begin{tabular}{|c|c|c|c|c|c|}
\hline G24. & $\begin{array}{l}\text { Do you know where people can go to get an HIV/AIDS } \\
\text { test done? }\end{array}$ & \multicolumn{4}{|c|}{ Yes $\mathrm{N}$} \\
\hline G25. & Have you ever had your blood tested for HIV/AIDS? & \multicolumn{4}{|c|}{ 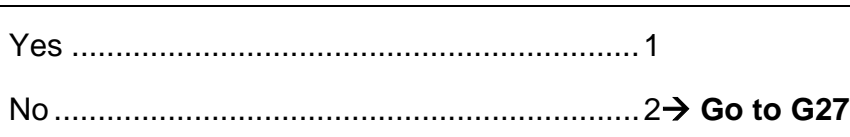 } \\
\hline G26. & Where did you get your blood tested for HIV/AIDS? & \multicolumn{4}{|c|}{ 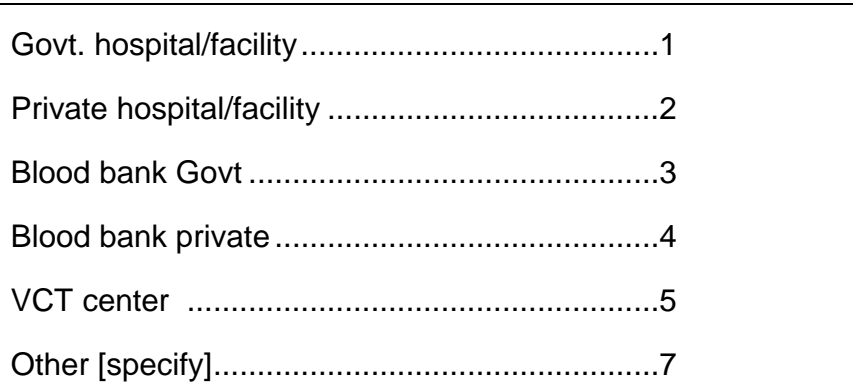 } \\
\hline G27. & Why did you have your blood tested for HIV/AIDS? & \multicolumn{4}{|c|}{ 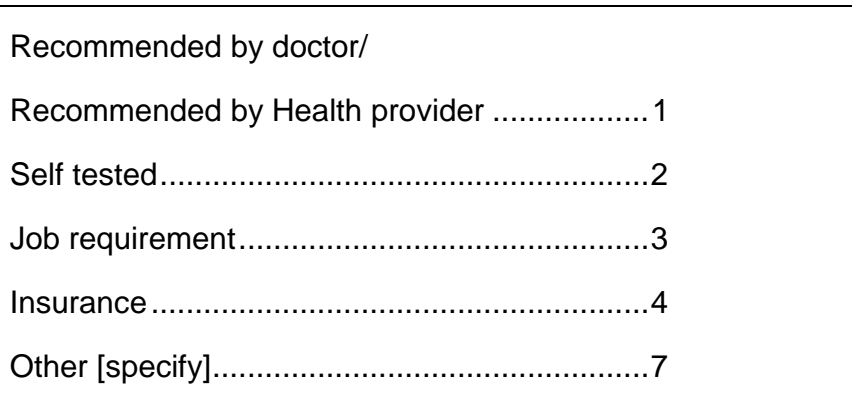 } \\
\hline G28. & $\begin{array}{l}\text { What measures have you adopted to protect yourself } \\
\text { from acquiring an STI including HIV? }\end{array}$ & $\begin{array}{l}\text { Yes } \\
\text { unprompted }\end{array}$ & $\begin{array}{c}\text { Yes } \\
\text { Prompted }\end{array}$ & No & Don't know \\
\hline a. & Abstain from sex & 1 & 2 & 3 & 4 \\
\hline b. & Use of a condom & 1 & 2 & 3 & 4 \\
\hline c. & Limit number of sex partners & 1 & 2 & 3 & 4 \\
\hline d. & Have only one sex partner & 1 & 2 & 3 & 4 \\
\hline e. & Avoid sex workers & 1 & 2 & 3 & 4 \\
\hline f. & Use sterilized needles & 1 & 2 & 3 & 4 \\
\hline g. & Require partner to take blood test & 1 & 2 & 3 & 4 \\
\hline h. & Staying away from patients & 1 & 2 & 3 & 4 \\
\hline G29. & $\begin{array}{l}\text { In your opinion, which is most dangerous route for } \\
\text { getting a STI-oral, anal, or vaginal sex? }\end{array}$ & 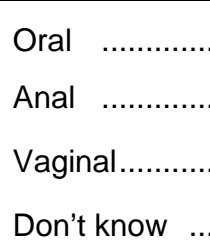 & , & ....... & $\begin{array}{r}\ldots \ldots .1 \\
\ldots \ldots . .2 \\
\ldots \ldots . .3 \\
\ldots \ldots . .4\end{array}$ \\
\hline
\end{tabular}




\begin{tabular}{|c|c|c|}
\hline G30 & $\begin{array}{l}\text { Some people use condoms while having sex, do you } \\
\text { know for what purposes condoms are used? } \\
\text { (Multiple responses allowed) }\end{array}$ & $\begin{array}{l}\text { To avoid getting disease } \\
\text { To avoid giving disease } \\
\text { To avoid pregnancy } \\
\text { Don't know }\end{array}$ \\
\hline G31 & $\begin{array}{l}\text { Can you name the places from where condoms can be } \\
\text { obtained? }\end{array}$ & 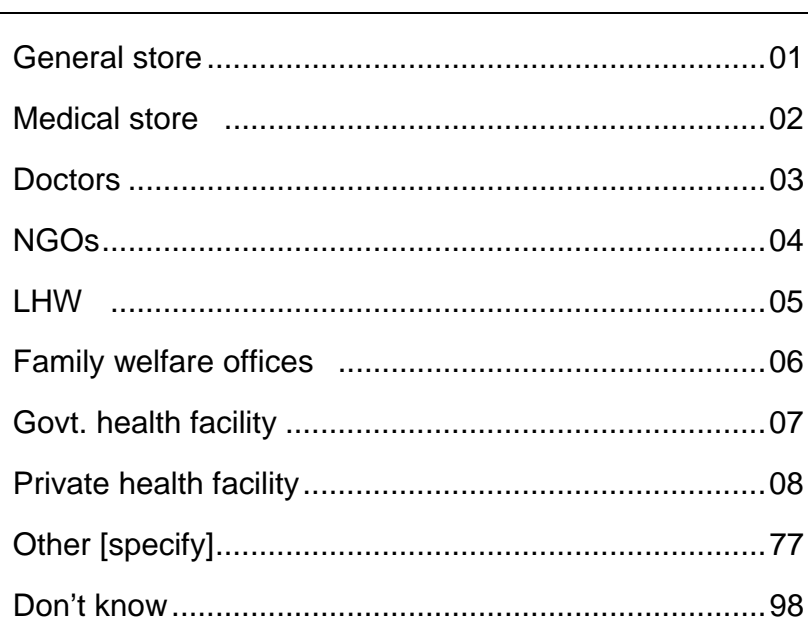 \\
\hline G32 & $\begin{array}{l}\text { Do you think it's difficult to find places to buy } \\
\text { condoms? }\end{array}$ & 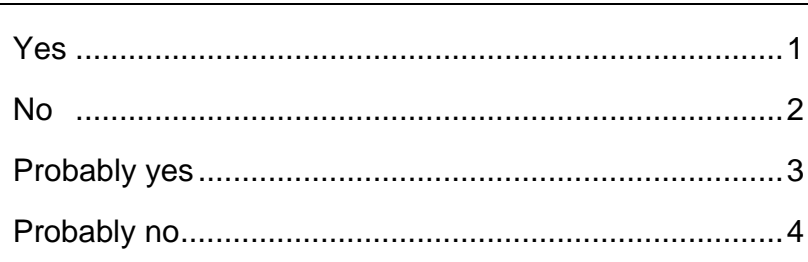 \\
\hline G33 & Have you ever used a condom? & Yes No \\
\hline
\end{tabular}


Section -H: Personal Experience with STIs

\section{[Interviewer] Please ask $\mathrm{H} 1$ of this section by row wise and all other questions of this column wise.}

\begin{tabular}{|c|c|c|c|c|}
\hline \multicolumn{2}{|c|}{ Questions and Filters } & \multicolumn{3}{|c|}{ Responses and Codes } \\
\hline & & Ulcer/sores in the genital area & Purulent discharge from penis & Pain during urination \\
\hline H1 & $\begin{array}{l}\text { During the last six months } \\
\text { have you } \\
\text { experienced.......? }\end{array}$ & 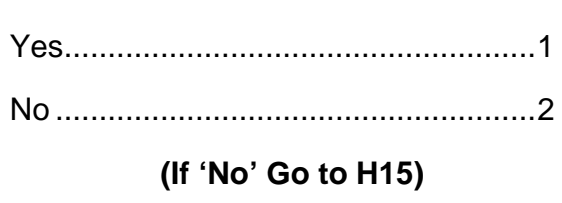 & Yes $\quad$ (If ‘No' Go to H15) & Yes (If 'No' Go to H15) \\
\hline H2. & $\begin{array}{l}\text { How long ago did you first } \\
\text { notice [ ]? }\end{array}$ & 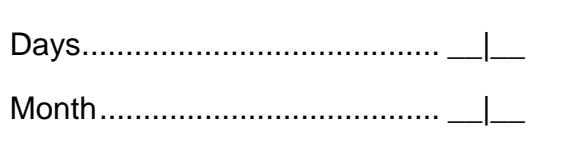 & 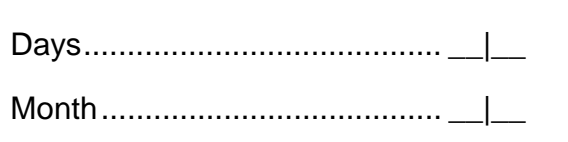 & 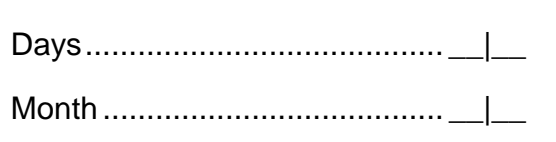 \\
\hline H3. & How long did [ ] last? & 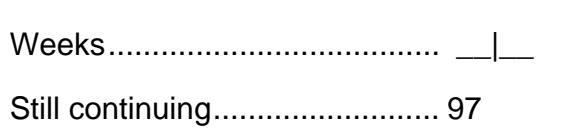 & 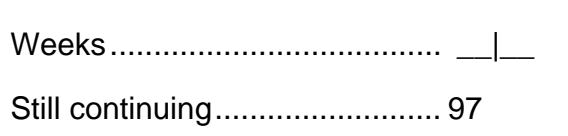 & 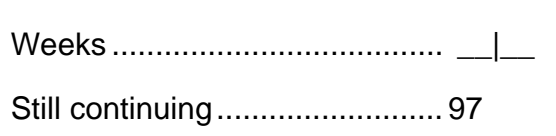 \\
\hline H4. & $\begin{array}{l}\text { With whom did you discuss [ } \\
\text { ] the problem? } \\
\text { (Multiple responses } \\
\text { allowed) }\end{array}$ & 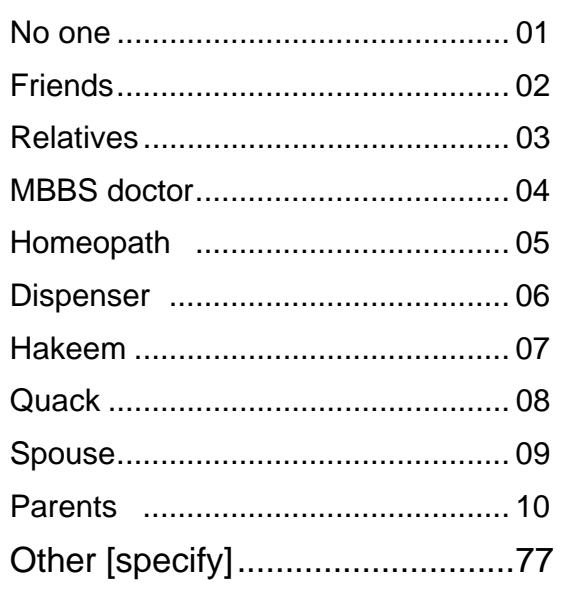 & 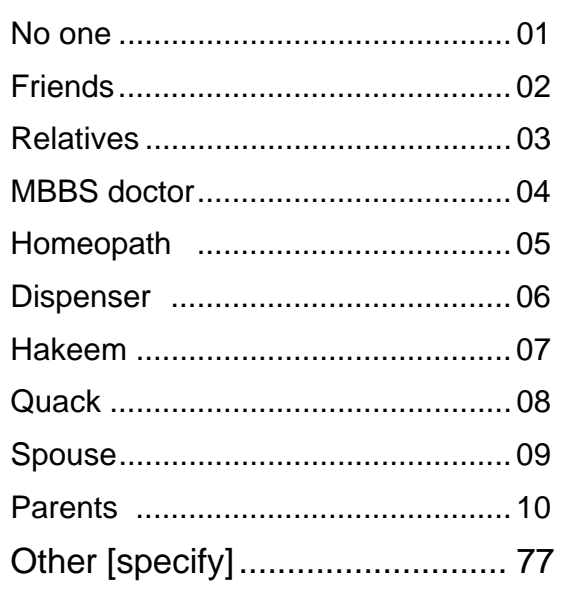 & $\begin{array}{l}\text { No one } \\
\text { Friends } \ldots \ldots \ldots \ldots \ldots \\
\text { Relatives }\end{array}$ \\
\hline H5. & $\begin{array}{l}\text { Did you seek treatment for [ } \\
\text { ] }\end{array}$ & No Y........................................... 2 & No & 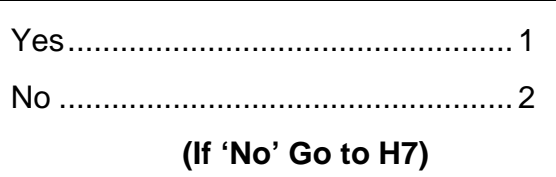 \\
\hline
\end{tabular}


[Interviewer] Please ask $\mathrm{H} 1$ of this section by row wise and all other questions of this column wise.

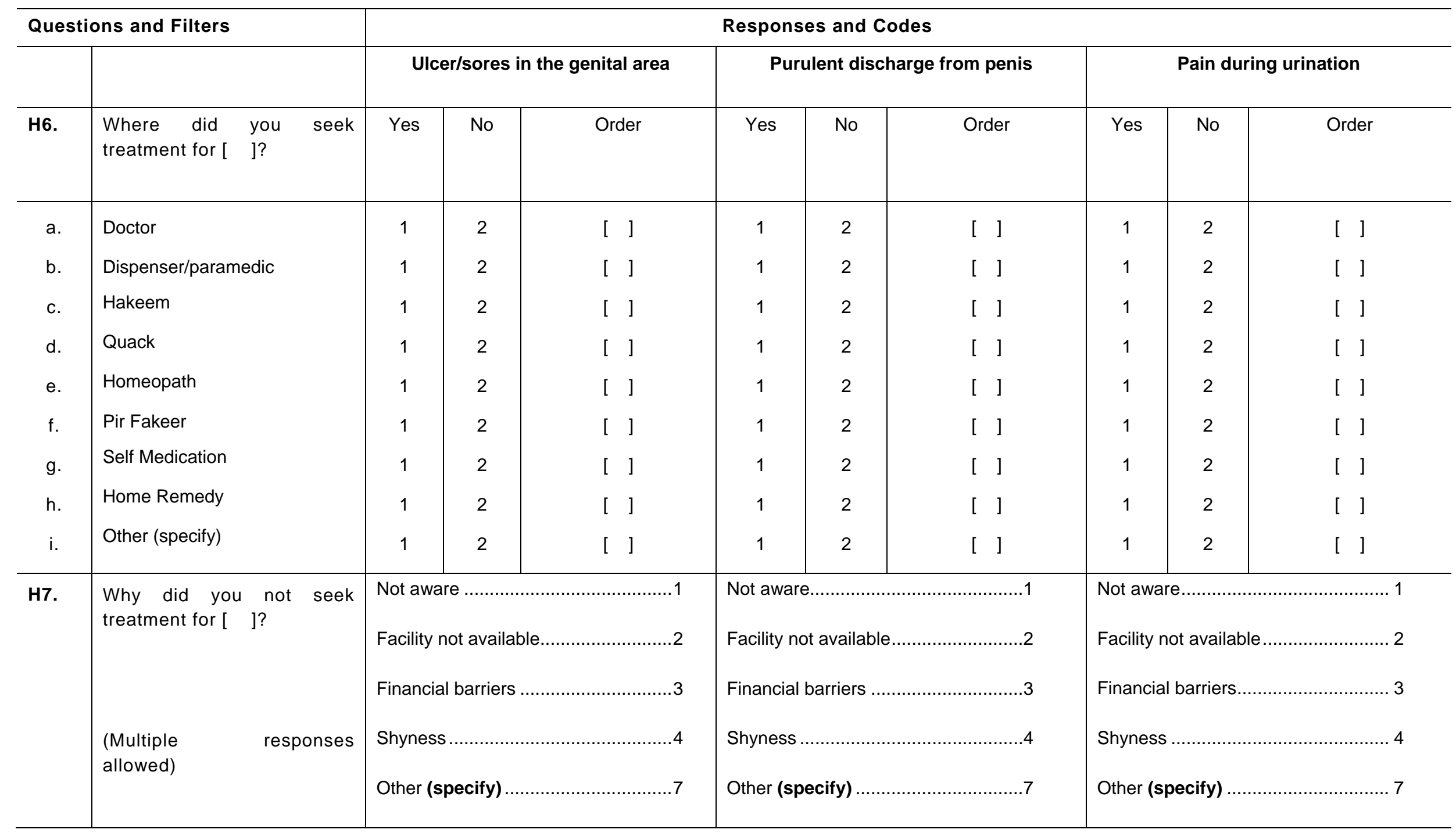


Interviewer check if sought treatment from more than one service provider then ask this question

Otherwise Go to $\mathrm{H} 10$

\begin{tabular}{|c|c|c|c|c|}
\hline \multicolumn{5}{|c|}{ 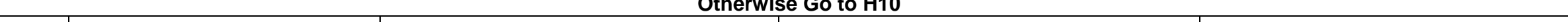 } \\
\hline & & Ulcer/sores in the genital area & Purulent discharge from penis & Pain during urination \\
\hline H8. & $\begin{array}{l}\text { Why did you seek treatment } \\
\text { with first [1] service provider } \\
\text { for [ ]? } \\
\text { (Multiple responses } \\
\text { allowed) }\end{array}$ & 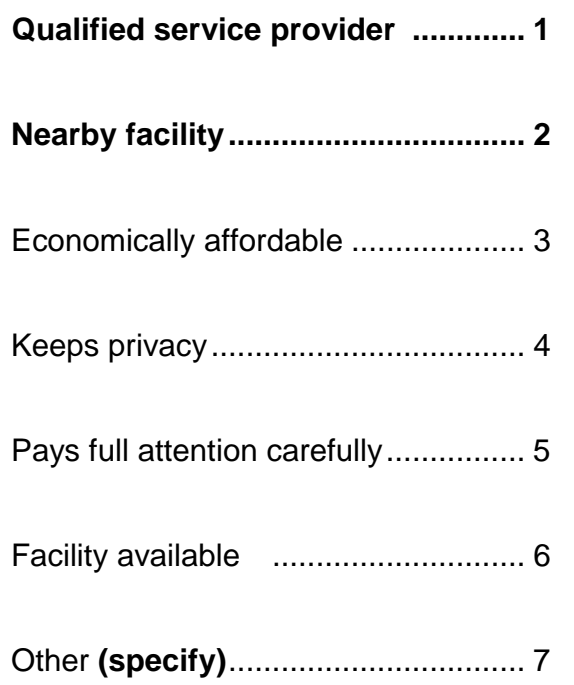 & 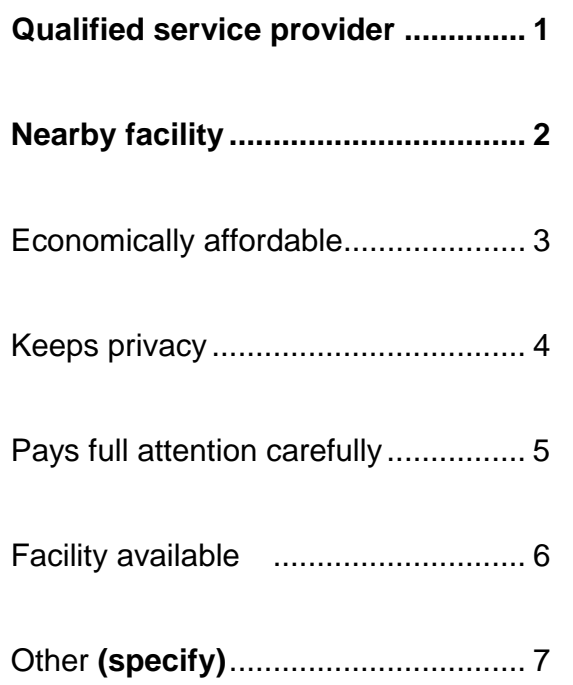 & 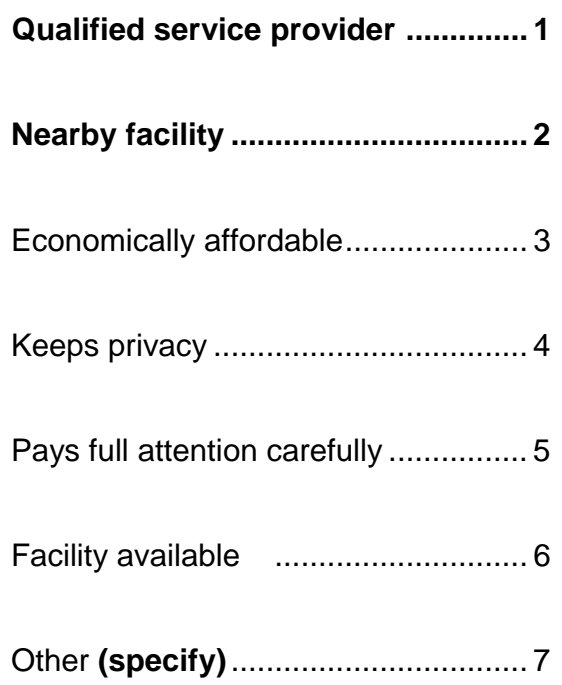 \\
\hline H9. & $\begin{array}{l}\text { Why did you seek treatment } \\
\text { with second [2] service } \\
\text { provider for }[\quad] \text { ? } \\
\\
\text { (Multiple responses } \\
\text { allowed) }\end{array}$ & $\begin{array}{l}\text { Not satisfied with previous one ......... } 1 \\
\text { Previous was unaffordable ................. } \\
\text { Previous was not qualified ................ } 3 \\
\text { Previous was not friendly ................. } 4 \\
\text { Previous don't explain problems well. } .5 \\
\text { Other (specify) ..................................7 }\end{array}$ & $\begin{array}{l}\text { Not satisfied with previous one .......... } 1 \\
\text { Previous was unaffordable ................. } \\
\text { Previous was not qualified ................ } \\
\text { Previous was not friendly ................. } 4 \\
\text { Previous don't explain problems well .5 } \\
\text { Treatment didn't work ........................ } 6 \\
\text { Other (specify) ...................................7 }\end{array}$ & $\begin{array}{l}\text { Not satisfied with previous one .......... } 1 \\
\text { Previous was unaffordable ................. } \\
\text { Previous was not qualified .............. } 3 \\
\text { Previous was not friendly ................. } \\
\text { Previous don't explain problems well .5 } \\
\text { Treatment didn't work........................6 } \\
\text { Other (specify) ................................... }\end{array}$ \\
\hline
\end{tabular}




\begin{tabular}{|c|c|c|c|c|}
\hline & & Ulcer/sores in the genital area & Purulent discharge from penis & Pain during urination \\
\hline H10. & $\begin{array}{l}\text { What was the problem } \\
\text { diagnosed as? } \\
\text { (Multiple responses } \\
\text { allowed) }\end{array}$ & 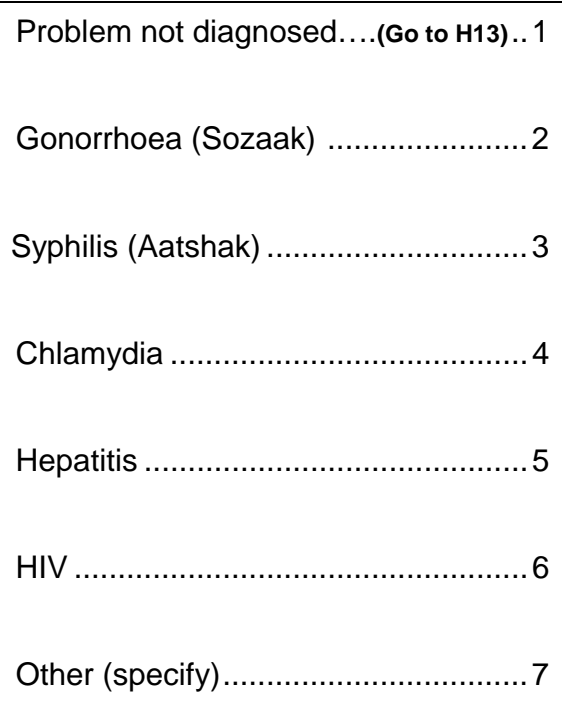 & 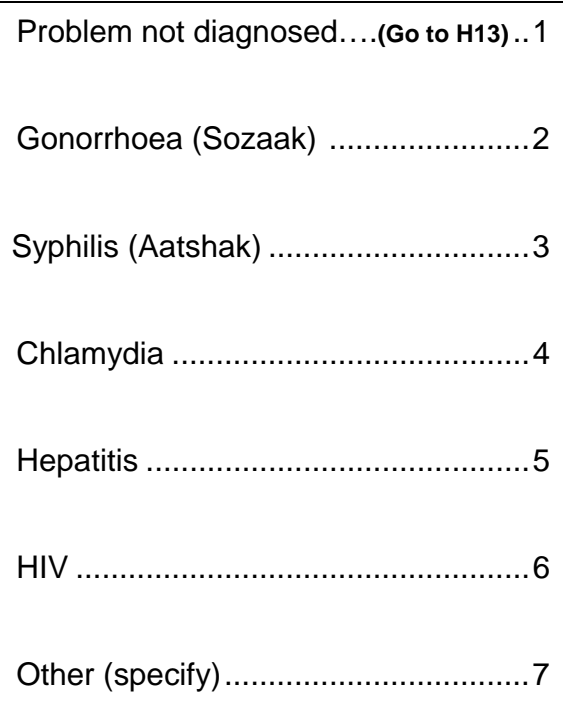 & 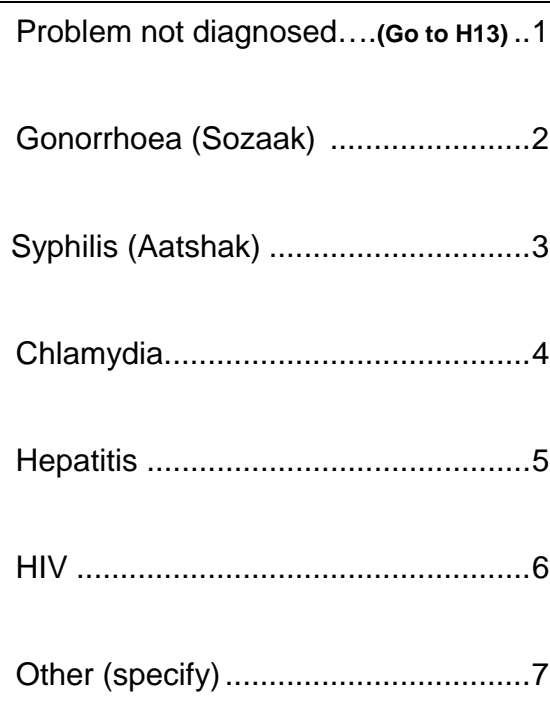 \\
\hline H11. & $\begin{array}{l}\text { How was the problem } \\
\text { diagnosed? }\end{array}$ & $\begin{array}{l}\text { History/sign symptoms ..................... } 1 \\
\text { Lab test ...................................... } 2 \\
\text { Other (specify) }\end{array}$ & 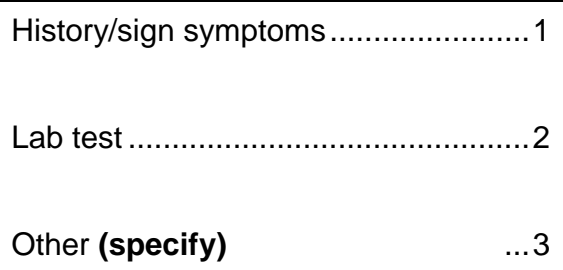 & $\begin{array}{l}\text { History/sign symptoms } \ldots \ldots \ldots \ldots \ldots \ldots \ldots 1 \\
\text { Lab test ..................................................... } \\
\text { Other (specify) }\end{array}$ \\
\hline H12. & $\begin{array}{l}\text { How much money have you } \\
\text { spent on the problem } \\
\text { diagnosed including its } \\
\text { treatment? }\end{array}$ & 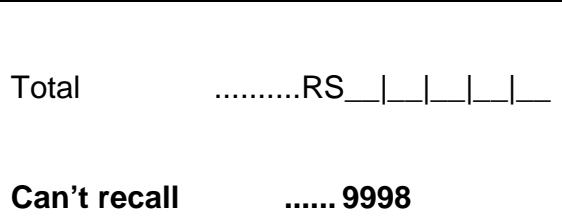 & 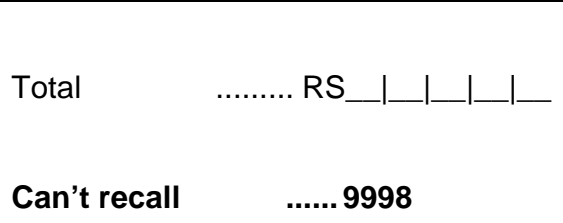 & 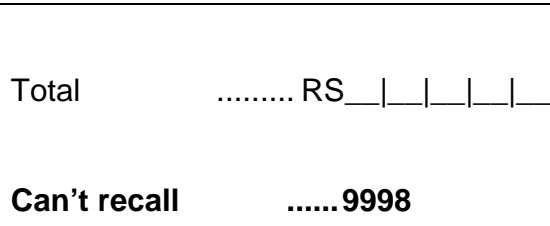 \\
\hline
\end{tabular}




\begin{tabular}{|c|c|c|c|c|}
\hline & & Ulcer/sores in the genital area & Purulent discharge from penis & Pain during urination \\
\hline H13. & $\begin{array}{l}\text { What preventive measure } \\
\text { did you use to prevent your } \\
\text { partner from getting this } \\
\text { same disease? } \\
\text { (Multiple responses } \\
\text { allowed) }\end{array}$ & Avoid intercourse & Avoid intercourse & Avoid intercourse \\
\hline H14. & $\begin{array}{l}\text { Was your partner aware } \\
\text { about your disease? }\end{array}$ & 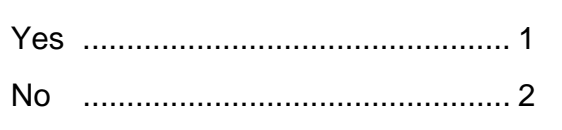 & $\begin{array}{l}\text { Yes } \\
\text { No }\end{array}$ & $\begin{array}{l}\text { Yes } \\
\text { No }\end{array}$ \\
\hline
\end{tabular}




\begin{tabular}{|c|c|c|}
\hline H15. & $\begin{array}{l}\text { Do you know of any facility in your area that provides } \\
\text { services for the treatment of STIs? }\end{array}$ & Yes No \\
\hline H16. & $\begin{array}{l}\text { Can you tell us the name of that facility? } \\
\text { (Could be more than one response) }\end{array}$ & Name: \\
\hline H17. & $\begin{array}{l}\text { Have you visited a health facility in the last } 6 \text { months } \\
\text { to obtain STI information or services? }\end{array}$ & Yes \\
\hline
\end{tabular}




\section{Section - I: Sexual Behavior and Attitude}

\begin{tabular}{|c|c|c|}
\hline \multicolumn{2}{|c|}{ Questions and Filters } & \multirow[b]{2}{*}{$\begin{array}{l}\text { Responses and Codes } \\
\text { Strongly agree } \\
\text { Agree }\end{array}$} \\
\hline $\mathrm{l}-1$. & $\begin{array}{l}\text { If a man has a chance to establish sexual relations } \\
\text { before marriage, in your opinion should he avail this } \\
\text { opportunity? }\end{array}$ & \\
\hline $\mathrm{I}-2$ & $\begin{array}{l}\text { If a married man has a chance to establish sexual relations } \\
\text { outside of marriage, in your opinion should he avail this } \\
\text { opportunity? }\end{array}$ & Strongly agree \\
\hline $\mathrm{I}-3$. & $\begin{array}{l}\text { If a man has a chance to establish sexual relations with a } \\
\text { man, in your opinion should he avail this opportunity? }\end{array}$ & Strongly agree \\
\hline $\mathrm{I}-4$. & $\begin{array}{l}\text { In your opinion, what are the risks of having sex with a } \\
\text { man? } \\
\text { (Multiple responses allowed) }\end{array}$ & Specify \\
\hline $\mathrm{I}-5$. & $\begin{array}{l}\text { In your opinion, what are the risks of having sex with a } \\
\text { female other than your wife? } \\
\text { (Multiple responses allowed) }\end{array}$ & Specify \\
\hline $\mathrm{I}-6$. & $\begin{array}{l}\text { In your opinion, what are the risks of having sex with a } \\
\text { hijra? } \\
\text { (Multiple responses allowed) }\end{array}$ & Specify \\
\hline
\end{tabular}


I-7. $\quad$ Can you name a few places that are commonly associated with sexual activities?

For interviewer: Allow respondent to name places associated with sexual activity. Once he is finished, tell him: "Thank you. I will now mention some places, and I would like to ask you to tell me whether or not you think these places are associated with sexual activity."

\begin{tabular}{|c|c|c|c|c|c|}
\hline \multicolumn{2}{|r|}{ Places commonly associated with sexual activities } & \multirow{2}{*}{$\begin{array}{c}\begin{array}{c}\text { Yes, } \\
\text { unprompted }\end{array} \\
1\end{array}$} & \multirow{2}{*}{$\begin{array}{c}\begin{array}{c}\text { Yes, } \\
\text { prompted }\end{array} \\
2\end{array}$} & \multirow{2}{*}{$\begin{array}{l}\text { No } \\
3\end{array}$} & \multirow{2}{*}{$\begin{array}{c}\begin{array}{c}\text { Don't } \\
\text { know }\end{array} \\
4\end{array}$} \\
\hline a. & Mini cinema homes & & & & \\
\hline b. & Brothels & 1 & 2 & 3 & 4 \\
\hline c. & Hotels & 1 & 2 & 3 & 4 \\
\hline d. & Hotels near truck stand & 1 & 2 & 3 & 4 \\
\hline e. & Hotels near bus stand & 1 & 2 & 3 & 4 \\
\hline f. & Hotels near railway station & 1 & 2 & 3 & 4 \\
\hline g. & Internet cafes & 1 & 2 & 3 & 4 \\
\hline h. & Video shops & 1 & 2 & 3 & 4 \\
\hline i. & Snooker club & 1 & 2 & 3 & 4 \\
\hline j. & Other (specify) & 1 & 2 & 3 & 4 \\
\hline I-8. & Have you heard about mini cinema? & 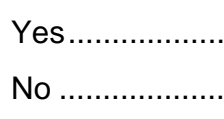 & $\ldots$ & & to to 110 \\
\hline I-9. & Have you ever gone to mini cinema? & 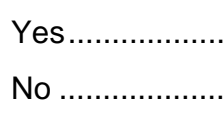 & $\ldots$ & ..... & \\
\hline I-10. & Do you have any access to the internet? & 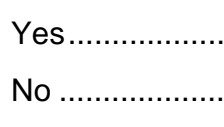 & . & ....... & \\
\hline I-11. & Have you ever seen pornographic materials? & 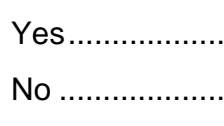 & . & … & io to 113 \\
\hline $\mathrm{I}-12$ & $\begin{array}{l}\text { What kind of pornographic material have you ever } \\
\text { seen? } \\
\text { (Multiple responses allowed) }\end{array}$ & $\begin{array}{l}\text { Printed materia } \\
\text { Videos/CDs ...... } \\
\text { Playing cards... } \\
\text { Internet............ } \\
\text { Others (specify }\end{array}$ & 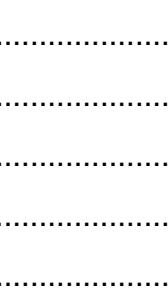 & .......... & \\
\hline I-13 & $\begin{array}{l}\text { Have any type of media in any way influenced your } \\
\text { sexual behavior? }\end{array}$ & $\begin{array}{l}\text { Yes } \\
\text { No }\end{array}$ & $\ldots$ & $\cdots$ & to to $\mathrm{I15}$ \\
\hline
\end{tabular}




\begin{tabular}{|c|c|c|c|}
\hline \multirow[t]{3}{*}{ I-14 } & \multirow{2}{*}{$\begin{array}{l}\text { Which medium of communication has in the most } \\
\text { influenced your sexual behavior? }\end{array}$} & 1 & 2 \\
\hline & & Type of media & Order \\
\hline & (Multiple responses allowed) & b) Cable/dish & $\begin{array}{ll}{[} & ] \\
{[} & ] \\
{[} & ] \\
{[} & ] \\
{[} & ] \\
{[} & ] \\
{[} & ] \\
{[} & ] \\
{[} & ] \\
{[} & ]\end{array}$ \\
\hline I-15 & $\begin{array}{l}\text { In your opinion, what kinds of health programs are } \\
\text { needed for men regarding their sexual health } \\
\text { problems? }\end{array}$ & & \\
\hline I-16 & Interview ended at (write in 24 hours) & -1_L_ & \\
\hline
\end{tabular}

We may be returning here to conduct an interview with you again. We will be interested in finding out your experiences over time. Please be assured that the information you have provided will only be used for research at the Population Council.

Will it be all right if we come back to talk to you again after some time?

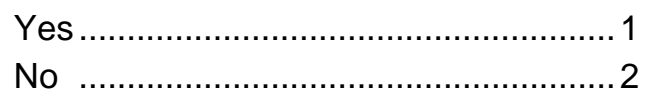

Thank you very much for answering these questions. The interview is completed. Do you have any questions for me? 